Nilgün Dağlar-Sezer

Stiftungen,

Zivilgesellschaft und

soziale Differenz

Eine qualitative Studie

zu gesellschaftspolitischen

Stiftungsprojekten 
Nilgün Dağlar-Sezer

Stiftungen, Zivilgesellschaft und soziale

Differenz 
Nilgün Dağlar-Sezer

\section{Stiftungen, \\ Zivilgesellschaft und soziale Differenz}

Eine qualitative Studie zu gesellschaftspolitischen Stiftungsprojekten

Budrich UniPress Ltd.

Opladen • Berlin • Toronto 2019 
Bibliografische Information der Deutschen Nationalbibliothek

Die Deutsche Nationalbibliothek verzeichnet diese Publikation in der Deutschen

Nationalbibliografie; detaillierte bibliografische Daten sind im Internet über

http://dnb.d-nb.de abrufbar.

(C) 2019 Dieses Werk ist beim Verlag Budrich UniPress erschienen und steht unter der Creative Commons Lizenz Attribution-ShareAlike 4.0 International (CC BY-SA 4.0): https://creativecommons.org/licenses/by-sa/4.0/

Diese Lizenz erlaubt die Verbreitung, Speicherung, Vervielfältigung und Bearbeitung bei Verwendung der gleichen CC-BY-SA 4.0-Lizenz und unter Angabe der UrheberInnen, Rechte, Änderungen und verwendeten Lizenz.

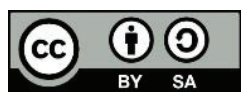

Dieses Buch steht im Open-Access-Bereich der Verlagsseite zum kostenlosen Download bereit (https://doi.org/10.3224/86388813).

Eine kostenpflichtige Druckversion kann über den Verlag bezogen werden. Die Seitenzahlen in der Druck- und Onlineversion sind identisch.

$\begin{array}{ll}\text { ISBN } & 978-3-86388-813-8 \\ \text { eISBN } & 978-3-86388-454-3 \\ \text { DOI } & 10.3224 / 86388813\end{array}$

Typographisches Lektorat und Satz: Anja Borkam, Jena Umschlaggestaltung: Bettina Lehfeldt, Kleinmachnow - www.lehfeldtgraphic.de 


\section{Danksagung}

„Auch ein langer Weg beginnt mit dem ersten Schritt“ lautet ein Sprichwort. Als 2009 die ersten Überlegungen für ein Promotionsvorhaben im Raum standen, war nicht ganz klar, wie lange der Weg sein würde: Die Fragestellung erwies sich thematisch als zweigeteilt, auch auf verschiedene Disziplinen. Doch auch ein langer Weg ist endlich. Dass diese Dissertation und damit dieses Buch ein Ende finden konnte, liegt zum einen an meinem Doktorvater Prof. Dr. Michael Meuser. Er schaffte die Strukturen und nahm das Thema inhaltlich an. Jeder seiner Hinweise war Goldwert. Damit einher geht der Dank an die Fakultät 12 der TU Dortmund, wo die Promotion absolviert wurde.

Zum anderen konnte das Buch nur mit Hilfe von Kamuran Sezer fertig gestellt werden, der fachlich und in Form des futureorg instituts strukturell unterstützend in allen Phasen zur Verfügung stand.

PD Dr. Matthias Freise danke ich für die unkomplizierte Übernahme des Zweitgutachtens, was keine Selbstverständlichkeit darstellt. JProf. Karolina Barglowski hat dankenswerterweise bei der Promotionsprüfung mitgewirkt.

Mit Jana Blum (Transkriptionen), Martin Rath (Endlektorat) und Şerife Gözübek (Graphikdesign) verlief die Zusammenarbeit besonders angenehm und verbindlich. Ein großer Dank gilt Benjamin Neumann für die kritische Durchsicht von Teilen des Manuskripts. Ebenso danke ich Sarah Hampel und Paula Wiesemann für wichtige Impulse im Abschlussteil sowie Dr. André Christian Wolf dafür, das Thema in seinen Anfängen mitentwickelt zu haben. An dieser Stelle gilt mein Dank auch Frau Prof. Dr. Annette Zimmer, die mir ebenfalls in der frühen Phase der Dissertation unterstützend zur Seite stand.

Ohne das Vertrauen der Interviewten aus den Stiftungsorganisationen wäre keine Erhebung möglich gewesen. Leider kann ich sie nicht namentlich auflisten, möchte aber an dieser Stelle ausdrücklich darauf hinweisen.

Meiner Familie, insbesondere meinen Eltern Ayşe und Kadir Dağlar, danke ich für das Interesse und die Zuversicht. Für den Übergang zur endgültigen Niederschrift war die Anwesenheit meiner Mutter unverzichtbar.

Familie Sezer hat abseits der beruflichen Arena die Strukturen aufrechterhalten, die alles möglich gemacht haben. Ihnen und allen, die mitgefiebert haben, gilt meine Verbundenheit.

Doch das alles führt erst zum Ziel, wenn im Zeitmanagement die richtigen Prioritäten gesetzt werden. Wie diese aussehen können, hat mich Gökhan 
Kasım Sezer gelehrt. Bei ihm und Kamuran Sezer möchte ich mich abschließend von ganzem Herzen bedanken. 
Dies ist so, weil das Denken die Ordnung unter den Dingen erfasst, ganz gleich, ob sie auf natürliche Weise oder durch (menschliche) Übereinkunft geschehen. Wenn es also beabsichtigt ist, eine Sache hervorzubringen, dann ist es wegen der Ordnung unter den Geschehnissen unerlässlich, deren Grund, Ursachen oder Bedingungen zu begreifen, denn diese sind in den meisten Fällen die Grundprinzipien dieser Sache, da sie nur in deren Folge entsteht.

(Aus: Ibn Khaldūn: Die Muqaddima. Betrachtungen zur Weltgeschichte, 1377 [2011]: 405) 



\section{Inhaltsverzeichnis}

Abbildungs- und Tabellenverzeichnis ............................................................. 11

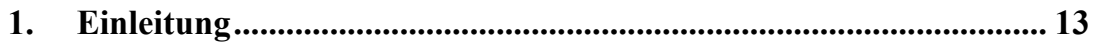

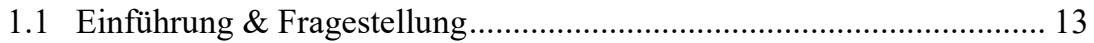

1.2 Theoretische \& methodische Rahmung …......................................... 16

2. Stiftungen im Spannungsfeld von Staat und Gesellschaft ................ 22

2.1 Standortbestimmungen im gesellschaftlichen Kontext ....................... 22

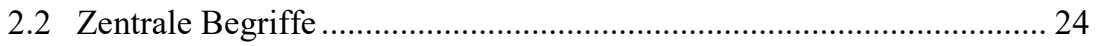

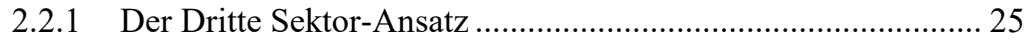

2.2.2 Das Konzept der Zivilgesellschaft .......................................... 27

2.2.3 Quo vadis Zivilgesellschaftsforschung .................................. 30

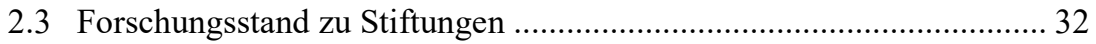

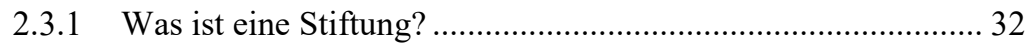

2.3.2 Stiftungslandschaft in Deutschland.......................................... 35

2.3.3 Stand der Stiftungsforschung................................................. 40

2.4 Stiftungen als Organisationen der Zivilgesellschaft........................... 43

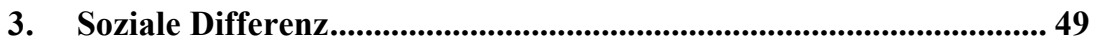

3.1 Herausforderungen einer Konzeption soziale Differenz ..................... 49

3.1.1 Ausgangssituation................................................................ 49

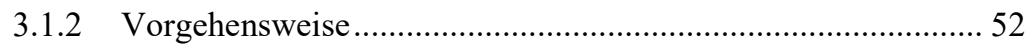

3.2 Sozialkonstruktivistische Ansätze sozialer Differenz ........................ 57

3.2.1 Sozialkonstruktivistische Grundlagen ................................... 57

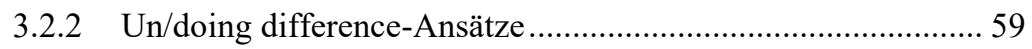

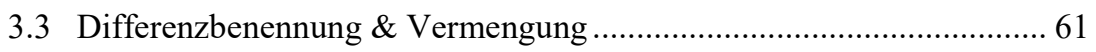

3.3.1 Kulturwissenschaftliche Grenzziehungskonzepte...................... 61

3.3.2 Forschungsansätze als Kritik am Bestehenden ......................... 64

3.3.3 Ungleichheitstheoretischer Ansatz Intersektionalität................ 67

3.3.4 Anwendungsorientierter Ansatz: Vielfalt/Diversity ................ 70

3.4 Analyserahmen für soziale Differenz................................................ 73 


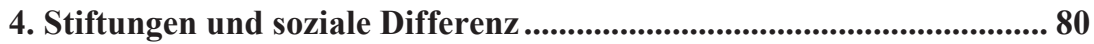

4.1 Methodisches Design für die Auswertung ............................................ 80

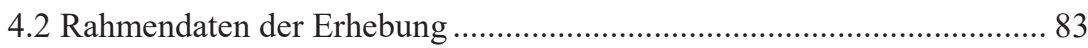

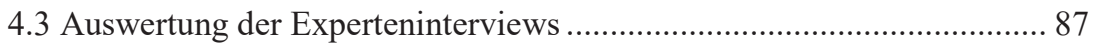

4.3.1 Die Interviewsituationen und die Fälle ....................................... 87

4.3.2 Tätigkeitsprofil der interviewten Personen ................................... 88

4.3.3 Eigendarstellungen im Akteurshandeln..................................... 92

4.3.4 Stiftungen zwischen Staat und Gesellschaft.............................. 104

4.3.5 Beziehungsherstellung und Selbstverortung ............................ 118

4.3.6 Argumentationskonstellationen hinsichtlich sozialer

Differenzkategorien .......................................................... 128

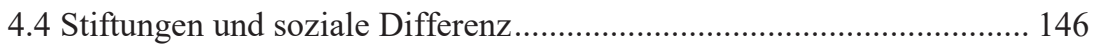

4.4.1 Sozio-ökonomischer Status ...................................................... 146

4.4.2 Migration/Ethnizität/Religion ................................................. 148

4.4.3 Lebensabschnitt/Alter/Lebenslage ........................................... 149

4.4.4 Geschlecht ....................................................................... 150

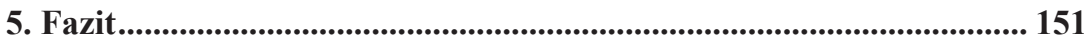

5.1 Rückblick: Standortbestimmung der vorliegenden Studie .................... 151

5.2 Ergebnis: Ambivalenz der Organisationsform Stiftung und

Vielschichtigkeit der gesellschaftlichen Wirkebene ........................... 155

5.3 Ausblick: Stiftungen als Akteure der öffentlichen Sphäre .................... 162

Quellenverzeichnis ............................................................................... 164 


\section{Abbildungs- und Tabellenverzeichnis}

Abbildung 1: Der Dritte Sektor als heuristisches Modell .......................... 26

Abbildung 2: Prozentuale Verteilung der gewichteten Hauptgruppen der Stiftungszwecke bei Stiftungserrichtungen in Dekaden seit 1951

Tabelle 1: Entstehungsgeschichte der sogenannten „verbände-“ bzw. „parteinahen“ Stiftungen in Deutschland auf Bundesebene bis 2017 38

Tabelle 2: $\quad$ Sortierung der relevanten Konzepte zu sozialer Differenz .. 75

Tabelle 3: Formale Position der Befragten und Zuständigkeiten nach eigenen Angaben

Tabelle 4: $\quad$ Konzeption soziale Differenz und Bezug zu empirischen Daten 158 



\section{Einleitung}

\subsection{Einführung \& Fragestellung}

„Die deutsche Demokratie ist mittlerweile stark genug, um auch mit Neonazis zu leben. Aber der türkische Gemüsehändler ist es nicht.“ Mit diesen Worten leitete Heribert Prantl, Chefredakteur der „Süddeutsche Zeitung“ am 6. Dezember 2012 seinen Kommentar zum NPD-Verbotsantrag ein. Indes wirkt die These ehrenwert: In Anspielung an die NSU-Mordserie wird für den Fortbestand der demokratischen Grundordnung der Minderheitenschutz prominent platziert - wozu eben der türkische Gemüsehändler zu gehören scheint. Die Gesamtgesellschaft wird wie ein Gegenschaubild als starke Beschützerin daneben gestellt. Wenn soziale Gruppen benannt und in einen Kontext gebracht werden, eröffnen sich viele Analyseebenen. Wer wird als was bezeichnet und in Anspielung auf welche Geschehnisse wie skizziert? Welches gesellschaftliche Bild wird befördert?

Die Dekonstruktion von sozialer Welt gehört seit je her zu den Grunddisziplinen der Soziologie. Wie das Eingangszitat zeigt, beschäftigen sich Menschen nicht erst seit ein paar hundert Jahren mit der Frage, was die (soziale) Welt konstituiert und wie diese funktioniert - wenngleich zu unserer heutigen Zeit in Hinblick auf die naturalisierenden oder theologisierenden Argumentationen Abstriche zu machen sind. So bezeichnet Weidner (2017) die weitläufigen Ausführungen des eingangs zitierten andalusisch-arabischen Gelehrten Ibn Khaldūn als „durchaus im Weber'schen Sinn zu verstehende - Entzauberung der mittelalterlichen Weltvorstellungen des Islam." (Weidner 2017: 17) Weidner weist darauf hin, dass er zu Recht in der Forschung mit den später lebenden europäischen Philosophen wie Machiavelli, Montesquieu oder Vico verglichen wurde. (vgl. ebd.)

Thesen zur gesellschaftlichen Ordnung und die Einordnung sozialer Gruppen bildet ein vieldiskutiertes Feld. Gerade im Diskurs um Demokratie und Gemeinschaft spielen zivilgesellschaftliche Vereinigungen eine wichtige Rolle. In Form von freiwillig und unentgeltlich getätigter Arbeit für die Gemeinschaft werden sie als gesellschaftlicher Kitt skizziert (Zimmer 1996, 2007). Dabei fächern sich Organisationstypen, die sich im zivilgesellschaftlichen Rahmen verstehen, thematisch und handlungslogisch sehr weit auf. $\mathrm{Zu}$ diesen gehören Stiftungsorganisationen als besondere ihrer Art. Denn Stiftungen eröffnen als Mischung von politischem Akteur und gemeinwohlorientierter Organisation wichtige Handlungsspielräume für politische Gruppen. Die Tätigkeit der politischen Stiftungen bildet allerdings nur einen gut sichtbaren 
Ausschnitt, denn fast 22.800 Stiftungen ${ }^{1}$ gibt es in Deutschland, teils in Form Jahrhunderte alter Institutionen, insbesondere jedoch in einer steigenden Zahl von Neugründungen. Nicht nur Parteien, auch Unternehmen, Privatpersonen, Kommunen und Kirchen erweitern mit Stiftungen ihre gesellschaftliche Reichweite. Sie bündeln bestimmte Sichtweisen auf die Gesellschaft in einem organisationalen Gewand, initiieren oder finanzieren soziale Projekte und beratschlagen sich zu politischen Themen. Parteinahe Stiftungen erhalten jährlich Millionenbeträge von der öffentlichen Hand, die sie als Stiftung handlungsfähig halten (vgl. Klaassen 2016).

Wenngleich der Aktionsradius von Stiftungen weitläufig ist, sind sie als politische und soziale Akteure weitgehend unerforscht. Relativ einig ist sich die Literatur darin, dass Stiftungen in ihrer Handlungslogik nicht den ,typischen“ Sphären „Markt“ und „Staat" unterworfen sind (vgl. Adloff 2004: 280). Im Übrigen findet Stiftungsforschung, wenn überhaupt, aus drei Perspektiven statt: Die umfangreichste Literatur liegt im Bereich der quantitativen Deskription von Stiftungsdaten vor (Bundesverband Deutscher Stiftungen 2013a; Bundesverband Deutscher Stiftungen 2013b; Anheier 2003). Weitergehende Erkenntnisse bringen die Beiträge zu politischen Stiftungen (Heisterkamp 2014; Pascher 2001; Klaassen 2016; von Reden 2015), die diese in verschiedenen Spannungsfeldern untersuchen. Als dritter Punkt sind unter Einbezug der historischen Perspektive die Skizzen zu einer „Stiftungstheorie“ zu erwähnen (Campenhausen 2015; Strachwitz 2016; Strachwitz 2015b; Strachwitz 2007), denen die empirische Fundierung noch fehlt. Insgesamt sind in erster Linie qualitative Studien zu Stiftungen kaum vorhanden. Mit ihrer Hilfe ließen sich sehr viel besser Selbstverständnis und gesellschaftspolitische Einstellungen rekonstruieren. Noch 2002 bezeichnete Frank Adloff Stiftungen als ,black boxes" (Adloff 2002: 3), bei denen man nicht genau wisse, was in ihnen geschehe (vgl. ebd.). Vor allem in den letzten Jahren holt die Stiftungsforschung auf: So verknüpfen Anheier u. a. (2017a; 2017b; 2017c) die vorliegenden punktuellen Erkenntnisse aus Historie, Zahlenmaterial und Selbstauskunft der Stiftungen miteinander und reichern diese um eine internationale Perspektive an.

Vor diesem Hintergrund zeichnet sich ein Forschungsfeld ab, das trotz einiger vorliegender Erkenntnisse noch viele Lücken aufweist. Es beginnt mit der Schwierigkeit, das Forschungsfeld präzise benennen zu können, bereits bei der Bezeichnung „Stiftung“ oder „Stiftungswesen“ selbst. Der Begriff der Stiftung regelt nicht eindeutig, um was für eine Organisation es sich handelt, weder im juristischen noch im sozialwissenschaftlichen Sinne. Abgesehen von der personellen und monetären Verwobenheit mit der politischen Sphäre lohnt sich der Blick auf die konkrete Programmatik in Stiftungsprojekten: Denn durch das Festlegen von Projektzielen, durch die Feststellung von Bedarfen 
und bedürftigen Zielgruppen definieren Stiftungen soziale Gruppen mit, die sich von anderen Gruppen zu unterscheiden scheinen. Wie es überhaupt zu der jeweiligen Programmatik gekommen ist und mit welchen Absichten diese durchgeführt wird, kann hier eine interessante Fragestellung sein. Es lässt sich vermuten, dass von Stiftungen ausgehende Zielgruppendefinitionen sehr heterogen sind, was bereits ein kurzer Blick in ihre Broschüren und Internetseiten nahelegt. Es bedeutet, dass soziale Differenz durch die Programmatik von kollektiven Akteuren, in diesem Beispiel Stiftungen, erzeugt und verstetigt wird. Fraglich ist, auf welche Art und Weise sich dann Stiftungen beim Umgang mit unterschiedlichen Differenzmerkmalen hervortun. Ist eine Kombinatorik aus verschiedenen Zielgruppen (jung \& weiblich; alt \& migrantisch) nicht naheliegend? Diese Zielgruppenheterogenität lässt sich mit dem Begriff der sozialen Differenz zusammenfassen, die auf ihre Herstellungspraxis hin zu untersuchen wäre. Genauer formuliert stellt sich die Frage, wie in der operativen Arbeit von Stiftungen soziale Differenz hervorgebracht wird. Es soll also untersucht werden, wie die Stiftungen die Projekte initiieren und umsetzen und welches Selbstverständnis bezogen auf ihre gesellschaftliche Positionierung ermittelt werden kann. Aus ,stiftungstheoretischer' Sicht interessiert zudem die Frage, wie Stiftungen ihr gesellschaftliches Engagement vor dem Hintergrund eines vielfach diskutierten Demokratiedefizits von demokratisch nicht legitimierten Akteuren begründen und wie sie sich sodann gegenüber staatlichen Behörden und Institutionen positionieren. In Hinblick auf das Kernanliegen der Arbeit, das auf die Benennungspraxis sozialer Grenzziehungen abzielt, stellt sich weitergehend die Frage, welches Menschenbild Stiftungen angesichts heterogener Gesellschaftsstrukturen und sozialstrukturell ungleicher Teilhabechancen transportieren. Welche Intentionen erwähnen sie zudem aus einer auto-organisationalen Betrachtung heraus? Es ist davon auszugehen bzw. eine Annahme der Untersuchung, dass Stiftungen Themen auf unterschiedlichen Wegen ermitteln: aus öffentlichen Debatten, Themen aus wissenschaftlicher Forschung, aber auch subjektiv generierte Projektziele zum Beispiel aus der lokalen Perspektive oder der individuellen Positionierung als politischer Akteur. Es stellt sich folglich die Frage, wie sie diese Themensetzungen tatsächlich generieren, verwirklichen und die Wahl der Beteiligten begründen. Der empirische $\mathrm{Zu}$ gang erfolgt durch Daten aus Interviews mit Repräsentantinnen und Repräsentanten von Stiftungen, die aufgrund ihrer gehobenen Position in der Organisation einen Überblick über diese grundsätzlichen Fragen der Programmatik Auskunft geben können. Mit diesem Forschungsvorhaben ist es zwar nicht möglich, eine Art Wirkungsmessung durchzuführen. Gewinnbringend ist die vorliegende Studie jedoch dahingehend, dass Differenzforschung aus der Mesoperspektive eines zivilgesellschaftlichen Akteurs bisher kaum vorzufinden ist; so auch Sonja Kubisch (2008: 326). Einen besonderen inhaltlichen Impuls 
und die Erkenntnis, dass die Bearbeitung einer solchen Themenkonstellation realisierbar ist, liefert Kubischs Dissertation „Habituelle Konstruktion sozialer Differenz. Eine rekonstruktive Studie am Beispiel von Organisationen der freien Wohlfahrtspflege“ (2008). Im Rahmen ihrer Dissertation führte Kubisch Gruppendiskussionen durch. Mittels einer dokumentarischen Auswertung rekonstruierte sie Habitustypen von zivilgesellschaftlichen Organisationen der freien Wohlfahrtspflege mit Blick auf die Konstruktion sozialer Differenz und die Sinngebungsmuster, die sich in scheinbar unverfänglichen Narrationen zu sozialer Differenz verbergen.

Die vorliegende Untersuchung eröffnet nun den Blick für die Handhabung sozialer Differenz durch einen weiteren Nonprofit-Akteur. Diese Studie zu Stiftungen ist allerdings durch die Datenerhebung mittels leitfadengestützten Interviews methodisch anders angelegt, wie im Weiteren näher dargelegt wird.

Alles in allen bilden Stiftungen durch ihre spezifische Verfasstheit und mit den unterschiedlichen Zugängen ihres sozialen Engagements ein interessantes Forschungsfeld.

\subsection{Theoretische \& methodische Rahmung}

Die vorliegende Arbeit gliedert sich in die vier folgenden Kapitel: Zunächst wird der Frage nachgegangen, wie Stiftungen im Spannungsfeld von Staat und Gesellschaft zu verorten und wie sie als Akteure der Zivilgesellschaft zu bewerten sind (Kapitel 2). Im Weiteren ist der Forschungsstand zur Handhabung sozialer Differenz zu klären, namentlich welche Forschungsperspektive eigens für die vorliegende Fragestellung formuliert werden kann (Kapitel 3). Im Empirie-Kapitel (Kapitel 4) wird dargestellt, wie die empirische Untersuchung angeleitet und durchgeführt wird und welche Ergebnisse zu vergegenwärtigen sind. Zum Schluss wird das obligatorische Fazit formuliert (Kapitel 5). Das Vorgehen soll im Folgenden genauer umrissen werden.

Im Rahmen der Studie wird untersucht, wie Stiftungen ihren Umgang mit sozialer Differenz begründen. Wie in Kapitel 2 deutlich werden wird, sind Stiftungen ganz besondere Organisationen, die als zivilgesellschaftliche Akteure in der Öffentlichkeit auftreten und sich unabhängig von anderen gesellschaftlichen Handlungsfeldern präsentieren. Ihr erklärtes Ziel ist es dabei, soziale Verhältnisse zu beeinflussen und eine Positionierung als gesellschaftspolitischer Akteur vorzunehmen. Um zu verstehen, unter welchen Bedingungen Stiftungen dabeistehen, ist es unerlässlich, ihre Verortung im gesellschaftlichen Gefüge zu erörtern. Für die Annahme, bei Stiftungen handele es sich um eine weitgehend entkoppelte Sphäre für politisches und soziales Engagement, 
spricht, dass sie neben dem „Markt“ und dem „Staat“ als Organisationen des Dritten Sektors gelten. Jedoch ist die Verortung von Stiftungen im Feld der Dritter-Sektor-Forschung nicht sonderlich eindeutig. Der Umstand, dass die Finanzierung dieses dritten Feldes zum Teil durch Zuwendungen des Staates selbst erfolgt oder eine Wechselwirkung mit dem gewinnorientierten Markt vorhanden ist, macht eine genaue Analyse der Positionierung von Stiftungen als zivilgesellschaftliche Akteure in einer weiteren Handlungsdimension nötig. Sie ist nötig, weil der Begriff der Zivilgesellschaft eine stärkere normative Konnotation hat als der deskriptive Begriff des Dritten Sektors. Hinzu kommt die Heterogenität des „Stiftungswesens“ hinsichtlich Entstehungsgrund, Budget, Verwaltungsstruktur und juristischer Rahmung, da zum Beispiel kleine Bürgerstiftungen anderen Voraussetzungen unterliegen als die in der Öffentlichkeit präsenten politischen Stiftungen. Diese und andere Herausforderungen der Verortung von Stiftungen im Spannungsfeld von Staat und Gesellschaft werden unter 2.1 erörtert. Sollen Stiftungen als zivilgesellschaftliche Organisationen des Dritten Sektors verortet werden, gilt es, die Begrifflichkeiten aufzuarbeiten. „Zivilgesellschaft“ meint, grob formuliert, dass Bürger/innen ihre Interessen in die eigene Hand nehmen und sie gegenüber der Gemeinschaft vertreten. Letztlich sind dies Werte, die Stiftungen nach außen vertreten. Um für die vorliegende Studie eine Standortbestimmung von Stiftungen vorzunehmen, soll eine Sortierung der Begriffe erfolgen, die im Kontext von Stiftungen immer wieder fallen (unter 2.2): In welcher Sphäre bewegen sich Organisationen des Nonprofit-Bereichs und der Zivilgesellschaft laut vorliegender Literatur insgesamt (2.2.1)? Welches Verständnis von Zivilgesellschaft liegt dem zugrunde (2.2.2)? Was macht Organisationen der Zivilgesellschaft aus, welche Eigenschaften werden ihnen zugeschrieben (2.2.3)?

Hiernach erfolgt in 2.3 die Skizzierung des Forschungsstands zu Stiftungen. Neben der juristischen und sozialwissenschaftlichen Definition bzw. Diskussion zu Stiftungen (2.3.1) werden im Anschluss Statistiken zur Stiftungslandschaft in Deutschland vorgestellt (2.3.2) und Forschungslücken aufgezeigt, die sich aus der Betrachtung des Forschungsstands ergeben (2.3.3).

Zum Schluss wird unter 2.4 eine Einordnung des Organisationstyps „Stiftung“ in den gesellschaftlichen Kontext vor dem Hintergrund der Erkenntnisse aus den Ausführungen in diesem Kapitel vorgenommen und ein Ausblick in Richtung der eigenen empirischen Daten gegeben.

Ist der Aspekt geklärt, mit welchen Herausforderungen die Standortbestimmung des Stiftungswesens konfrontiert ist, gilt es im nächsten Kapitel 3 den Aspekt der „sozialen Differenz“ bzw. seine theoretischen Unwägbarkeiten zu erörtern. Nicht einfach gestaltet sich der theoretische Zugang in erster Linie, weil Soziologie als Disziplin per definitionem soziale Differenz unter allen 
denkbaren Perspektiven und bezogen auf die unterschiedlichsten Handlungsfelder behandelt. Es kann aber nicht Ziel dieser Arbeit sein, eine vollständige soziologische Theorienübersicht zu präsentieren. Daher sind diverse Anhaltspunkte zu wählen, die eine sinnvolle Einschränkung der Theorienvielfalt erlauben. Wenn es darum geht, die Herstellungsweise sozialer Differenz in Hinblick auf Zielgruppen zu begutachten, die als Zielgruppe festgelegt worden sind, weil sie als gesellschaftlich benachteiligt gelten, wird die Rahmung deutlicher: Die Programmatik von Stiftungen orientiert sich an Erkenntnissen zu sozialer Ungleichheit und als Kritik an bestehenden Zuständen, von denen die Stiftung annimmt, dass sie veränderbar sind, weil sie eine Konsequenz menschlichen Handelns darstellen. Solche Prozesse von Differenzbildungen werden in ihrer basalen Form in sozialkonstruktivistischer Perspektive untersucht. Neuere sozialkonstruktivistische Ansätze betonen die Institutionalisierung von Differenzbildung, da auffällig ist, dass immer die gleichen Differenztypen zu sozialer Ungleichheit führen (vgl. Kubisch 2008: 37 ff., Wetterer 2004: 64 f.). Hauptmerkmale, die die sozialwissenschaftliche Literatur zu „sozialer Differenz" behandelt, sind Geschlecht, Ethnizität und Klasse. Diese drei Dimensionen bilden zugleich in komplexen Wechselwirkungen untereinander die Hauptkomponenten bei den bisher wissenschaftlich identifizierten sozialen Ungleichheiten (vgl. Klinger/Knapp 2008, Knapp/Wetterer 2003, BednarzBraun u. a. 2004, Meuser 2010, Berger/Schmidt 2004, Rademacher/Wiechens 2001, Weiß u. a. 2001, West/Fenstermaker 1995).

Dementsprechend folgt in diesem Kapitel zunächst eine Durchsicht sozialkonstruktivistischer Ansätze (3.2), die weniger die Frage behandeln, ob soziale Differenz gesellschaftlich konstruiert ist, sondern inwiefern sie es ist. Dieser Punkt wird in Kapitel 3.2.1 knapp umrissen, da der entsprechende Ansatz nur als Anstoß für weitere theoretische Überlegungen fungiert. Sozialkonstruktivistische Ansätze werden in erster Linie zur Kategorie Gender erfasst und stellen wichtige Anknüpfungspunkte zu menschgemachten Unterscheidungen bereit (vgl. auch Kubisch 2008: 18).

Ein weiterer Hinweis, der sich für die theoretische Rahmung ergibt, geht dahin, dass Stiftungen als organisationale Akteure agieren und zwar in Zusammenhang mit den Ungleichheitskategorien Geschlecht, Ethnizität und Klasse. Daher folgen in Abschnitt 3.2.2 die interaktionistischen Ansätze des „Un/doing difference"(West/Fenstermaker 1995; Hirschauer 2014).

Im darauffolgenden Abschnitt 3.3 werden Differenzbenennungs- und Vermengungskonzepte behandelt, die ebenfalls die Hauptachsen sozialer Differenz behandeln. Diese verraten zum einen, wie beim Forschungsgegenstand zu beobachten ist, also mehrere Differenzmerkmale gleichzeitig (anzusprechen bereits im „Doing Difference“-Ansatz von West/Fenstermaker unter 3.2.2), 
aber sie hinterfragen auch bestehende Forschungsparadigmen. So beginnt dieser Abschnitt mit dem kultursoziologischen Boundary-making- und den Othering-Ansätzen sozialer Differenz und thematisiert Grenzziehungsansätze der Kulturwissenschaften (3.3.1), da für sie jeweils die Herstellung (Kontingenz) in erster Linie dichotomer Kollektivismen und deren Fragilität im Vordergrund der Betrachtung steht. Im Rahmen der Theorien, die als Kritik an bestehenden Forschungsparadigmen formuliert werden (3.3.2), wird die postmigrantische Betrachtung der Gesellschaft als deutscher Ableger der ebenfalls kultursoziologischen Konzeption der „Hybridität“ vorgestellt. Aus der Ungleichheitsforschung wird ein Konzept gewählt, dass mehrere Ungleichheitsmerkmale gleichzeitig behandelt: Unter 3.3.3 wird der in der aktuellen Forschung stark frequentierte Topos der „Intersektionalität" hervorgehoben, der gleichzeitig versucht, die Verschränkung komplexer Ungleichheitsverhältnisse für die empirische Forschung greifbar zu machen. Hiernach wird der stärker anwendungsorientierte Ansatz der „Diversity“ skizziert (3.3.4), der als einziger auf der Meso-Ebene angesiedelt ist.

Alle vorgestellten Ansätze verfolgen ein Ziel, sie nehmen soziale Differenz als Konglomerat verschiedener Einzeldifferenzen ins Visier, fokussieren immer mehr als eine soziale Differenzkategorie und üben mehr oder minder ausgeprägt selbstreflexiv Kritik an bestehenden Forschungsparadigmen. Stand zu Beginn der Theorienbetrachtung im Rahmen der sozialkonstruktivistischen Ansätze noch die Kategorie „Geschlecht“ stark im Vordergrund, spielen bei den dichotomisierenden und verschränkenden Ansätzen die Kategorien „Migration“ und „Ethnizität" eine bedeutendere Rolle.

Unter 3.4 wird der Gehalt der genannten Ansätze verdichtet. Es wird resümiert, wie eine Forschungsperspektive zur Analyse sozialer Differenz im Falle eines organisationalen Akteurs der Zivilgesellschaft, wie es Stiftungen sind, aussehen kann.

Sind das Forschungsfeld umrissen (Kapitel 2) und der theoretische Zugang geklärt (Kapitel 3), widmet sich die Arbeit den empirischen Daten (Kapitel 4). Wie diese empirisch bearbeitet werden und welche Ergebnisse sich als tragfähig erweisen, wird in Kapitel 4 erörtert. Fokussiert wird namentlich Kubisch, wie angeführt, die habituelle Hervorbringung von sozialer Differenz, soll in der vorliegenden Studie die Benennungspraxis sozialer Differenz hinsichtlich der Stiftungsziele analysiert werden. Dies gelingt am besten mit der Befragung von Personen, die konkret an der Durchführung der Stiftungsprogrammatik maßgeblich beteiligt sind, also Projektleitende, Geschäftsführende, Programmverantwortliche. Daher sollen Experteninterviews Aufschluss darüber geben, wie die Repräsentierenden der Stiftung ihre Aktionen sowohl von der Entstehungsgeschichte als auch von der operativen Durchführung her verbalisieren. Die Auswertung des hierzu gewonnenen Materials kann dann mit jener 
Methodik gelingen, die Michael Meuser und Ulrike Nagel erstmalig 1991 benannt und im Anschluss unter verschiedenen Schwerpunkten stetig weiterentwickelt haben. Wie unter 4.1 darzulegen sein wird, wiesen bereits $1991 \mathrm{Meu}-$ ser/Nagel darauf hin, dass sie bei der Analyse „methodisch auf einem wenig beackerten Terrain operieren mußten“ (Meuser/Nagel 1991: 441). „Das gilt nahezu vollständig für Auswertungsprobleme" (ebd.). So entwickelten sie einen Vorschlag, gegliedert in fünf Schritte, wie eine Auswertung mit einem leitfadengestützten Experteninterview gelingen kann. Dieser Leitfaden (erweitert in Meuser/Nagel 2005) wurde vorliegend zur Hilfe genommen, um die Auswertung zu leisten. Zunächst steht dabei die genaue Untersuchung des jeweiligen Falls im Vordergrund, indem er paraphrasiert, sequenziert und kodiert wird. Diese Vorarbeit ermöglicht den interviewübergreifenden Vergleich, die Suche nach Homologien und Diskrepanzen, damit in der Folge eine theoretische Generalisierung gelingt (vgl. Meuser/Nagel 2005). Unter 4.2 werden die Fallauswahl und die Interviewvorbereitung (Leitfaden) beschrieben.

Die Auseinandersetzung mit den empirischen Daten beginnt in Abschnitt 4.3. Zum besseren Verständnis der Untersuchung insgesamt und der empirischen Daten im Besonderen, erfolgt zunächst jeweils die Deskription zu Personen und Situationen im Kontext der empirischen Erhebung. In 4.3.1 wird die Akquise der Interviewten und die Wahrnehmung der Interviewerin seitens der Interviewten erörtert, da davon auszugehen ist, dass sowohl die „Statusrelation“ (Meuser/Nagel 2009: 475) als auch die "Geschlechterrelation“ (ebd.) auch in der hier vorzustellenden Erhebung eine Rolle spielen. Unter Kapitel 4.3.2 wird umrissen, um was für Interviewte es sich bei den Experten genau handelt, indem ihre Tätigkeitsprofile erörtert werden. In 4.3.3 wird unter „Eigendarstellungen im Akteurshandeln" aus der Perspektive der Interviewten selbst reflektiert, wie sie sich als Wohlfahrts- und Politikakteure auto-organisational inszenieren.

Sodann erfolgt der erste Hauptblock der Auswertung. Wie in Kapitel 2 vorbereitet, werden Stiftungen als Organisationen der Zivilgesellschaft im Dritten Sektor zwischen Markt und Staat eingeordnet (vgl. Zimmer/Priller 2001). Die Daten werden entsprechend zu diesem Spannungsverhältnis näher begutachtet. In 4.3.4 wird das Verständnis von bzw. das Verhältnis zu Staat und Gesellschaft erörtert. Im Übergang zur Analyse der Herstellungsweise sozialer Differenz wird anhand von drei Fallbeispielen die Beziehungswahrnehmung gegenüber sozialen Gruppen diskutiert (4.3.5).

Diesem Abschnitt folgt das Herzstück der empirischen Auswertung. Die Fragestellung der Arbeit zielt, wie dargelegt, darauf ab, in welchen Erzählkontexten und auf welche Weise soziale Differenzkategorien und entsprechende Sichtweisen auf gesellschaftliche Verhältnisse hervorgebracht werden. Dies erfolgt unter 4.3.6 durch die entsprechende Fokussierung der Interviewdaten. 
Dabei werden konkrete soziale Grenzziehungen dahingehend fokussiert, wie die Auswahl der Zielgruppen erfolgt ist, wie sie sich gegenüber den Zielgruppen positionieren und welche gesellschaftlichen Zielvorstellungen damit verbunden werden. Unter 4.4 werden die Argumentationskonstellationen in Hinblick auf die konkreten Differenzmerkmale aufgearbeitet.

Im Fazit (Kapitel 5) wird unter 5.1 rekapituliert, welche Ziele mit der vorliegenden Studie verbunden waren und wie bis zu diesem Punkt vorgegangen wurde. Die gewonnenen Erkenntnisse werden hiernach unter 5.2 aus einer weiteren Perspektive zu einer theoretischen Generalisierung verdichtet. Dies geschieht unter Hinzunahme aller Erkenntnisse aus der Auswertung des empirischen Materials. Es werden zwei Ziele verfolgt: Zum einen wird erarbeitet, als welche Art Organisation sich Stiftungen in Hinblick auf ihre gesellschaftliche Rolle präsentieren. Namentlich das reziproke Verhältnis zum Staat macht Stiftungen ganz speziell, ihm soll in diesem Kapitel ein besonderes Augenmerk gelten. Fraglich ist, ob sich zudem Erkenntnisse zum Organisationstyp Stiftung erweitern lassen. Wie kann die gesellschaftliche Verortung präzisiert werden, die auch Visionen und normierende Grundsätze einschließt? Zum anderen gilt das Interesse der Frage, welche Wirkebenen mit welchen sozialen Gruppen rekonstruiert werden können. $\mathrm{Zu}$ fragen ist hier, ob Muster bei der Benennungspraxis sozialer Differenz zu erkennen sind. Können Wechselbeziehungen zwischen Ungleichheitsmerkmalen beobachtet werden, die in öffentlichen Diskursen selten gemeinsam gedacht werden?

Schlussendlich werden die Ergebnisse unter 5.3 mit Blick auf ihre Übertragbarkeit und Anschlussfähigkeit diskutiert, um auch in anderen sozialen Sphären und auf anderen Ebenen die Funktionsweise von „Humandifferenzierung“" (Hirschauer) eruieren zu können. 


\section{Stiftungen im Spannungsfeld von Staat und Gesellschaft}

In diesem Kapitel soll, bevor das Verständnis geklärt wird, was mit sozialer Differenz gemeint ist (Kapitel 3), zunächst der gesellschaftliche Akteur Stiftung genauer betrachtet werden. Dabei liegt der Schwerpunkt auf der sozialwissenschaftlichen Konzeptionierung dieses speziellen Organisationstyps und weniger auf der Deskription infrastruktureller Daten. Denn wenn die Frage behandelt wird, wie Stiftungen mit sozialer Differenz umgehen, ist die Verortung ihres Wirkungsbereichs relevanter als rein statistische Deskriptionen zu Stiftungen. Bei der Konzeptionalisierung des Stiftungswesens besteht sozialwissenschaftlich gesehen insgesamt noch viel Nachholbedarf. Dennoch kann das Stiftungswesen in Deutschland trotz vorhandener Forschungslücken auf einen Nenner gebracht werden, um sie als organisationale Akteure diskutieren zu können, die soziale Projekte mit mannigfaltigen Zielgruppen initiieren und somit direkt oder indirekt soziale Differenz mitmarkieren. Diese bestehen zum einen in den Herausforderungen, die mit dem Versuch verbunden sind, ihren gesellschaftlichen Standort zu bestimmen (2.1). Zum anderen wird zu zeigen sein, dass Stiftungen als besondere Organisationen des Dritten Sektors bzw. der Zivilgesellschaft gesehen werden können. Dafür werden zunächst die Konzeptionen rund um die begrifflichen Zugänge geklärt (2.2), worauf die Charakteristika des deutschen Stiftungswesens skizziert werden (2.3), um abschließend die gesellschaftliche Standortbestimmung vornehmen zu können (2.4).

\subsection{Standortbestimmungen im gesellschaftlichen Kontext}

Bei dem Versuch Stiftungen als zivilgesellschaftliche Organisationen in den Kontext „Staat“ und „Gesellschaft" einzubetten, gilt es, Akteurskonstellationen mit Handlungsfeldern einerseits und die Einordnung in die öffentliche Sphäre andererseits zu gliedern. Denn Stiftungen umspannen in ihrer gesellschaftlichen Funktionsweise bisweilen ungewöhnliche Ambivalenzen: Sie sind elitär konzipiert, möchten sich aber am Allgemeinwohl ausrichten. Sie sind demokratisch nicht durch Wahlen legitimiert, greifen aber in staatliches Handeln ein bzw. übernehmen Aufträge in der sozialen Fürsorge vom Staat oder betätigen sich als politische Akteure. In einer Zukunftsvision formuliert Adloff, dass Stiftungen ,,zu Kristalisationskernen der Zivilgesellschaft' werden könnten, da sie prinzipiell in der Lage wären, eine Kontinuität von Inhalten von den tagespolitischen Konjunkturen sicherzustellen“ (2004: 282). Deutsche 
Forscherinnen und Forscher stellen recht konstant fest, dass für die Sicherstellung der Legitimation, der Integrität und der Reputation von Stiftungen gerade im internationalen Vergleich gewisse Ansprüche hinsichtlich der Transparenz und Kontrollmechanismen unzureichend geblieben sind (vgl. Wolf 2009: 70). Auch wichtige wissenschaftliche Analysen fehlen bzw. es werden Forschungslücken erst allmählich durch Publikationen der jüngeren Vergangenheit geschlossen (vgl. Anheier u. a. 2017a, Anheier u. a. 2017b, Anheier u. a. 2017c, Striebing 2017, Meyer 2015, Strachwitz 2015b, Strachwitz 2016).

Neben den beschriebenen Ambivalenzen der Handlungslogik kommen diverse Aspekte hinzu, die Stiftungen als eine sehr spezielle Organisationsform des Dritten Sektors und der Zivilgesellschaft und letztlich im gesamtgesellschaftlichen Gefüge zu beschreiben erlauben: Es zeigt sich eine starke Vielfalt bei der Konzeption der Organisationsform (Anheier 2001) sowohl hinsichtlich der juristische Konstruktion wie auch als sozialwissenschaftliches Phänomen. In der Diskussion um „die Reform und Modernisierung der überkommenen Verhältnisse von Staat und Verwaltung“ (Zimmer 2002b: 42) identifiziert Annette Zimmer im Kontext von Bürgerengagement, Drittem Sektor und Zivilgesellschaft drei Diskurse: einen demokratietheoretischen, einen wohlfahrtsstaatlichen sowie einen verwaltungswissenschaftlichen Diskurs (vgl. Zimmer 2002b: 42). Während es im ersteren im weitesten Sinne um politische Teilhabe geht, bezieht sich der zweite Diskurs auf Tätigkeiten von Individuen und Organisationen, die auf das Gemeinwohl ausgerichtet sind. Der verwaltungswissenschaftliche Diskurs zielt auf die Ergänzungsfunktion (Anheier) von zivilgesellschaftlichen Organisationen mit Blick auf staatliche Güter und Dienstleistungen (vgl. Zimmer 2002b: 42 f.). Es kann vorweggenommen werden, dass Stiftungen mit ihren monetären Leistungen sowie ihrem operativen Vorgehen alle drei Felder durchkreuzen. Hinzu kommt global betrachtet ein im Vergleich zur Gesamtbevölkerung sehr großes Stiftungswesen in Deutschland, möglicherweise ${ }^{2}$ das zweitgrößte nach dem der USA (vgl. Anheier u. a. 2017a: $12 \mathrm{f}$.).

Neben den oben angedeuteten, in ihrer Zahl überschaubar wenigen theoretischen Überlegungen zum Organisationstypus der Stiftung reihen sich einige wenige Erkenntnisse aus quantitativen empirischen Studien zum Umfang des Stiftungswesens (vgl. Bundesverband Deutscher Stiftungen 2011), zu Motiven von Stiftungsgründenden (Timmer 2005) sowie eine systematische ErschlieBung von Motiven und Eigeneinschätzungen bei der Abgrenzung zu staatlichen und privatwirtschaftlichen Akteuren (Anheier u. a. 2017a, Anheier u. a. 2017b, Anheier u. a. 2017c) ein. Ziel der vorliegenden Untersuchung ist es nicht zu erörtern, welchen Einfluss Stiftungen durch ihre Handlungen tatsäch-

Dem Autor ist es wichtig, diese Einschätzung als Vermutung anzugeben. 
lich haben oder welche Veränderungen sie messbar bewirken. Durch ihre spezifische Verfasstheit, die im Fortgang dieses Kapitels näher erläutert wird, bilden sie in einem anderen Kontext ein interessantes Forschungsfeld: Man gehe davon aus, im Stiftungskontext könnten Narrationen zu gesellschaftspolitischen Einschätzungen aus sich selbst heraus oder als Reaktion auf bestehende Zustände gebildet werden und zirkulieren, da Stiftungen in ihrer Handlungslogik nicht den ,typischen' Sphären ,Markt' und ,Staat' unterworfen sind (vgl. Adloff 2004: 280), wie im Laufe des Kapitels zu klären sein wird. Damit eröffnet sich eine besondere Perspektive auf soziale Differenz, die es an dieser Stelle weiter herauszuarbeiten gilt.

\subsection{Zentrale Begriffe}

Im Rahmen des Forschungszweigs, der sich Vereinen, Freiwilligenarbeit, gemeinnützigen Organisationen, Wohlfahrtsverbänden oder anderen gemeinnützigen Geldgebenden bzw. Dienstleistenden annimmt, werden auf relevanten Analyseebenen Begriffe wie „Dritter Sektor“, „Bürgerschaftliches Engagement", „Nonprofit Organisationen“ (NPO) oder „Zivilgesellschaft“ herangezogen, die nicht immer trennscharf definiert werden können (vgl. Sprengler u. a. 2011: 7 f.). Allerdings lassen sich die Konzepte mittlerweile gut sortieren und verschiedenen Forschungslogiken zuordnen.

Während Bürgerengagement auf die Mikro-Ebene und die individuellen Aktivitäten abzielt, und der Dritte Sektor sich vorrangig auf die Meso-Ebene der meist mitgliedschaftlichen Organisationen (Vereine) bezieht, wird mit der Zivilgesellschaft die gesellschaftliche Dimension und damit die Makro-Ebene angesprochen (Priller/Zimmer 2000: 3).

Stiftungen mit sozialen Projekten bewegen sich somit im Bereich des Dritten Sektors und der Zivilgesellschaft, da Stiftungen vordergründig hauptamtlich Mitarbeitende mit Gehalt beschäftigen und somit nicht in den Bereich des (unentgeltlichen) Bürgerengagements fallen. In den folgenden Ausführungen geht es daher um die Frage, welche spezifischen Charakteristika die Konzepte „Dritter Sektor“ (2.2.1) und „Zivilgesellschaft" (2.2.2) ausmachen, um sodann ein erstes Resümee im Hinblick auf den gesellschaftlichen Standort des Stiftungswesens zu ziehen (2.2.3). 


\subsubsection{Der Dritte Sektor-Ansatz}

Der Dritte Sektor erhielt seine Bezeichnung, weil er in Abgrenzung zum (gewinnorientierten) Markt und zum staatlich organisierten öffentlichen Sektor auftritt (vgl. u. a. Birkhölzer 2005: 75; Zimmer 2002a: 2; Zimmer/Priller 2007: 52 f.; Priller/Zimmer 2000: 2). Er ist nicht zu verwechseln mit dem volkswirtschaftlichen Begriff des „tertiären Sektors“ (Birkhölzer 2005: 72), dem Dienstleistungssektor. Der Sektor-Begriff erhält seine Berechtigung vordergründig neben der zunehmenden ökonomischen, politischen und sozialen Bedeutung aufgrund seines ,ausgleichenden Charakters", wie nachfolgend darzustellen sein wird. Der US-amerikanische Soziologe Amitai Etzioni definierte den Dritten Sektor (und seine Abgrenzung) bereits 1973:

While debate over how to serve our needs has focused on the public versus the private alternative, a third alternative, indeed sector, has grown between the state and the market sector. Actually this third sector may well be the most important alternative for the next few decades, not by replacing the other two, but by matching and balancing their important roles (Etzioni 1973: 315, Hervorhebung aufgehoben).

Der Staat stellt Güter und Dienstleistungen zwar wie der Dritte Sektor nicht profit-orientiert und zum Allgemeinwohl bereit, ist dabei aber dem Gleichheitsgebot, dem Zwang zur Machtausübung sowie strengen bürokratischen Regularien unterworfen. Demgegenüber lässt sich der profitorientierte Markt positionieren, der zum öffentlichen Sektor ergänzend auftritt oder aber völlig andere, neue Produkte und Dienstleistungen bereitstellt. Die Sphäre der Privatheit markiert die Familie bzw. familiales Zusammenleben und Gemeinschaft. Der deskriptive Dritte Sektor-Begriff dient in erster Linie als „heuristisches Modell“" (Zimmer 2002a: 2), das eine hohe Anschlussfähigkeit zur (quantitativen) empirischen Forschung sowie zur Theoriebildung besitzt (vgl. Zimmer 2002a: 7 f.). Dabei wird ein Feld markiert, das fließend angelegentlich eine Nähe zum Markt, zum Staat oder zur Privatheit aufweist (siehe Abbildung 1). 
Abbildung 1: Der Dritte Sektor als heuristisches Modell

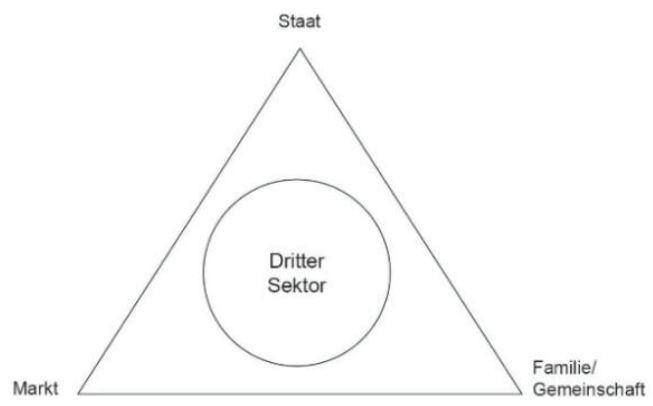

(Quelle: Zimmer 2002a: 2)

Somit umfasst der heterogene Dritte Sektor eine ganze Bandbreite an Organisationen mit je eigener Ausrichtung, weshalb von einer Heuristik gesprochen werden muss, da kaum alle Initiativen, informellen Vergemeinschaftungen oder nicht-öffentliche Selbsthilfegruppen bürokratisch und statistisch erfasst werden können. Die Organisationen des Dritten Sektors, die sogenannten Nonprofit-Organisationen, haben laut Salamon/Anheier in Deutschland historisch bedingt ein kooperativ-interdependentes Verhältnis zum Staat (vgl. Salamon/Anheier 1997: 227). Gerade die Abgrenzung zum Staat erfuhr ein besonderes Augenmerk, dem sich die Sozialwissenschaft seit den 1970er Jahren im Rahmen von Diskussionen zur „Veränderung von Staatlichkeit“ (Zimmer 2002a: 3) verschrieb. So werden Dritte-Sektor-Organisationen, ,als Alternative zu einer rein staatlichen Aufgabenübernahme“ (Zimmer 2002a: 4) sowie als ,wichtige Akteure im Rahmen des Agendasetting und der Politikformulierung betrachtet" (ebd.). Die Gegenüberstellung mit dem Staat kann sehr variieren: Mal tritt der Dritte Sektor als „Kontrollinstanz“ (zum Beispiel Bürgergruppen, die Interessen gegenüber staatlichen Behörden behaupten), mal in „Coexistenz" (zum Beispiel Kleingärtnervereine neben öffentlichen Parkanlagen), ein anderes Mal als Kooperationspartner (zum Beispiel Freiwilligenagenturen, die Freiwillige in die Vereine vermitteln) auf. Der Dritte Sektor hat dabei eine eigene, auf individuelle Entscheidungen beruhende (vgl. Zimmer/Priller 2007: 16) Handlungsmotivation.

Der entscheidende Unterschied zwischen dem Dritten und den anderen beiden [Staat und Markt, Anm. NDS] Sektoren besteht jedoch darin, dass nur im Dritten Sektor die Solidarität als Motiv, Motivation sowie als Medium der Handlungskoordination von Mitgliedern, Mitarbeitern sowie Förderern einen zentralen Stellenwert besitzt (Birkhölzer u. a. 2005: 10). 
Dass Solidarität als Handlungsmotiv gilt, macht den Dritten Sektor zu einem intrinsisch motivierten, anhand normativer Vorgaben handelnden organisationalen Akteursbündel und in gewisser Weise zum stabilisierenden „Bindeglied zwischen Individuum und Gesellschaft“ (Zimmer 2002a: 9). Sie lenkt den Blick vom deskriptiv-heuristischen Charakter des Dritte-Sektor-Ansatzes hin zum Zivilgesellschafts-Begriff, der, wie oben erwähnt, mehr Bedeutungsebenen erfasst als die reine „Meso-Ebene“ (Priller/Zimmer 2000: 3) an NonprofitOrganisationen - zum einen. Während allerdings ,der zivilgesellschaftliche Diskurs Assoziationen ausschließlich von der Input-Seite des politisch-administrativen Systems thematisiert" (Priller/Zimmer 2000: 4), also in Etzionis Sektoren-Triade aus dem staatlichen Blickwinkel das Verhältnis zu organisierter Freiwilligkeit fokussiert, erfasst der Dritte-Sektor-Ansatz zum anderen mehr als nur diese Perspektive (vgl. Priller/Zimmer 2000: 4/5). Daher eignet sich letzterer unter anderem besonders für die quantitativen Deskriptionen, die die empirische Dritte-Sektor-Forschung im Kern ausmachen. ${ }^{3}$ Allerdings muss sich der Dritte-Sektor-Ansatz nicht auf diesen Zugang beschränken, da Organisationen des Dritten Sektors sehr wohl auch zivilgesellschaftliche Akteure sein können. (vgl. ebd.) Die Offenheit des Konzepts ist neben seiner Stärke daher gleichzeitig seine Schwäche. Sie ermöglicht weniger eine fokussierte Klärung darüber, in welchen Spannungsfeldern bestimmte Akteurs(gruppen) des Dritten Sektors stehen und welche Erkenntnisse diese für eine weitergehende (qualitative) empirische Forschung bereithalten.

\subsubsection{Das Konzept der Zivilgesellschaft}

Unter 2.2.1 wurde dargelegt, dass, wie beispielsweise prominent von Böckenförde formuliert, Gesellschaft in der deutschen ideengeschichtlichen Tradition als Differenzbegriff zum Staat verstanden wird (vgl. Pascher 2001: 10) und dass Zivilgesellschaft in der gesellschaftlichen Makro-Ebene (vgl. Priller/Zimmer 2000: 3) zu verorten ist, womit besonders das Verhältnis zum öffentlichen Sektor in den Blick kommt.

Um die Ansätze und Spannungsfelder rund um das Konzept der Zivilgesellschaft nachvollziehen zu können, ist ein kurzer Blick Richtung der philosophisch-historischen Denktradition und Genese hilfreich.

So führt Adloff (2005: 17 ff.) aus, dass der Begriff „Zivilgesellschaft“ oder „bürgerliche Gesellschaft“ auf Aristoteles (4. Jahrhundert v. Chr.) zurückzuführen ist und im Lateinischen als „,societas civilis“ in der Literatur erscheint.

3 Zum Beispiel Entwicklung der Zahlen zu Neugründungen von Vereinen, Stiftungen und Verbänden in den letzten Jahrzehnten; Entwicklung der Zahlen zu hauptamtlichen Mitarbeitenden in Vollzeitäquivalenten und Umrechnung in volkswirtschaftliche Kennzahlen; Motive Ehrenamtlicher usw. 
Es bedeutet ,wörtlich übersetzt so viel wie Bürgervereinigung oder Bürgergemeinde" (vgl. ebd.). Dahinter verbirgt sich der Gedanke, dass freie Bürger in der athenischen Bürgergemeinde (polis) in Eigenregie über Belange der Gemeinschaft entscheiden. Frauen und Versklavte bzw. Bevormundete waren hier exkludiert und allen voran dem Privathaushalt oder der ökonomischen Sphäre zugewiesen. Nach einer in den folgenden, vormodernen Jahrhunderten zu beobachtenden Konkurrenz weltlicher und geistlicher Machtansprüche in philosophischen Abhandlungen zu den Staatskonzepten, nahm der Diskurs zur Rolle des „Bürgers im Staate“ bei Thomas Hobbes (1651) eine interessante Wendung: Die „Idee vom Bürger als eines Menschen, der sein eigener Herr ist, [hat] keine Bedeutung mehr" (Adloff 2005: 21). Alle Bürger sind dem Staate unterworfen, dem die Aufgabe zugeteilt wird, für Recht und Ordnung zu sorgen, also ein Machtmonopol zu bilden. „Die Bürgergesellschaft ist damit apolitisch geworden“ (ebd.). Während der Staat Macht ausübt und Gesetze durchsetzt, die das weltliche Zusammenleben regeln, schafft er einen privaten Raum, der ihn nicht mehr interessiert. Gesinnung ist nun Privatsache geworden (vgl. Adloff 2005: 22). Wie dieser, private Bereich` durch den Staat oder vor dem Staat geschützt werden soll, wie sie sich zueinander positionieren und welche Kooperationen denkbar sind, ist bis heute Gegenstand zahlreicher Diskurse.

Fraglich war, wie sich staatliche Ordnung ,ohne Rekurs auf Gott als höchste Instanz" (Adloff 2005: 22) aus sich selbst begründen sollte. Diese Frage stellte sich in einem ähnlichen historischen Kontext wie Hobbes auch John Locke („Two Treatises of Government“, 1690). „Das moderne Individuum tauchte damit langsam [wieder, Ergänzung NDS] am geistesgeschichtlichen Horizont auf" (Adloff 2005: 22), da klar wurde, dass die Wechselbeziehung von „Staat" und „Bürgergesellschaft“ nur durch mündige, gut informierte Bürger, unabhängig von überirdischen Referenzen dauerhaft getragen und ausgestaltet werden kann. Auch wenn damit direkt der Blick auf Kants Postulat vom Ausgang des Menschen aus seiner selbstverschuldeten Unmündigkeit gerichtet wird, soll als nächster Meilenstein Hegel genannt werden, der Kants Ausführungen zum mündigen Individuum aus der Mikro-Ebene in eine staatsphilosophische Konzeption integrierte („Grundlinien der Philosophie des Rechts“, 1821). Unter anderem heißt es dort:

[...] der Familie und der Bürgerlichen Gesellschaft ist der Staat einerseits eine äußerliche Notwendigkeit und ihre höhere Macht, deren Natur ihre Gesetze, sowie ihre Interessen untergeordnet und davon abhängig sind; aber andererseits ist er ihr immanenter Zweck und hat seine Stärke in der Einheit seines allgemeinen Endzwecks und des besonderen Interesses der Individuen, darin, dass sie insofern Pflichten gegen 
ihn haben, als sie zugleich Rechte haben [...] (Hegel 1821 [1995]: § 261 [215], Hervorhebungen wie im Druck von 1995).

Hegel stellt neben der Verfasstheit von „Staat“ und „Bürgergesellschaft“ nun die Reziprozität dieser beiden Bereiche heraus. Somit reproduzieren sich in dieser Logik die Sphären „Bürgergesellschaft“ und „Staat" gegenseitig bzw. die Existenz des Staates ist für eine gelungene Bürgergesellschaft unerlässlich, dies gilt aber auch umgekehrt. Der Staat gewährleistet Sicherheit und Ordnung, damit Privatheit möglich und geschützt ist („Familie“) und eine aktive Bürgerschaft überhaupt gestalten und prägen kann. Andererseits ist der Staat darauf angewiesen, dass sein Machtmonopol von den „Bescherrschten“ nicht in Frage gestellt wird bzw. sein Machterhalt sogar gestützt wird. Der Staat hat ein Interesse daran, dass eine (einigermaßen) starke Bürgergesellschaft erhalten bleibt, „um die Unterstützung wichtiger gesellschaftlicher Eliten $\mathrm{zu}$ sichern und gleichzeitig radikaleren sozialreformerischen Forderungen die Basis zu entziehen" (Salamon/Anheier 1997: 227) Im damaligen Verständnis war die Gruppe der Bürger auch nicht identisch mit der Bevölkerung des Landes. Mitglieder der bürgerlichen Gesellschaft sind laut Hegel ,Wirtschaftsbürger [...]“ (Adloff 2005: 32), die eigenen Interessen nachgehen (vgl. ebd.). Das Konzept gilt trotz der sozialen Auslese der Protagonisten als sozialintegrativ, natürlich mit Abstrichen gegenüber unserem modernen Verständnis von sozialer Gleichheit, bildet doch die Korporation den Kern der bürgerlichen Gesellschaft, in der „,die Mitgliedschaft nicht auf askriptiven oder erblichen Kennzeichen, sondern auf Freiwilligkeit beruhen solle“ (Adloff 2005: 33). Es entsteht eine Verbindung zwischen dem eigennützig agierenden Homo oeconomicus und dem auf das Gemeinwohl ausgerichteten Bürgerhandeln, zugegebener Maßen weiterhin als bürgerlich-männliches Phänomen (vgl. Kocka 2002). Nachfolgend (zum Beispiel bei Alexis de Tocqueville: „De la démocratie en Amérique“, 1835) wird die immense Bedeutung dieses Bürgerverständnisses für demokratische Gesellschaftsordnungen diskutiert, ebenfalls bis heute.

Der knappe Aufriss zur Genese des Zivilgesellschaftsbegriffs stellt Weichen für die nachfolgende Diskussion: Zum einen verbirgt sich hinter der Theorie, was den Staat und die Bürger ausmacht, ein sehr spezifisches Bild einerseits davon, was Menschen können und was sie dürfen sollten. Es illustriert auf der anderen Seite, dass die Zivilgesellschaft immer korrespondiert zu einem bestimmten Verständnis von staatlicher Herrschaft. Dieses Wechselspiel ist epochalen politisch-historischen Kontexten unterworfen und taucht in zyklischen Hochs und Tiefs gelegentlich positiv besetzt mal zugunsten des einen, mal zugunsten des anderen auf. Die Herausstellung des wirtschaftlich starken, informierten Individuums, das sich interessehalber mit seinesgleichen zusammenschließt, bildet den Kern der Ansätze rund um Zivilgesellschaft. Mit dem 
Begriff der „Zivilität“ wird normiert, dass „Höflichkeit, Toleranz und Vertrauen [...] zum Charakteristikum“ (Adloff 2005: 94 f.) notwendig zu einer gelungenen Bürgergesellschaft gehörten.

Wie viel Staat mit wie viel Zivilgesellschaft korreliert, schätzen die einschlägigen Autorinnen und Autoren recht unterschiedlich ein. Während Adloff aus einem US-amerikanischen Blickwinkel die Vorstellung: „Je weniger Staat, desto mehr Nonprofit-Organisationen" (Adloff 2005: 109), diskutiert, wird im europäischen Kontext oftmals die Bedeutung von aktiver Bürgerschaft im Kontext veränderter (schwächerer) Staatlichkeit (Zimmer 2002b, Benz 2001) betont. Dabei ergibt sich eine Dichotomie der Zivilgesellschaft als ,Gegenmacht ${ }^{\star}$ gegenüber staatlichen Instanzen und Regierung sowie die „Sphäre des bürgerlichen Diskurses zur Lösung von Problemen allgemeiner Bedeutung““ (Zimmer 2002b: 42), die aber nicht scharf abgetrennt konzipiert sein muss. Insbesondere der Blick in die zivilgesellschaftliche Praxis zeigt, dass dieses Verhältnis komplexer zu sehen ist. So kann gleichzeitig eine Stärkung zivilgesellschaftlicher Akteure dadurch beobachtet werden, dass der Staat wichtige Aufgaben, zum Beispiel in der sozialen Fürsorge, an Nonprofit-Organisationen delegiert, samt der hierzu notwendigen finanziellen Mittel (vgl. Adloff 2004: 274 f.). Für politische Handlungen ist damit auch die Frage entscheidend, ,wo und wie das selbstorganisierte Handeln in der Zivilgesellschaft und das demokratisch legitimierte hoheitliche Handeln des Staates ineinandergreifen“" (Strachwitz 2015a: 43).

\subsubsection{Quo vadis Zivilgesellschaftsforschung}

Zwar hat sich die Zivilgesellschaft weitestgehend auch als Forschungsrichtung etabliert, obwohl hier, eine ausformulierte sozialwissenschaftliche Theorie der Zivilgesellschaft fehlt" (Kocka 2001: 21). Dennoch ist das Forschungsfeld durch sehr viel Dynamik geprägt (vgl. Akkaya 2012: 45). Waren Diskussionen zur Zivilgesellschaft in den vorangehenden Jahrzehnten eher als starker Gegenpol gegenüber staatlicher Obrigkeit konnotiert, stehen aktuell eher kooperierende Bewältigungsstrategien von Zivilgesellschaft und öffentlichem Sektor mit Blick auf die Wohlfahrtsstaatlichkeit im Vordergrund (vgl. Priller 2002: 40). Einer mühelosen empirischen Operationalisierung entzieht sich das Konzept aufgrund seiner schillernden Bedeutungszuweisung (vgl. Krimmer u. a. 2014: 7). Insoweit ist das Dritte-Sektor-Konzept einschlägig. Der große Gewinn zivilgesellschaftlicher Forschungen speisen sich daraus, dass das Konzept ,eine Antwort auf die Frage [liefert], was freiwillige Vereinigungen leisten müssen, damit sich demokratische Gesellschaften weiter entwickeln können“ (Anheier/Priller/Zimmer 2000: 77) Somit haben zivilgesellschaftliche 
Organisationen eine Stabilisierungsfunktion. Hierzu kann eine Definition formuliert werden, die in der hergeleiteten Argumentationskonstellation das Forschungsfeld für die vorliegende Studie greifbar macht. Es wird resümiert:

Organisationen der Zivilgesellschaft sollen als Feld aller formell und informell freiwillig und solidarisch organisierten Personen und Institutionen verstanden werden, deren Handlungslogik im ökonomischen Sinne nicht-gewinnorientierten Vorgaben folgt. Dabei rekurriert die nachhaltige Zusammenkunft auf ein klar definiertes Interesse (und zum Beispiel nicht entlang askriptiver Merkmale von Mitwirkenden). Die Aktivitäten finden im Rahmen der Rechtsordnung statt, sie haben aber nicht die Aufgabe, diese zu reproduzieren, wie es staatliche Akteure tun. Gleichwohl sind sie als ein gesellschaftlicher Stabilisierungsfaktor zu sehen.

In Hinblick auf Stiftungen werden ferner zwei Aspekte genauer zu klären sein, die auf das Verhältnis gegenüber den anderen beiden Sektoren abzielen:

(1) Inwiefern ist der „Markt“ Teil dieser Definition? Wenn Unternehmen unter dem Label „Corporate Social Responsibility“ soziale Projekte durchführen, ganze Nonprofit-Ausgründungen für solche Projekte veranlassen oder Stiftungen gründen, befinden sie sich, bildlich gesprochen, mit mindestens einem Bein in der Zivilgesellschaft, obwohl die Finanzierung dieser Aktivitäten über gewinnorientiertes unternehmerisches Handeln gewährleistet wurde. Das gleiche gilt umgekehrt für zivilgesellschaftliche Akteure, die herkömmliche unternehmerische Arbeitgeber sein können und tariflich angemessene Gehälter an Angestellte zahlen, wie es beispielsweise Wohlfahrtsverbände tun. In einer Abhandlung rechnet Keane („Global Civil Society?“, 2003) aus einer angelsächsischen Perspektive den „Markt“ der Zivilgesellschaft zu, was aus europäischer Sicht recht befremdlich erscheint. ${ }^{4}$

(2) Der zweite Fokus richtet sich auf die Frage, wie staatliche (Teil-) Finanzierungen von Organisationen zu werten sind, die Bürgerinteressen vertreten (zum Beispiel die Verbraucherzentralen) oder sich als Akteure der Zivilgesellschaft verstehen, zu denen die parteinahen Stiftungen hinzugerechnet werden müssen. Beispielsweise betrug der Anteil an Zuwendungen des Bundes an sogenannte parteinahe Stiftungen im Jahr 2010 etwa 400 Millionen Euro (vgl. Klaassen 2016: 65). Die vielbeachtete Johns-Hopkins-Studie deckte auf, dass bei 23 Prozent der deutschen Nonprofit-Organisationen die Finanzierung zu mehr als 50 Prozent auf die öffentliche Hand zurückgeht (vgl. Priller 2002: 44).

4 Siehe Buchbesprechung Helmut K. Anheier in: American Journal of Sociology, 2004, Vol. 110/1, p. 234-235. 


\subsection{Forschungsstand zu Stiftungen}

Die konkreten Hinweise aus der Literatur zur Frage, wie und unter welchen Kriterien Stiftungen in die Landschaft der Dritte-Sektor- bzw. zivilgesellschaftlichen Organisationen einzuordnen sind, sind gering an Zahl. Sie können in einen theoretischen und in einen empirischen Forschungszweig unterschieden werden, wobei letzterer vor allem sehr aktuelle Studien umfasst. Zunächst sollen hier definitorische Weichen gestellt und Ansätze einer möglichen Stiftungstheorie besprochen werden (2.3.1). Darauf folgt die knappe Aufarbeitung des vorhandenen Zahlenmaterials zu Stiftungen in Deutschland (2.3.2). Zu guter Letzt werden die Befunde verdichtet und Forschungslücken aufgezeigt (2.3.3).

\subsubsection{Was ist eine Stiftung?}

Der Terminus Stiftung ist gesetzlich nicht eindeutig festgelegt (vgl. Bundesverband Deutscher Stiftungen 2011: 16), es handelt sich mehr um ein Etikett, mit dem sich hauptsächlich gemeinnützige Organisationen benennen. Als „Prototyp” (Bundesverband Deutscher Stiftungen 2011: 16) können sogenannte rechtsfähige Stiftungen des nach $\S 80 \mathrm{ff}$. Bürgerliches Gesetzbuch (BGB) gelten. Die juristische Perspektive rahmt Stiftungen als Organisationen, die einen Namen (1), einen Stiftungssitz (2), einen Stiftungszweck (3), ein Vermögen (4) sowie einen Vorstand (5) haben müssen (§ 81 BGB), damit sie als solche in die bürokratischen Strukturen einverleibt, der staatlichen Stiftungsaufsicht unterstellt und in den meisten Fällen die begehrten steuerlichen Vorzüge geltend machen können. 94 Prozent der Stiftungen können statistisch hier einsortiert werden (vgl. Bundesverband Deutscher Stiftungen 2011: 17), daneben gibt es noch einige Stiftungen, die privatnützig agieren (vgl. ebd.), wie es beispielsweise Familienstiftungen sind. Eine Stiftung wird hierbei als eine eigentümerlose Vermögensverwaltungsanstalt gesehen. Historisch zählten hierzu insbesondere die Fideikommisse, vor allem adeliger Familien. Untypisch, aber dennoch bedeutsam, sind die Stiftungsgründungen in Form von anderen juristischen Personen privaten Rechts, zum Beispiel als (gemeinnützige) Gesellschaften mit beschränkter Haftung $(\mathrm{gGmbH})$ oder als eingetragener Verein (e.V.), wie es bei unternehmensnahen oder parteinahen Stiftungen häufiger vorkommt. Ihre Vorteile werden in der Flexibilität der Finanzverwaltung und in der operativen Arbeit gesehen, weil sie einfacher umstrukturiert oder aufgelöst werden können, da auf sie kein Stiftungsrecht angewandt wird (vgl. Bundesverband Deutscher Stiftungen 2011: 17). Sind Bürgerstiftungen bereits ei- 
nige Jahrhunderte alt, ist eine treuhänderische Verwaltung durch einen öffentlichen Träger in Form von ,kommunalen Stiftungen“ nicht unüblich (vgl. Bundesverband Deutscher Stiftungen 2013a zit. nach Bundesverband Deutscher Stiftungen 2013b: 19), jedoch bleibt der Stifterwille strikt einzuhalten.

Das Wesen einer Stiftung allein auf ihre juristische Definition zu fixieren, wäre eine allzu starke Vereinfachung. Mit dieser Rahmung ist die Frage, was eine Stiftung ist, bei weitem nicht geklärt. Es bleibt neben der Herausforderung zu klären, ,wie eine Stiftung als soziales Phänomen zu definieren ist" (Strachwitz 2015b: 283), genauso die Frage offen, wie die „soziale Realität“" (Strachwitz 2015b: 288) in ihrem historisch-gesellschaftlichen Kontext zu verstehen ist. Eine Einordnung hierzu steht vor der Herausforderung, dass dieser Forschungsbereich von eklatanten Forschungslücken geprägt ist. Strachwitz spricht sogar davon, dass es seit ,100 Jahren [...] in Deutschland kaum größere öffentliche oder wissenschaftliche Debatten um die Stiftungen gegeben“" (vgl. Strachwitz 2015b: 283) habe.

Einen tieferen Einblick in das soziale Phänomen „Stiftung“ erlaubt eine Abhandlung von Mohammed Rassem aus dem Jahr 1956, ${ }^{5}$ auf die auch Strachwitz zurückgreift. Rassem lenkt den Blick auf den individuellen Stiftungsursprung, wenn er schreibt:

Konstituierend ist ein Akt der Stiftung oder die Vorstellung, daß es einen solchen Akt gegeben habe. Eine Wesensbestimmung der Sozietät ist also: sie hat einen Anfang; sie und ihre Geschichte ,stehen in einem Zeichen'. Es wird deshalb unser Verständnis fördern, wenn wir gerade solche Gemeinschaften betrachten, deren Stiftungsakt und Gestiftetsein besonders ausgeprägt ist und bleibt (Rassem 1960: 492).

Strachwitz greift diesen Gedanken auf, wenn er seine Ausführungen dahingehend präzisiert, dass ,,alle Stiftungen dadurch definiert“ seien, „dass sie an den bei der Gründung niedergelegten Willen des Gründers oder der Gründer für die Dauer ihres Bestehens gebunden bleiben“" (Strachwitz 2015b: 288 f.) Beide, Rassem und Strachwitz, stellen letztlich fest, dass nicht nur Geldmittel als Vermögen in Betracht kommen, sondern in Rekurs auf die Entstehung der monotheistischen Religionen eine Vision oder eine Mission ein Gut sein könne, das gestiftet wird. Somit markiert nicht allein die gegenwärtige Arbeitsweise die Organisation als Stiftung, schon gar nicht ihre juristische Rahmung, sondern ihre Ursprungsgeschichte. Somit gilt, dass

„die an den Stifterwillen gebundene Entscheidungsfindung [...] als das primäre Merkmal der Stiftung herauszustellen [ist], das die Stiftung von

Vorliegend wird der Wiederabdruck aus dem Jahr 1960 herangezogen. 
anderen Sozialkörpern unterscheidet und hinter dem andere Merkmale, etwa materielles Vermögen oder Rechtsformen, zurücktreten“" (Strachwitz 2015b: 289; vgl. auch Adloff 2004: 273; Anheier u. a. 2017a: 8).

Dies treffe bei Betrachtung der Stiftungshistorie von der Antike bis zur Gegenwart sowie über verschiedene globale Regionen hinweg gleichermaßen zu (vgl. Strachwitz 2015b: 289). Als ,gut dokumentiertes Beispiel“" nennt Strachwitz (2015b: 291) die Gründung von „Anstalten“ im 1. Jahrhundert v. Chr. in der antiken Stadt Aphrodisias (heute Südwest-Türkei, Provinz Aydın). ${ }^{6}$ Zum einen sind Stiftungen ,für die Ewigkeit gemacht" (Adam/Frey/Strachwitz 2009: V). Sie repräsentieren aber gleichzeitig den Zeitgeist ihrer Entstehungsepoche (vgl. ebd.). Aus der recht differenzierten Bestandsfestigkeit der Stiftungen, die unter Umständen mehrere Jahrhunderte alt sein können, mitunter erst vor kurzem gegründet wurden, ergibt sich ein weiterer Hinweis auf die Heterogenität des Stiftungswesens. Verstärkt um den Faktor ihrer Beständigkeit ,manifestieren sich zugleich rechtliche, wirtschaftliche, politische, religiöse, ästhetische und morphologische Aspekte“ (Adloff 2004: 281).

In dieser umfassenderen Lesart ist die Stiftung eine singuläre Anstalt zur Verwaltung von ideellem und materiellem Vermögen, wie auch immer es zustande gekommen ist, der eine Gründungsgeschichte mit klar benennbaren Gründungspersonen zu Grunde liegt und die einen auf das Gemeinwohl gerichteten Zweck erfüllen soll sowie an diesen gebunden zu bleiben hat. Den kleinsten gemeinsamen Nenner bildet das zweckgebundene Vermögen mit selbstständiger Organisationsform (vgl. Schlüter 2004: 22; Anheier u.a. 2017a: 11f., Strachwitz 2015b: 283 ff. in Anlehnung an Rassem 1960: 492 sowie ähnlich: Borgolte 2004: 23; Adloff 2010: 13; Sigmund 2004). Als ,soziale Tatsachen" (Adloff 2004: 281) vereinigen bzw. vermengen sie unterschiedliche Handlungslogiken, sind thematisch wie sphärisch breit aufgestellt und nutzen ihre spezielle Verfasstheit zu ihren Gunsten aus. Um die juristische Perspektive ergänzt, müssen Stiftungen als private, eigenständige Rechtsfigur mit Sitz und Leitungsgremium auftreten, sich also selbst verwalten, dürfen keine Gewinne an Mitglieder oder den Stifter selbst als Einkommen ausschütten und müssen sich selbst als Stiftung verstehen (zu den beiden letztgenannten Kriterien vgl. Anheier u. a. 2017a: 10). in der Antike. 


\subsubsection{Stiftungslandschaft in Deutschland}

Betrachtet man, um den historischen Zeitraum erfassbar zu halten und auch etwaige Verwerfungen der Kapital- und Sozialstrukturen durch den Zweiten Weltkrieg auszuklammern, das Jahr 1945 für Deutschland auch als eine „Stunde Null“ der Stiftungen (Strachwitz 2007: 99), zeigt sich, dass die Errichtung einer solchen Einrichtung seither äußerst beliebt wurde. Bis 2007 nahmen Neugründungen (mit einer gewissen Pause zu Zeiten der Wiedervereinigung) stetig zu. Gab es 1990 noch 181 Neugründungen von ,selbstständigen Stiftungen bürgerlichen Rechts“ (nach $\S \S 80$ ff. BGB), erreichte diese Zahl 2007 einen Spitzenwert von 1.134 Neugründungen (vgl. Bundesverband Deutscher Stiftungen 2011: 20), ging danach leicht zurück, bewegt sich aber weiterhin auf einem hohen dreistelligen Zahlenniveau jährlich. Das Stiftungsvermögen insgesamt wird auf 100 Milliarden Euro geschätzt (Stand November 2017, Bundesverband Deutscher Stiftungen). Insgesamt gibt es etwa 22.800 Stiftungen in Deutschland (Stand Mai 2018), allerdings muss hierbei genauer unterschieden werden. In der detaillierten Aufschlüsselung von 2011 sind diese näher klassifiziert. Sind etwa 96 Prozent der Stiftungen tatsächlich juristisch nach $\S 80$ BGB organisiert (wobei für ,nur' 94 Prozent eine Gemeinnützigkeit anerkannt ist) und unterliegen damit der staatlichen Stiftungsaufsicht, sind vier Prozent in anderen juristischen Formen angelegt. Dieser Umstand könnte zu vernachlässigen sein, für die Betrachtung von öffentlichkeitswirksam auftretenden Akteure ist sie aber hoch relevant. Denn insbesondere viele prominente Stiftungen haben eine andere juristische Rahmung als sie für Stiftungen im engeren Sinn juristisch vorgesehen ist: sogenannte unternehmensnahe Stiftungen werden zum Teil als (g)GmbH gegründet. ${ }^{7}$ Parteinahe Stiftungen sind fast ausschließlich als eingetragene Vereine (e.V.) organisiert (siehe Exkurs und Tabelle 1 unten), Ausnahmen: Friedrich-Naumann-Stiftung für die Freiheit, Hans-Böckler-Stiftung). Neugründungen von Stiftungen nach $§ 80$ BGB werden zu einem großen Anteil von Privatpersonen vorgenommen, die zum Beispiel ihr Privatvermögen in eine Stiftung überführen möchten. Dies traf im Zeitraum von 2001 bis 2010 auf 2.515 Neugründungen von 4.062 insgesamt zu (eigene Berechnung nach Bundesverband Deutscher Stiftungen 2011: 79). Das Kapital, das von Privatpersonen in Stiftungen angelegt wird, ist im Vergleich zu den Stiftungen, die von juristischen Personen oder von kirchlichen Einrichtungen errichtet wurden, relativ gering. Sie zählen selten zu den finanziell stärkeren Stiftungen. Denn der Bundesverband konstatiert, dass die Verteilung von Stiftungsvermögen ,sehr konzentriert [ist]: Eine Minderheit der

7 (gemeinnützige)Gesellschaft mit beschränkter Haftung; so zum Beispiel Robert-Bosch-Stiftung $\mathrm{GmbH}$ 
Stiftungen verfügt über die Mehrheit des Vermögens“ (Bundesverband Deutscher Stiftungen 2011:27). In den statistischen Werten des Bundesverbands Deutscher Stiftungen werden die Vermögenswerte nur von Bürgerstiftungen nach regionaler Verteilung genannt, die Jahreszahlen aus verschiedenen Kalenderjahren gezogen, wobei Dresden der Spitzenreiter mit ca. 13 Millionen Euro Gesamt-Stiftungsvermögen bildet (1999), mit Abstand gefolgt vom zweitstärksten Bezirk Hamburg mit ca. 9 Millionen Euro (auch 1999) (vgl. Bundesverband Deutscher Stiftungen 2011: 28). Die drängende Frage in diesem Fall, wie sich Stiftungsvermögen auf die verschiedenen Rechtsformen verteilt und die begründete Annahme, dass zum Beispiel von juristischen Personen gegründete Stiftungen über das mit Abstand größte Kapital verfügen, bleibt jedoch im öffentlich greifbaren Material offen. Hinweise liefert die Auflistung der „15 größten Stiftungen des privaten Rechts nach Vermögen“ (Bundesverband Deutscher Stiftungen 2011: 70). Wird für die Spitzenreiterin, die Robert Bosch Stiftung $\mathrm{GmbH}$, ein Wert von über 5 Millionen Euro gelistet, kommt die ebenfalls prominente Körber-Stiftung auf dem 14. Platz nur noch auf ca. ein Zehntel der Summe. Die Daten beruhen auf einer freiwilligen Selbstauskunft für das Jahr 2010, was bedeutet, dass gegebenenfalls Stiftungen oder Ausgründungen fehlen. In dieser Kategorie sind die Stiftungen, die als formrichtige Stiftungen nach $\S 80 \mathrm{ff}$. BGB gegründet wurden und die unter der staatlichen Stiftungsaufsicht stehen sowie Stiftungsorganisationen mit einer anderen privatrechtlichen Grundlage (namentlich $\mathrm{GmbH}$ ) gemischt vertreten.

Diese Ausführungen zeigen, dass es nicht ganz einfach ist, die Stiftungslandschaft in Deutschland auf einen Nenner zu bringen: selbst der Bundesverband Deutscher Stiftungen weist in seinen Statistiken bemerkenswerte Ungenauigkeiten aus, die sich durch die uneinheitliche bürokratische Erfassung ergeben oder durch die unterschiedlich langen Prozesse bei der Errichtung von Stiftungen, bis diese als Stiftung oder als gemeinnützige Organisation anerkannt sind. Die Stiftungsaufsicht ist zudem Ländersache. Die Statistiken bilden somit lediglich Tendenzen ab. Es sind Kategorien zu unterscheiden, die in Wechselwirkung vorkommen können: Die Gründenden können natürliche Personen (einzelne Privatpersonen), juristische Personen des Kirchen- oder des bürgerlichen Rechts (z. B. Unternehmen) oder juristische Personen des öffentlichen Rechts (Anstalten wie Sparkassen, Körperschaften wie Kommunen) sein. Die Stiftung kann sich in der privatrechtlichen Rahmung unterscheiden (formrichtige Stiftung nach $\S \S 80 \mathrm{ff}$. BGB, eingetragener Verein, GmbH). Sie kann als gemeinnützig anerkannt sein, weil ihr Stiftungszweck dies zulässt, was auf die Mehrzahl zutrifft, oder auch nicht (privatnützige Zwecke, zum Beispiel Familienstiftungen). Abbildung 2 zeigt die Stiftungszwecke nach gewichteten Hauptgruppen über alle Stiftungskategorien hinweg gemischt auf. 
Abbildung 2: Prozentuale Verteilung der gewichteten Hauptgruppen der Stiftungszwecke bei Stiftungserrichtungen in Dekaden seit 1951

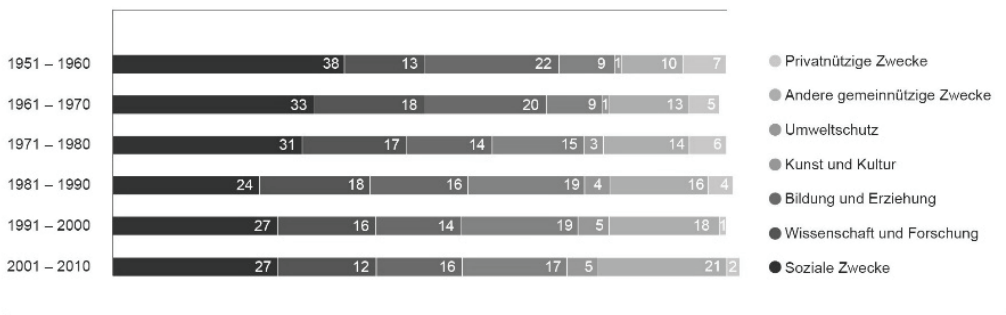

(Quelle: Eigene Abbildung nach Bundesverband Deutscher Stiftungen 2011: 36)

Es zeigt sich, dass „Soziale Zwecke“ zwar in der 1950er Jahren einen höheren Anteil der Stiftermotive ausmachten (38 Prozent), jedoch auch aktuell als häufigste Beweggründe bei einer Stiftungserrichtung angegeben werden. Der Bereich „Umweltschutz“ nahm von einem Prozent auf fünf Prozent verhältnismäßig stark zu, da vermutet werden kann, dass die öffentliche Wahrnehmung sich in diesem Punkt stark verändert hat. Auch zeigt sich deutlich, dass sich die Stiftungszwecke insgesamt relativ stark heterogenisiert haben, da die Position ,Andere gemeinnützige Zwecke“ sich von zehn auf 21 Prozent mehr als verdoppelt hat.

Abschließend soll ein Umstand benannt werden, der überraschen mag. Man könnte annehmen, Unternehmensgründung und Unternehmertum seien vor allem im letzten Jahrhundert stark männlich konnotiert gewesen und Frauen spielten zum Beispiel bei der Gründung von Bürgerstiftungen kaum eine Rolle. Die Zahlen des Bundesverbands Deutscher Stiftungen deuten jedoch an, dass sich Frauen und Männer als Stifterinnen und Stifter die Waage halten. Eine Stichprobe von 6.403 Stiftungen, die von natürlichen Personen gegründet wurden, weist 1.473 Stifterinnen und 1.517 Stifter aus. Die übrigen wurden von Frauen und Männern gemeinsam, vermutlich meist als Ehepaar, gegründet (vgl. Bundesverband Deutscher Stiftungen 2011: 85). Ob es sich bei den Stifterinnen immer um Witwen oder Töchter von Unternehmern handelt oder ob dieser relativ hohe Anteil an Stifterinnen andere Gründe hat, kann hier nicht abschließend geklärt werden. Selbst in der sogenannten „StifterStudie“ wird konstatiert, dass es ,den typischen deutschen Stifter nicht“" (Timmer 2005: 44) gebe. 


\section{Exkurs: Parteinahe Stiftungen}

Ein Typus von Stiftungen ist im Zusammenhang mit der Frage, worin noch eine Stiftung bestehe und was als anderweitige Sozietät gesehen werden muss, besonders komplex.

Tabelle 1: Entstehungsgeschichte der sogenannten „,verbände-“ bzw. „parteinahen“ Stiftungen in Deutschland auf Bundesebene bis 2017

\begin{tabular}{|c|c|c|c|}
\hline Stiftung & G.-Jahr & Beschreibung & Rechtsform \\
\hline $\begin{array}{l}\text { Konrad- } \\
\text { Adenauer } \\
\text {-Stiftung }\end{array}$ & $\begin{array}{l}1955 \\
(1964)\end{array}$ & $\begin{array}{l}\text { Bildungsstätte der Christlich Demokratischen Union } \\
\text { (CDU), } 1964 \text { Umbenennung in „Konrad-Adenauer-Stif- } \\
\text { tung" und Erweiterung der "Dienstleistungspalette”. }\end{array}$ & $\begin{array}{l}\text { einge- } \\
\text { tragener } \\
\text { Verein (e.V.) }\end{array}$ \\
\hline $\begin{array}{l}\text { Friedrich- } \\
\text { Naumann } \\
\text {-Stiftung für } \\
\text { die Freiheit }\end{array}$ & 1958 & $\begin{array}{l}\text { Gründung als „Friedrich-Naumann-Stiftung" u. a. von } \\
\text { Theodor Heuss (FDP), dem damaligen Bundespräsi- } \\
\text { denten; der Name „Theodor Heuss" wird in einer wei- } \\
\text { teren, } 1964 \text { gegründeten parteiübergreifenden Stiftung } \\
\text { weitergeführt. }\end{array}$ & $\begin{array}{l}\text { Stiftung } \\
\text { des privaten } \\
\text { Rechts }\end{array}$ \\
\hline $\begin{array}{l}\text { Heinrich- } \\
\text { Böll- } \\
\text { Stiftung }\end{array}$ & $\begin{array}{l}1988 \\
(1997)\end{array}$ & $\begin{array}{l}\text { Gründung als Stiftungsverband Regenbogen e.V., nach- } \\
\text { trägliche Anerkennung des Bundesvorstands der Partei } \\
\text { "Die Grünen“ als ihre parteinahe Stiftung und Fusion der } \\
\text { einzelnen grünen-nahen Stiftungen auf Länderebene } \\
\text { unter dem Dach der „Heinrich-Böll-Stiftung“ seit } 1997 .\end{array}$ & $\begin{array}{l}\text { einge- } \\
\text { tragener } \\
\text { Verein (e.V.) }\end{array}$ \\
\hline $\begin{array}{l}\text { Friedrich- } \\
\text { Ebert- } \\
\text { Stiftung }\end{array}$ & $\begin{array}{l}1925 \\
(1954)\end{array}$ & $\begin{array}{l}\text { Gründung zur Umsetzung des Testaments Friedrich } \\
\text { Eberts nach dessen Tod, wird zunächst durch Spenden } \\
\text { mitgetragen, seit } 1954 \text { als e.V. organisiert, sieht sich der } \\
\text { „Sozialdemokratie“ verpflichtet, tritt aber vom Corporate } \\
\text { Design unabhängig von der Partei Sozialdemokrati- } \\
\text { schen Partei Deutschlands (SPD) auf, allerdings sind } \\
\text { personenbezogene Uberschneidungen in den Verwal- } \\
\text { tungsorganen mit der SPD vorhanden. }\end{array}$ & $\begin{array}{l}\text { einge- } \\
\text { tragener } \\
\text { Verein (e.V.) }\end{array}$ \\
\hline $\begin{array}{l}\text { Rosa- } \\
\text { Luxemburg } \\
\text {-Stiftung }\end{array}$ & $\begin{array}{l}1990 \\
(2000)\end{array}$ & $\begin{array}{l}\text { Zunächst als Verein Gesellschaftsanalyse gegründet, } \\
\text { wurde } 1992 \text { durch einen Beschluss der damaligen } \\
\text { Partei des Demokratischen Sozialismus (PDS) als die } \\
\text { ihr nahestehende Stiftung anerkannt. } 2000 \text { erfolgte die } \\
\text { Umbenennung in „Rosa-Luxemburg-Stiftung - Gesell- } \\
\text { schaftsanalyse und Politische Bildung e.V... Seit der } \\
\text { Fusion von PDS mit der WASG zur Die Linke steht sie } \\
\text { politisch dieser neu entstandenen Partei nah. }\end{array}$ & $\begin{array}{l}\text { einge- } \\
\text { tragener } \\
\text { Verein (e.V.) }\end{array}$ \\
\hline $\begin{array}{l}\text { Hanns- } \\
\text { Seidel- } \\
\text { Stiftung }\end{array}$ & 1966 & $\begin{array}{l}\text { Trotz seiner Nähe zur „Bayern-Partei" Christlich-Soziale } \\
\text { Union (CSU), erhallt die Hanns-Seidel-Stiftung Mittel } \\
\text { aus dem Bundeshaushalt und ist national wie internatio- } \\
\text { nal aktiv, Namensgebung nach dem ehemaligen Minis- } \\
\text { terpräsidenten Bayerns, Hanns Seidel (1901-1961). }\end{array}$ & $\begin{array}{l}\text { einge- } \\
\text { tragener } \\
\text { Verein (e.V.) }\end{array}$ \\
\hline $\begin{array}{l}\text { Hans- } \\
\text { Böckler- } \\
\text { Stiftung }\end{array}$ & 1977 & $\begin{array}{l}\text { Gegründet durch Beschluss des Deutschen Gewerk- } \\
\text { schaftsbundes, die beiden Organisationen „Hans-Bö- } \\
\text { ckler-Gesellschaft" und „Stiftung Mitbestimmung" zur } \\
\text { „Hans-Böckler-Stiftung" zusammenzuschließen. }\end{array}$ & $\begin{array}{l}\text { Stiftung } \\
\text { des privaten } \\
\text { Rechts }\end{array}$ \\
\hline
\end{tabular}

(eigene Darstellung in Anlehnung an die Stiftungs-Homepages sowie Klaassen 2016: 35 ff.) 
Sogenannte parteinahe Stiftungen gehen zumeist auf eine eindeutige Gründungsgeschichte zurück, haben einen gemeinnützigen Zweck, einen Sitz und verfolgen neben der finanziellen Unterstützung von Personen oder Institutionen auch ideelle Werte. Zudem verstehen sie sich selbst als Stiftung. In Antworten auf die Frage nach dem Stiftungsvermögen bzw. zu ihrer Finanzierung wird es diffus. Parteinahe Stiftungen finanzieren sich fast ausschließlich durch die öffentliche Hand (vgl. Klaassen 2016: 59), das heißt, sie sind in ihrer Handlungslogik staatlichen Vorgaben stark unterworfen. Auf der anderen Seite sind sie gleichzeitig im juristischen Sinne als Stiftungen organisiert (siehe Tabelle 1). Klaassen zeigt in seiner Dissertation ausgiebig auf, welche Summen den jeweiligen Organisationen durch Bundesmittel und welche durch die Länder zugeführt werden.

Dabei stellt sich heraus, dass die häufige Kritik an einer Intransparenz dieser Entitäten durchaus berechtigt ist, speisen sich die Zuwendungen zum Beispiel der Bundesmittel nicht einfach aus einem Budget aufgrund einer gesetzlichen Grundlage, sondern werden über die Etats verschiedener Ministerien und Ausschüsse ohne klare Vorgaben verteilt (vgl. Klaassen 2016: 59 ff.). Klaassen schätzt die Zuwendungen aus Bundesmitteln an die parteinahen Stiftungen für 2010 auf 400 Millionen Euro (ebd.). Hinzu kommen Länderzuschüsse von insgesamt 10,5 Millionen Euro (vgl. Klaassen 2016: 65). Es gibt dennoch gute Gründe, die parteinahen Stiftungen in der deutschen Stiftungslandschaft einzuordnen:

Ihre (der parteinahen Stiftungen, Anm. NDS) parteiideologische Ausrichtung ist Voraussetzung für die öffentliche Finanzierung, sie müssen sich gleichzeitig jedoch parteifern gerieren, um finanzielle Zuwendungen zu erhalten (Pascher 2001: 7).

Ihre Bestrebungen, auch ideelle Werte zu vermitteln, bilden daher den Hauptanlass für die Finanzierung. Sie sind somit eine Art Zwitterwesen der Stiftungslandschaft: sie ,bewegen [...] sich in der Sphäre zwischen Staatlichkeit und Zivilgesellschaftlichkeit" (Pascher 2001: 19). Das gilt in ähnlicher Weise für unternehmensnahe Stiftungen, die ihre Haushalte mit Mittel füllen, die durch unternehmerisches Handeln gewonnen wurden. Laut Rassem (vgl. oben) macht daher der Gründungsmythos mit seinen ideellen Bestrebungen die Sozietät zur Stiftung, nicht wie in anderen gesellschaftlichen Sphären die Art der „Geldbeschaffung“. So wäre die finanzielle Existenz des Staates ohne Steuereinnahmen kaum denkbar, genauso wie der private Markt bestrebt ist, über Umsatz und hohe Gewinnmargen Geldmittel zu akkumulieren. Die Stiftung ist derweil wie jede andere Organisation auch der Einhaltung der Rechtsordnung unterworfen. 


\subsubsection{Stand der Stiftungsforschung}

Wie ausgeführt, gehören Fragestellungen zu Stiftungen zu den weniger beforschten Themen der Sozial- bzw. Politikwissenschaft. Verhältnismäßig reich ist noch die Literaturlage zu Abhandlungen darüber, wie die juristische und praktische Handhabung von Stiftungen zu erfolgen habe (zum Beispiel Pues 1999, Wachter 2001, Pues 2010, Wigand u. a. 2011, Merten 1999). Einige Schriften beschäftigen sich allein mit der Historizität von Stiftungen (zum Beispiel Campenhausen 2015, von Reden 2015). Einige Studien möchten neben einer quantitativen Deskription der Stiftungslandschaft (siehe 2.3.2) allein die ökonomische oder arbeitsmarktbezogene Bedeutung dieser Organisationen belegen. Erst in der neueren Forschung finden sich wenige Abhandlungen zu Stiftungen als gesellschaftliche, politische oder soziale Akteure in verschiedenen Spannungsfeldern.

Einen Schnittpunkt zum „Markt“ behandelt Schnurbein (2015), indem er die philanthropische Idee der Stiftungsgründung bei unternehmensnahen Stiftungen hervorhebt. Im Verlauf der jüngsten Zeit sei durch die stärker operative Ausrichtung der Stiftungen aus der "Verwaltungsanstalt Stiftung" mehr und mehr eine Managementaufgabe geworden (vgl. Schnurbein 2015: 248), die aber, anders als die klassisch manageriale Aufgabe der Gewinnmaximierung in Unternehmen, hier darin liegt, den Stiftungszweck als Sachziel zu erfüllen. Diese Werteorientierung grenze die Stiftung auch klar von anderen NonprofitOrganisationen wie Vereinen oder Verbänden ab (vgl. ebd.).

Deutlich intensiver wird die in Abgrenzung zur staatlichen Sphäre behandelt. So diskutiert Klaassen (2016) im Rahmen seiner juristischen Dissertation, wie oben erwähnt, wie das Konzept der ,parteinahen Stiftung“ zu verstehen ist bzw. welche Ambivalenzen mit ihm verbunden sind. Pascher beschäftigt sich in ihrer Dissertation (2001) ebenfalls mit parteinahen Stiftungen, allerdings vor dem Hintergrund der internationalen Aktivitäten dieser Organisationen fokussiert auf ihre Tätigkeit im Globalisierungs-Kontext. Die Dissertation von Striebig (2017) befasst sich mit der „Legitimierung von Stiftungen“ vor dem Hintergrund des in der Politikwissenschaft vielfach diskutierten „Demokratiedefizits" von öffentlich auftretenden, demokratisch nicht legitimierten Akteuren. Dabei bezieht er die Diskussion um accountability der NPOs auf die Rechenschafts- und Stakeholderstrukturen vor allem von Stiftungen im juristisch engeren Sinn. Damit wendet er eine eher enge Definition von Stiftungen an und schließt zudem parteinahe Stiftungen gänzlich aus (vgl. Striebing 2017: 14).

Der Frage nach der Legitimation geht mit einer sehr gründlichen Recherche zur Genese, Historizität und (staats-) philosophischen Sichtweise auf Stiftungen auch Rupert Strachwitz in seinen Schriften aus den Jahren 2015 (,Stiftun- 
gen und moderner Staat. Zur Genese und Argumentation einer Legitimitätsdebatte”) und 2016 („Die Stiftung - ein Paradoxon?”) nach. Die Frage nach der Legitimation drängt sich bei Stiftungen stark auf.

Die weitergehende Frage, ob und in welchem Maße Stiftungen mit ei-
ner modernen demokratischen Gesellschaft kompatibel bzw. in einer
solchen tolerabel sind, wird kaum gestellt, obwohl eine sich dem demo-
kratischen Willensbildungsprozess ausdrücklich nicht unterwerfende,
zugleich aber jedenfalls in ihrer überwältigenden Mehrheit subjektiv
dem Allgemeinwohl verpflichtete Organisation [...] doch eigentlich
von vornherein als demokratie-unverträglich erscheinen müsste, es sei
denn, es ließen sich andere stichhaltige Begründungen für das Gegenteil
finden (Strachwitz 2015b: 286).

Diese stichhaltigen Begründungen versucht Strachwitz zu finden. Das „Schenkungsprinzip“ (Strachwitz 2015b: 306) macht aus der Stiftung einen Akteur der Zivilgesellschaft, da es das „Paradigma der drei Arenen mitbegründet“ (Strachwitz 2015b: 307). Die Frage nach der Kompatibilität mit der nationalstaatlich organisierten Demokratie löst er dergestalt, dass er aus einem libertären Verständnis von Gesellschaftsordnung heraus konstatiert, dass „Verfassungskompatibilität [...] demnach auf Gesetzestreue, nicht auf die prinzipielle Kompatibilität der inneren Ordnung" (Strachwitz 2015b: 307) zu gründen sei. Als weiteres Argument führt er die Handlungsmaxime von Stiftungen an, der zufolge sie sich aufgrund ihrer spezifischen Genese anders als politische oder andere zivilgesellschaftliche Akteure um Nachhaltigkeit bemühen, womit zwar auf der einen Seite die Kritik über die „Herrschaft der ,toten Hand““ (Strachwitz 2015b: 308) aufrechterhalten wird. Andererseits wird aber die Interventionskraft dieser ,toten Hand“ gegenüber einer Politik, die der Kritik ausgesetzt ist, zu Lasten zukünftiger Generationen kurzatmige, visionslose Ziele zu verfolgen ,geradezu herbeigesehnt“ (Strachwitz 2015b: 308). Diese Argumente können indes zunächst nur als Indizien gewertet werden, schlussfolgert Strachwitz doch selbst, dass ,,sie theoretisch zu kurz" griffen (Strachwitz 2015b: 310). In seinem ein Jahr später erschienenen Buch „Die Stiftung - ein Paradoxon?“" verdichtete Strachwitz diese Ansätze als „Elemente einer neuen Stiftungstheorie" (Strachwitz 2016: 203 ff.). Vier der bei Strachwitz (2016: 204 ff.) erwähnten Indizien für eine Stiftungstheorie sollen an dieser Stelle näher ausgeführt werden, weil diese auf eine gesellschaftliche und weniger eine juristische oder wirtschaftliche Bedeutung von Stiftungen abzielen. Sie sollen im Rahmen der vorliegenden empirischen Daten zur Handlungslogik operativer Stiftungen in Kapitel 4 näher betrachtet werden. 
Der erste Aspekt betrifft die „Komplementarität“, bei diesem Indiz geht es um das reziproke Verhältnis zwischen Stiftungen und Staat. Dabei sollen Stiftungen als Unterstützer des Staates behandelt werden, weil, wie oben erwähnt, letztere wegen ,leerer Staatskassen“ gesellschaftlichen Transformationen und diesen hieraus resultierenden Herausforderungen der ,gesellschaftlichen Heterogenität" (Strachwitz 2016: 204) nicht mehr alleine entgegentreten können.

Der zweite Punkt kann unter ,Tradition“ zusammengefasst werden, da der Nachhaltigkeitsaspekt von Stiftungen auch die Fähigkeit zur Wahrung zentraler gesellschaftlicher Werte suggeriert. Allerdings wird kritisiert, dass im Umkehrschluss überkommene Strukturen, so auch ,die Reproduktion von Eliten und damit von sozialen Ungleichheiten" (Strachwitz 2016: 205) ermöglicht werden können.

Als dritter Punkt wird die Innovationsleistung von Stiftungen genannt. Strachwitz kritisiert direkt, dass die Selbstdarstellung vieler Stiftungen, sie seien innovativ, kaum überprüft oder nachgewiesen werden könne (vgl. Strachwitz 2016: 205). Um eine Einordnung dieses Aspekts vornehmen zu können, wäre zu klären, was es mit dem sperrigen Begriff der „Innovation“ auf sich haben soll. Die Assoziation, mit Innovation sei einfach technischer Fortschritt gemeint, greife für soziale Neuerungen viel zu kurz. So belegen Forschungen seit den 1990er Jahren, ,dass man es bei Innovation mit einem komplexen sozialen Prozess zu tun hat, in dem netzwerkförmiges Zusammenwirken der vielen am Innovationsprozess Beteiligten die zentrale Rolle spielt“ (Howaldt/Schwarz 2010: 16). Somit richtet sich der Blick der Stiftungsforschung auf die Netzwerkbildung bzw. die Erzeugung von sozialem Kapital durch Stiftungsarbeit. Blättel-Mink erweitert hier das Gelingensmerkmal von Innovation einer Organisation auf die Fähigkeit ,widersprüchliche und gegensätzliche Anforderungen, die sich aus dynamischen Umwelten ergeben, anzupassen“ (Blättel-Mink/Menez 2015: 203). Also geht es letztlich um die Fähigkeit, sozial komplexe, möglicherweise konfliktgeladene Wechselbeziehungen zwischen Akteuren in gewinnbringende zu überführen.

Als vierter und letzter Punkt soll ein Komplex angeführt werden, der unter „Betreuung von nicht mehrheitsfähigen Ansätzen, Projekten und Themen“ subsummiert wird. Unter dem Aspekt des Minderheitenschutzes, einem hohen Gut in demokratisch verfassten Gesellschaften, sei dieser zu begrüßen, zugleich betont Strachwitz die zwingende Vorgabe der Gesetzestreue und die möglichen Grenzen des Minoritären.

Er gibt zu, dass viele dieser Indizien, die zum Teil bereits in der Ausarbeitung von 2015 behandelt werden, ,spekulativ bleiben“"(Strachwitz 2016: 204), da eine wissenschaftliche empirische Fundierung fehle. Dennoch werden seine Ausführungen von Forscherinnen und Forschern in der umfangreichen Systematisierung der Stiftungslandschaft aufgegriffen und fortentwickelt und in ein 
geometrisches Gebilde überführt (vgl. Anheier u. a. 2017a). Es wird ein „Stiftungsdreieck" vorgestellt, in dem ,Ziel, Ansatz und Tätigkeitsmuster" (Anheier u. a. 2017a: 21) sortiert werden sollen, um die Kombinatorik im Stiftungshandeln hinsichtlich einer „sozialen Wirkung“ (ebd.) zu untersuchen. Hier werden die Aspekte von Strachwitz (2016) eingearbeitet. Die Zielsetzungen werden am Ende auf Grundlage der in quantitativen Erhebungen gewonnenen Daten zu drei Endpunkten unter den Titeln „Fürsorge“, „Bewahrung“ und „Wandel“" verdichtet (vgl. Anheier u. a. 2017a: 215 ff.). Diese werden für eigenständige Themenfelder (Bildung, Kunst, Soziales uvm.) separat betrachtet. Dieses Vorgehen ermöglicht eine Sortierung des sehr heterogenen Stiftungswesens in Deutschland, von dessen Entitäten bekanntermaßen die wenigsten über sehr große Vermögen oder professionelle Strukturen verfügen. Ein weiterer Hinweis, der für die Einordnung von Stiftungen in den gesellschaftlichen Kontext bedeutsam ist, ist der Bekanntheitsgrad von Stiftungen in weiten Teilen der Bevölkerung. Mit Verweis auf die Zahlen aus der „StifterStudie“ (vgl. Timmer 2005), konstatiert Meyer, dass Stiftungen in dieser Hinsicht ein „Schattendasein“ (Meyer 2015: 14) fristeten. Im Vergleich zu anderen Ländern kämen in Deutschland nur wenige Menschen im Laufe ihres Lebens mit Stiftungen in Kontakt. Neben den vielen Argumenten für die Existenzberechtigung von Stiftungen zeigt sich hier die elitäre Verfasstheit von Stiftungen und ihre Agitation in bestimmten gesellschaftlichen Kreisen.

Während für Stiftungen eine eigene Handlungslogik konstatiert wird, die sich von den Sphären „Markt" und „Staat" unterscheidet, führen Then und Kehl (2015: 262) die Idee ins Feld, bei Stiftungen handele es sich um ein Phänomen der „Hybridisierung“ der Handlungslogiken, wie sie auch in anderen Organisationen beobachtet werden könne. Auch hier fehlt indes die empirische Fundierung.

\subsection{Stiftungen als Organisationen der Zivilgesellschaft}

Der Dritte-Sektor-Ansatz (Zimmer 2000a) verortet neben Markt, Staat und Gemeinschaft ein Handlungsfeld, eine Dritte Kraft (Strachwitz 1998), die sich solidarisch organisiert und auf Grundlage individueller Entscheidungen bildet. Dieser Dritte Sektor wurde im Laufe des Kapitels als Handlungsfeld umrissen. Erweiternd zu den Ausführungen wurde der Begriff der Zivilgesellschaft als definitorisch schillernd diskutiert. Es wurde konstatiert, dass die zivilgesellschaftliche Sphäre neben der solidarischen und individuellen Komponente des Dritten Sektors zusätzlich den Anspruch der Stabilisierung der Gemeinschaft 
erfüllen sollte. Dies ist insofern eine Gratwanderung gegenüber Formen staatlicher Herrschaft, als es zivilgesellschaftlichen Akteuren um die Balance zwischen Wahrung bestimmter gesellschaftlicher Strukturen wie auch der Unterstützung von Innovationen, sozialem Wandel und der Stärkung von Bürgerrechten gehen muss. Relativ unbestritten ist der Umstand, dass es für die Konsolidierung einer starken Demokratie ,,a free and lively civil society“ (Keane 1998: 48) braucht. Zu diesem Ergebnis kommt auch Putnam in seiner bekannten Sozialkapitalstudie zur Zivilgesellschaft (1994). Wurde Zivilgesellschaft in den dokumentierten Anfängen der Antike als Herrschaft des Bürgers verstanden, bildete sich im Zuge der Jahrhunderte eine Dichotomie von herrschendem Staat gegenüber mündigen Bürgern heraus, die in einem apolitischen, aber legalen Raum Interessen anführen und Lösungen für allgemeine Probleme suchen. Somit sind Stiftungen zwingend als Teil der Zivilgesellschaft inklusive Bürgergesellschaft zu sehen, wobei es Unterschiede zwischen den Stiftungstypen gibt. Allgemein ist es nicht Aufgabe der Zivilgesellschaft, staatliche Herrschaft zu reproduzieren, aber auch, sie nicht in Frage zu stellen. Dieses Vakuum gibt viel Raum für unterschiedliche Reziprozitäten, nicht zuletzt bei Stiftungen.

Das reziproke Verhältnis zum Staat wird durch die verschiedenen Stiftungstypen laut der Literaturlage unterschiedlich bewältigt:

- Parteinahe Stiftungen werden durch die Mittelvergabe der öffentlichen Hand überhaupt erst befähigt, eigene Ziele zu verfolgen. Die Bedingungen der Mittelvergabe sind aber daran geknüpft, dass sie als zivilgesellschaftlicher Akteur auftreten, den ursprünglichen Stiftungszweck erfüllen und ideelle Werte vertreten. Hierzu sind noch erhebliche Forschungsanstrengungen vonnöten. „Bisher ist es nicht möglich von einem expliziten Forschungszweig der, Parteistiftungsforschung' zu sprechen analog etwa einer Tradition der ,Parteienforschung “" (Pascher 2001: 14).

- Unternehmensnahe und andere private Stiftungen (so auch Bürgerstiftungen) generieren ihre Geldmittel selbstständig über den Markt, sind aber der Rechtsordnung unterworfen. Durch die föderalen Strukturen und die Informationshoheit des Staates sind sie gezwungen, Kooperationen mit öffentlichen Akteuren einzugehen, um ihre gemeinwohlorientierten Ziele zu erreichen, wenn zum Beispiel Bedarfe in der Kommune ermittelt werden müssen.

- Kommunale Stiftungen verwalten privates Geld aus Bürgerstiftungen treuhänderisch, wenn dies zum Beispiel keine Nachkommen mehr tun können. Sie sind dem Stifterwillen unterworfen, genau bestimmte Ziele zu verfolgen und nachhaltig zu wirtschaften. 
Stiftungen werden in erster Linie durch eine „Schenkungsgeschichte“ im materiellen wie ideellen Sinne definiert. Obwohl Stiftungen nur etwa zwei Prozent aller Nonprofit-Organisationen ausmachen (vgl. Anheier 2003: 49), setzen die zahllosen Stiftungen mit ihren zum kleinen Teil milliardenschweren Budgets entscheidende Akzente bei der Mitgestaltung gesellschaftlicher Prozesse. Daraus resultiert die „Ergänzungsfunktion“ (vgl. Anheier 2003: 51), die Unterstützung von Projekten oder Personen, die durch öffentlich-politische Regelungen zu kurz kommen. Die Gründungsfiguren können natürliche oder juristische Personen sein, müssen aber die Sozietät in eine juristische Hülle überführen, die nachhaltig ,dem höchst individuell [...] konnotierten“ (Zimmer 2005: 15) Willen der Stiftenden unterworfen ist. Die Stiftungstypen unterscheiden sich im Spannungsfeld zwischen Markt, Staat und Gemeinschaft in Nähe bzw. Ferne. So können die politischen bzw. partei- und verbandsnahen Stiftungen eine größere Nähe zur demokratisch legitimierten Politik, also zu staatlichen Akteuren verzeichnen. Die unternehmensnahen Stiftungen rücken näher an den „Markt“, kommunale Stiftungen und Bürgerstiftungen gruppieren sich eher Richtung Gemeinschaft bzw. organisierte Bürgerschaft. Das Stiftungswesen insgesamt bleibt dabei der zivilen Sphäre angehörig.

Durch die Fundierung der Stiftung als Organisation, die aus der Solidarität mit dem Gemeinwohl von einem sich als mündig, wirtschaftlich stark verstehenden Bürger gebildet wird, wird nur eine Seite der Medaille betrachtet. Diese Ambitionen treten bei der Stiftung gepaart mit dem individuellen Wunsch nach Ansehen, Andenken und nachhaltiger Selbstwirksamkeit auf und sind daher gleichzeitig individualistisch. Dabei sind Stiftungen in der Bevölkerung eher selten bekannt. Im Zuge der bisher vorgestellten Erkenntnisse muss beim Stiftungswesen von einem Elitenprojekt gesprochen werden, da die Fähigkeit, eine Stiftung zu gründen, einem verhältnismäßig kleinen gesellschaftlichen Kreis vorbehalten ist. Die juristische Unabhängigkeit stützt die Prämisse, dass Stiftungen fernab vom Marktgeschehen oder staatlichen Handlungslogiken agieren können. Inwiefern es Interdependenzen zwischen ihnen und in erster Linie staatlichen Institutionen gibt, kann wahrscheinlich nur für jeden Einzelfall geklärt werden, wobei die personelle und strukturelle Verwobenheit untersucht werden müsste. Dass Stiftungen nicht nur fördernd, sondern auch operativ tätig sind und dabei individuelle wie gemeinwohlorientierte Motive miteinander verbinden, macht sie nach Zimmer (2005) gegenüber einem „schwachen Sozialstaat" zu einer perfekten Organisation der Zeit, vor dem Hintergrund der Globalisierung, gesellschaftlicher Heterogenität und Individualisierung. Die Vermögenswerte sind von der Gesamtsumme sind mit 100 Milliarden insgesamt nicht vernachlässigenswert, ${ }^{8}$ wenngleich, verteilt auf über 20.000 Stiftungen,

8 Vergleich: Die Ausgaben des Bundesministeriums für Arbeit und Soziales werden für 2017 mit ca. 137 Milliarden angegeben (Bundeshaushalt-Info.de, Abruf 4. Dezember 2017). 
mit einer Bündelung der Finanzmittel auf einige Dutzend großer Stiftungen allerdings die Durchschnittsstiftung nicht üppig ausgestattet ist. Der Median der Vermögenswerte lag im Jahr 2011 bei 293.000 Euro (vgl. Bundesverband 2011: 31). Allein große Stiftungen mit unternehmensähnlichen Strukturen sind nennenswerte ,Player" im gesellschaftlichen Kontext, da sie sich im Laufe der Zeit das Handlungsrepertoire privatwirtschaftlicher Unternehmungen angeeignet haben.

Auf Grundlage der vorliegenden Erkenntnisse muss der gesellschaftliche Standort des Stiftungswesens in Deutschland wie folgt bezeichnet werden: Stiftungen sind Organisationen der Zivilgesellschaft, da sie auf Anstrengungen von Individuen zurückgehen, eine Sozietät zu gründen, die eine soziale Wirkung hat. Dabei erfüllen die Stiftungen je nach Typ unterschiedliche Rollen, die mal mehr, mal weniger das demokratische Defizit herausfordern. Eine detaillierte sozialwissenschaftliche Stiftungstheorie steht im Übrigen aus. Die empirischen Daten sollen dahingehend abgeglichen werden, ob Stiftungen selbst Anhaltspunkte für eine Stiftungstheorie liefern können bzw. wie sie das Demokratiedefizit aus der Innenperspektive bewerten.

Es deutet alles darauf hin, dass bei der Diskussion um die Existenzberechtigung von Stiftungen drei Elemente zentral sind: Das Schenkungsprinzip, das Stiftungen zu Akteuren der Zivilgesellschaft erhebt, die Gesetzestreue, der zufolge Stiftungen als legale Organisation im Rahmen der staatlichen Rechtsordnung tätig sind, sowie die Operationslogik der Nachhaltigkeit, nach der die (politischen) Handlungen von Stiftungen als legitimiert gelten, für die sie im demokratischen Rechtsstaat theoretisch nicht zuständig sind. Sie werden legitimiert aufgrund der aktuellen Komplexität der gesellschaftlichen Herausforderungen und dem Umstand, dass politisch legitimierte Akteure nach Feststellungen der Zivilgesellschaftsforschung diese immer seltener bewältigen können. Bei dem Versuch, das Stiftungswesen in seiner Struktur systematisch zu erfassen, finden sich drei zentrale Dichotomien, die in der Praxis allerdings jeweils als Kontinuum zu begreifen sind: Handelt die Stiftung fördernd oder operativ? Ist es eine ,normale', kleine Stiftung, die eng mit einer philanthropischen Idee des Stifters/Stifterpaares bzw. der Stifterin verbunden ist oder hat man es mit einer größeren Organisation mit unternehmerischen Strukturen zu tun? Erwirtschaftet die Stiftung ihr Vermögen selbst oder erhält sie bedeutsame Zuwendungen aus der öffentlichen Hand?

Bedeutend schwieriger wird es in der Frage, wie die Handlungsfelder und Zielrichtungen zu systematisieren sind, da gerade große Stiftungen durch den Einsatz von Personal und monetären Ressourcen verschiedene Ziele gleichzeitig verfolgen können. Für die vorliegende Studie zur Herstellungspraxis sozialer Differenz, die ohnehin nur operativ tätige große Stiftungen betrachtet, stel- 
len sich zentral die folgenden Fragen: Erwähnen die Vertreterinnen und Vertreter der Stiftungen die Handlungen allein als Fürsorgetätigkeit oder zum Zwecke der eigenen Profilierung (Charity vs. Agenda)? Deuten sie ein komplementäres Verhältnis zum Staat an oder ein kooperatives? Verwenden sie eigene Handlungsstrategien oder bedienen sie sich am Repertoire anderer Organisationen? Schließlich ist zu fragen: Wie schätzen sie ihre Bekanntheit und Reichweite in der breiten Bevölkerung ein? Erwähnen sie soziale Gruppen explizit oder implizit und mit welchen Konnotationen?

Auf zwei Differenzgruppen wird mehr oder weniger in diesem oder einem „Nachbarrahmen“ (zum Beispiel dem freiwilligen Bürgerengagement) der Zivilgesellschaftsforschung etwas eingegangen: Einerseits wird vordergründig die Wirkkraft der Zivilgesellschaft als emanzipatorisches Feld für Frauen behandelt, einschließlich der Frage, wie diese in dem Zivilgesellschaftsdiskurs zu verorten sind. Hierzu konstatiert Gabriele Wilde (2014: 210), dass Zivilgesellschaft ein Bereich sei, der ,durch Geschlechterverhältnisse als Macht- und Herrschaftsverhältnisse durchzogen ist" (ebd.). Bei der Betrachtung der Kategorie „Ethnizität“ zeigen sich ähnliche Befunde. Hierzulande beschränkte sich beispielsweise die Forschung auf der Mikro-Ebene des Bürgerengagements bei (Nachfahren von) Migrierten vornehmlich auf die Frage, was ,, die Aufnahmegesellschaft davon hat", wenn sich Migranten bürgerschaftlich engagieren“ (Dağlar-Sezer 2013: 149) und weniger auf andere mögliche Deutungen (zum Beispiel die emanzipatorische Wirkung dessen) oder gar die Potenziale einer solchen Anstrengung. Der Paradigmenwechsel, wie Menschen ohne deutsche Familiengeschichte in der Zivilgesellschaft zu lokalisieren sind, verbleibt neben puzzlehaft vorgetragenen Perspektiven (zum Beispiel im Sammelband „Engagierte Migranten: Teilhabe in der Bürgergesellschaft“, 2016) indes eher in der Dritte-Sektor-Tradition der deskriptiven quantitativen Erhebung zu Zahlen, wie, in welchem Umfang und mit welchen Funktionen bestimmte Gruppen in bestimmten Regionen Deutschlands aktiv sind oder eben nicht. ${ }^{9}$

Unabsichtlich referiert Wilde eine Anschlussfähigkeit an qualitative empirische Forschung, wenn sie die Zivilgesellschaft als Sphäre beschreibt, die, wie jede andere soziale Praktik, in ihrem Wirken letztlich auf ,substantielle Wertvorstellungen" (Wilde 2010: 220) zurückgreift und diese repräsentiert. Wilde schreibt weiter: die „Untersuchungsperspektive [der Abhandlung Wildes,

9 Beispiele hierfür sind von Halm/Sauer die Studie (2004),„Freiwilliges Engagement von Türkinnen und Türken in Deutschland“; Gensicke/Picot/Weiß (2006), ,Freiwilliges Engagement in Deutschland 1999-2004“ (Sonderstichprobe Migranten); Vilain/Dağlar (2005) „Integration von Migranten durch bürgerschaftliches Engagement“; Dağlar/Wolf (2008) „Bürgerschaftliches Engagement und Integration von Migrantinnen und Migranten im Kleingartenwesen“; Sezer/Dağlar (2009) ,TASD-Spektrum 2: Die Identifikation der TASD mit Deutschland“. 
Anm. NDS] richtet sich [...] darauf, wie Realität, also kollektive und geschlechtliche Identitäten, Institutionen, Strukturen und Handlungen in Form bestimmter Bedeutungen erzeugt werden“" (ebd.). Dies wird auch in der vorliegenden Studie zum Anlass genommen, genauer zu betrachten, wie Stiftungen „substantielle Wertvorstellungen“ (ebd.) transportieren. Daher wird im folgenden Kapitel die Konzeption und der aktuelle Forschungsstand für „Humandifferenzierungen“ (Hirschauer 2017) für das vorliegende Forschungsvorhaben aufgezeigt. 


\section{Soziale Differenz}

\subsection{Herausforderungen einer Konzeption soziale Differenz}

\subsubsection{Ausgangssituation}

Wie in der Einleitung (Kapitel 1) dargestellt, ist es für die vorliegende Studie erforderlich, eine Forschungsperspektive bzw. einen Analyserahmen zu skizzieren, um den Umgang mit sozialer Differenz eines organisationalen zivilgesellschaftlichen Akteurs analysieren zu können. Hierzu soll eine synoptische Darstellung einschlägiger Ansätze zu einem Forschungsrahmen zusammengeführt werden, um die Vagheit des Begriffs ,soziale Differenz' für das vorliegende Forschungsvorhaben greifbar zu machen. Dieses Vorgehen hat sich bereits in zwei anderen Studien bewährt, die auf ein poststrukturalistisches Verständnis von Differenzierung zurückgreifen. Ähnlich wie hier widmet sich Sonja Kubisch (2008) der Frage, wie in der Arbeit der freien Wohlfahrtspflege soziale Differenz habituell erzeugt wird. Getreu dem konstruktivistischen Paradigma geht sie davon aus, dass soziale Differenz per se nicht existiert (vgl. Kubisch 2008: 13). Es wird, so die Annahme, ,in der Interaktion von den verschiedenen Beteiligten hergestellt" (ebd.). In einem anderen Beispiel für eine Studie zur Erarbeitung einer Forschungsperspektive zu sozialer Differenz definiert Claudia Machold mit einem ethnographischen Ansatz unter dem Titel „Kinder und Differenz" diese mit dem Hinweis wie folgt:

„Mit sozialer Differenz sind solche Differenzen gemeint, die für die ,Seinsordnungen' in der gegenwärtigen Gesellschaft insofern relevant sind, als sie sowohl den Zugang zu Ressourcen regulieren als auch bestimmen, als wer jemand wie leben muss, um anerkennbar zu sein.“ (Machold 2015: 18, Hervorhebung im Original)

Bedenkt man nun die thematische und zielgruppenspezifische Vielfalt von Stiftungsprojekten, die es an dieser Stelle zu untersuchen gilt, wird deutlich, warum diese Vielfalt an Themen und Handlungsfeldern nicht ohne weiteres mit einem einzigen theoretischen Zugang erfasst werden kann. Machold deutet bereits an, dass es bei sozialer Differenz neben der Komponente der Ressourcenverteilung eine weitere gibt, nämlich die der normativen Vorstellung von Zugehörigkeiten und Zuschreibungen. Dieser Zweiklang lässt sich ähnlich in der Skizze zur gesellschaftlichen Standortbestimmung von Stiftungen in Kapi- 
tel 2 erkennen, bei der neben der „Ergänzungsfunktion“ (Anheier) zu staatlichen Fürsorgeleistungen auch die der politischen Akteurin zu kommt, die Bürgerkompetenzen stärkt und dem Staat gegenüber mit einer bestimmten Vorstellung von Gesellschaft als demokratisch-pluralistisches Gegengewicht auftritt.

In Kapitel 3 werden daher unterschiedliche theoretische Ansätze vorgestellt, die die Hervorbringung sozialer Differenz thematisieren und ihre Relevanz für die vorliegende Fragestellung diskutiert. Doch vorab ist zu klären, welche Überlegungen dazu geführt haben, ausgerechnet die vorgestellten Ansätze auszuwählen. Da unmöglich zunächst alle bestehenden Theorien der Soziologie bzw. der Sozialwissenschaft zu sozialer Differenz erarbeitet werden können, die sich von nah und fern mit sozialer Differenzierung beschäftigen, um im Nachgang eine Sortierung vorzunehmen, soll der Forschungsgegenstand als Filter dienen.

Bereits erwähnt wurde das konstruktivistische Paradigma, das als Prämisse der Fragestellung zum Umgang bzw. zur Herstellungsweise sozialer Differenz zugrunde liegt. Daher sind solche Konzepte als relevant einzuschätzen, die soziale Differenz grundlegend als gesellschaftliche Konstruktion betrachten. Allgemein trifft das auf die Grundidee der Soziologie als Disziplin im weitesten Sinne zu, allerdings behandeln spezielle theoretische Ansätze diese Sicht im engeren Sinne. Diese Annahme richtet sich dabei nicht nur auf gesellschaftliche Verhältnisse (Makro-), sondern auch auf Interaktionen (Mikroebene). Stiftungen wirken mutmaßlich auf all diesen Ebenen.

Die Fragestellung handelt zudem davon, dass seitens der Stiftungen $\mathrm{Zu}-$ schreibungen zu sozialen Merkmalen von Personen(gruppen) vorgenommen werden, da sie als soziale Akteure operativ arbeiten. Für die Formulierung eines Forschungsrahmens werden demnach die Hinweise aus theoretischen Ansätzen gebraucht, die sich mit der Benennung von sozialer Differenz befassen.

Bei der Intervention in gesellschaftliche Verhältnisse üben Stiftungen als organisationale Akteure Kritik an bestehenden Zuständen aus. Dieser Punkt soll in Weiterführung zu der Analyse der Benennungspraxis behandelt werden. Interessant wird die Beantwortung der Frage, inwiefern Essentialisierung, also die Wesenszuschreibung auf wenige, unveränderbare Merkmale auf ganze Gruppen thematisiert wird. Dies kann als Voraussetzung, Problem oder Hindernis für die Perspektivierung von sozialer Differenz eingebracht werden.

Stiftungen nehmen sich dem Selbstverständnis nach vor, unerwünschte gesellschaftliche Verhältnisse abzumildern bzw. zu beenden. $\mathrm{Zu}$ diesen gehören gesellschaftliche Verhältnisse, die mit dem Ausdruck ,soziale Ungleichheit ${ }^{\star}$ versehen werden. Ihre Budgets sind nicht im Entferntesten mit jenen staatlicher Fördermaßnahmen vergleichbar (siehe Kapitel 2). Es ist von kleinen Impulsen auszugehen, die sie bewerkstelligen können, indem sie Differenzmerkmale wie 
Geschlecht, Ethnizität oder Klassenlage, die jeweils soziale Ungleichheit oder Differenz markieren, gleichzeitig in den Blick nehmen. Dadurch können Stiftungen ihre Zielgruppen stärker eingrenzen. Daher ist ebenfalls zu betrachten, welche theoretischen Ansätze eine Anzahl von Ungleichheitsmerkmalen zugleich zusammenführen.

Abschließend ist zu überlegen, wie der Umgang mit sozialer Differenz aus einer Meso-Perspektive bewertet werden kann, da Stiftungen mit dem Fokus auf soziale Gruppen eine bestimmte Haltung gegenüber sozialer Differenz einnehmen.

All das zu berücksichtigen, ist eine Herausforderung. In erster Linie besteht diese darin, dass es ,auf der Baustelle der Gesellschaftstheorie“ (Knapp 2008: 150) eine „folgenreiche [...] Trennlinie“ (ebd.) zu verzeichnen gilt, nämlich die der Spaltung der Theorien zu sozialer Ungleichheit und der Differenzforschung (vgl. Knapp 2008: 151 f.), die die Verschränkungskonzepte sozialer Differenzmerkmale besonders hart trifft. Das gilt vor allem für die deutschsprachige Tradition (vgl. ebd.). Der Zweiklang, soziale Differenz sei zum einen die Analyse der Verteilung von Ressourcen (soziale Ungleichheitsforschung) und die der Zuschreibung von Merkmalen (Differenzforschung), finden sich in der sozialwissenschaftlichen Theorientradition eher als Nebeneinander. Dieser Umstand wird aus einer sozialstrukturanalytischen Sicht unter anderem durch Autorinnen und Autoren des Sammelbands, herausgegeben von Klinger u. a., illustriert, beispielsweise im Aufsatz „Komplexe Ungleichheitsverhältnisse: Klasse, Ethnie und Geschlecht" (2007). Schwinn beschreibt hier, wie zum Beispiel die sehr unterschiedlichen Forschungstraditionen zu „Klasse/Schicht“ im Rahmen der sozialen Ungleichheitsforschung kaum mit den Schieflagen in Einklang zu bringen sind, die durch askriptive Merkmale wie Geschlecht oder Ethnizität hervorgerufen werden. Ungleichheitsrelevante Differenzmerkmale wie Geschlecht, Ethnie und Klasse werden auf Handlungsfelder (Arbeitsmarkt, Bildungssystem, Einkommen; ,Arenen' vgl. Weischer 2011) fokussierend analysiert. Sie spielen allesamt eine große Rolle (vgl. Schwinn 2007: 273), die Erklärungsmuster sind allerdings selten identisch. So ist der Bezug von „Geschlecht“" auf Erwerbstätigkeit eine völlig andere als der von „Ethnizität"“ auf das Bildungssystem.

Weiter abstrahiert wird dieser Gedanke, wenn man bedenkt, dass die „Klassenbasis [...] über quantifizierbare materielle Ressourcen, Geschlecht über eine dual-exklusive und Ethnie über eine zwei- bis mehrpolige kategoriale $\mathrm{Zu}$ gehörigkeit bestimmt“" wird (Schwinn 2007: 276). Diese Beobachtung wird durch Hirschauer fortgeführt, indem er formuliert, dass bei der Betrachtung sozialer Unterschiede die „für sich selbst konstitutive Leitdifferenz" (Hirschauer 2014: 180) selten übergangen werden könne. Mitunter werden solche the- 
oretischen Lücken in den gesellschaftswissenschaftlichen Disziplinen durchaus diskutiert. So formuliert Andreas Wimmer den Anspruch an eine Konzeption sozialer Differenz, die diese Lücken schließen soll:

First it should be comprehensive and offer an adequate type for all known empirical cases. A good typology also exhausts the range of logical possibilities. Furthermore it is coherent: all types or taxa are created with reference to the same fundamentum divisionis and the various fundamenta used on different levels of a taxonomy are of the same nature. Finally, a typology should be heuristically fruitful and advance theoretical and empirical reasoning in a particular domain [...] (Wimmer 2008: 1029, Hervorhebungen im Original).

Neben der Überwindung der „Theorienspaltung“ in Ungleichheits- und Differenzforschung gilt es im vorliegenden Fall weiteren Ansprüchen zu genügen: Soziale Differenzkategorien wirken nicht nur gleichzeitig, sondern möglicherweise wechselseitig.

\subsubsection{Vorgehensweise}

Der Herausforderung, die mit den Ansprüchen an die Formulierung eines Forschungsrahmens vor dem Hintergrund der erläuterten Theorienspaltung (vgl. Knapp 2008) einhergeht, gilt es sich nun in diesem Kapitel explizit zu stellen. Die Annahme geht dahin, dass eine essentialistische Herangehensweise eine Weiterentwicklung bestehender Theorien zu sozialer Differenz von vorneherein verschließen muss. Schwierig wird die Überwindung allerdings, weil konkrete Differenzkategorien wie Geschlecht, Ethnizität und Klasse auf jeweils eigens ausgerichtete Forschungstraditionen (Geschlechterforschung, Migrationsforschung, soziale Ungleichheitsforschung) zurückgehen und auf je eigenen gesellschaftspolitisch-historischen Ausgangslagen (vgl. Klinger/Knapp 2008) beruhen, in deren Gestalt sie mehr oder weniger eine Essentialisierung erfahren (müssen), um an „Analysefähigkeit“ nicht durch „Beliebigkeit“" einzubüßen. Zum anderen unterliegen beispielhaft diese drei großen „Achsen der Ungleichheit" (Klinger u. a. 2007) wie oben bereits angedeutet, völlig unterschiedlichen Herstellungsrahmungen. Das Ausmaß der Komplexität wird deutlicher, wenn weitere „Humankategorisierungen“ (Hirschauer 2014, 2017) hinzukommen:

Geschlecht oder Rasse werden als lebenslange Konstanten erwartet, während Alter immanent transitorisch ist und bei Klassen und Nationen an Mobilität zumindest gedacht wird. Leistung zielt auf die Herstellung 
von Individuen, während Geschlecht auf die von Paaren, Ethnizität, Nationalität und Religion auf die von Kollektiven zielen. Daher sind letztere oft mit Segregation verknüpft, während Geschlechter eher durch Kohabitation zusammengefügt werden (Hirschauer 2014: 171).

Letztlich zielt „Humankategorisierung“ (ebd.) darauf ab, die Komplexität der sozialen Lebenswelt für die Erfahrenden zu minimieren, da „Kategorien [...] also grundlegend zu kultureller Ordnung bei[tragen]" (Hirschauer 2014: 172). Im Dickicht der Simplifizierung sozialer Komplexität kommen weitere Aspekte dazu: Verknüpft mit gesellschaftlichen Sphären unterliegt soziale Differenz Schwankungen, sodass soziale Zuschreibungen individuell betrachtet nicht für den gesamten Lebenslauf persistent sein müssen. So setzt eine Aboder Aufwertung eines sozialen Merkmals auch andere Effekte voraus. Zum Beispiel sinkt das Einkommen von Frauen allgemein erst mit Beginn einer Mutterschaft, da quantitativ häufiger junge Mütter in Teilzeit arbeiten und den Anschluss an ihre männlichen Altersgenossen verlieren. Nachteilige Klassenzugehörigkeiten können durch Bildungs- und Statusaufstiege abgemildert werden, haben aber auf der Mikro-Ebene als subjektiv bedeutsame Erfahrungen weiterhin eine hohe Strahlkraft (vgl. Altendorf 1978; Dippelhofer-Stiem 2017); was ähnlich für einen Migrationshintergrund gilt (vgl. Sezer/Dağlar 2009). Aus ethnomethodologischer Forschungstradition stellt sich die grundlegendere Frage, inwiefern scheinbar askriptive Merkmale wie „Geschlecht“ oder „Ethnizität“ in die jeweils gegensätzliche Kategorie konvertierbar sind bzw. künstlich hergestellt werden, wie in Forschungen zu transidenten Personen (vgl. Goffman 1994) oder analog dazu zu trans-ethnischen Zugehörigkeiten (Brubaker 2016) beobachtet werden kann. ${ }^{10}$ Werden diese als veränderbar dargestellt und von Individuen auch verändert („,männlich“ $\rightarrow$,,weiblich“; ethnisch „schwarz“ $\rightarrow$ ethnisch ,weiß“, sowie umgekehrt), zeigt sich in den Erfahrungsberichten der Betroffenen die Erschütterung, die sie durch ihr Verhalten in weiten Teilen des sozialen Raums auslösen.

An dieser Stelle geht es allerdings nicht um die soziologische Relevanz einer jeden Differenzkategorie und auch nicht um die vollumfängliche Herausarbeitung der Herstellungsweise einzelner oder überkreuzter Differenzkategorien in jeder Lebenslage (zum Beispiel bei Brubaker 2015). Diese müsste freilich für jedes Erkenntnisinteresse mit je dem eigenen Bezugsrahmen neu gedacht, abgesteckt und empirisch operationalisiert werden (siehe Wimmer: ,in

10 In der englischsprachigen Literatur wird gerne das Beispiel der afroamerikanischen Aktivistin Rachel Dolezal angeführt, die von ihren Eltern im Sommer 2015 als ,ethnisch weiß“ ,geoutet" wurde. Ähnliche Perspektiven eröffnen sich in anderer Richtung im europäischen Kontext bei der Betrachtung diverser Führungsfiguren rechtsextremer und rassistischer politischer (einheimischer) Gruppen, die ,eigentlich` einen Migrationshintergrund haben. 
a particular domain“, 2008: 1029). Für die Analyse der Herstellungsweise sozialer Differenz werden die „lebensweltliche[n] Kategorien nicht als Ressource soziologischer Kategorisierungen [ge]nutzt, sondern tiefer [...] an[ge]setzt, indem sie das Kategorisieren selbst zum Gegenstand (der empirischen Untersuchung, Anm. NDS) macht" (Hirschauer 2014: 172) Damit soll eine Essentialisierung von Beginn an verhindert werden. Bezugnehmend auf Reckwitz spricht Hirschauer hier von einer ,Kontingenzperspektive auf soziale Phänomene" (ebd.), wobei dieser in seiner Forschung auf ein kulturwissenschaftliches Forschungsprogramm abzielt, das die ,in der Regel nicht bewussten symbolischen Ordnungen, kulturellen Codes und Sinnhorizonte zu explizieren" (Reckwitz 2008: 17) versucht. Insgesamt ist es eher die Kultursoziologie, die ein Repertoire an Ansätzen hervorgebracht hat, mit denen sich Phänomene der Differenzmarkierung erklären lassen. Neben dem Ansatz des „boundary making“ wäre jener der „Hybridität“ zu nennen, die im Laufe des Kapitels genauer vorgestellt werden. Bei dem Aspekt des „Hervorbringens“ als sozialem Prozess drängt sich schließlich der „Un/Doing Difference“-Ansatz auf. Dieser wird vorab auf ihren Gehalt hinsichtlich für die Bearbeitung der vorliegenden Studie überprüft.

Weiter darf die Perspektive der sozialen Ungleichheitsforschung nicht fehlen, geht es im aktuellen Forschungsvorhaben wie oben beschrieben nicht um bloße „Verschiedenartigkeiten“, sondern um soziale Unterscheidungen, die Menschengruppen bevorteilen oder eben benachteiligen (vgl. Solga/Berger/Powell 2009: 16). Beim Diversity-Konzept sind die theoretischen Überlegungen zu sozialer Differenz erstmalig auf einer Meso-Ebene angesiedelt und sollen für die vorliegende Arbeit ebenfalls gesichtet werden. Für eine ungleichheitsrelevante Betrachtung sozialer Differenz ist die Intersektionalitätsforschung hervorzuheben, die eine mehrdimensionale Verschränkung von Differenzkategorien vornimmt.

Die Frage nach der Herstellungsweise sozialer Differenzmarkierung ist eine, die die soziale Konstitution von Gesellschaft, insbesondere von sozialer Ungleichheit, von innen heraus analysiert. Sie geht davon aus, dass die Verteilung ökonomischer und kultureller Ressourcen sowie formaler Machtpositionen für diejenigen vorteilhaft ausfallen, die Deutungshoheit über die Herstellung sozialer Differenzkategorien innehaben. Diese Ausgangsposition beinhaltet zwei Prämissen: Zum einen hat es eine Gruppe von Menschen geschafft, Macht zu besitzen und diese vorteilhaft für die eigenen Zwecke einzusetzen. Von diesem Zusammenhang geht auch Barlösius (vgl. 2004: 65) in Anlehnung an die Studie „Etablierten-Außenseiter-Beziehungen“ von Elias (1969) aus. Des Weiteren handelt es sich durch Menschenhand hergestellte soziale Positionssetzungen von Menschengruppen durch Dritte, die die Deutungshoheit 
über Vorstellungen von „höherwertig(er)“(Arbeit/Qualifikation/Lebensstil/Intelligenz usw.) und ,minderwertig(er)“(Arbeit/Qualifikation/Lebensstil/Intelligenz usw.) innehaben. Die Durchsetzung von Deutungshoheit über die Bewertung bestimmter sozialer Merkmale dienen der Herstellung oder Verfestigung eines bestimmten Hierarchiegefüges. Bei der Modellierung eines Konzepts sozialer Differenz ist die Kritik von Barlösius zu beachten, die besagt, Soziologinnen und Soziologen

tun so, als seien die äußerlichen Merkmale [wie z. B. Geschlecht oder
Ethnizität, Anm. NDS] und die kollektiven Identifikationen selbst Res-
sourcen, über die zu verfügen soziale Vorteile und Nachteile mit sich
bringt. Tatsächlich ist jedoch die gesellschaftliche Bewertung dieser
Kennzeichen entscheidend dafür, ob aus ihnen Nutzen gezogen werden
kann oder mit ihnen Einschränkungen verbunden sind. Deshalb ist zu
untersuchen, welche Gruppen ihre Bewertungen gesellschaftlich durch-
setzen können (2004: 66, Hervorh. durch NDS)

So fällt unweigerlich der Blick auf diese Gruppen bzw. Akteure, die die Durchsetzungsfähigkeit für diese Bewertungen wirkungsvoll vornehmen und für die Gesellschaft an der ,eigene[n] Ordnung der Wahrheit“ (Foucault 1978: 51) mit modellieren. Wer oder was sind solche Akteure? Akteure können neben „Individuen" auch kollektive oder korporative Akteure sein (vgl. Schimank 2013: 30), die sich in Organisationen, Institutionen oder Kleingruppen einfinden, um eigene Interessen oder Emotionen zu artikulieren und durchzusetzen (vgl. ebd.). Über Art und Umfang der „Einmischung“ des Akteurs entscheidet neben seiner Selbsteinschätzung über die eigene Durchsetzungsfähigkeit auch der Umfang der Ressourcen (Geld, Wahrheit), die ihm zum Handeln zur Verfügung stehen (vgl. ebd.). In dieser Funktion ergeben sich für ihn „Gestaltungsfreiräume“. Doch gilt dies nicht allein.

Die schon früher gewachsene Einsicht der Moderne, dass alle gesellschaftlichen Verhältnisse Menschenwerk sind, hat den Schluss auf eine umfangreiche Gestaltungskompetenz - im dreifachen Sinne von Vermögen, Befugnis und Verantwortung - getragen: Wer hervorbringt, kann gestalten; und wer gestalten kann, kann und muss verbessern (Schimank 2013: 18, Hervorh. im Original).

Aus der Gestaltungsfreiheit wird Gestaltungskompetenz: Rechte bringen Pflichten mit sich. Letztlich ,werden die Gesellschaftsstrukturen durch handelndes Zusammenwirken in Akteurskonstellationen beständig hervorgebracht" (Schimank 2013: 31, Hervorh. aufgehoben). 
Auch Stiftungen sind organisationale Akteure, die im Spannungsfeld von Staat und Gesellschaft einen eigenen Platz einnehmen und aus dieser Perspektive gesellschaftliche Verhältnisse modellieren möchten. Es wird daher angenommen, dass die Bedarfe für solche zielgruppenspezifischen Projekte auch aus öffentlichen Diskussionen rund um ,prekäre Lebensverhältnisse“ und mit auf Migration zurückzuführende Probleme begründet werden. Aufzuzeigen ist ferner, welche weiteren ,Achsen der Ungleichheit“" (Klinger/Knapp/Sauer 2007) für eine Konzeption sozialer Differenzmarkierung relevant sein können als die in der Literatur mit Geschlecht, Klassenlage und Migration bzw. Ethnizität prominent vertretenen.

Wie ist nun weiter vorzugehen? Bisher wurden die Fallstricke für die Modellierung eines Analyserahmens zu sozialer Differenz erörtert. Weiter fokussiert auf die Fragestellung, wie soziale Differenz in der operativen Arbeit von Stiftungen hervorgebracht wird, ist nach einer Herangehensweise zu suchen, die in den Blick nimmt, was Differenzmarkierungen in ihrer Hervorbringung kennzeichnet. Dem eingangs behandelten konstruktivistischen Paradigma folgend fällt die Wahl zunächst auf Ansätze, die die grundsätzliche Konstruiertheit gesellschaftlicher Verhältnisse in den Blick nehmen (3.2). Dazu gehört neben den sozialkonstruktivistischen Grundlagen (3.2.1) der „Un/doing difference"-Ansatz, der ebenfalls diesem Forschungszweig zugeordnet wird (3.2.2). Hiernach folgen Konzepte, die konkret die Differenzbenennung und Vermengung fokussieren und dabei bedeutende Ungleichheitsmerkmale in ihrer Gleichzeitigkeit behandeln (3.3): Zum einen werden die kulturwissenschaftlichen Grenzziehungskonzepte, das „boundary making“ und das „Othering" vorgestellt (3.3.1). Darauf folgen Ausführungen, die hybride Identitäten aufgrund der Nicht-Übereinstimmung von ethnischer und nationaler Zugehörigkeit thematisieren und zumeist als Kritik an bestehenden Forschungsparadigmen zu verstehen sind (3.3.2). Im Anschluss an den zur Ungleichheitsbetrachtung zugeordneten Topos der Intersektionalität (3.3.3) wird abschließend der eher anwendungsorientierte Diversity-Ansatz (3.3.4) erörtert. Beide Ansätze tun sich explizit damit hervor, eigenständige Differenzmerkmale zu einem kohärenten Modell zu verknüpfen. Um den theoretischen Rahmen insgesamt abzustecken, gilt es, diese diversen Ansätze zu einem Analyserahmen zu verdichten (3.4), die, wie in diesem Abschnitt oben beschrieben, aufgrund ihrer eigenen Forschungstraditionen selten gemeinsam gedacht werden (vgl. Hirschauer 2014, 2017; Riegel 2016). So soll die Bearbeitung der Fragestellung zur Herstellungsweise sozialer Differenz ermöglicht werden. 


\subsection{Sozialkonstruktivistische Ansätze sozialer Differenz}

\subsubsection{Sozialkonstruktivistische Grundlagen}

Die Frage danach, wie sich eine Gesellschaft konstituiert, wie sie „Wahrheiten" über soziale Gegebenheiten produziert und wie diese verstetigt werden, gehört zu den zeitlosen Fragen der Soziologie. In älteren Schriften der 1960er bis 1980er Jahre ${ }^{11}$ werden in konstruktivistischen Theorieansätzen soziale Gegebenheiten noch als Forschungsperspektive behandelt, in der verhandelt wird, $o b$ soziale Differenz eine Konstruktion ist. An einer Skizze der sehr unterschiedlichen sozialkonstruktivistischen Abhandlungen versucht sich Collin. ${ }^{12}$ Ausgegangen werden muss von der Prämisse,

dass die [...] soziale Wirklichkeit als solche von den Begriffen, mit denen wir sie beschreiben, oder von den Kategorien, in denen wir sie denken, erschaffen worden sein sollte (Collin 2008: 11).

Menschliches Wissen bringt in dieser Lesart soziale Welt hervor, bringt Ordnung in diese und stellt Kategorien bereit, die Individuen für ihre alltäglichen und nicht-alltäglichen Handlungen zur Hilfe nehmen. Collin führt weiter aus, dass der erkenntnistheoretische Konstruktivismus der Auffassung sei,

dass unser Wirklichkeitsbild stets in Begriffen und Kategorien formuliert sein wird, die von anderen Faktoren als dem Gegenstand der Erkenntnis selbst bestimmt sind, meist von den gesellschaftlichen Umständen, denen der Erkenntnisprozess unterliegt (Collin 2008: 26).

Die soziale Kategorie, die durch Performanz hervorgebracht wird, speist sich demnach nicht aus der Beschaffenheit der Kategorie selbst, sondern aus der Konnotation, die dieser Kategorie im Vorfeld des Sprechakts zugerechnet worden ist und das einen gewissen Grad an Allgemeingültigkeit erreicht hat. Es entspricht dem soziologischen „Commonsense, soziale Wirklichkeit sei von Menschen gemacht“ (Hirschauer 2011: 102). Es drängt sich aber die Frage auf, durch welche genauen Umstände diese Reproduktionen erfolgen. Knorr-Cetina führt vor, welche „Spielarten des Konstruktivismus“ (1989) vorhanden sind, bei der es nicht mehr um das ,Was', sondern um das, Wie' sozialer Ordnungen geht. Sie stellt die Leitfrage:

11 Berger/Luckmann 1997 (1966); Lofland 1979 u. a.

12 Eine solche Übersicht liefert Finn Collin in seinem Werk „Konstruktivismus für Einsteiger“ (2008, Paderborn), Übersetzung aus dem Dänischen 
WIE wird eine Kategorisierung, eine Unterscheidung oder Segmentierung der Welt von den Teilnehmern operationalisiert? (Knorr-Cetina 1989: 92).

Auch Soeffner plädiert für „Rekonstruktion statt Konstruktivismus“ (1992), indem er kritisiert, älteren Ansätzen, wie jenen von Berger/Luckmann gelinge es nicht, „konkret-historische, gesellschaftliche Konstruktionen [...] von Wirklichkeiten in den Blick zu bekommen" (Soeffner 1992: 479).

Erstmalig angewendet auf ein konkretes Differenzmerkmal finden sich sozialkonstruktivistische Ansätze mit dem Fokus auf „Geschlecht“. Neben der makro-soziologischen, stärker feministischen Perspektive zur unterschiedlichen Platzierung und Positionierung von Frauen und Männern, Jungen und Mädchen im Bildungssystem, Arbeitsmarkt, Gesundheitssystem oder in der Privatheit, meist zu Ungunsten von Frauen und Mädchen, fallen in der MikroPerspektive insbesondere die sozialkonstruktivistischen und ethnomethodologischen Ansätze rund um ,doing gender“-Konzepte (West/Zimmerman 1987) auf. Dabei wird das soziale Geschlecht, gender, als eine soziale Differenzierungskategorie verstanden, die omnipräsent den sozialen Raum strukturiert (u. a. Goffman 1994). ,In der Beherrschung der entsprechenden Praktiken erweist sich die (geschlechtsbezogene) Handlungskompetenz der Gesellschaftsmitglieder" (Meuser 2010: 151). Angelehnt an die Arbeiten von Garfinkel (1967) zu einer transsexuellen Person sowie an ethnologische Studien von Mead (1958) zu sehr unterschiedlichen Konnotationen von Weiblichkeit und Männlichkeit in globaler Perspektive zeigt sich, dass die Hervorbringung von Geschlecht erstens in eben diesen Alltagspraktiken von den Individuen selbst bewusst, unbewusst und ständig durch Gestik, Mimik, Körperhaltung und Gesprächsthemen reproduziert wird. Zweitens werden durch Zuschreibungen bzw. Etikettierungen Charakterzüge, Verhaltensweisen, Stärken und Schwächen sowie Interessen zu je „weiblichen“ bzw. „männlichen“ gemacht. Dabei sind seitens der Untersuchungssubjekte insbesondere Argumente in Rekurs auf die „Natur" die Regel, machen sie doch die verwendete Zuschreibung scheinbar unumkehrbar und unabwendbar. Die als „Institution“ bezeichneten Deutungen kollektiven Ausmaßes fungieren als „Regulativmuster menschlichen Handelns und menschlichen Zusammenlebens [...], und zwar insbesondere solche, die durch eine Verankerung in den Handlungsorientierungen und Sinngebungsmustern der Gesellschaftsmitglieder gekennzeichnet sind" (Gildemeister/Wetterer 1992: 237, Hervorhebung im Original). Da sozialen Ausformungen, die kulturell begründet werden, einer Retrospektive zugänglich sind (vgl. ebd.: 228), kann dabei der „Konstruktionsmodus eher [durchschaut]“ (ebd.) werden. Bezogen auf die „Sinngebungsmuster“ (ebd.: 237) seitens der Gesellschaftsmitglieder ergänzen Gildemeister/Wetterer: 
Angesprochen ist damit die grundlegende Ebene der interaktiven Herstellung sozialer Wirklichkeit; Interaktion in diesem Sinne ist kein Medium, in dem mehr oder weniger vorsozial gedachte Personen [...] mitoder auch gegeneinander handeln, sondern stellt einen (formenden) Prozeß eigener Art dar, eine eigene Wirklichkeit der handlungspraktischen Realisierung generativer Muster und Regeln (Gildemeister/Wetterer 1992: 230, Hervorhebungen im Original)

Festzuhalten ist: Die soziale Wirklichkeit ist menschgemacht. Singulär getätigte Narrationen und Interaktionen in sozialen Gegebenheiten führen dazu, dass sie einen Raum schaffen für die Generierbarkeit einer gegenseitigen Wirklichkeit mit eigenen Regeln und Strukturen. Diese Grundsätzlichkeit und Übertragbarkeit der Wirklichkeitskonstruktionen bezogen auf ein Differenzmerkmal fand sich, wie beschrieben, erstmalig in der Geschlechterforschung. Eine plausible Annahme geht dahin, dass dies auch für andere Differenzmerkmale gültig ist.

\subsubsection{Un/doing difference-Ansätze}

Die Grundlagen der sozialkontruktivistischen Ansätze weisen auf die „Reproduktionsbedürftigkeit" sozialer Grenzziehungen hin und machen deutlich, dass diese auf verschiedenen Ebenen sozial ,gemacht" sind und in nahezu allen gesellschaftlichen Sphären nachhaltig Wirkung zeigen. Was bedeutet, gemacht sein' und wie könnte die Funktionsweise eines ,Machens' aussehen?

Einen Vorschlag für eine konkrete Handhabe sozialkonstruktivistischer Ansätze liefert das ethnomethodologische Doing-difference-Konzept von West/Fenstermaker (1995), bei der zum Ziel gesetzt wurde, Differenzkategorien in ihrer Interaktion konzeptionell gemeinsam zu denken. Die Autorinnen arbeiteten heraus, inwiefern race, class und gender eingebettet in Alltagspraxen hergestellt werden (können) und wie sie miteinander verwoben sein (können). In Bezug zur Kategorie Geschlecht führen sie an, dass Mitglieder einer Gesellschaft Zuschreibungen routiniert fabrizieren und sie diese letztlich mit einer Rahmung versehen (,,place them into a social framework", West/Fenstermaker 1995: 21). Die Hervorbringung von „Ethnizität“ folgt dieser Argumentation gemäß nach einem ähnlichen Muster: Diese wird als ,,praktische Hervorbringung“" (accomplishment) (West/Fenstermaker 1995: 23) der ethnisch divergenten Personen bezeichnet, bei dem die Divergenz durch die Interaktion mit dem sozialen Umfeld entsteht, also einen Widerhall in der sozialen Umgebung erhält. Die Zugehörigkeit zur Klassenlage gestaltet sich dagegen etwas komplizierter: Während sich Geschlecht (gender) und Ethnizität (race) plausibel 
als Folge von Interaktionen kennzeichnen lassen, erscheint Klasse als eine Kategorie, die scheinbar Leistung und Geschick von Individuen objektiv misst (vgl. West/Fenstermaker 1995: $27 \mathrm{f}$., ,if someone is poor, we assume it is because of something they did or did not do", ebd., Herv. i. O.). Gleichwohl weisen die Autorinnen darauf hin, dass staatliche Stellen gerade in öffentlichen Programmen der sozialen Fürsorge weit mehr erfassten als den simplen Fakt ökonomisch bedingter Armut. Bei der Vergabe von öffentlichen Geldern an Hilfebedürftige entscheiden sie immer in Zusammenhang mit gesundheitlichen Einschränkungen, Elternschaft und schwerwiegenden Lebensereignissen (vgl. ebd.). Somit wäre anhand dieses Beispiels der klassenspezifische Platzierungsvorgang als ein Konglomerat aus biographischer Prägung, gesundheitlicher Verfassung, Lebensentscheidungen und Befähigungen zu verstehen. Das Fazit lautet, dass die Klassenlage in Bezug zu anderen Faktoren gesetzt werden muss, sie entsteht nicht aus sich selbst heraus. Für die Diskussion um die alltägliche Hervorbringung von Differenz schlussfolgern West und Fenstermaker:

(C)onceiving of these as ongoing accomplishments means that we cannot determine their relevance to social action apart from the context in which they are accomplished (West/Fenstermaker 1995: 30).

Die Herstellung der Differenz erfolgt im Doing-difference-Ansatz in erster Linie durch die Protagonisten selbst, sie wird nicht (nur) durch Dritte hervorgebracht. Daher sind ,ihre weiteren Annahmen [...] sozialtheoretisch eng auf Interaktion beschränkt und institutionell, verlängerungsbedürftig“" (Hirschauer 2014: 182). Sie bieten eine Idee davon, wie die Herstellung sozialer Differenz mikro-perspektivisch aussehen kann.

Hirschauer ergänzt hierzu in seinem Un/doing-difference-Ansatz die Idee, dass Differenzdimensionen je nach Analyserahmen zu „entrümpeln“ sind, da soziale Merkmale in bestimmten Situationen weniger relevant sein können bzw. „ruhen“ (Hirschauer 2014: 183) können. Soziale Differenz sei „ein kontextabhängiges Ereignis, das phasenweise intensiv sein kann" (ebd.). So kann bei der Betrachtung der Kategorie „Geschlecht" diese für einen bestimmten Bereich eine überaus wichtige Rolle spielen, während die des Alters in den Hintergrund tritt. Umgekehrt können sich in bestimmten Analyserahmungen die Kategorien „Ethnizität“ und „Klasse“ vehement aufdrängen, in deren Kontext die Kategorie „Geschlecht" eine vernachlässigbare soziale Dimension bildet. „Am Rande des undoing findet der Wechsel zu anderen Unterscheidungen statt" (Hirschauer 2014: 183), die dann wiederum als lose Enden fungieren und in neue Feldabmessungen für weiterführende Forschungsprozesse überführt werden müssen. Wie tiefgreifend sprachliche Implikationen soziale Differenz- 
merkmale überkreuzen lassen können, illustriert Hirschauer (2014: 184) anhand eines Beispiels: Während sich im Ausdruck „Dame“ die Kategorien Geschlecht und Klasse kreuzten, stelle der Begriff „Mädchen“ Geschlecht und Alter in den Vordergrund. Wird der Ausdruck ,mädchenhaftes Verhalten“"verwendet, könne von „,doing gender while doing age“ (ebd.) gesprochen werden. Es wird eine Bewertung eines geschlechts- und altersspezifisch nicht angemessenen Verhaltens einer beliebigen Person vorgenommen. So werden soziale Kategorien in Ausdrücken verwoben, hergestellt, abgeschwächt und etikettiert.

Die unter 3.2 vorgestellten Ansätze sind insoweit gewinnbringend, als sie die menschliche Hervorbringung von sozialen Dichotomien in verbalen und nonverbalen Aktionen deutlich machen. Diese erfolgen mit der Zeit nach bestimmten Mustern der Sinngebung und kerben sich sodann in die sozialen Strukturen des allgemeinen Miteinanders ein. Sie weisen außerdem auf die Fragilität der expliziten Dichotomien hin, die im hohen Maße kontextabhängig hinsichtlich Raum, Zeit und Umstände sind. Letztlich zeigen sie die Komplexität gemachter sozialer Differenz auf, da sie allesamt andeuten, dass es, anders als in klassischen sozialwissenschaftlichen Theorieansätzen, immer mehr als eine Differenzkategorie geben muss, die für das genaue Verständnis der sozialen Hervorbringung von Differenz relevant ist. Diese müssen nicht explizit genannt worden sein, sondern verstecken sich in der Semantik der verwendeten Begriffe („Dame“). Schlussendlich deuten sie an, wie vehement soziale Verhältnisse durch die Hervorbringung sozialer Differenz verstetigt wird.

\subsection{Differenzbenennung \& Vermengung}

\subsubsection{Kulturwissenschaftliche Grenzziehungskonzepte}

Neben den Ansätzen des Un/doing difference sollen zwei kulturwissenschaftliche Konzepte begutachtet werden, da sie besonders die Benennung sozialer Differenz in den Fokus rücken. Sie sollen als "Grenzziehungskonzepte“ vorgestellt werden, da bei ihnen die Illustration der Grenzmarkierung eine zentrale Rolle spielt.

Boundary making

Zunächst soll das unter anderem auf Wimmer (2008) und Lamont/Molnár (2002) zurückzuführende „boundary making“ auf seinen Gehalt für die vorliegende Studie hin untersucht werden. Grundlegend analysiert Wimmer (2008) 
die Entstehungslogik und Funktionsweise der Grenzziehungsmetapher auf ethnische Gruppen, Migration und Nationen sowohl im globalen wie auch im Kontext individueller Interaktionen. Zum Ende seiner Ausführungen deutet er an, dass dieser Versuch noch nicht Auswirkungen von Grenzziehungen klären kann (vgl. Wimmer 2008: 1046). Mit mehr als der ethnischen Differenzkategorie befassen sich Lamont/Molnár (2002). Sie arbeiten eine vielschichtige Herangehensweise unter Berücksichtigung der Unterschiede zwischen dem US-amerikanischen und dem europäischen Kontext (vgl. Lamont/Molnár 2002: 186) heraus. Zunächst ist die Unterscheidung hervorzuheben, die sie zwischen ,symbolic boundaries“" und ,social boundaries“" explizieren. So seien erstere dazu gedacht, dass durch soziale Akteure (vgl. Lamont/Molnár 2002: 168) „objects, people, practices, and even time and space“ (ebd.) kategorisiert und mit deren Hilfe eine Übereinkunft über Realität erzielt werde (vgl. ebd.). Weiter heißt es:

Symbolic boundaries also separate people into groups and generate feelings of similarity and group membership. [...] They are an essential medium through which people acquire status and monopolize resources (Lamont/Molnár 2002: 168).

Die symbolische Grenze verweist also auf Differenzmarkierungen, die durch normative Zuschreibungspraxen erfolgen. Dadurch manifestieren sie Zugehörigkeiten und die Wahrnehmung von Gemeinsamkeiten innerhalb dieser Gruppen. Mit einer quantifizierbaren Ungleichheit haben diese wenig gemein. Dagegen bilden ,symbolic boundaries' eher die objektivierbaren sozialen Ungleichheiten und ungleiche Privilegien (vgl. ebd.). Bei der Analyse der Handhabe sozialer Differenz wäre daher das Bild der symbolischen Grenze zu wählen, da es hier um die symbolische Herstellungspraxis sozialer Unterschiede geht. Allerdings sind beide in gewisser Weise verknüpft, da symbolische Grenzen, salient behandelt, zu sozialen Grenzen werden können (vgl. Lamont/Molnár 2002: 186). Als Beispiel wird Deutschland mit seiner Ost-/WestVergangenheit genannt. So fruchtbar die Ansätze sind, so resümieren Lamont/Molnár (2002: 187) auch hier, dass es eine Forschungsaufgabe sein sollte, genauer ins Auge zu fassen, wie soziale Akteure soziale Gruppen als ähnlich oder unterschiedlich hervorbringen und wie sie ihre Verantwortung diesen gegenüber begründen. Für die Meso-Ebene der institutionellen Repräsentation zivilgesellschaftlicher Organisationen ist schließlich der Hinweis hilfreich, dass in Anlehnung an Abbott (1988): ,professions constitute an open, ecological system in which individual professions exist in independence" (Lamont/Molnár 2002: 178), sodass davon auszugehen ist, dass Berufsgruppen oder organisationale Einheiten ein eigenes Repertoire an Sprachcodes kreieren, 
um soziale Phänomene in Übereinkunft zu beschreiben. Letztlich wird für statusdifferente Gruppen konstatiert: „We need to focus especially on hidden assumptions concerning the measuring sticks“"(Lamont/Molnár 2002: 188).

\section{Othering}

Im kulturwissenschaftlichen Programm sind für das vorliegende Forschungsinteresse in erster Linie Ansätze der sogenannten Postcolonial Studies zu erwähnen, die aus einer rassismuskritischen Perspektive heraus bestehende gesellschaftliche Schieflagen in den Blick nehmen (vgl. Hall 1999, Hall 2004, Bhabha 1994) und diese weitestgehend als eine Art „DifferenzbenennungsAnsatz" vorstellen. Während in den interaktionistischen Annahmen der Fokus noch sehr auf die Sozialkategorie „Geschlecht“ gesetzt wird, da diese Theorien stärker von Geschlechterforschenden entwickelt werden, spielt beim Konzept des „Otherings“ in erster Linie die Bedeutungsaufladung ethnischer Zugehörigkeiten eine Rolle und versteht das Forschungsprogramm als Kritik an bestehenden „rest-kolonialen“ Zuständen. „Mit dem Begriff des Othering wird die Konstruktion der_des Anderen als Prozess des ,Different-Machens' (Castro Varela/Dhawan 2005: 60) markiert, der sowohl Elemente der Festschreibung, der Ausgrenzung als auch der Unterwerfung enthält" (Riegel 2016: 52). Dabei wird auf verschiedenen Ebenen und durch verschiedene Akteure „eine bestimmte Gruppe erst als solche, dann als Andere diskursiv hervorgebracht und identitär festgeschrieben" (ebd.). In der Tradition der Postcolonial Studies erfasst das Konzept die soziale Dichotomie eines „Eigenen“ (wir) und eines „Fremden“ (die anderen). Im deutschsprachigen Kontext führt Paul Mecheril die Bezeichnungspraxis von „Menschen mit Migrationshintergrund“ oder schlicht „Migranten“ als Markierung von „Migrationsanderen“ (1999) ins Feld. Er plädiert dafür, alternativ zur klassischen Migrationsforschung (Integrationsforschung) eine Perspektive einzuführen, die sich ,kritische Migrationsforschung" (2013) nennt, in der zumindest im erziehungswissenschaftlichen Kontext die „Subjektbildung“ (2014) von Minoritäten gerade in Forschungskontexten berücksichtigt bzw. vermieden wird.

Die Konzepte sind als Kritik an Hegemonien auf Grundlage ethnischer Zuschreibungen formuliert und bieten einen Analyserahmen für Bezeichnungspraxen an. Es zeigt eine differenzierte Sichtweise der Herstellung von Kollektivismen auf und formuliert sie als Kritik. Dieser Punkt wird im Folgenden vertieft. 


\subsubsection{Forschungsansätze als Kritik am Bestehenden}

Hybridität

Inspiriert von den Postcolonial Studies ist die deutschsprachige kritische Migrationsforschung dabei, die Vergabe von Minoritätenstatus mehrdimensional zu betrachten bzw. aufzuzeigen, welcher Art blinder Flecken die Migrationsforschung bisweilen produziert (vgl. Mecheril 2014, Mecheril u. a. 2013b; Reinprecht/Latcheva 2016; Hetfleisch 2013). Çetin spricht sogar vom Vorhandensein einer „weißen Sozialwissenschaft“ (Çetin 2013; ähnlich Kaloianov 2013), Canan vom methodologischen „Nationalismus in der Migrationsforschung" (Canan 2015: 39 in Anlehnung an: Wimmer/GlickSchiller 2002). Für den deutschsprachigen Migrationsdiskurs wird aus den Ausführungen geschlossen, dass im

Gegensatz zu den gängigen nationalen Narrativen [...] im postmigrantischen Diskurs nicht nach integrativen Leistungen von (Post-) Migranten gefragt [wird], es rücken vielmehr Prozesse von Entortung und Neuverortung, Mehrdeutigkeit und Grenzbiographien ins Blickfeld (Yıldız 2014: 21).

In dieser Lesart müsse „Migration als Perspektive, nicht als Gegenstand begriffen“ (Y1ldız 2014: 22) werden. Die theoretische Modellierung ist im vollen Gange. Dieser Paradigmenwechsel, der in den Kulturwissenschaften angeregt wird und durch die kritische Migrationsforschung einen Niederschlag in den Erziehungswissenschaften erhält, wird in den Sozialwissenschaften seltener verhandelt, sodass sich Konzeptionen rund um die Repräsentationen und Herstellungsweise sozialer Differenz mit Fokus auf migrantische Minoritäten noch auf die Hilfe von Nachbardisziplinen angewiesen sind.

Beziehen sich die zu Beginn thematisierten sozialkonstruktivistischen Ansätze auf die Kategorie „Geschlecht“, stellen die vorgestellten Konzepte rund um Hybridität und Postmigration das Differenzmerkmal „Ethnizität“ in den Vordergrund. Einschlägige „Nachbarkategorien“, etwa die Religion, die mehr oder weniger im Zusammenhang mit „Ethnizität“ vermutet werden, werden tangiert.

An dieser Stelle stellt sich die Frage, wie genau dann ,Ethnizität“ im Kontext sozialer Differenzanalyse zu verstehen ist. ${ }^{13}$ In Anlehnung an Max Weber definiert Mathias Bös Ethnizität im Kern als „,soziale Handlungen [seitens einer Bevölkerungsgruppe, Anm. NDS] [,die] auf einer realen oder mythischen

13 Abgrenzungen zu Begriffen wie „Nation“, „Herrschaft“, „Minderheit“ sowie historisch-politische Herleitungen weiterführend bei Stender 2000 sowie Sökefeld 2007. 
gemeinsamen Herkunft [...] aufbauen“ (Bös 2008: 57). Damit gelingt auch, so Bös, die Unterscheidung gegenüber einem Fanclub oder der Frauenbewegung (vgl. ebd.), die letztlich auch gemeinsame Interessen, Rituale oder politische Standpunkte vertreten. Weiter heißt es:

Nicht Ethnizität ist der Grund für eine gemeinsame Lebensweise, ein Gruppenschicksal oder gemeinsames Aussehen, sondern weil diese Merkmale vorliegen, entsteht „Ethnizität“, im Sinne des geteilten Abstammungsglaubens, der dann zur „Propagierung“ eines spezifischen ethnischen Gruppenhandelns verwendet wird (Bös 2008: 57).

Somit wird Ethnizität als soziale Praktik verstanden. Sie muss, wie Gümen bereits Ende der 1990er Jahre formuliert (siehe Abschnitt „Intersektionalität", 3.3.3), in ihrer Herstellungsweise betrachtet werden, damit es keine Essentialisierung erfährt und muss ,notwendigerweise immer in einer aktuellen gelebten Situation“"(Bös 2008: 61) fokussiert werden. Daher erscheint es nur schlüssig, dass laut Bös ,,(t)echnisch gesprochen [...] Ethnizität eine abhängige und keine unabhängige Variable“ (Bös 2008: 57) ist. Ähnlich formuliert es Sökefeld, wenn er davon spricht, dass Ethnizität nicht die Ursache für Differenz ist, sondern soziale Handlungen Ethnizität performieren (vgl. Sökefeld 2007: 31). Soziale Gruppen, die als ethnisch different gelten, können einen Migrationsstatus haben. Sie müssen es aber nicht zwangsläufig. Dies gilt allerdings besonders dann, wenn Ethnizität an eine religiöse Praxis oder mit einer Migrationsnarration verbunden wird, was zumeist über mehrere Generationen hinweg Bestand haben kann, auch, wenn der Migrationsstatus sozio-strukturell und rechtlich längst obsolet geworden ist. Daher

rekurriert das Postmigrantische auf das Nachwirken von Migration über die Generationen hinweg -sei es als personales, familiäres oder gesellschaftliches Narrativ, als Ordnungskriterium sozialer und gesellschaftlicher Macht, als Zugangsbarriere zur Definition nationaler Identität oder als Konfliktlinie ideologischer Positionierung (Foroutan 2016: 231).

So ist zu erklären, warum die breite Migrationsforschung beständig um den Aspekt der Religionszugehörigkeit kreist ${ }^{14}$ neben dem Aspekt, dass öffentliche Diskussionen rund um ethnische und religiöse Minderheiten einen gegenüber anderen Themen und Disziplinen ungewöhnlich starken Widerhall in der entsprechenden gesellschaftswissenschaftlichen Fachliteratur erhält. „Postmigrantisch wird als Begriff derzeit vor allem im deutschsprachigen Raum als

14 U. a. Kazzazi u. a. 2016; Tiefensee/Kraft 2011; Graf 2006; Müller 2013; Projektgruppe heymat der Humboldt Universität Berlin. 
neue, explorative akteurs- und gesellschaftsanalytische Perspektive verwen-

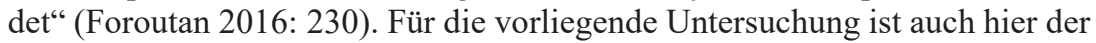
akteurstheoretische Ansatz rund um die Aushandlungsprozesse postmigrantischer Akteure in biographischer Perspektive zweitrangig. Die Makro-Ebene reißt allerdings ein Feld auf, das dadurch gekennzeichnet ist,

die Trennlinie des Migrantischen als bedeutungslos zu betrachten und Gesellschaftsanalyse neu auf Fragen der ökonomischen, geschlechtsspezifischen, machtorientierten, kulturell und ethnisch bzw. rassistisch legitimierten Ungleichheit zu lenken (Foroutan 2016: 232).

Als Quintessenz steht im Vordergrund, die Benennungspraxis ausgehend von ethnischer Differenz weitläufiger zu betrachten als die engmaschigen klassischen Konzepte von „Ethnizität" und „Migration“, die auf Fragestellungen rund um (kulturelle) Integration verengt sind. Ist die Trennlinie zwischen den „Migranten“ und allen anderen einmal überwunden, eröffnet sich in Anspielung an Bhabha ein neuer Raum, der offen ist für die vielen Ebenen der Machtund Herrschaftsausübung, für die Durchkreuzung sozialer Ungleichheitsachsen sowie die Schärfung der Analyse auf verschiedenen Abstraktionsebenen, von Makro-, Meso- über die Mikro-Sicht gesellschaftlicher Dynamiken. Die empirischen Daten werden zeigen, inwieweit das möglich ist, da sich Stiftungsprojekte ohnehin keiner Sphären- oder Gruppenlogik zuordnen lassen und gesellschaftlich und ebenentheoretisch, bildlich gesprochen, querbeet agieren. Welche neuen Achsen bestimmen dann postmigrantische Forschende, um das Konzept empirisch greifbar zu machen?

$\mathrm{Zu}$ diesem Zeitpunkt kann das Konzept der Postmigration diese hohen Erwartungen an empirische Anwendbarkeit (noch) nicht erfüllen. Allerdings können bereits folgende postmigrantische Fragen behandelt werden: Kommt es also vor, dass in Darstellungen von Stiftungsprojekten auf diese ,Trennlinie“ migrantisch/einheimisch verzichtet oder ethnische Differenz präzisiert wird? Inwiefern kommen Kollektivismen zum Tragen, wie es in den OtheringKonzepten thematisiert wird?

Sollen nämlich soziale Differenzmerkmale auf ihre Präzision mit Blick auf andere Kategorien wie zum Beispiel Klassenlage untersucht werden, ist nach Verschränkungstheorien Ausschau zu halten. Daher folgen zwei weitere Betrachtungen sozialer Differenz-Modellierungen: Auf den derzeit hoch frequentierten Ansatz der „Intersektionalität“, das sich zum Ziel gesetzt hat, einschlägige Differenzmerkmale in ein kohärentes Theorie-Konzept zu fassen, folgt das eher pragmatische Paradigma der „Diversity“. Auch ihre Erträge sollen für die vorliegende Arbeit untersucht werden. 


\subsubsection{Ungleichheitstheoretischer Ansatz Intersektionalität}

Im Rahmen der ungleichheitstheoretischen Ansätze nimmt die Intersektionalitätsforschung eine prominente Rolle ein. Dabei bestehen mittlerweile verschiedene Denkrichtungen, die in erster Linie die Ursprungsidee vom aktuellen Forschungsprogramm trennen.

In ihrem Aufsatz „Demarginalizing the Intersection of Race and Sex: A Black Feminist Critique of Antidiscrimination Doctrine, Feminist Theory and Antiracist Politics“ (1988) eröffnete die afroamerikanische Juristin Kimberlé W. Crenshaw eine neue Perspektive auf feministische Theorien, die zum Ziel hatte, mehr als eine soziale Differenzdimension für die Benennung von Exklusion aufzuzeigen.

I argue that Black women are sometimes excluded from feminist theory and antiracist policy discourse because both are predicated on a discrete set of experiences that often does not accurately reflect the interaction of race and gender (Crenshaw 1988: 140).

Noch bildete die Benennung der Verschränkung „,kein abstraktes Konzept theoretischer Komplexitätsforschung, um die Bedeutung multipler sozialer Ungleichheiten klären zu können, sondern die Beschreibung eines alltäglichen Phänomens" (Schnicke 2014: 4). Allerdings häuften sich in diesem Rahmen disziplinenübergreifend kritische Auseinandersetzungen mit der bis dahin eher „weißen“ feministischen Ansicht zu Gesellschaftskritik bzw. zur Eindimensionalität bei der Analyse ungleicher Gesellschaftsverhältnisse. Crenshaw bringt in diesem Zusammenhang den Begriff der ,intersection“ in die Debatte ein (Crenshaw 1988), der auf der Idee beruht, dass unterschiedliche Differenzmerkmale auf Individuen gleichzeitig (,simultaniously“) wirken. Dabei verwendete sie die Metapher der Verkehrskreuzung, an der soziale Merkmale und Lebenslagen gleichzeitig aufeinandertreffen und sich bedingen (vgl. auch Çetin 2012: 86). ,Schwarze' Wissenschaftlerinnen lehnten additive DifferenzModelle ab, da diese nicht die Funktionsweise komplexer Ungleichheitsverhältnisse ausdifferenzierten, sondern lediglich mit dem Nebeneinander sozialer Kategorien arbeiteten (vgl. Bednarz-Braun 2004: 32).

Für den deutschsprachigen Raum arbeitete Sedef Gümen Ansätze des USamerikanischen Black Feminism auf. Würden nämlich „kulturelle Differenz“ oder unterschiedliche Lebensumstände zwischen Frauen bloß erwähnt, löse dies „nicht das Problem der Essentialisierung dieser Kategorien“ (Gümen 2001: 142). 
Wenn sie das Vorhandensein und Nebeneinanderexistieren dieser Kategorien als ,,askriptive Merkmale“, die frau/man eben hat oder ist, voraussetzt, kann sie den Strukturnormen (z. B. staatliche Regulierungen) von Differenz und den Herstellungs- und Konstituierungsprozessen der „Zugehörigkeit“ nicht gerecht werden. Genauer: Es reicht nicht aus, lediglich auf „ethnische Differenzen“"zu verweisen. Die Gefahr, alten Begrenzheiten verhaftet zu bleiben, bleibt solange bestehen, wie nicht die kontextspezifische gesellschaftspolitische Konstituierung von Differenzen und Ungleichheiten und ihre komplexen Überschneidungen mitgedacht werden (Gümen 2001: 142, Hervorhebungen im Original)

Gümen lenkt hier den Blick auf „Herstellungs- und Konstituierungsprozesse [...] der ,Zugehörigkeit““(Gümen 2001: 142), die für ein Konzept „Geschlecht plus Ethnizität“ entscheidend sind. Daher greife es auch zu kurz ,dualistische Konzeptionen" (ebd.) in den Blick zu nehmen, von der sich schließlich die Geschlechterforschung längst verabschiedet hat. Stattdessen gelte es ,,andere soziale Kategorien in ihrer Interaktion mit Geschlecht zu berücksichtigen“ (Gümen 2001: 143, Hervorhebung im Original). Bereits hier wurde der Versuch unternommen, die Spaltung zwischen Ungleichheits- und Differenzforschung (siehe 3.1) zusammenzuführen.

Den Anfängen der Intersektionalität folgte der Versuch, das Theoriegerüst auf eine gesamtgesellschaftliche Ebene zu ,heben'. Dabei sind bestimmte Lücken zu schließen. So erwähnt Klinger (2008: 38 ff.) drei Achsen der Unterdrückung (,systems of oppression“):

Denn in der Zeit, als sich die drei großen ,systems of oppression “ herausgebildet und den Höhepunkt ihrer Entwicklung erreicht haben, hat eine umfassende Analyse ihrer Zusammenhänge nicht stattgefunden. Die Frage nach dem , macro level connections ' zwischen den drei ,systems of oppression', also die Frage nach den Verhältnissen, in denen sie einander stehen, nach den Arten und Weisen, wie sie mit einander [sic] verbunden sind, wie sie ineinander greifen (,interlocking'), ist damals nicht ernsthaft gestellt und in der Folge bis heute kaum zureichend beantwortet worden (Klinger 2008: 55, Hervorhebungen im Original).

Intersektionalität fungiert bis heute als ein „Label einer Forschungsrichtung, das auf multiple Subjektpositionierungen im Sinne von Diskriminierungs- und Marginalisierungsmechanismen, aber auch im Sinne von Normalisierungsund Privilegierungsmechanismen fokussiert" (Schnicke 2014: 5). Eine empirische Herangehensweise wird zumeist in neueren Forschungsprojekten angestrengt, die mit dem Intersektionalitätskonzept explizit arbeiten. Der Fokus liegt dabei auf der Rekonstruktion von Alltagspraxen oder Biographien, die beispielsweise aus narrativen Interviews gewonnen wurden (zum Beispiel 
Degele/Winker 2009; Çetin 2012; Langsdorff 2012; Paulus 2012; Schrader 2013; Amirpur 2016). Explizit versucht die Intersektionalitätsforschung, „strukturell begründete, in holistischen Ansätzen thematisierte Unterschiede und Diskriminierungen [...] mit individuellen, handlungstheoretischen Ansätzen“ (Salzbrunn 2012: 378) zu verbinden. Brauchbar für die Implikationen bei der Anwendung von „Intersektionalität“ hinsichtlich der vorliegenden Studie sind vor allem die Verdienste für die empirische Umsetzbarkeit. Insbesondere Befunde, die für die Handhabung auf der Meso-Ebene sprächen, sind allerdings auch bei der Intersektionalität äußerst rar (vgl. ähnlich Tuider 2015: 173). Gehäuft finden sich neben biographischen Analysen (Degele/Winker 2009; Çetin 2012; Langsdorff 2012; Paulus 2012; Schrader 2013; Amirpur 2016) noch diskurstheoretische Ansätze (zum Beispiel Tuider 2013; Marx Ferree 2010; Kerchner 2011). Kubisch warf bereits 2008 die Frage auf, in „welcher Weise die ,matrix of domination“ auf Identität und Interaktion [wirkt] und wie die Akteure in ihrer Interaktion Strukturen von Unterdrückung aufrecht [erhalten]" (Kubisch 2008: 48). Etwas weiter gefasst lässt sich die Frage formulieren: In welcher Weise funktionieren Zuschreibungen und Herstellungspraxen sozialer Differenz ganz allgemein, ohne den Zusatz, dass damit „Unterdrückung“ hergestellt werden muss. Letztlich stellt Anthias fest, dass die eine Seite der Intersektionalitätsforschung zwar die mehrdimensionale Betrachtungsweise von Unterdrückung in den Blick nehme (zum Beispiel bei afroamerikanischen Arbeiter-Frauen in den USA). Die andere Frage danach ,to understand the processes" (Anthias 2005: 33) der mehrdimensionalen Ungleichheit, sei ,a far more difficult task“ (ebd.). Als Ausgangspunkt für die Forschungspraxis verstanden (Schultz 2011: 132 f.), geht zum einen das Konzept der Intersektionalität davon aus, dass soziale Ungleichheit auf Grundlage der „typischen Ungleichheitsachsen“ tatsächlich existiert. Intersektionalität soll ferner als heuristisches Modell verstanden werden (vgl. Knapp 2013: $344 \mathrm{f}$. in Anlehnung an Yuval-Davis 1992). Für das hier vorliegende Forschungsvorhaben besteht der Verdienst der Intersektionalität darin, Differenz und Ungleichheit gemeinsam zu denken (vgl. Knapp 2013: 343). Betrachtet man soziale Grenzmarkierung nicht, ,allein als Differenzkategorien, die durch die gesellschaftliche Lage der Einzelnen bestimmt wird, sondern berücksichtigt auch, wie einzelne Kategorien in die Konstruktion anderer Kategorien eingehen" (Schultz 2011: 133), ist Intersektionalität als Ansatz zu verstehen, der in Abhängigkeit zur Forschungsfrage jedes Mal neu konzipiert und kontextualisiert werden muss. So wird für die vorliegende Arbeit konstatiert, dass für die Analyse von Interviewdaten Intersektionalität als Ausgangspunkt für die symbolische Herstellungspraxis sozialer Differenzmerkmale in ihrer Gleichzeitigkeit und in ihrer Wechselseitigkeit von Bedeutung ist. Konkret ist daher zu fragen: Inwiefern beeinflussen sich verschiedene Differenzmerkmale 
gegenseitig, die sich aus dem Material extrahieren lassen? Inwiefern trifft dies auf verschiedene gesellschaftliche Ebenen zu bzw. werden diese in den Wechselbeziehungen durchkreuzt? Es wird also betrachtet, in welcher Weise soziale Differenzmerkmale sich gegenseitig verstärken, bedingen, nivellieren oder gegebenenfalls aufheben. Wird das heuristische Modell durch die Implikation erweitert, dass sich soziale Differenzmerkmale gegenseitig nivellieren können, also zum Beispiel durch eine privilegierte Klassenlage ein nachteiliger Migrationshintergrund abgeschwächt wird, kann das heuristische Modell der Intersektionalität über die Diskriminierten-Analyse hinausgehen. Der Nicht-Einbezug dieser Perspektive wurde von Meuser als Kritik an dem Konzept anführt (Meuser 2013b: 432).

\subsubsection{Anwendungsorientierter Ansatz: Vielfalt/Diversity}

Neben den bisher vorgestellten, konzeptionell ausgereifteren Ansätzen, die Dynamiken, Fallstricke und Forschungslücken der Herstellungsweise sozialer Differenz aufzeigen, bildet der Diversity-Ansatz eine eher anwendungsorientierte und in Teilen bewusst unscharfe Modellierung sozialer Differenz. Meuser (2016: 789) zeigt zwei Seiten der „Diversity-Medaille“ auf: Zum einen die makro-soziologische Deskription über plurale Gesellschaften und zum anderen Diversity als normatives Handlungsziel, was mit dieser Pluralität „zu tun“ sei. Gerade zweiteres wird in betriebswirtschaftlicher Ausrichtung auf der Meso-Ebene von Organisationen als eine ökonomisch gewinnbringende Maßnahme behandelt.

In erster Linie geht es bei Diversity darum, soziale Vielfalt zu benennen und einen Versuch zu skizzieren, mit ihr ,umzugehen“, und zwar mit der Leitidee, bei Vielfalt handele es sich um eine Humanressource, ein Potenzial, das es zu nutzen gelte. In dieser „Semantik einer wertgeschätzten Vielfalt, die den Fremden zu einem ,nützlichen Anderen' (Lederle 2008: 264) macht, [wird] der Differenz das Bedrohliche [genommen], als das sie nicht selten wahrgenommen wird“" (Meuser 2013b: 167). Konzeptionell nicht miteinander verbunden, aber doch entstehungsgeschichtlich hinsichtlich Modellierungen zu sozialer Differenz verwandt, gedieh „Diversity“ als begriffliches „Etikett“ (Knapp 2013: 343) parallel zum Topos der Intersektionalität (vgl. Knapp 2013: 343 f.). Parallel an beiden Konzepten ist der Umstand, dass sich das theoretische Konzept von der Ursprungsidee mittlerweile losgelöst hat und anderen Ansprüchen genügen möchte als vormals intendiert. So entstanden beide Ansätze aus einer Logik, die Diskriminierung und Benachteiligung von Minderheiten aufdecken und gegenübertreten sollten. Während „Intersektionalität“ weiterhin als Forschungsprogramm fungieren kann, verbleibt Diversity mit der Zugabe „Management“ in der Ambivalenz „ökonomische Profit- und eine gesellschaftliche 
Gemeinwohllogik [...] miteinander zu ,versöhnen““ (Meuser 2016: 792) zu sollen. Schließlich ,wird [inkludiert], wessen Humankapitalkonfiguration den sich wandelnden Organisationsanforderungen kompatibel“" (Meuser 2013: 172) erweisen.

Angesichts der sozialwissenschaftlichen kritischen Reflexion des Begriffs soll die betriebswirtschaftliche Sichtweise nicht vorenthalten werden. Aus der organisationalen Anwendungsperspektive (Meso-Ebene) entstand laut Sander (2006) die Idee, „Diversity“, durch den Wunsch, besondere Maßnahmen für Benachteiligte im Kontext ethnischer Divergenz in den USA zu schaffen. „Diversity“ umfasst primär unterschiedliche Diversitäten, also „Dimensionen“ von Andersartigkeiten (vgl. Sander 2006: 77). Einerseits handelt es sich bei den Ausführungen um Ansätze, die das Wort „Diversity“ in Dimensionen zu zerlegen versuchen und diskutieren, welche Arten von Andersartigkeit dazugehören oder dazugehören können. Sepehri (2002) setzt das Diversity-Management-Konzept ein, um in Anlehnung an Krell gegen das ,homogene Ideal" zu argumentieren und aufzuzeigen, dass es für eine Organisation eine gesunde Balance zwischen Vielfalt und Gemeinsamkeiten geben muss. Thomas und Ely (1996) betrachten Diversity als schillernden Begriff, der je nach historisch-politischem Kontext entweder als Anti-Diskriminierungsparadigma („Fairness \& Diskriminierungsansatz“) oder als legitime Leitidee für das ökonomische Ausschöpfen von Humanpotential zur Erschließung neuer Märkte und Zielgruppen („Marktzutritts- \& Legitimitätsansatz“) benutzt werde. Da beide schnell an ihre analytischen Grenzen stoßen, diskutieren die Autoren Diversity eher als ganzheitliches Konzept, das im Optimalfall sowohl der Ressourcengewinnung, als auch als moralisches Motiv verstanden und im „Lern- und Effektivitätsansatz" vereint wird. Diese Ambivalenz wird in den Sozialwissenschaften kontrovers diskutiert. Auf der Anwendungsebene in erster Linie privatwirtschaftlicher Institutionen werden diese „Dimensionen“ aufgefächert in Geschlecht, Ethnizität, Alter, Behinderung und sexuelle Identität (vgl. Salzbrunn 2013: 376, Charta der Vielfalt) ${ }^{15}$. Davon leitet sich das Gesamtkonzept „Managing Diversity“ ab, wobei die Art der Dimension das Konzept und die Strategie der Umsetzung maßgeblich beeinflussen. Insofern verspricht „Diversity Management“, zumindest in der Theorie, den Schritt zum ganzheitlichen, zusammenhängenden Konzept über den Umweg der „Einzeldiversitäten“ von Individuen. Stuber (2002) teilt seine gedanklichen Zugänge dagegen in personenbezogene und organisationsbezogene Perspektiven auf, die es ihm ermöglichen, die Bedeutung von Diversity auf der individuellen und auf der institutionellen Ebene greifbar zu machen. Die oben beschriebene Aufteilung von dem Konzept-Charakter und den „Vielfaltsdimensionen“ gelingen ihm durch die Erweiterung des Schemas mit den Kategorien „Sachebene“ und 
„Mentalebene“, da die Sachebene die tatsächlich erkannten Unterschiede und deren Nutzung durch die Organisation umfasst und die Mentalebene die hierfür notwendige „Haltung“ offenbart. Was genau mit „Haltung“ gemeint sein könnte und wie stark es formalisiert in Erscheinung treten soll, zum Beispiel in Leitlinien einer Organisation usw., bleibt allerdings offen. Wie sehr der Begriff schillert, beweist der Band „Diversity Outlooks“ von Koall/Bruchhagen/Höher (2007). Die Zugänge variieren zwischen einer vagen „DiversityPhilosophie“, über konkrete Management-Strategien in verschiedenen Handlungsfeldern bis zur Weiterführung vorhandener Theorieansätze zu sozialer Differenz (vgl. Koall/Bruchhagen/Höher 2007).

Diversity soll an dieser Stelle verstanden werden als jegliche Andersartigkeit und kann in allen erdenklichen Kombinationen vorkommen. Die prominent propagierten „Dimensionen“ sozialer Differenz sind Hilfsmittel, um das Konzept zu erschließen und entsprechende Maßnahmen zu implementieren, geben aber verkürzt gedacht einem Marketinginstrument ein schickes Gewand. Auf der gesellschaftlichen Bedeutungsdimension wird als übergeordnetes Ziel propagiert, eine „Geisteshaltung ,Offenheit““ (Stuber 2002: 15) zu schaffen. Verkürzt ist das Konzept dahingehend, als das es eine „Achse [...] der Ungleichheit“ (Aulenbacher u. a. 2007) völlig auslässt: Dass in Diversity-Ansätzen die Klassenlage nur am Rande mitgedacht wird, geht laut Salzbrunn einher mit einem Cultural Turn der Sozialwissenschaften (Salzbrunn 2012: 376). Da es sich bei Stiftungen um Organisationen handelt, die in die öffentliche Sphäre hineinwirken möchten, sollen sie darauf hin untersucht werden, ob sie Diversity (Management) explizit als Unternehmensstrategie erwähnen oder umgekehrt, Elemente, die sie ausmachen, als Diversity Management erkannt werden können. Gerade Bürgerstiftungen werden unter dem Aspekt diskutiert, sie besäßen Strahlkraft in lokalen bzw. kommunalen Handlungsfeldern (vgl. Becker/Wolf 1999; Wolf 2009) Auch Diversity wird vermehrt im Rahmen stadtsoziologischer Betrachtungen (Meuser 2013: 173 ff.; Salzbrunn 2012: 382 ff.; Meuser 2016: 792) hinzugezogen. Dies illustrieren zum Beispiel die Beiträge im Sammelband „Neue Vielfalt in der urbanen Stadtgesellschaft“ (Bukow/Heck/Schulze/Yıldız 2011).

\section{Zwischenfazit}

Die Ansätze, die unter 3.2 und 3.3 vorgestellt wurden, haben verdeutlicht, welche Aspekte bei der synoptischen Zusammenführung zu sozialer Differenz zu berücksichtigen sind und worauf sie sich stützen werden. Die Ansätze zeigen, dass Differenz-Merkmale zumeist singulär behandelt werden. Im Rahmen der sozialkonstruktivistischen Ansätze trifft dies auf das Merkmal „Geschlecht“ 
zu, in kulturwissenschaftlichen Ansätzen wird „Ethnizität“ ins Zentrum gerückt. Die Intersektionalitätsforschung arbeitet akribisch daran, Klassenlage und ethnische Zugehörigkeit eingebettet in solche des Geschlechts in ein wechselseitiges Modell zu überführen. Dabei soll die empirische Umsetzbarkeit gewährleistet werden, was in erster Linie mit Blick auf die Forschung zu individuellen Lebensverhältnissen gelingt. Der Diversity-Ansatz möchte soziale Vielfalt anwendungsorientiert verstehen, formuliert daraus eine gewinnbringende Management-Strategie und scheitert an dem Punkt, Klassismen mitzudenken. Eine Verknüpfung von verschiedenen gesellschaftlichen Ebenen gelingt bei der Grenzziehungsmetapher des Boundary Making, wo gesellschaftliche Verhältnisse und symbolische Zuschreibungen theoretisch synchronisiert werden. Dem Aufzeigen von Benennungspraxen selbst nimmt sich das Othering explizit an und unterstreicht die Hervorbringung eines „Anderen“ (,die') gegenüber einem „Eigenen” (,Wir $)$. Bei der Hybridität und Postmigration wird herausgestellt, dass Forschungsparadigmen selbst perspektivisch verengt sein können, da sie eben aus der Sicht der privilegiert wahrgenommenen Gruppe entwickelt worden sind. Diese Kritik schimmert bereits beim Intersektionalitätsparadigma deutlich durch. Der Ansatz der Postmigration plädiert dafür, die Forschungsparadigmen bezüglich Personen mit nicht-deutschen Vorfahren hinsichtlich ihrer kulturellen Angepasstheit hintanzustellen und stattdessen so komplex zu forschen, wie es für andere Differenzkategorien seit Jahrzehnten praktiziert wird. Überlegungen zur Entstehung von Klassismen, über die empirische Anwendbarkeit allgemein oder eine gesellschaftliche Verortung des Ansatzes hinsichtlich einer Meso-Ebene, finden sich hingegen nicht.

\subsection{Analyserahmen für soziale Differenz}

Im Zwischenfazit wurden die Stärken und die Schwachstellen der einschlägigen Ansätze, die sich vor dem Hintergrund der Fragestellung im engeren Sinne mit sozialer Differenz befassen, kurz angerissen. Es wurden Lücken aufgezeigt, die mit der synoptischen Zusammenstellung kompensiert werden sollen, wobei der Analyserahmen weiterhin als Heuristik zu verstehen ist. Ein kurzer Rückblick soll den weiter unten zu machenden Vorschlag aufklären.

Das vorliegende Kapitel hatte zum Ziel, einen Analyserahmen zur Herstellungsweise sozialer Differenz für einen organisationalen Dritte-Sektor-Akteur zu formulieren. Die Schwierigkeiten bei der Modellierung eines Konzepts ,,soziale Differenz" sind dabei vielfältig: Zum einen ist eine Spaltung der For- 
schungstraditionen in „Differenz-“ und in „Ungleichheitsforschung“ zu beobachten. Dies hat zur Folge, dass für eine Herstellungsanalyse sozialer Differenz die gemeinsame Betrachtung von gesellschaftlicher Benachteiligung und sozialem Gruppenmerkmal nicht ohne weiteres möglich ist. Zum anderen beschäftigen sich Theorien der Differenzforschung zumeist auf der Mikro-Ebene mit Interaktionen, Alltagspraxen und Biographien. Die Ansätze zu sozialer Ungleichheit nehmen eher makro-soziologische Zustände in den Blick. Hirschauer $(2014,2017)$ versucht, einen Ansatz zu entwickeln, der der Praxis von „Humandifferenzierung“ auf den Grund gehen möchte. Dieser beinhaltet allerdings neben ersten Überlegungen zur Triangulation der Ansätze auch den Versuch, die Relevanz von sozialen Kategorien in den jeweiligen Kontexten einschätzbar zu machen. Dieses Vorhaben geht für die vorliegende Studie über das Erwünschte hinaus, da nicht die Einschätzbarkeit einer bestimmten sozialen Kategorie für ein bestimmtes Handlungsfeld, sondern die Herstellungsweise allein im Vordergrund steht. Dennoch benennt Hirschauer das „Problem“, um das es hier geht, sehr genau:

Die Seite der kontingenten Herstellung einer Unterscheidung ist oft gut ausgeleuchtet, die Seite der Kontingenz im Gebrauch durch soziale Prozesse dagegen unterbelichtet (Hirschauer 2014: 181, Hervorhebung im Original).

Welche sozio-demographischen „Eigenschaften“ für einen höheren sozioökonomischen Status nachteilig sind (zum Beispiel sozial ,niedrige' Herkunft, weibliches Geschlecht, Migrationsstatus) ist, wie erwähnt, für die MakroEbene allgemein gut bekannt. Was diese allerdings in ihrer Herstellungsweise auszeichnet, ist seltener Gegenstand von wissenschaftlicher Forschung. Welche Erkenntnisse die vorgestellten Ansätze für den Analyserahmen bereithalten, soll mit Hilfe von Tabelle 2 visuell sortiert und im Folgenden genauer ausgeführt werden. 
Tabelle 2: Sortierung der relevanten Konzepte zu sozialer Differenz

\begin{tabular}{|c|c|c|}
\hline Ebene & Ansatz | Konzept (beispielhaft) & Quitessenz für Analyserahmen \\
\hline $\begin{array}{l}\text { Mikro-Ebene: } \\
\text { Fokus Interaktion }\end{array}$ & $\begin{array}{l}\text { - Un/doing difference } \\
\text { (Hirschauer 2014, 2017) } \\
\text { - Intersektionalität } \\
\text { (Degele/ Winker 2009, } \\
\text { Anthias 2005) } \\
\text { - "doing gender" } \\
\text { (West/ Zimmermann 1987); } \\
\text { "doing difference" } \\
\text { (West/Fenstermaker 1995) }\end{array}$ & $\begin{array}{l}\text { Verschränkung verschiedener } \\
\text { Differenzmerkmale bei Individu- } \\
\text { en, die Ungleichheit markieren; } \\
\text { Fokussierung von Herstellungs- } \\
\text { praxen, die Differenzmerkmalen } \\
\text { die immer gleiche Bedeutung in } \\
\text { einem bestimmten Handlungs- } \\
\text { kontext absprechen }\end{array}$ \\
\hline $\begin{array}{l}\text { Meso-Ebene: } \\
\text { Fokus Organisation }\end{array}$ & $\begin{array}{l}\text { - (Managing) Diversity } \\
\text { (Sepehri 2002, Stuber 2002, } \\
\text { Salzbrunn 2012, Meuser 2013) }\end{array}$ & $\begin{array}{l}\text { Wertschätzung hinsichtlich } \\
\text { und Profitmaximierung mit Hilfe } \\
\text { sozialer Vielfalt; kommunaler } \\
\text { Kontext }\end{array}$ \\
\hline $\begin{array}{l}\text { Makro-Ebene: } \\
\text { Fokus Herrschafts- } \\
\text { systeme }\end{array}$ & $\begin{array}{l}\text { - "Boundary making“ } \\
\text { (Wimmer 2008) } \\
\text { "Othering“ } \\
\text { (Castro Varela/ Dhawan 2005) } \\
\text { - Hybridität/ Postmigration } \\
\text { (Bhabba 1994, Hall 2004, Reckwitz } \\
\text { 2015, Foroutan 2017, Yildiz 2014) } \\
\text { - Intersektionalität (Crenshaw 1988, } \\
\text { Klinger 2008, Knapp 2013) }\end{array}$ & $\begin{array}{l}\text { Aufzeigen von sozialen und } \\
\text { symbolischen Grenzziehungen } \\
\text { zw. Menschengruppe; „Wir/ } \\
\text { Die - Differenzmarkierung; } \\
\text { Erweiterung kulturräumlicher } \\
\text { Betrachtungen jenseits des } \\
\text { ethnischen Fokus; Verschrän- } \\
\text { kung verschiedener Differenz- } \\
\text { merkmale bei Menschen- } \\
\text { gruppen, die Ungleichheit } \\
\text { markieren }\end{array}$ \\
\hline
\end{tabular}

(Quelle: eigene Darstellung)

Dem konstruktivistischen Paradigma folgend begann das Kapitel mit einer Skizze über sozialkonstruktivistische Ansätze, die allesamt von der „Gemachtheit" der Merkmalszuschreibungen ausgehen. Diese gilt es demnach zu rekonstruieren, wobei eingebettet in größere gesellschaftliche Kontexte sich die Fragen nach Macht und Hierarchie aufdrängen. Der Doing-difference-Ansatz von West/Fenstermaker (1995) in der gleichfalls sozialkonstruktivistischen Theorientradition bezieht sich auf einer Mikro-Sicht auf routinisierte Alltagspraxen und unterstützt die Prämisse, dass soziale Differenz in irgendeiner Weise ein soziales Produkt sein muss. Forscherinnen und Forscher bemühen sich darum, die Verschränkung relevanter Differenzmerkmale zu konzeptualisieren (siehe Tabelle 2) und den Differenzmerkmalen ,Rollen` zuzuweisen. Zusätzlich machen sie auf den Aspekt des ,Widerhalls' einer Konnotation aufmerksam: Die Hervorbringung von sozialer Differenz findet nämlich erst dann statt, wenn sie 
einen gewissen Grad an allgemeiner Verbreitung erlangt. Hier gilt es zu erfassen, inwiefern die öffentliche Sphäre hinsichtlich der Differenzbenennung in den Blick zu nehmen ist.

Beim Boundary-making-Ansatz unterscheiden Forscherinnen und Forscher die Ebene der sozialen Ungleichheit und die symbolische Grenze der Benennungspraxis sozialer Differenz (siehe Tabelle 2). Die These lautet, dass diese in Wechselwirkung zueinanderstehen. Dabei ist der Vorschlag bemerkenswert, nicht nur die Grenzmarkierung an sich zu untersuchen, sondern auch die Beziehung der ,Benenner' gegenüber den ,Benannten' in den Fokus zu rücken. So stellt sich die Frage, wie in diesem Kontext Aussagen von Repräsentantinnen und Repräsentanten privilegierter Organisationen, wie es Stiftungen sind, zu werten sind, die die Hervorbringung sozialer Grenzziehungen in Form symbolischer Zuschreibungen vornehmen. Besonders interessant gestaltet sich dieser Punkt darin, als dass Stiftungen als eigentlich private Akteure einer selbstverpflichteten Gemeinwohlorientierung nachgehen.

Mit Blick auf Ansätze, die ursprünglich den Postcolonial Studies zugerechnet werden, fungiert das Othering-Konzept als deutliche Kritik an der Herstellungspraxis von Kollektivismen, bei der die Hervorbringung eines Eigenen und Fremden thematisiert wird (Tabelle 2). Bei der Hybridität geht es dann darum, dass in den jeweiligen Kontexten als selbstverständliche Trennlinie markierte Differenz eigentlich keine homogenen kulturellen Räume voneinander trennt. Geht man davon aus, dass kulturelle Räume eigentlich überlappen, ist bei hybriden Identitäten, beispielsweise ethnischen Minderheiten, von einer ganz eigenen heterogenen Symbiose als Lebenswirklichkeit auszugehen. Die daran andockenden deutschen Postmigrationsforschenden (Foroutan 2016; Y1ld1z 2014) stellen dabei die unumgehbare Notwendigkeit in Frage, Migrationsforschung dogmatisch als Integrationsforschung zu begreifen, ohne sich vom Migrationsnarrativ gänzlich zu verabschieden. Sie bieten ein Forschungsprogramm an, in dem die Koordinaten zur Verortung von Minoritäten mehrdeutig erfasst werden können, wie es wahrscheinlich praxisbezogen in sozialen Projekten des Dritten Sektors eher vorzufinden sein wird. Diese Ansätze beinhalten Kritik, die auch im Rahmen der Intersektionalitätsforschung zum Tragen kommt. Hier wurde ursprünglich durch Afroamerikanerinnen der Vorwurf genannt, ,klassische' Feministinnen sprächen nur für , weiße' Frauen. Besonders hervorzuheben ist daher die Intersektionalität für ihre Verdienste bei der Verschränkung von Differenzmerkmalen, die anders als beim Doing-difference-Ansatz (3.2.2) an dieser Stelle vordergründig die Makro-Ebene sozialer Ungleichheiten erfasst (Tabelle 2). Auch der Diversity-Ansatz entwickelt sich größtenteils von seiner Entstehungsgeschichte als Gleichstellungsinstrument in Richtung Human-Resources-Verwertung weg. Daher wird hier davon ausgegangen, dass von Seiten der Akteure, die mit Deutungshoheit ausgestattet 
sind, darauf geachtet wird, wie Verschiedenartigkeiten von in erster Linie Arbeitnehmenden (oder im Falle von Stiftungen: von Zielgruppen und Kooperationspartnern) auf der Meso-Ebene der Organisationen nützlich gemacht werden können (Tabelle 2) - wobei Meuser (2013) und Salzbrunn (2013) folgend der Nutzen erstrangig, der ethische Aspekt der Inklusion zweitrangig ist. Auf einer übergeordneten Konzeptebene soll das Paradigma als Aushängeschild einer bestimmten Weltanschauung hinsichtlich Menschengruppen fungieren, bei der, Vielfalt' akzeptiert und Unterschiedlichkeiten gegenüber , offen ' begegnet werden solle.

Nun wird deutlich, welche Schwerpunkte sich beim Umgang mit der Forschungsfrage ergeben.

Erstens sind verschiedene Ungleichheitsdimensionen in der Analyse ihrer potenziellen Bedeutung für die Benennungspraxis gleichwertig zu behandeln (Geschlecht, Ethnizität, Klasse etc.). Diesen Makel, soziale Differenz stets über eine Art Leitdifferenz zu erfassen, weisen fast alle oben vorgestellten Ansätze auf, auch wenn sie versuchen, unterschiedliche Differenzmerkmale konzeptionell zusammenzufügen. So sind die sozialkonstruktivistischen Ansätze, die auf soziale Differenzmerkmale angewendet werden, ausgehend von der Geschlechterforschung entstanden und im Laufe der Zeit auf andere Differenzen (ethnische Zugehörigkeit, Klassenlage) erweitert worden (s. Un/doing-difference-Ansatz; Hirschauer 2017; West/Fenstermaker 1995). Die Forschungsansätze der Postcolonial Studies fokussieren Ethnizität, die Intersektionalitätsforschung widmet sich, wie oben erwähnt, hauptsächlich der Verschränkung von Ethnizität und Geschlecht, um hieran Überlegungen zur Klassenlage anzuschließen. Das Diversity-Konzept ist anders gelagert, da es eher in der praktischen Umsetzung verschiedener Zielvorhaben zur Maximierung von Profit herhalten muss. Dabei werden Klassismen unberücksichtigt gelassen. Allein die Grenzziehungmetapher des Boundary-making-Ansatzes kann diesem Anspruch genügen, indem sie soziale Hybridität als Zusammenspiel beliebiger „,kultureller Codes“ ausweist. Allerdings verweilt sie dafür auf einer sehr abstrakten, für die empirische Forschung schwer zugänglichen Ebene. Für die hier interessierende Forschungsperspektive soll daher gefolgert werden, dass die Durchsicht der empirischen Daten nicht über eine dominante „Leitdifferenz“ erfolgen soll. Im Umkehrschluss ist zu schauen, ob beispielsweise Othering nicht auch im Kontext von Klassismen ebenso vorkommt wie bei der Diskriminierung ethnischer Minderheiten. Kulturelle Codes werden, wie im Boundary-making-Ansatz dargelegt, nicht nur im ethnischen Kontext verstanden und ein doing difference kann körperliche Beeinträchtigungen in Zusammenhang mit Alter genauso fokussieren wie Geschlecht und Ethnizität.

Zweitens soll die gesellschaftliche Positionierung bzw. das inszenierte Verhältnis von „Benennern“ und „Benannten“ fokussiert werden, da vor allem 
Theorien wie jene zur Hybridität oder Intersektionalität, die aus einem emanzipatorischen Ansinnen entstanden sind, dieses Verhältnis als Machtasymmetrie thematisieren. Doch ist das bei Stiftungsprojekten mit einer zivilgesellschaftlichen Verortung keine triviale Aufgabe, da sie bekanntlich nicht nur soziale Ungleichheit angehen möchten, sondern auch durch Personenauswahl (zum Beispiel für Stipendien) Exklusivität schaffen. Potenzielle Zielgruppen werden also nicht nur aus einer Fürsorge-Absicht heraus ausgewählt, sondern ggf. umworben. Daher wird der Vorschlag gemacht, dass die Selbstdarstellungen der Repräsentantinnen und Repräsentanten genau zu fokussieren sind, um in Einbettung ihrer gesellschaftlichen Rolle auch die Beziehungsherstellung zu verschiedenen gesellschaftlichen Gruppen erörtern zu können.

Unter drittens werden zwei verschiedene Aspekte gleichzeitig in den Blick genommen, da sie schwer voneinander zu trennen sind: die Benennungspraxis hinsichtlich der Konnotation einer sozialen Differenzkategorie sowie die Wechselbeziehungen zwischen den Differenzmerkmalen. Bei der Betrachtung der Wechselbeziehungen zwischen den Differenzmerkmalen soll der Anschluss an das Un/doing-Konzept und an die Intersektionalitätsforschung gesucht werden. Sie zeigen auf, dass soziale Differenzkategorien sich wechselseitig bedingen können, aufheben oder verstärken. Selbstverständlich gilt es auch zu betrachten, inwiefern soziale Differenz lediglich (re)produziert wird. Im Zusammenhang mit der Benennungspraxis sollen soziale Differenzmerkmale von der Negierung, über die Relativierung bis hin zur Aufwertung begutachtet werden, und zwar aufgefächert auf die verschiedenen sozialen Merkmale, die im empirischen Material auftreten. Verschiedenartigkeiten, die soziale Ungleichheit mitproduzieren, sind so in ihrer Wechselbeziehung im Zusammenspiel ihrer Konnotationen analysierbar. Bestehende Forschungsparadigmen insbesondere der klassischen Migrations- bzw. Integrationsforschung sind auf diese Weise automatisch ,entdichotomisiert' (etwa: einheimisch/ausländisch). Einhergehend mit der Analyse der Wechselbeziehungen zwischen den Differenzkategorien ist zudem zu erörtern, welche Konnotationen im Zusammenhang mit welchen Differenzkategorien besonders häufig bzw. besonders selten vorkommen und welche Häufungen sich bei der Wirkebene (Mikroebene: Individuen; Mesoebene: Netzwerke \& Organisationen; Makroebene: gesellschaftliche Ebene) des konkreten Vorhabens erkennen lassen. Von besonderem Interesse wird es sein, ob sich ein Muster erkennen lässt, das Aufschluss gibt, inwiefern Differenzmerkmale, Wirkebene und Konnotationsmodus zueinanderstehen.

„Zu fragen wie erfordert oft, daß wir eine radikal naive Haltung einnehmen und das Offensichtliche zum Problematischen machen" (Knorr-Cetina 1984: 49, Hervorhebung im Original). Die Vorstellung der verschiedenen An- 
sätze, gerade auch derjenigen, die ihren Forschungsansatz als Kritik an bestehenden Paradigmen formulieren, hat zudem gezeigt, dass es selbst auf der Ebene der theoretischen Modellierungen zur Analyse sozialer Differenz eine perspektivische Verengung gibt, die Salzbrunn (2012) wie folgt auf den Punkt bringt: „Wenn [...] Minderheiten benannt werden, geschieht dies zwangsläufig von der Warte der Mehrheit aus - die für sich die Definitionsmacht des Normalen und des Normabweichenden beansprucht" (Salzbrunn 2012: 379). Im Rahmen der Migrationsforschung traf dies einerseits jahrzehntelang zu. Hierzu muss ergänzt werden, dass dieser Vorgang über den (politischen) Anspruch der ,Mehrheitsperspektive“ hinaus auch Resultat unbewusst getätigter Differenzbenennungspraxen sein kann bzw. ist. Die perspektivische Verengung resultiert gegebenenfalls auch daraus, dass meist eine Kongruenz zwischen biographischen Prägungen der Autorenschaft und dem Untersuchungssubjekt existiert oder vorausgesetzt wird bzw. sogar zum „Rechtfertigungsdruck“ (Knapp 2013: 346 mit Verweis auf Meuser 2010) kommt, wenn dem nicht so ist; wenn also Männer zu Geschlechterverhältnissen forschen, Heterosexuelle zu queeren Themen oder ausländische Wissenschaftlerinnen nicht zu Migrationsfragen uvm. Wenn in der empirischen Analyse Führungskräfte in den Stiftungen in einer Weise zu den sozialen Projekten befragt werden, als sei es nicht rational ersichtlich, warum Vorlesepatenschaften mit Kindern aus prekären Elternhäusern initiiert werden oder warum Studierende mit Migrationshintergrund ein eigenes Stipendienprogramm auferlegt bekommen, kann dies nur mit einem theoretischen Modell gelingen, dass ebenfalls eine „Attitüde der künstlichen Dummheit“ (Hitzler 1986) bzw. eine Art „Grundnaivität“ aufweist. ${ }^{16}$ Mit der Erarbeitung dieser Forschungsansätze, die dieser Perspektivierung mal mehr, mal weniger unterliegen, und ihrer Triangulation, soll diesem Anspruch genügt werden.

16 Hitzler bezieht dies in erster Linie auf die empirische Arbeit. An dieser Stelle soll es auf die theoretische Forschungsperspektive ausgeweitet werden. 


\section{Stiftungen und soziale Differenz}

\subsection{Methodisches Design für die Auswertung}

Zur Erhebungsmethode des Experteninterviews haben sich in den letzten Jahren verschiedene Positionen manifestiert (vgl. Bogner/Menz 2009a). In ihrem Aufsatz „Das theoriegenerierende Experteninterview“ führen Alexander Bogner und Wolfgang Menz (2009b) eingangs aus, welche wissenschaftlichen Kontroversen zum Experteninterview bestehen. Sie beschreiben drei Typen von Experteninterviews (ebd.: 63 ff.). Zunächst folgt die Einordnung als Exploration (1), bei der das Experteninterview kein vollständiges Erhebungsinstrument einer Studie ist, sondern nur als eine Art Vorbereitungsinstrument für weitere Erhebungen dienen kann. Das systematisierende Experteninterview (2) begreift den Experten ,in erster Linie als ,Ratgeber' oder Inhaber von spezifischen gültigen Kenntnissen“"(Bogner/Menz 2009b: 65). Es geht konkret um die unmittelbaren Informationen, die Interviewpersonen als Trägerinnen und Träger einer bestimmten professionellen Rolle heraus aussprechen, also um das „,reflexiv verfügbare und spontan kommunizierbare [...] Handlungs- und Erfahrungswissen" (Bogner/Menz 2009b: 64), das in diesem Kontext relativ exklusiv ist. Dabei steht die Information selbst im Vordergrund, denn: „Nicht die Experten selbst sind aus dieser methodologischen Perspektive die Gegenstände der Untersuchung; diese fungieren vielmehr als Informanten in Bezug auf ihre eigentlichen Forschungsobjekte" (Bogner/Menz 2009b: 65). Um dies mit Mannheims Sinnebenen zu illustrieren (1964) geht es bei den Inhalten des systematisierenden Experteninterviews um den objektiven Ausdruckssinn und darum, wie die gewonnenen Informationen das Untersuchungsfeld strukturieren. Dieser Ansatz unterscheidet sich von der dritten Kategorie des Experteninterviews nach Bogner/Menz, die sie angelehnt an die Arbeiten von Meuser/Nagel (1991, 1995, 2005, 2009) als theoriegenerierend (3) beschreiben und die an wissenssoziologische Traditionen anknüpft.

Das theoriegenerierende Interview zielt im Wesentlichen auf die kommunikative Erschließung und analytische Rekonstruktion der „subjektiven Dimension“ des Expertenwissens. Subjektive Handlungsorientierungen und implizite Entscheidungsmaximen der Experten aus einem bestimmten fachlichen Funktionsbereich bezeichnen hier den Ausgangspunkt der Theoriebildung (Bogner/Menz 2009b: 66). 
Während also der systematisierende Ansatz auf das reflexiv verfügbare Wissen des Experten setzt, steht bei der theoriegenerienden Herangehensweise die Rekonstruktion des präreflexiv verfügbaren Wissensbestands im Vordergrund, was Rückschlüsse auf implizit kommunizierte Informationen, informelle Arbeitsabläufe und soziale Praktiken erlaubt. So führen Meuser/Nagel aus, dass das „Handeln von Experten [...] im hohen Maße von Relevanzen geprägt [ist], die den Experten nicht oder nur teilweise reflexiv verfügbar sind. Nicht nur Alltagswissen, auch Expertenwissen umfasst vortheoretisches Erfahrungswissen“ (Meuser/Nagel 2009: 470). Im Rahmen dieser Studie soll an die Herangehensweise von Meuser/Nagel angeknüpft werden. Dabei sollen zwar vorrangig Führungskräfte interviewt werden, allerdings muss gleichzeitig bei der Auswahl der Interviewpersonen darauf geachtet werden, dass die Expertinnen und Experten dennoch den ,Geschäftsbetrieb“ gut kennen. „Denn häufig wird die Bereitschaft und die Fähigkeit, für den Forschungsprozess relevante Informationen zu geben, bei Repräsentanten höherer Hierarchieebenen überschätzt", argumentiert Kaiser (2014: 132) und zeigt Beispiele aus der politikwissenschaftlichen Forschung, die die Befragung von Ministern und Staatssekretären thematisiert. Diese hohen Positionen zeichnen sich dadurch aus, dass die Funktionsträgerinnen und -träger viele Arbeiten an Mitarbeitende delegieren und vermutet werden muss, dass sie tatsächliche Arbeitsabläufe nicht nachvollziehen können. Daher werden für die vorliegende Studie Interviewpersonen auch dahingehend bewertet, ob sie im Rahmen ihrer beruflichen Tätigkeiten eine Balance zwischen der alltagsnahen operativen Ebene und der verantwortungsintensiven Top-Management-Ebene halten.

Auch die unscheinbare Interviewsituation einer wissenschaftlichen Befragung beinhaltet mehrere Wahrnehmungsebenen, die über die reine Informationsübermittlung zwischen fragender und befragter Person hinausgehen. Dazu gehört die mögliche Wahrnehmung der Interviewten, die sie gegenüber einer interviewenden Person durch verbalisierte und nicht-verbalisierte Informationen pflegen. Um die Analyse zur „Statusrelation“ (Meuser/Nagel 2009: 475) zu schärfen, stellen Bogner/Menz (2009b: 77 ff.) sechs Typen der Interviewerwahrnehmung vor, die auf die Interviews angewendet werden sollen. Zum einen beschreiben sie den Typus des „Interviewers als Co-Experten“ (1), bei der die interviewende Person als in der besprochenen Thematik kompetent und unhinterfragt mit selbst geteiltem Deutungswissen ausgestattet (vgl. ebd.) wahrgenommen wird und eine stärker ,horizontal ausgerichtete Kommunikationsstruktur“ (Bogner/Menz 2009b: 78) zu erkennen ist. Als zweiter Typus mit ähnlichen Auswirkungen auf die Interviewsituation wird der bzw. die Interviewende als „Experte einer anderen Wissenskultur“ (2) (vgl. Bogner/Menz 2009b: 79) umschrieben. Bei dieser Wahrnehmung gilt die Interviewperson 
zwar auch als kompetent, jedoch im Rahmen einer anderen Profession ähnlichen Ansehens. Als dritter Typus ist der „Interviewer als Laie“ (3) (vgl. Bogner/Menz 2009b: 82) zu nennen. Hier gilt es, die Ambivalenz für die Gesprächssituation im Auge zu behalten, da sich nach Abels/Behrens (2009) durchaus Vorteile ableiten lassen, die für die Informationsgewinnung zuträglich sind, wenn zum Beispiel die wahrgenommene Laienhaftigkeit seitens der Interviewperson dazu genutzt wird, besonders anschauliche und detaillierte Ausführungen zu animieren. Dagegen ist die Kategorie „Interviewer als Autorität“ (4) (vgl. Bogner/Menz 2009b: 84) sowie „Interviewer als potenzieller Kritiker" (5) (vgl. Bogner/Menz 2009b: 85) eher hinderlich für das Gewinnen ,guter' Interviewdaten, da davon auszugehen ist, dass in diesen Fällen die Interviewpersonen gehemmt sind. Bei der Wahrnehmung des „Interviewers als Komplizen“ (6) vermutet die Interviewperson ähnliche normative Orientierungen und gibt vertrauliche Informationen preis (vgl. Bogner/Menz 2009b: 86 f.). Diese Kategorisierung sowie die oben antizipierte „Geschlechter-“ bzw. „Statusrelation" sollen in diesem Abschnitt einfließen.

Hiernach wird betrachtet, was es für die vorliegende Arbeit und die Erhebung der insgesamt zehn Fälle mit elf Interviewpersonen bedeutet, wenn die Interviewpersonen stärker eine Nähe zur operativen Ebene oder stärker zur Top-Management-Ebene haben. Gesetzt den Fall, dass die Durchführbarkeit einer Rekonstruktion nach Kategorie drei (3) des Expertenwissens von der tatsächlichen formellen wie informellen Position sowie der individuellen Positionierung des jeweiligen Experten bzw. der jeweiligen Expertin in der einzelnen Stiftung abhängt, müsste streng genommen erst nach der Durchführung des Interviews die Bewertung erfolgen, ob die Interviewdaten als solche genutzt werden können oder ob dieser Fall nicht ,nur' für eine systematisierende Herangehensweise nach Kategorie zwei (2) verwendet werden kann.

2009 stellten Winker/Degele in ihrem Band „Intersektionalität. Zur Analyse sozialer Ungleichheit" eine Methodik vor, mit der vornehmlich narrative Interviews im Rahmen von Biographiestudien auf unterschiedlichen Ebenen intersektional ausgewertet werden können. Die Autorinnen weisen darauf hin, dass die verschiedenen Ebenen in den narrativen Interviews über soziale Praxen miteinander verknüpft sind (vgl. Winker/Degele 2009: 63 ff.). Angelehnt an Ausführungen von Bourdieu und Wacquant stellen die Autorinnen fest, dass das „bedeutet, dass sich keine soziale Tatsache aus ihrem singulären Sosein erklären lässt, jedes Element wird vielmehr durch die Beziehungen zu anderen Elementen charakterisiert" (Winker/Degele 2009: 64). Das zyklisch zu wiederholende Vorgehen besteht jeweils fallintern und fallübergreifend aus der Beschreibung der Identitätskonstruktionen und der Identifikation von symbolischen Repräsentationen (vgl. Winker/Degele 2009: 79 ff.) Neben diesen beiden Perspektiven bilden die Bezüge zu Sozialstrukturen und deren kontextuelle 
Analyse den dritten (bzw. fallübergreifend den sechsten) Schritt der Methode (vgl. ebd.). Zum Schluss werden die Wechselwirkungen der Ebenen in den Blick genommen.

Da im vorliegenden Fall Experten mit einem Leitfaden befragt wurden und nicht Individuen zu ihrem Lebenslauf, wurde eine starke Anpassung an das bestehende Forschungsvorhaben vorgenommen. Um mögliche Wechselwirkungen in den Argumentationskonstellationen hervorzuheben, soll von einem besonderen Fall ausgehend untersucht werden. Dabei wird nicht zwischen den trimensionalen Ebenen der Struktur, Repräsentation und Identität (vgl. Winker/Degele 2009: 68 ff.) unterschieden. Die Repräsentations- und die Identitätsebene werden bei den organisationalen Akteuren zusammengedacht, da die persönliche, aus der eigenen Biographie entstammende Identität beim Experten bzw. der Expertin als Vertreter/in einer Organisation eine untergeordnete Rolle spielt. Angelehnt auch an konventionelle Analysemethoden der qualitativen Sozialforschung werden die Wechselwirkungen zwischen den erwähnten Sozialstrukturen und der symbolischen Ebene aufgezeigt. Das bedeutet, dass eine implizite oder explizite Bewertung von Sozialstrukturen samt Erzählkontext genauer betrachtet wird. Auf diese Weise sollen intersektionale Deutungen möglich gemacht werden.

\subsection{Rahmendaten der Erhebung}

Die Akquise von Interviewpartnerinnen und -partnern war im Rahmen des Forschungsvorhabens nicht immer einfach. Der Umstand, dass die Interviewten nicht als Privatpersonen befragt wurden, sondern als Vertreterinnen und Vertreter einer Organisation, ließ das Interview scheinbar brisant wirken. Fast alle gaben an, dass Interview durch Vorgesetzte ,abgesegnet' bekommen zu haben bzw. es mit diversen Rechts- und Öffentlichkeitsarbeitsabteilungen eng abgestimmt zu haben.

Für die Rekrutierung der zehn Interviews wurden im Zeitraum August 2011 bis September 2012 zeitlich versetzt 21 Stiftungen angeschrieben. Zu zwei Stiftungen gab es persönliche Kontakte, ein weiterer Kontakt wurde über eine Kollegin hergestellt. Dieser eröffnete den Kontakt zu einer weiteren wichtigen Stiftung. Alle anderen wurden ohne vorherige Bekanntschaften akquiriert. Sie wurden auf unterschiedlichem Wege ermittelt. Zunächst war es wichtig, dass die Stiftung allgemein bekannt ist und sich als gesellschaftspolitische Akteurin engagiert. Des Weiteren wurden nur solche Stiftungen in die engere Wahl einbezogen, die erkennbar über ein relativ großes Stiftungsvermögen verfügen, 
was sich zum Beispiel bei der Anzahl der hauptamtlichen Beschäftigten niederschlägt oder auch darin, inwieweit die Stiftung über eine professionelle Infrastruktur verfügt (Stiftungsräume, Abteilungen mit unterschiedlichen Zuständigkeiten usw.). Bei zwei Stiftungen stellte sich heraus, dass sie lediglich als Zuwendungsempfängerinnen von Drittmitteln arbeiten, die zweckgebunden sind, und daher nicht in die Definition von Stiftungen passen, die der Studie zu Grunde gelegt wurde, also als autonom agierende Organisationen der Zivilgesellschaft. Sieben sagten ab, weil sie zu geringe zeitliche und personelle Ressourcen für ein Experteninterview angaben. Zwei Anfragen blieben unbeantwortet. Zwei Expertinnen wollten sich auch nach persönlichem Kontakt nicht für ein Interview zur Verfügung stellen. Beide Absagen erfolgten durch Frauen in Führungspositionen der operativen Ebene, die aufgrund einiger Eckdaten in ihren veröffentlichten Lebensläufen im mittleren Alter verortet werden können (ca. 40-55 Jahre) und eine sogenannte Man- bzw. Woman-in-the-middle-Position (Hill 1973) in der Organisation einnehmen. Als solche werden Tätigkeitszuschnitte in Organisationen bezeichnet, mit denen sowohl hohe Verantwortlichkeiten im Bereich Personal und Budget gegenüber Mitarbeitenden in gleichzeitiger hierarchischer Unterordnung gegenüber dem Top-Management verbunden sind.

Zwischen August 2011 und Oktober 2012 wurden zehn Experteninterviews mit elf Führungskräften aus Stiftungen durchgeführt, die allesamt zu den größten operativen Stiftungen der Bundesrepublik gehören, die sich im Bereich der sozialen Fürsorge, politischen Bildung und/oder gesellschaftspolitischer Interventionsmaßnahmen engagieren. Es wurden also jeweils Stiftungen ausgewählt, die sich in gesellschaftlichen Themenbereichen engagieren und somit diejenigen, die zum Beispiel stärker im Bereich Denkmalschutz usw. tätig sind, ausgespart.

Von den elf interviewten Personen waren vier Vertreterinnen und Vertreter einer partei- bzw. verbändenahen Stiftung, fünf waren aus einer unternehmensnahen Stiftung und bei einem Interview, dass mit zwei Personen gleichzeitig stattfand, handelte es sich um eine kommunale Stiftung. Der Stiftungssitz befand sich jeweils in einer mittelgroßen, großen bis sehr großen deutschen Stadt in verschiedenen Bundesländern. Die Interviewdauer reichte von ca. 40 bis 90 Minuten (jeweils reine Aufnahmedauer) und fand immer in den Räumlichkeiten der Stiftung selbst statt. Meist hatten die Gesprächspartnerinnen und -partner eigens einen Besprechungsraum organisiert, in dem die Tonaufnahme besonders gut möglich war.

Alle zehn Interviews sind der Kategorie d) des Gelingens nach Meuser/Nagel 1991 zuzuordnen, es besteht gute Verständigung, Einleitung ist nachvollziehbar, Neugierde und Interesse liegen auf beiden Seiten. Die Einstiegsfrage ${ }^{17}$

„Was sind Ihre Aufgaben hier in der Stiftung?“ 
zielte darauf $a b$, den Interviewten besser kennenzulernen und seine Position bzw. Aufgaben innerhalb der Stiftung herauszustellen. Zum einen war es so möglich, mit der interviewten Person ins Gespräch zu kommen, weil es eine leicht zu beantwortende Frage sein sollte. Zum anderen war es interessant in Erfahrung zu bringen, wer als Interviewpartner bzw. Interviewpartnerin genau vor einem saß, da Beschreibungen auf der Webseite über formale Positionen die konkreten Tätigkeiten in der Organisation nicht zwingend konkret wiedergeben.

Keine der Interviewpersonen hat die Aufzeichnung des Gesprächs verweigert, sodass alle zehn Interviews aufgezeichnet und transkribiert werden konnten. Der verwendete Leitfaden sah drei thematische Hauptbereiche vor, um das Agieren der Stiftung aus der Perspektive einer Führungskraft zu erfassen.

Nach der eingangs gestellten Frage zum Tätigkeitsprofil, die auch als ,Eisbrecher' bzw. zur Eingewöhnung in die Interviewsituation dienen sollte, folgten Fragen zum Leitbild oder zur Organisationskultur. Wörtlich wurde beispielsweise gefragt: „Gibt es ein Leitbild Ihrer Stiftung? Wenn ja, was ist Ihnen daran besonders wichtig?“ Daran anschließend: „Gibt es Projekte/Förderpraxen, die Sie besonders hervorheben möchten?" Dieser Block führte bei einigen Befragten zunächst zu Irritationen, da sie davon ausgingen, vorliegende Broschüren und die Webpräsenz der Organisation würden genügend Informationen liefern. Sie nahmen zum Teil Broschüren zur Darstellung der Projekte und anderer Vorhaben zur Hilfe, um die Tätigkeiten zu illustrieren. Wichtig war es an dieser Stelle aber herauszufinden, was die befragten Personen selber favorisieren und betonen. Letztlich haben sich alle Befragten auf die Fragen eingelassen und auch aus ihrer eigenen Perspektive berichtet.

Den zweiten Block des Leitfadens bildeten Fragen zur konkreten Stiftungsoperation und zur Felderfahrung. Hier sollte seitens der Befragten dargelegt werden, welche Programme, Projekte und sonstigen Vorhaben angestoßen worden sind und wie die operative Umsetzung wahrgenommen wurde. Im Leitfaden wurde hierfür die Frage verwendet: „Welche Projekte/Programme/Vorhaben verantworten Sie?“" um anschließend mit Details zu diesem Projekt fortfahren zu können. In einigen Fällen wirkten die Interviewten bereits sehr lange in der Stiftung mit oder waren sogar in die Gründung involviert. Hier wurde die „Urknall-Frage“ ergänzt: „Wenn wir die Entstehung der Stiftung mit dem Urknall vergleichen, was war der Anlass, dass die Stiftung gegründet wurde bzw. dieses Programm/Projekt ins Leben gerufen wurde?" Oder einfach nur: „Welche Idee stand zu Beginn hinter diesem Vorhaben?“ Ferner wurde, falls nicht von den Interviewten selbst erwähnt, nach der Praxiserfahrung gefragt: „Haben Sie Gegenwind bei der Umsetzung gespürt?“ Oder: „Was war beson- 
ders einfach/schwierig bei der Umsetzung des Projekts?" Somit war es möglich, Einblick in die Selbstwahrnehmung über das gesellschaftliche Wirken zu erhalten.

Damit zusammenhängend folgte der dritte Block über die Stiftungswirkung und die Zielgruppen. Die Interviewpersonen wurden gefragt, welche Wirkung sie sich von den Vorhaben erhofft haben und welche Gründe dafür sprachen, genau diese Projekte mit diesen Zielgruppen oder thematischen Ausrichtungen durchzuführen. Sie wurden gefragt: „Welche Wirkung erhoffen Sie sich von den Projekten?" Falls dieser Impuls nicht zur gewünschten längeren Narration führte, wurde nachgehakt: „Welchen gesellschaftlichen Beitrag leistet Ihrer Meinung nach das Projekt?" Zum Schluss wurde den Interviewten die Möglichkeit gegeben, falls nicht schon geschehen, eigene, als relevant wahrgenommene Punkte einzubringen. Dies wurde in einem Fall in Anspruch genommen.

Alle zehn Interviewpersonen waren das längere Reden bzw. Erzählen aus ihrem beruflichen Kontexten gewöhnt. Tatsächlich fabrizierten die meisten auf die jeweiligen Fragen kleine „geschlossene Reden“ (Gläser/Laudel 2010: 181), die in sich eine logische Abfolge besaßen. Da die Akquise der Interviewpartnerinnen und -partner nicht immer einfach war, muss entsprechend vermutet werden, dass die Interviewten zu Beginn zuversichtlich gestimmt werden mussten, dass ihre Daten nicht missbraucht werden würden und sie anfangs versucht haben, eine gewisse ,etiquette“ (Gordon 1975: 112) zu wahren. Direkt nach dem zweiten Interview wurde daher eine Verschwiegenheitserklärung vorbereitet und unterschrieben übergeben, damit die Interviewpersonen den verantwortungsbewussten Umgang mit den Informationen schriftlich dokumentiert erhielten. Das bewährte sich, sodass eine oberflächliche Beschreibung des Forschungsvorhabens und die vorab unterschrieben übergebene Erklärung die nachfolgenden Akquiseaktivitäten und Interviewsituationen entspannten. Auch eine gute Vorbereitung für einen Auftritt als kompetente Interviewerin tat ihr übriges (vgl. Gordon 1975: 112 ff.), um über die Expertinnen und Experten gute Daten zum Stiftungswirken zu gewinnen.

Dies gilt vor allem für den Zeitpunkt der Interviewsituation selbst, in der ein Gesprächspartner kaum relevante Informationen preisgeben wird, wenn er den Eindruck hat, dass der Interviewer selbst keine ausreichenden Kenntnisse über den Gegenstand des Interviews besitzt (Kaiser 2014: 40). 


\subsection{Auswertung der Experteninterviews}

\subsubsection{Die Interviewsituationen und die Fälle}

Unter 4.2 wurde im Rahmen der Fallakquise angedeutet, dass die Interviewpersonen sehr unterschiedliche sozio-demographische Profile aufwiesen und in der Organisationshierarchie zum Teil an sehr unterschiedlichen Positionen der Stiftung standen. So unterschiedlich die Positionen der Interviewten waren, so unterschiedlich waren auch die Gesprächsverläufe und die Interviewerwahrnehmung. Die Reaktionen auf die Interviewanfrage fielen recht unterschiedlich aus und waren gerade zu Beginn der Erhebungsphase mühsam. Über die Gründe kann nur spekuliert werden. Möglich sind unterschiedliche erwerbsbiographische Prägungen, persönliche Umstände oder ein einfacher Zufall. Allerdings wirkte sich die zunehmende Formalisierung der Ansprache mit der Verschwiegenheitserklärung positiv auf die Bereitschaft und die Interviewsituation aus. Unter den Befragten waren vor allem weibliche Mitarbeitende in unteren Hierarchieebenen anzutreffen und gaben entsprechend häufiger an, dass sie das Interview durch diverse, fast immer männliche Vorgesetzte hatten ,absegnen' lassen, abgesehen von einer Ausnahme in einer kleineren Stiftung. Kein männlicher Interviewpartner hat dazu eine Aussage getroffen.

Legt man der Kommunikationsform des Experteninterviews die Kategorisierungen von Bogner/Menz (2009b: 77 ff.) zugrunde, die sie aus der Perspektive der Interviewten gegenüber den Befragenden beschreiben, treffen für die vorliegende Erhebung die Kategorien 1 („Co-Expertin“) bzw. 2 („Expertin einer anderen Wissenskultur”) 3 (,Interviewerin als Laie“), 6 („Interviewerin als Komplizin“) sowie in einem Fall zu Beginn 5 („Interviewerin als Kritikerin“) auf unterschiedliche Interviews zu. So nahmen die jüngeren weiblichen Interviewten, die Interviewten im Doppelinterview der kleinen Stiftung, ein männlicher Interviewpartner ohne erkennbaren Migrationshintergrund mittleren Alters sowie der einzige Interviewte mit Migrationshintergrund (männlich, mittleren Alters) die Interviewerin als „Co-Expertin“ wahr, wobei zum Teil Anhaltspunkte für eine „Komplizenschaft“ (Kategorie 6) abzeichneten. ${ }^{18}$ Die Gespräche setzten ein gewisses $\mathrm{Ma}$ an Wissen über politische Positionen, die aktuelle Gesetzeslage, wissenschaftliche Perspektiven sowie mediale Aufbereitungen der letzten Jahre rund um Themen wie soziale Vielfalt, Gleichberechtigung und gesellschaftliche Teilhabe in Deutschland voraus. Personen und Ak-

18 Die „Komplizenhaftigkeit“ zeigt sich in erster Linie in den Gesprächen nach dem Interview, wenn das Diktiergerät bereits ausgeschaltet ist. 
teure wurden in den horizontalen Gesprächssituationen fraglos als bekannt angeführt, ${ }^{19}$ diskutiert und bewertet. In Fall 2 begegnete die Gesprächspartnerin der Interviewerin misstrauisch (eingangs sehr viele kritische Nachfragen zum Sinn und Hintergrund des Interviews, Kategorie 6: „Interviewerin als potentielle Kritikerin“), schwenkte dann aber um auf Kategorie 1 und 2 der „Co-Expertin“. Die beiden älteren männlichen Interviewten sowie einem weiteren männlichen Interviewten mittleren Alters (Herr U., Herr L. und Herr I.) gingen davon aus, dass es sich bei der Interviewerin um eine Laiin handelte. Nachfragen machten deutlich, dass sie den Wissensbestand der Interviewerin zu verschiedenen gesellschaftspolitischen Themen nicht einschätzen konnten. Zum Beispiel folgte auf die Bejahung der Interviewerin, ja, Herr Ignatz Bubis ${ }^{20}$ sei ihr bekannt, prompt die Frage, ob sie jüdischen Glaubens sei. Erst nach dem aufgezeichneten Interview wurde jeweils gefragt, wofür das Interview überhaupt geführt wurde.

Als Erklärungsansatz kann die Wahrnehmung der Interviewerin (als junge Frau Ende zwanzig mit erkennbarem Migrationshintergrund) angeführt werden. Ähnlich wie Abels/Behrens (1998: 89) ging die Verf. davon aus, dass bei männlichen Interviewpartnern eine „Projektion“ stattfindet, die eine junge Interviewerin als ,harmlos ` erscheinen lässt, vor allem bei älteren männlichen Interviewpartnern ohne erkennbaren Migrationshintergrund, denen gegenüber das sozio-demographische Profil besonders viele Gegensätze enthält. Bei jungen Interviewpartnerinnen konnte eine starke Identifikation beobachtet werden, zumal eine Dame am Ende erzählte (insgesamt nach Bogner/Menz 2005: Kategorie 1 „Co-Expertin“), sie wolle selber promovieren und Detailfragen stellte, als handelte es sich um das Gespräch zweier Freundinnen beim gemeinsamen Kaffeeklatsch. Aus diesem Raster würden im Umkehrschluss die weiblichen Führungskräfte ohne Migrationshintergrund im mittleren Alter herausfallen. Sie fallen insofern auf, als Personen mit diesem Profil besonders misstrauisch auf Interviewanfragen und in der Interviewsituation selbst reagierten.

\subsubsection{Tätigkeitsprofil der interviewten Personen}

Auf Grundlage der Tätigkeitsbeschreibungen zu Beginn der Interviews entstand für alle Interviewpartnerinnen und -partner folgende Visualisierung der hierarchischen Position und Zuständigkeitsbereiche:

19 Zum Beispiel Namen von Bundestagsabgeordneten mit Migrationshintergrund, Details zu Regelungen der doppelten Staatsbürgerschaft, demographische Entwicklungen in Deutschland usw.

20 Ignatz Bubis (1927-1999), Vorsitzender des Zentralrats der Juden in Deutschland 19921999. 
Tabelle 3: Formale Position der Befragten und Zuständigkeiten nach eigenen Angaben

\begin{tabular}{|c|c|c|c|c|c|c|c|c|c|c|c|}
\hline $\begin{array}{l}\text { Position } \& \\
\text { Tätigkeit }\end{array}$ & $\begin{array}{l}\text { Frau X. } \\
\text { Fall } 1\end{array}$ & $\begin{array}{l}\text { Herr D. } \\
\text { Fall } 1\end{array}$ & $\begin{array}{l}\text { Frau I. } \\
\text { Fall } 2\end{array}$ & $\begin{array}{l}\text { Herr N. } \\
\text { Fall } 3\end{array}$ & $\begin{array}{l}\text { Herr U. } \\
\text { Fall } 4\end{array}$ & $\begin{array}{l}\text { Herr T. } \\
\text { Fall } \mathbf{5}\end{array}$ & $\begin{array}{l}\text { Frau T. } \\
\text { Fall } 6\end{array}$ & $\begin{array}{l}\text { Herr L. } \\
\text { Fall } 7\end{array}$ & $\begin{array}{l}\text { Herr I. } \\
\text { Fall } 8\end{array}$ & $\begin{array}{l}\text { Frau K. } \\
\text { Fall } 9\end{array}$ & $\begin{array}{l}\text { Frau } L . \\
\text { Fall } 10\end{array}$ \\
\hline \multicolumn{12}{|l|}{ Vorstandposten } \\
\hline \multicolumn{12}{|l|}{ Geschäftsführung } \\
\hline \multicolumn{12}{|l|}{ Projektleitung } \\
\hline $\begin{array}{l}\text { in operative Arbeit } \\
\text { integriert? }\end{array}$ & ja & ja & limitiert & ja & ja & nein & ja & nein & ja & ja & ja \\
\hline Querschnittsaufgabe?* & nein & nein & nein & ja & nein & nein & nein & nein & nein & nein & nein \\
\hline
\end{tabular}

(Quelle: Eigene Darstellung)

Je nachdem, wie groß die Belegschaft und wie die Organisationsstruktur aufgestellt ist, decken die befragten Führungskräfte einen großen oder kleineren Zuständigkeitsbereich ab. So gab es unter den Befragten solche, denen ,nur ${ }^{6}$ die operative Umsetzung grob vorgegebener Rahmenbedingungen oblag, andere sind von Beginn an sowohl mit der thematischen Ausrichtung als auch mit der konkreten Umsetzung des Vorhabens betraut, wieder andere wirken stark bei der Themensetzung mit und geben die abgesteckten Themen an die Mitarbeitenden weiter. Zwar war die Geschlechterverteilung der Interviewpersonen ausgewogen (sechs männliche, fünf weibliche Befragte), jedoch bewegten sich bereits in dieser kleinen Stichprobe von Führungskräften in Stiftungen die Männer in deutlich gehobenen und mit mehr Verantwortlichkeiten versehenen Positionen. Wie auch Littig (2009) geht die Verf. davon aus, dass „Arbeit, Arbeitsmarkt und Arbeitsstrukturen geschlechtlich geprägt sind“ (Littig 2009: 182) und somit die Stichprobe einfach diesen Umstand widerspiegelt.

Besonders interessant sind dabei die Personen, die in den oberen Hierarchieebenen angesiedelt sind und/oder Querschnittsaufgaben wahrnehmen, bei denen somit davon auszugehen ist, dass sie mehr normative Gestaltungskraft auf die Einrichtung ausüben als Befragte mit rein operativen Aufgaben. Auf die ersteren möchte die Verf. im Folgenden genauer eingehen. Es handelt sich in der Tabelle 3 als Frau X., Herrn N., Herrn T. und Herrn L. bezeichneten Personen. Herr I. war zum Zeitpunkt der Befragung erst seit ca. einem Jahr bei der Stiftung angestellt und soll daher hier ausgenommen werden, obwohl er als Geschäftsführer eine leitende Position innehat. Er war zunächst noch damit betraut worden, bereits initiierte Projekte und Programme umzusetzen.

Durch die eher kleineren Strukturen der ersten Stiftung, ist das Spektrum der Zuständigkeiten der ersten befragten Person, Frau X., sehr groß. Sie sagt, dass sie ,hier die Geschäftsstelle der kommunalen Stiftungen [leitet]. [...] Und 
[ihre] [...] Aufgaben [daraus bestehen] [...] die groben Linien festzulegen [...]“ (I/27-35; [Auslassungen und Ergänzungen durch NDS]) Die Geschäftsführerin agiert auf mehreren Ebenen, wenn sie ihre verschiedenen Aufgaben anführt. So muss sie die inhaltliche Arbeit anschieben und deren Voranschreiten überwachen sowie die erforderlichen finanziellen Mittel im Auge behalten. Bei diesen Vorhaben sind die entsprechenden Mitarbeiterinnen und -arbeiter einzubinden und zu managen, das heißt, die Mitarbeiterinnen und -arbeiter sind in die Entscheidungsfindungsprozesse, zumindest auf inhaltlicher Ebene, eingebunden. Ebenfalls zu ihren Aufgaben gehört die langfristige, thematische Planung von Themenschwerpunkten, wofür ein Gespür für zukunftsweisende Themenwahl nötig ist und die losen Themen zu einem Gesamtkonzept zusammengeführt werden. Ähnlich sieht es mit den Aufgaben eines anderen Geschäftsführers, Herr T., aus.

Als Geschäftsführer liegt meine Aufgabe vor allem darin die auf der einen Seite das strategische Programm der Stiftung zu entwickeln. Und die Umsetzung dieses Programms sozusagen nachzuvollziehen. Aber ich bin nicht in die unmittelbare operative Steuerung der einzelnen Projekte und Programme eingebunden (V/22-25).

Beide Geschäftsführende sind entscheidend an der ,strategischen Ausrichtung“ der Stiftungsarbeit beteiligt. Herr T. überwacht zudem das Gelingen der Vorhaben (,die Umsetzung [...] nachzuvollziehen“). Allerdings wird hier sofort deutlich, dass die beiden Organisationen auf der Ebene der Geschäftsführung auch Unterschiede aufweisen. So muss Frau X. auch die finanzielle Absicherung der Projekte im Auge behalten und gibt die Meilensteine vor (,grobe Linien“), während Herr T. hierarchisch weiter oben angesiedelt und neben der Themeninitiierung wenig bis gar nicht in die operative Arbeit eingebunden ist. Dies mag verständlich sein, sind die beiden Stiftungen von der Besetzung der Hauptamtlichen her unterschiedlich stark. Frau X. leitet eine Geschäftsstelle mit ca. fünf Hauptamtlichen, Herr T. ist Geschäftsführer einer Stiftung mit zwei Sitzen und insgesamt neun Mitarbeitenden.

Weniger als Geschäftsführer, aber auch die Themensetzungen bestimmend, ist Herr L. (Fall VII.) als Vorstandsvorsitzender im Top-Management der Stiftung wiederzufinden.

Ich bin Vorstandsvorsitzender dieser Stiftung. //Ja.//Und meine Aufgaben sind Inhalte, Projekte und Kommunikation. [...] Ich hab einen Vorstandskollegen, //Ja.//der sich um Finanzen, Personal und Organisation kümmert. [...] Aber für den inhaltlichen Bereich bin ich zuständig. Natürlich gilt bei uns das Vier-Augen-Prinzip. Das heißt alle Dinge, die wir machen, machen wir im Einvernehmen. Ja (VII/ 21-35). 
Herr L. kann sich vollends auf die inhaltliche Arbeit, deren Umsetzung sowie um die Kommunikation der Inhalte konzentrieren. Alle infrastrukturellen Fragen behält sein Co-Vorsitzender im Auge. Dennoch gibt es die Möglichkeit, bei schwierigen Entscheidungen den Co-Vorsitzenden als "Sparring-Partner“ („Vier-Augen-Prinzip“) hinzuzuziehen, um eine zweite Meinung zu einem Sachverhalt einzuholen. Damit wird eine perfekte Balance zwischen einer eigenen, hohen Selbstwirksamkeit hergestellt, auf der anderen Seite aber die Verantwortlichkeit bzw. die Konsequenzen eines Vorhabens nicht „auf ein Paar Schultern abgeladen", so die Darstellung.

Neben diesen drei Führungskräften, die allesamt stark in die grundsätzliche Leitung der Organisation eingebunden sind, fällt Herr N. (Fall III.) etwas aus dem Rahmen. Zwar ist er nicht im „Top-Management“ der Stiftung, allerdings erfüllt er andere zentrale Funktionen, nämlich in Form einer Querschnittsaufgabe.

Ich leite zum einen das, das Referat ethnische Divergenz (geändert, Anm. NDS) und soziale Vielfalt (geändert, Anm. NDS) der I-Stiftung. Zudem bin ich zuständig für die Gemeinschaftsaufgabe Transkulturelle Teilhabe (geändert, Anm. NDS). Das ist neben der Frauengleichstellung (geändert, Anm. NDS) die zweite Gemeinschaftsaufgabe der IStiftung. Und hier geht es um die Umsetzung dieser Gemeinschaftsaufgabe auf allen Ebenen (III/15-18).

Herr N. gliedert seine Zuständigkeit in der Stiftung auf drei Ebenen: Er ist Referatsleiter des Ressorts „Ethnizität und soziale Vielfalt“ und leitet hier Projekte. Außerdem widmet er sich der „Gemeinschaftsaufgabe Transkulturelle Teilhabe“, bei dem er „auf allen Ebenen“ der Stiftung als Referatsleiter tätig ist. Zum Schluss erwähnt er seine Aufgabe als Experte in interkulturellen Fragen bei der Organisationsentwicklung hinsichtlich Personalpolitik.

Die aufgeführten Tätigkeitsbereiche zeigen auf, wie unterschiedlich die Tätigkeitszuschnitte der Interviewten waren und wie individuell die Stiftungen letztlich Zuständigkeiten und Aufgabenteilung definieren. Vor dem Hintergrund der Diskussion um die Experten bzw. das Expertenverständnis bei dieser Erhebungsform muss konstatiert werden, dass gerade solche Interviewpartner über besonders viel formelles wie informelles Wissen zugleich verfügten, wenn sie in der Hierarchie der Organisation weiter oben standen oder über Querschnittsaufgaben Einblick in große Teile der Arbeitsabläufe besaßen. Interviewpartnerinnen und -partner, die ,nur' operative Aufgaben wahrnahmen oder noch nicht sehr lange bei der Stiftung arbeiteten, konnten selten Einblick in die normativen Grundsätze der Stiftung geben. 


\subsubsection{Eigendarstellungen im Akteurshandeln}

Im vorliegenden Kapitel 4.3.3 wird analysiert, wie Stiftungen sich selbst als öffentliche organisationale Akteure wahrnehmen bzw. inszenieren. Dabei soll näher betrachtet werden, wie Stiftungen ihre Selbstwirksamkeit einschätzen (Governance) und inwiefern sie Gegenhorizonte zu anderen organisationalen Akteuren bilden. Das Kapitel ist gegliedert in zugespitzte Aussagen, die in Interviews direkt oder indirekt gehäuft gefallen sind. Das Kapitel stellt synoptisch die Codes sowie die Homologien und inhaltlichen Diskrepanzen der jeweiligen Fälle vor.

Transparenz \& Wirksamkeit: „Unsere Stiftung ist objektiv!““

Stiftungen präsentieren sich als relativ unabhängige Akteure, die kaum Rechenschaft über ihre Handlungen ablegen müssen. Umso interessanter war zu beobachten, dass die Interviewten implizit wie explizit erwähnten, dass sie besonders objektiv, weltanschaulich neutral oder transparent handelten. Dabei kommen drei argumentative Ebenen zum Zuge: Die nach objektiven Kriterien generierten Prämissen der Stiftung zu einem gesellschaftlichen Zustand, zum Beispiel über Diskriminierungen von Minderheiten usw., aus der letztlich Projektziele definiert werden, die transparente Umsetzung der Vorhaben selbst und letztlich das empirisch fundierte objektivierte Nachfassen zum Beispiel in Form von Evaluationen oder Wirkungsanalysen.

Konkret thematisierte ein Interviewpartner, dass seine Einrichtung eine Studie durchführen ließ, damit eine bestimmte Annahme der Organisation - in diesem Fall: zu wenig Mitglieder mit Migrationshintergrund in kommunalen Volksvertretungen - verifiziert werden kann und es nicht nur ein „Bauchgefühl“ (III/181) bleibt, sondern es auch „empirisch“ (ebd.) belegt werden kann. Herr N. führt explizit zu einem späteren Zeitpunkt im Interview aus:

Weil wir uns auch jenseits von Ideologie und so weiter einfach fragen, anpacken und versuchen offen zu diskutieren (III/820-821).

Der Experte stellt seine Einrichtung als weltanschaulich neutral dar, obwohl es sich um eine parteinahe Stiftung handelt. Die Formulierung ,wir ... jenseits von Ideologie" soll nahelegen, dass es andere gibt, die ideologisch sind und somit nicht neutral operieren wie sie. Außerdem suggeriert der Interviewpartner mit einem ,und so weiter“, dass es ein Bedeutungsbündel von Vorbehalten hinsichtlich einer Ideologieabhängigkeit gegenüber anderen Akteuren gibt, die der Interviewerin bekannt sein sollen und die er unausgesprochen mit einschließen kann. Dabei agiert die Stiftung auf drei Ebenen: sie fragt, packt 
an und diskutiert offen (vgl. ebd.), das heißt, sie stößt Themen - die rein objektiv auf der Hand zu liegen scheinen - an, führt Änderungsprozesse hinsichtlich dieser gesellschaftlichen Missstände herbei und streut die Erkenntnisse im gesellschaftlichen Umfeld, so zumindest die Eigenwahrnehmung.

Ähnlich äußern sich drei weitere Einrichtungen zum Thema „Transparenz“, „Wirkungsmessung“ bzw. ,weltanschauliche Neutralität". In Fall IV führt der Interviewpartner Herr U. aus, dass alle Programmverantwortlichen die Vorhaben bewerten, sodass es „,dann eben 25-mal begutachtet worden“ ist (IV/603). Im nächsten Satz relativiert der Interviewte diese Aussage aber direkt wieder, indem er sagt: „Man hat zumindest theoretisch die Möglichkeit 25-mal da eine Meinung zu haben. Und die dann versucht zu verdichten und zu einem Urteil zu kommen" (IV/603-606). Er beschreibt also den Idealfall in seiner Organisation. Allerdings scheint man davon nicht immer oder häufig keinen Gebrauch zu machen, dass alle Projekte in einer Art Rotationsprinzip von allen Verantwortlichen begutachtet und bewertet werden. Das suggeriert die Attribuierung als ,theoretisch“. Auffällig ist, dass es hier weiterhin eine interne Evaluation bleibt, wogegen in Fall VII anders vorgegangen wird. Herr L. führt dazu aus,

[...] dass wir in fast allen [Projekten, Anm. NDS] Evaluationen gemacht haben. Wirkungsmessung. Also wir vergleichen die Leistung vorher und nachher. Wir lassen auch mal andere drauf gucken. [...] das ist ja eine Rating-Agentur für gemeinnützige Projekte, [...] So dass wir da mit gutem Recht sagen können: diese Projekte haben ihre Wirksamkeit bewiesen (VII/613-617)

Den Umstand, dass eine externe Rating-Agentur ,draufguckt“ (ebd.), nimmt Herr L. zum Anlass, davon zu sprechen, dass die Projekte in seiner Organisation ihre „Wirksamkeit bewiesen“ (ebd.) haben. Was er genau damit meint, dass „Leistung vorher und nachher“ (ebd.) gemessen wird und wie die Agentur das ganz genau macht, erläutert er nicht. Möglicherweise ist dies auch gar nicht wichtig, weil der Umstand, dass eine (externe) Rating-Agentur eine Analyse durchführt, die Behauptung einer fundierten Wirkungsmessung hinreichend plausibel erscheinen mag. Um dieser Aussage noch mehr Gewicht zu geben, verwendet Herr T. den Ausdruck „,bewiesen“ (ebd.), was Zweifel an der Aussage hinfällig erscheinen lässt. Während also die Stiftung von Herrn T. in erster Linie eine theoretische Möglichkeit einer internen Projektbewertung durch die Programmverantwortlichen selbst anführt, weist Herr L. für seine Organisation auf eine spezialisierte externe Wirkungsmessung hin, die sicherlich auch gewisse finanzielle Mittel verbraucht.

Während die beiden letztgenannten Interviewpartner die objektivierende Projektbewertung in den Vordergrund rücken und der erste Interviewpartner, 
Herr N., Transparenz auf den genannten drei Ebenen angibt, kommentiert Frau T. diesen Aspekt ein wenig anders. Sie sagt:

[...] [wir] haben immer so ein bisschen eher die wissenschaftliche Perspektive und den Draufblick (VI/290).

Die Betonung der „wissenschaftlichen Perspektive“ (ebd.) soll auch hier die Objektivität eines Sachverhalts hinreichend belegen und gibt den Legitimationsrahmen der politischen Handlungen vor. ${ }^{21}$ Neben der Erwähnung einer „wissenschaftlichen Perspektive“ (ebd.), die Objektivität vorgibt, ist der Ausdruck „Draufblick“ eine relativ unübliche Wortform, mit der eine Art „Vogelperspektive" auf Geschehnisse suggeriert. Aus der Vogelperspektive lassen sich Vorgänge am Boden dergestalt beobachten, dass die beteiligten Akteure in ihrem Zusammenwirken durchschaut werden. Somit dient diese Behauptung auch als Rechtfertigung für die Projekttätigkeit und das sonstige Wirken dieser Stiftung, die nämlich diesen „Draufblick“ als Anlass für ihre politischen Interventionen nimmt. Die Eigendarstellung als „objektive“ oder „transparente“ Organisation findet sich in allen Stiftungskategorien.

Reichweite \& Einfluss: „Unsere Stiftung ist (öffentlichkeits)wirksam!“‘

Frau T. führt zum Thema „wissenschaftliche Perspektive“ und „Draufblick“ direkt im Anschluss dazu weiter aus:

Und das Ziel ist letzten Endes auch sowohl in die Öffentlichkeit, aber auch in die Politik hinein Impulse zu geben und Themen zu kommunizieren. //Okay.//Das ist eigentlich von unserer Abteilung das, das Kernziel (VI /291-295).

Sie bindet zwei Richtungen in ihr Wirken ein, der bei sieben der zehn Stiftungen explizit erwähnt worden ist: die Öffentlichkeitswirksamkeit bzw. Reichweite in die breite Bevölkerung oder in bestimmte Zielgruppen hinein auf der einen Seite. Auf der anderen Seite fiel immer wieder der Hinweis, wie auch in diesem Beispiel, dass „Entscheider“, „die Politik“, „Stakeholder“ oder „Eliten“ dahingehend beeinflusst werden sollen, was ,objektiv relevant“" (s. Abschnitt „Unsere Stiftung ist objektiv!“) ist. Frau T. bezeichnet als „Kernziel““ (ebd.) die Aufgabe, ,in die Politik hinein Impulse zu geben“ (ebd.). Das wirkt auf der

21 Dazu Foucault (1978: 51 f.): „In Gesellschaften wie der unsrigen kann die ,politische Ökonomie" der Wahrheit durch fünf historisch bedeutsame Merkmale charakterisiert werden: die Wahrheit ist um die Form des wissenschaftlichen Diskurses und die Institutionen, die ihn produzieren, zentriert; [...].“ 
einen Seite harmlos, würde doch das Synonym „Lobbyarbeit“ sehr viel unpopulärer klingen. Auf der anderen Seite betont sie etwas früher im Interview den Willen der Stiftung, öffentliche Aufmerksamkeit zu bekommen bzw.: „Dass ein bestimmtes Thema in die Öf-, Öffentlichkeit soll“" (VI/242-243). Ähnlich formuliert es eine andere Interviewpartnerin, Frau I.:

Was sind unsere ganz spezifischen Möglichkeiten. Wir können so ein
Thema, wir können den diskursstarke Debatten, können Veranstaltun-
gen machen. Wir können Menschen zusammenbringen. Wir können
durch Bücher vielleicht auch einen Diskurs voranbringen (II/347-350).

Zunächst geht sie davon aus, dass es sich bei den „Möglichkeiten“ der Stiftung um ganz „spezifische“ handelt (weitere Ausführungen s. „Unsere Stiftung ist besonders (innovativ)!"). Das erlaubt den Umkehrschluss, dass aus ihrer Sicht andere (staatliche, zivilgesellschaftliche) Organisationen solche Möglichkeiten nicht - in diesem Umfang - zu haben scheinen. Diese Möglichkeiten bestehen aus „Veranstaltungen machen“ (ebd.) und „Menschen zusammenbringen“ (ebd.) im besonderen und „diskursstarke Debatten“ (ebd.) bzw. „Diskurs voranbringen" (ebd.) im allgemeinen Sinne. Es erfolgt also der Hinweis, dass die Aktivitäten der Stiftung in große, öffentliche Diskurse eingebettet sind, diesen neue Richtungen geben können oder zumindest mit Hilfe der Stiftung erarbeitete Perspektiven Gehör finden, also sich die Stiftung mit der eigenen Position behaupten kann. Im nächsten Beispiel geht der Interviewpartner Herr N. noch einen Schritt weiter und sagt:

Und das (kritische Hinterfragen von gesellschaftlichen Missständen, Anm. NDS) gelingt oft durch skandalisieren. Man muss etwas auch skandalisieren, ja. Nicht irgendwie mit dem erhobenen Zeigefinger, aber die Situation muss man skandalisieren. Die kann man nicht schön reden. Sondern man muss zeigen: schaut hier ist ein erhebliches Defizit, einen riesen Handlungsbedarf (III/391-406).

Der Interviewpartner wirkt sehr viel entschlossener, wenn er immer wieder betont, dass gegenüber einer in einem bestimmten Kontext als Elite aufgestellten Gruppe von Menschen ein gesellschaftlicher Missstand „skandalisiert“ (ebd.) werden müsse. Das erinnert an die Rhetorik gegenüber (massen-) medialer Berichterstattung, die, investigativ betrieben, scheinbar heikle Themen aufdecken oder skandalisieren soll. Es wirkt so, als ob Herr N. davon ausgeht, dass moderate Töne in öffentlichen Debatten nicht beachtet werden und die Konfrontation zu suchen ist, um sich durchsetzen zu können. Hier geht es nicht mehr um „Impulse in die Politik" (VI/292), sondern konkret um die Maßgabe, dass (unbedingt) etwas geändert werden müsse bzw. ein „riesen Handlungsbedarf“ 
(III/406) existiere und die Stiftung klar als politischer Akteur aufzutreten hat. Er formuliert also auch die Handlungskonsequenzen aus der Öffentlichkeitswirksamkeit, die er auch für seine Organisation annimmt. Er führt etwas später weiter aus:

\begin{abstract}
Aber wir müssen immer natürlich auch Modelle entwickeln. Auch, auch Inputs geben für die Debatte. Die, die diskussionsfähig sind. Die natürlich auch kontroverse auslösen können und so. Ja. Also zumindest wir müssen stattdessen immer auch Empfehlungen und Strategien da mit entwickeln und sagen: okay es muss aber eine Politik der Inklusion geben und die könnte so aussehen. So (III/657-661).
\end{abstract}

Für ihn hört die Arbeit der Stiftung nicht mit dem ,(S)kandalisieren“ (III/392) auf, sondern auch das Erarbeiten von Modellen (vgl. ebd.), „Inputs [...] für die Debatte“ (ebd.) sowie, etwas später, „Empfehlungen und Strategien“(vgl. ebd.) gehören mit zur Stiftungsarbeit. Also geht die Stiftungsarbeit über die reine Benennung von Themen - wie intensiv auch immer - hinaus, sondern beansprucht auch eine gewisse „Problemlösungskompetenz“ für sich. Und diese ist Teil der Öffentlichkeitsarbeitsstrategie, gemäß einer Haltung unter der Prämisse: ,wir reden nicht nur drüber, wir machen auch etwas“. Auch hier zeigt sich wieder eine Betonung der Rolle als politischer Akteur, der gesellschaftspolitische Themen nicht nur oberflächlich, sondern nachhaltig angeht. Inwiefern sich hierzu insgesamt die Abgrenzung zu anderen Akteuren verhält, wird unter 5., „Unsere Stiftung ist beliebt!“, behandelt.

Neben dem Aspekt der öffentlichen Wirksamkeit in Richtung wichtiger Akteure in höhere gesellschaftliche Ebenen (Politikerinnen, Politiker, Eliten usw.) zeigt sich eine weitere Selbstdarstellung: Die für die eigene Organisation angenommene gemeisterte Zielgruppenerschließung bzw. Bewerbung der Organisation in der breiten Bevölkerung. Auch hierin sind die Vorstellungen der Expertinnen und Experten als sehr zielstrebig zu attribuieren, zum Beispiel Herr U.:

\begin{abstract}
Aber wir wollen auch neue gewinnen. Immerhin besteht Deutschland aus ungefähr 60, 65 Millionen erwachsenen Bürgern, die wählen können. Und davon, die hatte ich Ihnen ja eben gesagt, sind 45.000, die wir in einem Jahr erreichen nun mal grade nicht viel (IV/696-699).
\end{abstract}

Der Vorsatz, im gesamten Land einen hohen Anteil an (deutschen, volljährigen) Menschen erreichen zu wollen, klingt sehr ambitioniert, obwohl es sich um eine parteinahe Stiftung handelt. Der Interviewpartner geht also davon aus, dass das theoretische Potenzial von „60, 65 Millionen erwachsenen Bürgern“ 
ausgeschöpft werden könnte und keine Grenzen vorhanden sind, die zum Beispiel darin liegen sollten, dass sich Teile der solcherart groß gefassten Zielgruppe aus ideologisch-politischen Gründen in der Stiftung einfach nicht wiederfinden. Auf der anderen Seite schränkt der Interviewpartner sein potenzielles Klientel bezüglich Staatsangehörigkeit und Alter ein: Wichtig ist demnach, dass es Wahlberechtigte sind, die erreicht werden, zumal es sich hier um eine parteinahe Stiftung handelt. Dem Anschein nach soll die Marke der Stiftung auch für die Partei werben, was wiederum dafür spricht, dass auch diese Stiftung als politische Akteurin auftreten möchte. Dies ergibt insofern Sinn, als je nach Fraktionsstärke zum Beispiel im Bundestag die Parteienfinanzierung sichergestellt wird, was schließlich den parteinahen Stiftungen direkt zugutekommt (vgl. Kapitel 3).

Zwar machen die Stiftungen deutlich, dass sie möglichst viele Menschen erreichen möchten, allerdings erklären sie auch, dass sie , an der Spitze der Gesellschaft" mitwirken (möchten). Herr L. führt an dieser Stelle eine Dichotomie an:

So dass wir auch zeigen, wir arbeiten an der Basis und wir arbeiten auch an der Spitze. Wir sind durchaus auch für die Herausbildung einer Elite. Das interessiert uns (VII/309/310).

Neben gemeinwohlorientierten Herangehensweise an „der Basis“ (siehe 3. „Unsere Stiftung ist gemeinwohlorientiert!"), betont der Interviewpartner das Interesse an der „Herausbildung einer Elite“ (ebd.). Neben der „Vergegensetzlichung"von Basis versus Elite wird suggeriert, dass die Stiftung als Organisation die Ausgestaltung einer solchen aktiv anschieben, möglicherweise sogar steuern könne. Somit bringt er seine Organisation als „Elitenschmiede“ ins Spiel. Sieht sich eine Organisation als Elitenschmiede, so geht sie davon aus, selbst Teil derselben zu sein. So wird sehr renommierten Musiklabeln für bestimmte Musikrichtungen, bestimmten Museen für entsprechende Kunstrichtungen oder einigen Hochschulen für bestimmte Fachrichtungen die Möglichkeit attestiert, eine Elite zu beherbergen oder hervorzubringen. Demnach stellen sich die präsentierten Beispielorganisationen als politische Akteure dar, die gesellschaftliche Transformationsprozesse anregen oder einzelne Personen zum Beispiel in Form von Stipendien oder Preisen - zu mehr Bekanntheit oder Befähigung verhelfen, gesellschaftlichen Einfluss zu nehmen, ohne dabei selbst eine ganz zentrale Rolle zu spielen. Dies birgt durchaus Widersprüche. Interessant ist, dass die ,Mitte` gar nicht erwähnt wird. Dass sich Stiftungen häufig unter den Mächtigen wähnen bzw. sich als beliebt empfinden, dabei aber eher im Hintergrund bleiben, zeigt der nächste Fall (II) von Frau I., die auf die Frage zu den Stiftungszielen unter anderem Folgendes sagt: 
Für die (Stakeholder, Anm. NDS) einen Raum zu schaffen um kritisch zu reflektieren, wie offen, wie transparent und wie demokratisch ihre Strukturen sind (II/389-390).

Die Stiftung sieht sich hier als Plattform für andere wichtige Akteure, die aufgrund ihrer Objektivität, Transparenz und Unabhängigkeit (siehe 1. „Unsere Stiftung ist objektiv!") die ideale Basis für Selbstreflektion anderer Institutionen im demokratischen Sinne darstellt. Die Stiftung bildet neben der transparenten Plattform auch letztlich einen geschützten Raum an, da Stakeholder der Verschwiegenheit der Stiftung sicher sein können, postuliert die Eigendarstellung. Damit gewährleistet sie ebenfalls einen bedeutenden Einfluss auf politische Prozesse.

Bevor Herr L. in Fall VII auf die Dichotomie zwischen „Basis“ und „Spitze“ eingeht, nennt er bei den Stiftungszielen den Wunsch, eine „direkte Wirkung für das Gemeinwesen in G-Stadt" (VII/93-94) erzielen zu wollen und führt an, dass die Stiftung ,als private Organisation noch etwas bewirken [kann] immer in Kooperation mit anderen" (VII/92-93). Zum einen schafft er den Gegenhorizont zu öffentlichen Einrichtungen, die scheinbar nicht so durchsetzungsstark agieren können, wie sie als private Organisation. Zum anderen macht er aber deutlich, dass sich Stiftungen vernetzen müssen, damit das Erreichen der Ziele gelingt (siehe: 4. „Unsere Stiftung ist beliebt!“). Es ist schwer nachzuvollziehen, wie viel ,Einfluss' tatsächlich seitens der Stiftungen auf zum Beispiel politische Entscheidungsprozesse ausgeübt wird, da hierfür eine detaillierte Netzwerk- und Prozessanalyse nötig wäre, die letztlich andere Daten erfordert.

Als nächstes soll auf den Aspekt eingegangen werden, dass sich Stiftungen vielfach an das ,Gemeinwohl' oder ,Gemeinwesen' richten, wie es Herr L. auch in diesem Beispiel anführt.

Wohlfahrtsgedanke \& Stiftungszweck: „Unsere Stiftung ist gemeinwohlorientiert!“

Die wenig umfangreiche sozialwissenschaftliche oder politikwissenschaftliche Literatur, die es zum Thema Stiftungen gibt, benennt eine gesellschaftliche Aufgabe ihres Gegenstands als sehr zentral: die „Ergänzungsfunktion“ (vgl. Anheier 2003: 51, siehe Kapitel 3) zu öffentlichen, staatlichen Institutionen, die einen bestimmten Versorgungsauftrag gegenüber der Bevölkerung haben. Durch die Vergabe von Geld- oder Sachmitteln an ärmere Kinder und Jugendliche und deren Familien, Vorlese-Projekte für ausländische Kinder oder die Vernetzung einer bestimmten benachteiligten Interessengruppe gehen Stiftun- 
gen beispielsweise dieser Funktion nach. Im Rahmen der vorliegenden Erhebung benennen es zwei Interviewte sehr deutlich. Herr D. (Fall I) schildert es so:

\begin{abstract}
Also es gibt immer unterschiedliche Zeiten, unterschiedliche Zyklen, wo letztendlich Stiftungen die sozialen Schieflagen auch deutlich beobachtet und dann, ob sie darauf reagieren können. Das ist eigentlich das Schöne an Stiftungsarbeit (I/159-162).
\end{abstract}

Herr D. macht deutlich, dass Stiftungen allgemein ,soziale Schieflagen“(ebd.) genau beobachteten, und zwar zeitlich durchgängig, da es ,unterschiedliche Zyklen“ (ebd.) gibt, die ein Thema gelegentlich relevant erscheinen lassen, das dann nach einer gewissen Zeit wieder obsolet werden kann. ${ }^{22}$ Die Orientierung am Gemeinwohl und die Durchführung von Projekten, die soziale Ungleichheit in der Stadt mindern sollen, bezeichnet er als „,das Schöne an Stiftungsarbeit“ (ebd.). Herr D. macht deutlich, dass für ihn dieses Vorgehen absolut für Stiftungsarbeit steht. Er schließt dabei alle Stiftungen ein und reduziert als Projektleiter die Hauptaufgabe von Stiftungen auf diese Gemeinwohlorientierung. Ähnlich sieht es der Vorstandsvorsitzende einer anderen Stiftung, Herr L.:

(W)ir kümmern uns um das Gemeinwohl. Wir gehen mit frischen, neuen Ideen und mit jungen Leuten an, auch sehr stark in die Stadtteile hinein. Wir reden nicht über die Stadt und sind nicht irgendwo abstrakt in irgendeiner Denkfabrik tätig, sondern wir kümmern uns um die Dinge vor Ort. Wir sind nah dran (VII/275-279).

Er grenzt sich zusätzlich von Organisationen ab, die ,abstrakt in irgendeiner Denkfabrik“ (ebd.) etwas Theoretisches überlegen, statt „nah dran“ (ebd.) zu sein an den sozialen Bedürfnissen der Stadt(teil)bewohnerinnen und -bewohner. Dabei setzt er, anders als Herr D., die Gemeinwohlorientierung nicht absolut für Stiftungsarbeit allgemein, da er die Abgrenzung möglicherweise gegenüber anderen Stiftungen vornimmt, die er dabei im Sinn hat. Er bezieht diesen Aspekt also in erster Linie für die eigene Organisation. Außerdem macht Herr L. deutlich, dass die Stiftung mit ,frischen, neuen Ideen und mit jungen Leuten“ (ebd.) die Projekte angeht. Das zeigt sich wenig später an dem folgenden Zitat:

22 Konkret erwähnt er später im Interview ein Projekt, durch das in den späten 1990er und frühen 2000er Jahren ärmeren Kindern der Zugang zu einem PC erleichtert wurde, dessen Anschaffung damals teuer war. Ein Jahrzehnt später wurde allerdings daraus ein Projekt, bei dem Kindern und Jugendlichen ein maßvoller Umgang mit Technik beigebracht werden sollte. 
Sie müssen auch überhaupt eine Bindung entwickeln können, eine Beziehung entwickeln können. Und das können wir Stiftungen eben besonders gut (VII/450-452).

Herr D. spricht hier von individueller, persönlicher Ansprache potenzieller Dienstleistungsempfängerinnen und -empfänger der Stiftung, die in seinen Augen ,anders“ (VII/447), also nicht über den elektronischen Weg oder über einen Telefonanruf, angesprochen werden müssten, und zwar über den persönlichen Kontakt. Herr D. ist der Ansicht, dass dies „Stiftungen [...] besonders gut“ (VII/452) können, da Stiftungen „Beziehungen entwickeln können“ (VII/451). Er bescheinigt somit Stiftungen die Möglichkeit, den Aufbau eines lokalen Netzwerks voranzutreiben, das letztlich individuelle persönliche Zusammenkünfte möglich macht und sogar die Abgrenzung zur professionellen und privaten Care-Arbeit herausfordert.

Die Gemeinwohlorientierung zeigt sich hier besonders stark bei Stiftungen, die eine starke lokale Einbettung in Struktur (Räume, Personen) und Ausrichtung (Themen, Tätigkeiten) kennzeichnet. Dabei sind insbesondere die beiden dargestellten Fälle zu verorten.

Der in der Literatur als „Ergänzungsfunktion“ (Anheier) reklamierten Bezeichnung für Stiftungen, die zusätzlich zu staatlichen Stellen soziale Dienste anbieten, wird hier also in Teilen entsprochen. Jedoch müsste in einer tiefergehenden Studie erörtert werden, auf welche Stiftungen bzw. Stiftungstypen dies eher zutrifft, weil die parteinahen Stiftungen aufgrund ihrer Außendarstellung vermutlich als politische Akteure ausgenommen werden müssen, da sich in ihren Darstellungen solche Ausführungen nicht finden lassen.

Stiftungen als Pioniere: „Unsere Stiftung ist innovativ!“

Die Interviewten haben sich in vielerlei Hinsicht mit Alleinstellungsmerkmalen positioniert. Ein ganz zentrales Argument ist dabei, dass Stiftungen, anders seien, was bereits in den bisher vorgestellten Zitaten thematisiert wird. Herr L. erwähnt hierzu, dass seine Stiftung „mit frischen, neuen Ideen“ (VII/275-276) an das Gemeinwesen herantrete oder Herr N. führt aus, dass seine Stiftung „Modelle entwickeln“ (III/657) müsse, und nicht nur der Nennung von Problemlagen verhaften bleiben dürfe. Frau I. führt bei ihren Ausführungen an, dass ihre Stiftung durchaus „einen Diskurs voranbringen“ (II/350) könne. Hieraus kann hergeleitet werden, dass sich Stiftungen als Pioniere sehen, wenn es darum geht, wenig behandelte Themen zu bearbeiten oder neue Methoden auszuprobieren, die ein Thema voranbringen sollen. Als Beispiel nennt Frau I.: „Wir können auch Reisekosten bezahlen. Also muss man auch nicht mit einem eigenen deutschen oder deutsch-migrantischen Saft schmoren“ (II/304-305). Hier sollen die Geldmittel die Möglichkeit eröffnen, aus herkömmlichen Strukturen 
auszubrechen und ,über den Tellerrand` zu schauen. Die rein nationale Sicht auf ein neues Vorhaben wertet sie sogar mit dem Ausdruck im ,eigenen [...] Saft schmoren“ gezielt ab. Bereits vorher im Interview führt sie aus:

Also sozusagen die Idee ist: Lernen wie andere eigentlich viel mehr mit dem Blick für die Potentiale älterer Menschen unterwegs sind. Das ist eine sehr stiftungstypische Art sozusagen Best Practice zu transferieren (II/138-141).

In diesem Fall besteht die Pionierarbeit in der Kommunikation von guten Beispielen im Umgang mit älteren Menschen. Sie bezeichnet diese Art des Vorgehens als „eine sehr stiftungstypische Art“ (ebd.). Insofern grenzt sie Stiftungen gezielt gegenüber anderen Akteuren ab, was bisher in den Zitaten häufig deutlich geworden ist und unter 4.3.4 weiter behandelt werden soll. Neben der inhaltlichen Innovationsarbeit, hier am Beispiel der sozialen Differenzgruppe „Ältere“ (weitere Ausführungen siehe 4.3.6), konstatiert Frau I. auch eine methodische Überlegenheit. Sie sagt:

Und wir haben dann wieder gesagt: dann wissen wir aber wie man eine wirklich wirksame Tagung macht. Und haben dann eben nicht hier im L-Forum Veranstaltungen, nicht diese üblichen Tagungen, wo man nur zehn Referate hört und am Ende kaputt ist. Sondern ganz interaktive Formen wie man sich gemeinsam dann die Gelingensmerkmale von so einem Projekt in C-Land oder in P-Stadt in K-Land erarbeitet. [...] Das ist dann wieder sehr stiftungsgemäß (II/381-391).

Die Tagung zu einem Thema soll eine ,wirklich wirksame“ (ebd.) sein, in der nicht „nur Referate“ (ebd.) gehört werden, sondern es sollen ,ganz interaktive Formen“" zum Einsatz kommen, die zu einem Ziel führen, nämlich die „Gelingensmerkmale" (ebd.) eines Vorhabens zu erarbeiten. Auch hier zeigt sich, ähnlich wie in Fall III und VII, der Anspruch, nicht nur zu ,reden“, sondern eine tatsächliche Wirkung erzielen zu wollen, die neuartig ist und gesellschaftliche Gegebenheiten verändert.

Auch in Fall IV zeigt sich, dass gesellschaftliche Themen in Abgrenzung $\mathrm{zu}$ herkömmlichen Institutionen (hier: Schule) angegangen werden müssen. Herr U. führt aus:

Das ist sehr, das ist sehr gemischt. Geht auch manchmal sehr individuellen schulbezogenen Sachverhalten natürlich bis hin zu wirklichen gesellschaftspolitischen Fragestellungen (IV/277-279).

Die Themen in der Schule gelten in gewisser Weise nicht als die wirklich wichtigen Themen. Die „wirklichen“ (ebd.), das heißt wichtigen Themen werden 
außerhalb der Schule behandelt, die dann in der Stiftung aufgegriffen werden. Das Zitat zeigt gleichzeitig ein Überlegenheitsgefühl gegenüber dieser zentralen staatlichen Einrichtung.

Dass Stiftungen Projekte anstoßen, die auf diese Weise „zum ersten Mal“ durchgeführt werden, illustriert Herr L.:

Es ist also ein integriertes B-Stipendium für A-Gruppen. Das erste dieser Art in Deutschland. Wir haben das von Anfang an gemeinsam mit der I.-Landesregierung gemacht als Partner. Und haben auch andere Stiftungen gewonnen hier mit zu fördern (VII/342-345).

Gewöhnlich werden Stipendien an Einzelpersonen vergeben, allerdings nennt Herr L. ein Stipendium, das sich an A-Gruppen richtet und möchte damit die Pionierarbeit seiner Organisation unterstreichen. In Strachwitz (2016: 205) wurde bereits erwähnt, dass Stiftungen häufig auf ihre Innovationsleistung hinweisen, diese aber schwer zu prüfen ist. Auf der anderen Seite klingt es plausibel, dass Stiftungen durchaus innovative Impulse setzen. Da sie nicht gewinnorientiert handeln, können sie risikofreudiger auftreten, weil sie nicht auf das monetäre Gelingen eines Vorhabens angewiesen sind, wie es bei unternehmerischem Handeln der Fall ist. Gegenüber staatlichen Akteuren haben sie den Vorteil des niedrigeren bürokratischen Aufwands und das Privileg, nicht nach allgemeinen Vorgaben handeln zu müssen. Auch dieser Punkt müsste allerdings in weiteren Studien näher betrachtet werden.

[...] sondern sie [bestimmte Zielgruppen, Anm. NDS] müssen anders angesprochen werden als das mit herkömmlichen Mitteln der Fall ist. Also nicht mit Internetseiten, nicht mit Telefonnummern, sondern durch die persönliche Beziehung. Das ist das was wir in unserer Arbeit eigentlich herausgefunden haben (VII/447-449).

Zusätzlich zu diesem Novum weist Herr L. darauf hin, dass diese Innovation in enger Abstimmung mit einer staatlichen Institution erfolgt ist, und zwar mit der zuständigen Landesregierung. So innovativ sich Stiftungen präsentieren, in fast allen Fällen haben die Interviewpartnerinnen und -partner Kooperationen mit ,anderen' erwähnt, ob staatliche, zivilgesellschaftliche oder privatwirtschaftliche Organisationen. Sie treten unabhängig und innovativ auf, können aber letztlich Ziele nur in Kooperationen verwirklichen, weil es beispielsweise ein staatliches Informationsmonopol über Infrastruktur- oder Dienstleistungsbedarfe gibt oder die finanziellen Mittel im Vergleich zu großen Konzernen letztlich zu gering ausfallen. Stiftungen sind insofern interessante Akteure bei der Analyse institutioneller Netzwerkbildung. 
Konkurrenz \& Abgrenzung zu anderen Akteuren: „Unsere Stiftung ist besser!“

Beispielhaft sollen nun drei Zitate angeführt werden, die die Abgrenzung zu anderen Akteuren aus zwei Perspektiven behandeln: die negative Abgrenzung, bei der die Stiftung sich als besonders kompetent wahrnimmt (1), sowie die neutrale Ebene, die im Beispiel als „Allianz“ beschrieben wird bzw. als friedliches Nebeneinander (2).

Herr N. macht in seiner folgenden Aussage deutlich, dass er seiner Organisation mehr Kompetenz zuspricht, als anderen Institutionen.

\begin{abstract}
Also wir wollen dieses Thema (Migration und Geschlechtergleichstellung, Anm. NDS) auch nicht anderen überlassen, die das in vieler zugespitzter und populistischer Art, dass die mal zielen, sondern wir wollen immer auch in diesem Thema eine gewisse Federführung behalten (III/130-133).
\end{abstract}

Neben dem Aspekt, dass er die Aufarbeitung der Themenfelder „Migration und Geschlecht“ „nicht anderen überlassen“ (ebd.) möchte, weil diese es „zugespitzter und populistischer" (ebd.) behandelten, geht er davon aus, dass seine Stiftung bereits aktuell diese Themen „federführend“ (vgl. ebd.) behandelt. Diese Pionierstellung soll sogar „behalten“ (ebd.) werden. Im Umkehrschluss bedeutet das, dass seiner Meinung nach seine Stiftung diese Themen differenziert - nicht zugespitzt - und fachlich - nicht populistisch - behandelt. Er beansprucht zudem, dass dies in der Zukunft im Sinne der Sache auch so bleiben sollte. Hier spiegeln sich auch die Argumente wider, die bereits in den oberen Abschnitten eine Rolle gespielt haben: Die eigene Stiftung wird als öffentlichkeitswirksam, innovativ und selbstlos - im Sinne der sachlichen Aufarbeitung - dargestellt. Diese Argumente finden sich zahlreich auch in anderen Fällen, wenn zum Beispiel die eigenen Vorzüge als „,neutraler Player“ (vgl. II/770) angepriesen werden oder die Durchsetzungsstärke bei Versuchen einen „Diskurs voran zu treiben" (vgl. II/350). Anders als die Gemeinwohlorientierung, bei der klar die parteinahen Stiftungen auszuklammern sind, findet sich das Argument, man sei besonders kompetent in dem, was man tut, in allen Stiftungstypen.

Neben der negativen Abgrenzung führt Herr N. im Fortgang des Interviews besonders explizit aus, was viele Stiftungsvertreterinnen und -vertreter erwähnt haben, wenn es im Rahmen von Projekten und Programmen um Zusammenschlüsse mit anderen Akteurinnen und Akteuren geht.

Und ich glaube aber weiterhin sehr stark an dieses Allianz-Denken. Viele der Aufgaben können wir nur in einer Allianz bewältigen. Nicht 
auf einen Akteur abschieben und sagen „das muss die Politik machen“. Sondern die unterschiedlichen Akteure müssen, müssen einfach zusammensitzen und sich Gedanken machen. So (III/707-711).

Der Begriff der „Allianz“ mutet fast pathetisch an, eher dergestalt, als rücke eine Wertegemeinschaft zusammen und nicht so, als kooperierten Behörden und Verbände in einzelnen gesellschaftspolitischen Projekten. Dem allgemeinen Postulat, die Politik solle Lösungen für einen gesellschaftlichen Missstand entwickeln, widerspricht er. Herr N. ist der Auffassung, dass „unterschiedliche Akteure“ (vgl. ebd.) „einfach zusammensitzen und sich Gedanken machen“ (ebd.) müssen. Diese Perspektive wiederum ist sehr typisch für zivilgesellschaftliche Akteure, da sie sich im Verständnis einer Bürgergesellschaft bei der Lösung gesellschaftlicher Herausforderungen einbringen, statt es staatlichen Autoritäten komplett zu übertragen.

Zum Schluss des Abschnittes, der die Eigendarstellungen der Stiftungen behandelt, soll eine Aussage einer Führungskraft angeführt werden, die mögliche Konfrontationen in der operativen Arbeit konkret anspricht: Sie benennt mögliche Konfliktpotenziale bei öffentlich thematisierten Projektinhalten und zeigt auf, wie die S-Stiftung versucht, diese so gut als möglich zu umgehen. Frau L. sagt:

Es gibt natürlich eine gewisse Konkurrenz auch zwischen Stiftungen: so wer macht die beste Arbeit. Aber das ist keine, also es ist in der Regel keine Ebene, die unsere Arbeit irgendwie stört. Es wird immer dann kritischer, wenn wir auch politisch Position beziehen. Das machen wir als S-Stiftung so gut wie nie. Wir sind bewusst konfessionell neutral und auch politisch neutral (X/320-323).

Demnach gibt sie zu, dass es zwischen den Stiftungen zu Konkurrenzsituationen kommt oder kommen kann. Dies scheint aber die alltägliche Arbeit nicht zu behindern. Positioniert sich aber die Stiftung als politische Akteurin, wird es „kritischer“ (ebd.), wie sie sagt. Die unternehmensnahe Stiftung, die zu den größten deutschen Stiftungen zählt, fährt laut dieser Repräsentantin eine Art „Konfliktvermeidungsstrategie“, indem sie sich als weltanschaulich neutral positioniert.

Bisher lautete die Aussage der Führungskräfte stets, dass es Konflikte kaum gebe oder sogar Stiftungen mit Wohlwollen begegnet werde.

\subsubsection{Stiftungen zwischen Staat und Gesellschaft}

Äußerungen zu Standortbestimmungen im staatlichen und gesellschaftlichen Kontext sind in den Interviews zahlreich vorhanden. An zwei Stellen zeigt sich 
eine sehr vielschichtige Durchdringung mehrerer Aspekte, die die Vernetztheit der Organisation mit öffentlichen Akteuren und die Handlungslogik auf den Punkt bringt. Beispielsweise sagt Herr L.:

Und so wächst hier eben, ist hier eine Stiftung in x Jahren gewachsen aus der Mitte der Stadt heraus. Das ist uns sehr wichtig aus der Mitte der Stadt. Und auch in der Mitte der Stadt gut verankert. In Schulen, in Bildungseinrichtungen (VII/419-422)

In Fall VII stellt der Interviewte die regionale Einbettung der Stiftung heraus. Dabei geht es nicht bloß um die Vernetzung mit anderen lokalen Akteuren, sondern die Narration zur regionalen Einbettung setzt bereits bei der Entstehungsgeschichte an. Die Stiftung sei ,aus der Mitte der Stadt heraus“ (ebd.) gewachsen. Es wird eine Bottom-up-Perspektive angestrengt, die den theoretischen Überlegungen, bei Stiftungen handele es sich um ein „Elitengebaren“ gegenübertritt. Dieser Punkt wird weiter bekräftigt, indem Herr L. weiter ausführt, dass die Stiftung, ,in der Mitte der Stadt gut verankert“ (ebd.) sei. Sodann nennt er zwei Bildungsinstitutionen: Schule und etwas allgemeiner „Bildungseinrichtungen“" (ebd.). Damit wird deutlich, dass die Stiftung sich in erster Linie dem Bildungsbereich verpflichtet fühlt und diesem Bereich die meiste Aufmerksamkeit schenkt.

Im Bereich der Politikgestaltung setzt eine Stiftung andere Akzente. Frau I. führt die Stiftung als Kommunikationsplattform ins Feld, was sich mit entsprechenden Überlegungen zur Stiftungstheorie deckt.

\begin{abstract}
Da laden wir deutsche Entscheider ein, also kommunale Vertreter, Bürgermeister, Leiter von Sozialämtern, Vertreter von Unternehmen, Vertreter von Hochschulen oder ja Behörden. Unterschiedlichste Entscheider, die sozusagen hier einerseits sozusagen mit B-Gruppen arbeiten wollen, andererseits aber nicht so genau wissen was eigentlich, wie man es eigentlich tun könnte. Und denen präsentieren wir ganz konkrete Vorbilder dafür (II/358-363).
\end{abstract}

Die Vernetzung erfolgt laut der Stiftung hier über verschiedene Akteursgruppen und kommunale Strukturen hinweg, auch wenn es einen Schwerpunkt für lokale Akteure zu geben scheint, da sie diese explizit erwähnt werden. Hier sind die Teilnehmenden eher Kundinnen und Kunden und werden von der Stiftung beraten, um Umsetzungsstrategien für unbekannte Themen- und Handlungsfelder vorgestellt zu bekommen, die die Stiftung aber schon im Vorfeld ausgearbeitet zu haben scheint. Es lässt sich durchaus sagen, dass die Stiftung 
sich an der Politik- und weiter, an der Gesellschaftsgestaltung beteiligt, dergestalt, wie es in den Ausführungen zur ,sozialen Wirkung“" (Anheier u. a. 2017a, Striebing 2017) angeführt wird.

Um die jeweiligen Dimensionen genauer zu fokussieren, werden im Folgenden drei Ebenen hervorgehoben, auf die die verschiedenen Aspekte zugespitzt werden können: Nach der Analyse der Aussagen über die regionale Einbettung der eigenen Organisation (1) wird ausgeführt, dass Stiftungen dem Handlungsziel, Menschen - gegenüber dem Staat - zu mehr gesellschaftlicher Teilhabe bzw. zu bürgerschaftlichem Engagement zu verhelfen, sehr viel Bedeutung beimessen (2) wird. Schlussendlich wird sehr genau betrachtet, welche Reziprozitäten in Kooperation oder Konkurrenz zu staatlichen Akteuren sichtbar werden (3). Mit zwei Fallbeispielen zu Gesellschaftsvisionen (4) soll der Abschnitt komplettiert werden.

\section{Regionale Einbettung}

Bis auf die parteinahen Stiftungen, positionieren sich alle großen Stiftungen gegenüber lokalen und kommunalen Institutionen und Akteuren. Erstaunlich ist, dass es sich nur bei einem Fall um eine kommunale Stiftung handelt, letztlich aber dieser Aspekt in allen Stiftungen zur Sprache kommt.

In einem Fall wird insbesondere die gute Vernetzung hervorgehoben, die auch in den bisherigen Zitationen zu diesem Fall mitschwingt. Herr L. bringt es an dieser Stelle explizit zur Sprache:

Da sind wir ja sehr gut verankert in G-Stadt. Wir kennen ja natürlich sehr viele Institutionen, das hilft (VII/475-476).

Das „Vernetzt-Sein“ formuliert er als eine Selbstverständlichkeit („natürlich“), bezieht es allerdings nicht auf Personen, sondern auf Institutionen, die zudem sehr zahlreich sind (,sehr viele"). Im Nachgang wird klar, warum er organisationale Akteure hervorhebt: Bei der Umsetzung gerade sozialer Projekte sind die Stiftungen bekanntlich auf staatliche Hilfe angewiesen, um Bedarfe aufzudecken. Mit dem Zusatz ,das hilft“ macht Herr L. deutlich, dass an dieser Stelle besonders der Fokus darauf gelegt wird, da personenbezogene Vernetzungen nicht in dieser Weise dem Erreichen der Stiftungsziele dienlich sind.

In einem anderen Beispiel spricht Herr D. einen weiteren Punkt an, der die Einbettung in die lokalen Strukturen erörtert. Während Herr L. zuvor die institutionelle Vernetzung benannt hat, geht es im nächsten Beispiel um die Aufdeckung konkreter Förderbedarfe. So sagt Herr D.:

Wir haben die Chance: Wir müssen nicht alles für alle machen, sondern wir können sagen, wir gucken, was hier vor Ort nötig ist (I/188-189). 
Er spielt hier auf den Aspekt des Zwangs zur Machtausübung an, dem staatliche Akteure unterliegen (hierzu siehe 3.: „Reziprozitäten“). Von diesem Zwang kann sich die Stiftung befreien, was Herr D. als „Chance“ (ebd.) angibt. Denn so könne man in Ruhe schauen, welche Bedarfe ganz konkret lokal vorhanden sind. Es geht ihm also sogar um die feinmaschige Versorgung der lokalen Bedarfe gegenüber allen anderen, um die sich der Staat in sehr allgemeinen Strukturen zu kümmern hat. Bei der Frage der Vernetzung mit organisationalen lokalen Akteuren geht es im nächsten Fall nicht um eine positiv angesehene Vernetzung, sondern darum, dass Angebote und Dienste in Konkurrenz zueinanderstehen können. Herr D. führt etwas später im Interview aus:

\begin{abstract}
Aber ich sag mal, örtliche soziale Aktivitäten und vor allem Vereine müssen sie auch bereit sein so eine Stiftungsarbeit zuzulassen. Natürlich sind sie in dem Moment - da hat O (Vorname von Frau X., Anm. NDS) durchaus Recht auch Konkurrenten von freien Trägern. Kann passieren, ne (I/312-316).
\end{abstract}

Neben den bisherigen Ausführungen, Stiftungen seien bei der Umsetzung der Projekte auf die Informationen öffentlicher Akteure angewiesen, gesellen sich nun dieses Mal weitere zivilgesellschaftliche Organisationen hinzu: „Vereine“ (ebd.) und „freie Träger“ (ebd.) werden explizit als potenzielle Konkurrenz hervorgehoben, bei denen es auf der operativen Ebene zu Überschneidungen mit den Handlungsfeldern der Stiftung kommen kann. Inwiefern diese Organisationstypen in der Praxis , aneinandergeraten', ist nicht systematisch aufgearbeitet. Es muss vermutet werden, dass die Konkurrenzsituation regional und handlungsfeldspezifisch für jede Stiftung sehr unterschiedlich ausfällt.

In zwei Beispielen gehen die Interviewpersonen auf die „Stadt“ als lokales Kollektiv ein. Im ersteren Fall führt Frau I. aus:

Und die Stadt ist aber, jeder ist natürlich auch interessiert da dran, dass es der L-Stiftung gut geht, weil sie auch vieles wiederum für die Stadt tut (II/766-767).

Frau I. greift die durchweg positiven Aspekte der Kooperation mit der „Stadt““ auf, die auf Gegenseitigkeit beruhe bzw. bei der die positiven Auswirkungen der Stiftungsarbeit für die lokale Bevölkerung und die lokalen Akteure hervorgehoben werden. Somit unterstützen sich in diesem Fall beide Seiten gleichzeitig, um einen Mehrwert zu erreichen.

Während sich in Fall II eine positive Wechselbeziehung mit der Stadt zeigt, präsentiert Herr L. die Kommune, in der der Stiftungssitz angesiedelt ist, als einen Ort, der durch die Stiftung mitgestaltet werden soll. 
Und wir wollen das, diese Stadt sich eben auch weiterentwickelt zu einem Modell, zu einem, zu einem Vorbild für eine Bürgerstadt, für eine deutsche und zugleich international geprägte Bürgerstadt, die einen inneren Zusammenhalt hat, die aber auch offen ist nach außen. Beides hängt übrigens innerhalb ganz, in einem ganz qualitativen Maße miteinander zusammen (VII/101-106).

Herr L. unternimmt eine normative Zielformulierung, in der die Stadt ein Vorbild („Modell“, „Vorbild“) werden soll für andere Städte. Sie soll nach innen geschlossen auftreten, allerdings für Prägungen von außen offen sein. Aus seiner Sicht hängen diese beiden Umstände miteinander zusammen. Hier zeigt sich eine Funktion von Stiftungen, die in den bisherigen Ausführungen zu einer Stiftungstheorie als „Tradition“ (Strachwitz 2016: 205, siehe oben Kapitel 2) bezeichnet werden, da hier Wertevorstellungen verstetigt werden sollen, die für die Stiftungsvertreterinnen und Stiftungsvertreter gesellschaftlich höchst bedeutsam sind. Dabei verwendet der Interviewte den Ausdruck „Bürgerstadt“ (ebd.), was auf ein aufklärerisches Menschenbild hindeutet. Dieses normative Bild verwendet Herr L. in weiteren Ausführungen, die in den jeweiligen Kapitel-Abschnitten gesondert behandelt werden.

Der Aspekt der regionalen Einbettung von Stiftungen, ihr Mehrwert oder ihr Einfluss ist in der Literatur bisher nicht analysiert und bewertet worden. ${ }^{23}$

Handlungsziel: Bürgerbeteiligung und Befähigung zur gesellschaftspolitischen Teilhabe

Stiftungen legen sehr viel Wert darauf, dass Menschen mündige Bürgerinnen und Bürger sind, die selbst die Initiative ergreifen, um für ihre Interessen einzustehen oder zumindest den Initiativen organisationaler Akteure offen gegenüber treten. Wie im obigen Beispiel soll dieses Handlungsziel möglichst für einen ganzen Standort erreicht werden. Sie propagieren dabei eine starke Bottom-up-Perspektive, da gesellschaftliche Mitwirkung für einen möglichst breiten Teil der Bevölkerung gelten soll. Da Stiftungsgründungen auf individuelle, meistens zivile Initiativen zurückzuführen sind, wird die Stärkung von Bürgerengagement und -kompetenz durchweg hochgehalten. $\mathrm{Zu}$ diesem Punkt äuBern sich die Interviewten in mehreren Fällen, in diesem Fall eher in parteinahen Stiftungen.

Als zentrales Handlungsziel formuliert Herr T. dieses Anliegen wie folgt:

Und das, und das //Ja.//Thema hier ist -wie alles bei der Stiftung- es geht darum sich einzumischen, einzubringen. Ja. Demokratie lebt nicht

23 Mit Ausnahme einer Handreichung zum Gründen von Bürgerstiftungen der ,Stiftung Mitarbeit“: ,Stiften findet Stadt““(1999) von Elke Bauer/André Christian Wolf. 
vom Zuschauen. Demokratie lebt vom Mitmachen, ist einer unserer Leitsprüche (IV/155-157)

Letztlich seien alle Handlungen der Stiftung darauf angelegt, politische Teilhabe breiter Bevölkerungsteile zu ermöglichen. Er begründet es damit, dass die vorliegende Gesellschaftsform, „Demokratie“, nicht durch „Zuschauen“ fortbestehen könne, sondern erst durch die Beteiligung vieler Menschen beständig bleibe. Er erhebt diese Einstellung zum Stiftungsparadigma („Leitspruch“). Etwas später führt er weiter aus:

Das es eben nicht darum geht gewaltmäßige Auseinandersetzungsformen zu finden, sondern eben hier zivilisiert, zivilisierte Formen und auch demokratische Formen, das heißt letzten Endes löst man Probleme in einem Diskurs. In den man sich einbringt, in den man Ideen einbringt, in den man seine Meinung natürlich einbringt, seine Positionen einbringt. Aber nicht mit, nicht mit Gewalt (IV/247-251)

Für den Interviewpartner birgt die Nicht-Teilhabe von Menschen am gesellschaftlichen Gestaltungsprozess somit ein hohes Konfliktpotenzial. Er geht davon aus, dass wenn Menschen, die eine Möglichkeit zur Teilhabe nicht haben oder nicht dazu befähigt werden, zwangsläufig eine gewalttätige Auseinandersetzung mit Mitmenschen oder Repräsentierenden des Staats suchen. Wünsche und Ängste müssten kanalisiert werden, Probleme seien daher in einem „Diskurs"" (ebd.) zu lösen, also vernunftorientiert und im Dialog. Somit ist der Wunsch nach gesellschaftlicher Teilhabe auch eine Rechtfertigung dafür, dass es in der Gesellschaft friedlich zugeht. Herr T. bezieht dieses Paradigma auf ein konkretes Handlungsfeld, indem er sagt:

Wie eben Bürger -weil wir haben natürlich eine Knappheit an Kinderbetreuungsplätzen- wie Bürger dann auch in Eigenregie, in Eigenleistung selber auch dann einen, eine Kita zum Beispiel gründen können. Was ja, was, was möglich ist (IV/584-587).

Die Stiftung ist interessiert daran, den Handlungsspielraum für Bürgerinnen und Bürger zu erweitern, indem sie durch privat ergänzte Strukturen Abhilfe für allgemeine Probleme anschiebt, die in diesem Fall in der ganzen Bundesrepublik vielfältig in der Öffentlichkeit diskutiert werden.

Von der Idee der gesellschaftlichen Teilhabe als Gewaltprävention, ist der nächste Interviewpartner aus einer anderen parteinahen Stiftung weit entfernt. In Zusammenhang mit dem Nutzen des Wahlrechts sagt er:

Es bedarf einer politischen Anstrengung (dass Bürger zahlreich ihr Wahlrecht nutzen, Anm. NDS). Es bedarf einfach auch einer Werbung 
für Demokratie. Und die Anstrengung ist natürlich für beide Seiten. Es ist nicht so eine Einbahnstraße. Alle Bürger und Parteien und so weiter müssen sich anstrengen. Vor allem aber auch Institutionen müssen sich öffnen. Müssen auch signalisieren, dass Menschen willkommen sind mitzugestalten (III/173-179).

Demokratie erfordert eine Kollaboration aus Bürgerinnen und Bürger und dem Staat bzw. politischen Parteien, die hier als Gegensätze präsentiert werden. Da aus der Forschung bekannt ist, ${ }^{24}$ dass namentlich Menschen, die armutsgefährdet sind, seltener an Wahlen teilnehmen, spielt Herr N. darauf an, dass an dieser Stelle auch Handlungsbedarf seitens der Parteien und staatlicher Akteure bestehe. Er sieht allerdings die Hauptverantwortung bei den "Institutionen“ (ebd.), also bei den organisationalen Akteuren, sich zu öffnen und Menschen „willkommen zu heißen“ (ebd.). Die Initiative müssen also zum Beispiel Parteien und Organisationen ergreifen, um Individuen an sich zu binden. Eine weitere Perspektive auf den Punkt der Bürgerbeteiligung eröffnet Frau I.

Nicht im Sinne von Bürger haben die Pflicht irgendwie was beizutragen, sondern es ist auch eine Chancenwahrnehmung (II/94-96).

Sie schwächt den Topos der Bürgerbeteiligung leicht ab, indem sie politische und gesellschaftliche Teilhabe seitens der Bürgerinnen und Bürger nicht zur Pflicht deklariert, sondern davon ausgeht, dass es sich um eine Chance handelt. Es wirkt eher wie ein Luxusgut, eine Option im Leben, von der man profitieren kann, wenn man mag, da man privilegiert ist, in einem Umfeld zu leben, in dem es überhaupt möglich ist. Von einem Pflichtprogramm möchte Frau I. allerdings nichts wissen. In eine ähnliche Richtung geht Herr I. bei diesem Thema, wenn er davon spricht:

Das heißt also [Bürgerkompetenz, Anm. NDS] die Fähigkeit selbst sich als Persönlichkeit zu entwickeln für sein eigenes For-, Fortkommen. Aber eben auch für das Gemeinwesen aktiv zu sein (VII/73-75).

In Fall VII geht es beim Thema Stärkung von Bürgerkompetenz neben dem individualistisch anmutenden Paradigma der Selbstverwirklichung auch um die Nützlichkeit für die Gemeinschaft. Hier werden kommunitaristische Werte angesprochen und es wird ein aufklärerisches Menschenbild deutlich.

Ein Zitat, dass das emanzipatorische Potenzial des Stiftungswesens andeutet, liefert Frau X. mit dieser Ausführung:

24 Siehe Studien zur Analyse von sogenannten „Nichtwählern“, zum Beispiel Armin Schäfer (2015): Der Verlust politischer Gleichheit. Warum die sinkende Wahlbeteiligung der Demokratie schadet. Frankfurt/Main. 
Wenn wir individuelle Hilfe zur gesellschaftlichen Teilhabe gut anderthalb Jahre vor Frau von der Leyen ${ }^{25}$ implementiert haben, ist es nicht so, dass wir dadurch überflüssig geworden sind bei dem N-Projekt, aber dass wir jetzt natürlich neu definieren und sagen: So, das macht jetzt der Staat, ne. Da brauchen wir uns schonmal keinen Kopf drum zu machen. Können also dann darüber hinaus über das, was noch nötig ist, dann unsere Stiftungsmittel einsetzen (I/171-177).

Die Interviewpartnerin macht deutlich, dass ihre Stiftung eine Möglichkeit zur gesellschaftlichen Teilhabe für Kinder aus armutsgefährdeten Haushalten angeht, bevor dies staatliche Akteure umsetzten. Wenn nun öffentliche Stellen diese „Handlungslücke“ schlössen, gibt sie an, dass sie dies zum Anlass nimmt, nach anderen Bedarfen Ausschau zu halten und dass diese Vorgehensweise sogar erwünscht ist. Auch hier spielt die regionale Einbettung durch die Bevorzugung lokaler Bedarfe, die für Stiftungen angedachte Innovationsfunktion, die Fürsorgeleistungen gegenüber der Allgemeinheit sowie die Ergänzungsfunktion mit hinein. Mit Blick auf die weiteren Analysen zum reziproken Verhältnis zum Staat ist festzuhalten: Der Staat ist in den Ausführungen bisher als Kunde bzw. Klient aufgetreten, als gewinnbringender Partner, als Geldgeber oder als ,ordnungsschaffender Überbau', der darauf angewiesen ist, gestaltet und verstetigt zu werden. Diese Ansätze gilt es nun weiter zu vertiefen.

Reziprozitäten mit staatlichen Akteuren und Institutionen

In den Ausführungen von Strachwitz (2016: 204 ff.) zu einer möglichen Stiftungstheorie wurde eine Funktion von Stiftungen erwähnt, die mit den vorliegenden Daten gut abgeglichen werden kann. Der Ansatz „Komplementarität“ beinhaltet die Unterstützungsleistung von Stiftungen gegenüber dem Staat bzw. staatlichen Institutionen. Dahingehende Kooperationen bilden laut den Ausführungen der Befragten eher die Norm; mehr noch, sie machen deutlich, dass sowohl die Stiftung als auch die staatliche Institution gerne kooperieren. Dazu führt beispielsweise Herr L. aus:

Aber in der ersten Ansprache, die wird dann eben über Kinderkrankenschwester oder es geht über unsere Grundschulen, beim Sprachlernurlaub (Name geändert, Anm. NDS) oder beim E-Stipendium. Die empfehlen uns die Familien (VII/472-475).

$\mathrm{Da}$, wie weiter oben ausgeführt, staatliche Institutionen das Monopol über sensible Informationen und einen Großteil der Infrastruktur inne haben, erfolgt die Ansprache für soziale Projekte auch über staatliche Stellen. Und zwar nicht 
über die „lange Hand“ als Vermittler, sondern die Stiftung ist gut eingebettet (,gut verankert” (ebd.)) und pflegt nachhaltige Kontakte zu staatlichen Institutionen. Mehr noch, in diesem Beispiel dürfen die Grundschulen sogar als Nadelöhr zwischen Leistungsempfangenden und Stiftung fungieren.

Das ist einfach auch, sagen wir auch spannend für städtische Einrichtungen, uns zu haben als einen neutralen Player, der keine Interessen hat. Da kann man vertrauen. Wir können wunderbare Moderatoren sein (II/769-773).

Dass die beiden Organisationstypen in der Außendarstellung gerne kooperieren, belegt das Zitat von Frau I. Die Stadt ist Partnerin und Kundin zugleich. Es besteht eine Koexistenz mit unterschiedlichen Rollen, wobei die Stadt eher „Kundin“ der Stiftung ist als umgekehrt und somit die Stiftung in dieser Konstellation eher die Oberhand hat gegenüber den öffentlichen kommunalen Einrichtung. Somit reproduziert die staatliche Einrichtung nicht die Operationsweise der Stiftung, die sie letztlich zu dem macht, was sie ist, sondern die Stiftung tritt als Unterstützerin der Kommune auf. Die Interviewte begründet dies damit, dass die Stiftung sich für die Belange der Stadt engagiert. Zugleich suggeriert sie die Notwendigkeit, dass die Stadt einen „neutralen Player“ (ebd.) braucht, dem man „vertrauen“ (ebd.) kann und der als Moderator (vgl. ebd.) auftritt. Die Aussage, die Stiftung sei eine Organisation, die „keine Interesse“ (ebd.) habe, muss vor dem Hintergrund der in der Literatur besprochen abweichenden Handlungslogik von Stiftungen gegenüber den Sphären ,Staat' und ,Markt ${ }^{`}$ gesehen werden, wenn zum Beispiel Unternehmen gewinnorientiert handeln und entsprechende Marktinteressen verfolgen oder der Staat sein Machtmonopol verteidigt. Stiftungen tun keines hiervon, weshalb sie sich als Akteurin ohne Interessen darstellen können.

Eine besonders vielschichtige Kollaboration wird in Fall V aus den Ausführungen von Frau T. deutlich:

Und so ist $B$-Projekt entstanden. Daraus ist dann ein sehr viel komplexeres und anspruchsvolleres Programm geworden, das eben wirklich den Grundgedanken, der sozusagen auch der Peer-Education, auch da wieder sozusagen im Grunde innerhalb von Schule, nicht schulischen Lernfaktoren zu integrieren, verstärkt. Und, und das wiederum in einer -eigentlich in einer sehr starken- Austauschbeziehung mit, mit dem Staat. Also, denn das B-Projekt wirbt sowohl von unserer, wir finanzieren die Grundinfrastruktur, aber die Lehrertrainings, die sozusagen der Kern sind -wir machen ja Lehrerweiterbildungen im Grunde, im Kern von $B$ - um die Lehrer wiederum zu ermächtigen die Schüler zu ermächtigen. Das ist sozusagen der, der Hintergedanke. Diese Lehrertrainings 
werden jeweils von den Bundesländern dann auch finanziert (V/318327).

Sie führt die Entstehungsgeschichte eines Projekts aus, in dem eine Art Grundfinanzierung durch die Stiftung selbst erfolgt, bei der es Projekttage in Schülergruppen gibt und das „Herzstücks“ des Projekts, nämlich eine Lehrerweiterbildung zur Stärkung interkultureller Kompetenz durch die Bundesländer ergänzt worden sind. Leider ist aus den Ausführungen nicht ersichtlich, wie es dazu gekommen ist. Möglicherweise liegt es daran, dass die Interviewpartnerin noch nicht lange an der Stiftung arbeitet und dies schlichtweg nicht weiß. Frau T. gibt selbst an, dass es eine sehr starke „Austauschbeziehung mit [...] dem Staat" (ebd.) gibt. Ziel ist es, Kompetenzen zu verstetigen, weshalb sich hier das von Strachwitz (2016: 204 ff.) angeführte Indiz zur „Nachhaltigkeit“" von Stiftungen widerspiegelt, die für die Existenzberechtigung von Stiftungen im Rahmen des diskutierten Demokratiedefizits spricht. In einem anderen Zitat im selben Fall zeigt sich eine Art staatlicher Auftrag an Stiftungen.

Ich weiß, dass es vom Innenministerium glaub ich gefördert wird. Und ich, deshalb meinte ich auch, dass die anderen Stiftungen wahrscheinlich ähnliche Projekte haben. Weil das war glaub ich nicht, es war glaub ich für alle Stiftungen so ein ministeriales Projekt. Eigentlich um die Anzahl der Stipendiaten zu erhöhen (VI/217-221).

Auch hier wird ein Projekt angeführt, das von den Stiftungen verwaltet wird, allerdings von ministerialer Seite aus befördert wurde. Hintergrund ist die Idee, das sozio-demografische Profil von Stipendiatinnen und Stipendiaten zu heterogenisieren, also vor allem junge Studierende aus nicht-akademischen, aus migrantischen Elternhäusern und Studierende an Fachhochschulen besonders zu fördern. Wie dies im Detail , angeregt' wurde - als Empfehlung, Weisung, Bitte oder Wunsch - und wie genau die Geldmittel zusammengesetzt sind Aufstockung des Stipendiaten-Budgets allgemein, konkrete Förderung bestimmter Stipendiatinnen/Stipendiaten - wird auch hier leider nicht deutlich. Interessant ist, dass staatliche Akteure sich an dieser Stelle der zivilgesellschaftlichen Ressourcen der Infrastruktur und Handlungskompetenz der Stiftungen bedienen, die sie auch selbst auf- oder ausbauen könnten.

Einen wichtigen Aspekt, der bisher implizit angesprochen wurde und deutlich macht, welche Vorteile zivilgesellschaftliche Akteure gegenüber staatlichen haben, illustriert Herr D.: 
Aber die Problemlagen sind ja auch im politischen Raum länger da, aber um auch wirklich eine Gesetzgebung wirklich für alle unter gleichen Gesichtspunkten und Gleichheitsgesichtspunkten und wie auch immer durch zu setzen, braucht es einfach Vorlaufzeit (I/188-191).

Der Interviewpartner führt aus, dass die Politik zwar Probleme - schnell - erkenne. Jedoch beschränke der politische und bürokratische Aufwand ihr Vermögen ein, zügige und diversen Ansprüchen Genüge leistende Lösungen hervorzubringen. Stiftungen als zivilgesellschaftliche Akteure hätten hier eher freie Hand, da auf sie viele Regularien nicht zutreffen, wie es oben mit den Worten von Frau X. beschrieben worden ist: „Wir müssen nicht alles für alle machen" (I/188-189). Stiftungen heben sich dadurch als privilegierte Akteure hervor, was Herr T. in seinen Äußerungen unterstreicht:

\begin{abstract}
Das heißt also wer noch nicht 16 ist kann eben nicht in den Genuss unserer Angebote kommen. Wir wollen auch nicht, verstehen uns auch nicht so, um bestimmte Defizite im Rahmen der politischen Bildung die, die Schule zu vertreten hat, mit unserem, mit unserer Arbeit ausgleichen. Schon in der Schule, sondern danach ja wenn die dann aus der Schule sind, dann sind sie sozusagen, dann gehören sie zu unserer Kundschaft, das ist klar (IV/160-164).
\end{abstract}

Die Angebote, die seine Stiftung macht, sind seiner Ansicht nach erstrebenswert. Er sieht seine Organisation nicht als „Lückenbüßer“ für staatliche Verfehlungen - zum Beispiel in der schulischen Bildung -, sondern die Teilnehmenden an den Stiftungsangeboten gehörten erst zur „Kundschaft“ (ebd.), wenn sie die staatliche Ausbildung hinter sich gelassen haben.

Die Interviewdaten zeigen ein vielschichtiges, ja schillerndes Verhältnis zwischen Stiftungen und Staat: Mal ist der Staat Geldgeber oder sogar Auftraggeber. Ein anderes Mal Kunde, mitunter fungiert er als (Geschäfts-) Partner. Die Stiftungen sehen sich sowohl in der Ergänzungsrolle zu staatlichen Angeboten, die nicht ausreichend vorhanden sind. In der Literatur bisher nicht verhandelt ist allerdings der Aspekt, dass Stiftungen (lokale) Versorgungslücken zunächst identifizieren können, die später von staatlicher Seite geschlossen werden - und nicht umgekehrt Stiftungen nur Lücken füllen, die der Staat hinterlässt, wenn er sich aus Fürsorgeleistungen zurückzieht. Die Frage nach der Legitimierung kann insofern etwas differenziert werden, als dass davon auszugehen ist, dass bei der Bewertung des Demokratiedefizits nicht die Stiftungen als Organisationen allein zu begutachten, sondern das einzelne Aktionen, Projekte, Kooperationen oder personelle Verwobenheiten in den Blick zu nehmen sind. Stiftungen gerieren sich in der Selbstdarstellung nämlich durchaus als 
selbstständige Akteure, die ihrem Selbstauftrag als zivilgesellschaftliche Instanzen, Bürgerkompetenz zu stärken, flächendeckend nachkommen.

In den bisher herangezogenen Ausführungen präsentieren sich Stiftungen selbst bzw. vermittels ihrer Vertreterinnen und Vertreter als soziale und politische Organisationen, die auf individueller Ebene und auf der lokalen Organisationsebene durch Angebote unterstützen und Menschen zu bestimmten Themen befähigen möchten. Neben dem Wirken in die Mikro- und Meso-Ebene hinein erwähnen allerdings gerade Repräsentanten aus höheren Hierarchieebenen allgemeine Grundsätze und Leitbilder, die die Stiftung befolge. Besonders auf dieser Diskussionsebene ist es nicht immer einfach, die persönliche Weltanschauung der Interviewten von formalen Vorgaben der Stiftung als Organisation zu unterscheiden. Dennoch ist es sehr hilfreich zu verstehen, welche Vorstellungen von Gesellschaftsmodellen in Stiftungen gerade in höheren Hierarchieebenen ,schlummern'. Im Folgenden werden zwei Zitationen dazu angeführt, wie die interviewten Repräsentanten eine heterogene Gesellschaft beschreiben.

In Fall VII, der bereits ausführlich behandelt wurde, ist der Interviewverlauf induktiv: Von den anderen Fällen abweichend wurde hier das Interview situationsbedingt von einer prägenden Begegnung, die der Interviewte eingangs angibt und die nicht lange zurückliegt, hingeführt zu allgemeineren Ausgangsüberlegungen der Stiftungsprojekte und der Stiftung selbst. Dennoch baut der interviewte hochrangige Stiftungsvertreter hier eine Art ,gesellschafts-philosophische“ Narration ein. Er sagt:

\begin{abstract}
Also so unterschiedlich wir sind. Und das ist, für mich ist der Unter-, die Unterschiedlichkeit kein Wert an sich. Sondern eine Vielfalt ohne Einheit ist problematisch. Deshalb ist diese Vielfaltsdiskussion für mich häufig zu verkürzt, weil immer nur die Vielfalt als Selbstzweck sozusagen, Vielfalt gegen Einfalt da gesetzt wird. Das ist ein billiges Wort, sondern die Vielfalt muss organisiert sein. Eine Vielfalt muss auch auf einer, kann nur auf einer gemeinsamen Wertgrundlage sich positiv entwickeln, sonst kommt es zu großen Konflikten. Und sonst kommt es auch zu etlichen Konflikten, die wir in unserer Gesellschaft haben. Nicht, die ja nicht nur von der Gesellschaft selbst produziert, sondern in die Gesellschaft auch hineingetragen werden von außen (VII/222-230).
\end{abstract}

Zunächst geht der Sprecher von einem „wir“ aus, das „unterschiedlich“ sei. Der Begriff ,unterschiedlich“" suggeriert, dass es um individuelle Unterschiede gehe und nicht um sozio-strukturelle Schieflagen zwischen Menschengruppen. 
Jedoch sei diese „Unterschiedlichkeit kein Wert an sich“ (ebd.). Er gibt an, dass einige Menschen einfach nur ein ,Unterschiedlichsein' als Wert kommunizierten und dass diese sogar komplementär zu einer „Einfalt" gehandelt wird. Wen oder was er damit konkret meint, also eine Weltanschauung oder Personen/Parteien lässt sich nicht explizit benennen. Hinzu tritt die ambivalente Semantik der Einfalt im Sinne intellektueller Beschränktheit. Im nächsten Satz fällt der gesellschaftspolitisch häufiger im Zusammenhang mit religiösen oder migrationsbedingten Minderheiten genannte Begriff der „Vielfalt“ und löst „Unterschiedlichkeit“ im Fortgang ab. Dem Topos der „Vielfalt“ möchte der Interviewte den der „Einheit" hinzufügt wissen, was nicht einfach eine physische Außengrenze meint. Das „wir“ wird abgeschirmt durch den Gedanken, dass das „wir“ Grundsätze, ähnliche Leitlinien oder ein allgemein akzeptiertes Regelwerk hat, das sich vom ,Nicht-Wir' unterscheidet. Somit muss für ihn die Gesellschaft (auch) eine Wertegemeinschaft bilden. Das bedeutet im Gegenzug, dass „Vielfalt“ für ihn eine Deskription eines Zustands nebeneinander existierender Individuen ist und kein Miteinander bedeuten muss. Diese Vielfalt muss nämlich erst im Sinne eines Diversity-Managements ,organisiert ${ }^{\text {" }}$ werden. Aus dem Zitat geht nicht hervor, wer dies tun sollte. Vor dem Hintergrund der bisherigen Ausführungen ist davon auszugehen, dass Stiftungen bestrebt sind, bürgerliche Eigeninitiative zu stärken und daher von keinem etatistischen Konzept auszugehen ist. Wenn dies nicht geschehe, komme es zu „großen Konflikten“ (ebd.). Es sind zudem nicht (nur) autopoietisch entstandene Konflikte einschlägig, sondern solche, die ,[von außen] in die Gesellschaft hineingetragen werden [...]“ (ebd.), wahrscheinlich durch die ,Neu-Mitglieder' selbst. Er empfiehlt also ein Narrativ, Regelwerk oder Werte, die von allen im ,Wir' geteilt werden und an denen sich ,Neu-Mitglieder ${ }^{6}$ orientieren mögen. Dabei kann das ,Wir' durchaus heterogen sein, in welcher Hinsicht auch immer. Ohne ein gemeinsames Narrativ bleibt die durchaus erwünschte Vielfalt allerdings als für äußere Einflüsse anfälliges soziales Gebilde zurück.

Somit sind Stiftungen deutlich weltanschaulich geprägt und transportieren Gesellschaftsvisionen. Durch ihre Verortung als Aktionsfeld zwischen „Markt und Staat" betrifft dies in erster Linie Aspekte zum sozialen Zusammenhalt und zur Rolle von Bürgerinnen und Bürgern bei der Interessenartikulation gegenüber staatlichen Institutionen. Sie senden also bewusst Impulse aus, die eine Art normativen Unterbau der Region oder Gesellschaft bewirken sollen.

Auch im nächsten Fall, diesmal zum Schluss des Interviews in Fall III geäußert, benennt der Interviewte die Notwendigkeit eines gesellschaftlichen Narrativs.

Es gibt eine Reihe, die nennen sich: ,, vom Tellerwäscher... “ [Name geändert]. //Interessant.//Genau. Also wir gucken da american dream, american dream und dann an-, analog dazu auch was ist das deutsche. Was 
dürfen sich die Menschen versprechen wenn sie auch die Leistung erbringen. Das ist ein großes Thema. Also //Ja.//was ist die gesellschaftliche Erzählung. Das, was verspricht die Gesellschaft den Menschen. Das sie sagt: wenn Du das und das und das erfüllst bekommst Du das. Gibt es diese Erzählung. //Ah okay//Das, das ist eine, eine grundlegende Frage. [...] Menschen, die sich einbringen, Menschen, die sich engagieren, Menschen, die sich selbst öffnen und auch etwas bewegen. Was verspricht ihnen diese Gesellschaft (III/616-627).

Das Vorbild für eine Veranstaltungsreihe liefert ein internationaler Vergleich: der american dream ist eine idealtypische Vorstellung davon, was man Ankömmlingen in den USA versprechen kann, nämlich Wohlstand bei vorangegangenem Fleiß und Disziplin auf dem Arbeitsmarkt. Man habe sich also die Frage gestellt: Was verspricht Deutschland Menschen, wenn diese ihre ,Bringschuld“ erfüllen, also „Leistung erbringen“ (ebd.), sich „engagieren“ (ebd.), sich öffnen oder etwas bewegt haben (vgl. ebd.)? Herr N. spielt auf die öffentliche Integrationsdebatte an. Es geht also in erster Linie nicht um frisch nach Deutschland zugezogene Personen, sondern um Etablierte bzw. ökonomisch unabhängige Personen.

Während im ersten Beispiel der Interviewte eine typische Innenperspektive einnimmt, sich als ,Ziel' und ,Wir' formuliert, spricht der zweite Interviewpartner aus einer ,Außenperspektive'. Er formuliert eine Notwendigkeit, die er für die Gesellschaft beobachtet, formuliert Bedingungen, die hierfür erfüllt sein müssen, um schlussendlich seine Forderung für etablierte Neuankömmlinge zu unterstreichen. Während ersteres Beispiel das altbekannte Integrationsnarrativ mit einem Leitwertepostulat versieht, beschreibt zweiteres die postmigrantische Perspektive mit dem Fokus auf das post-integrative Versprechen, dass die Postmigration als Theorie in der gesellschaftlichen Praxis nicht eingelöst sieht. Letztlich steuern beide Perspektiven ein ähnliches Ziel an, nur aus zwei Richtungen: Während die eine allerdings auf die Einhaltung dessen pocht, was aus Sicht der anderen noch gar nicht kreiert und implementiert wurde: Ein gesellschaftliches Narrativ, von dem die einen glauben, es existiere bereits, während die anderen glauben, dass es noch fehle.

Der Aspekt des gesellschaftlichen Zusammenhalts und der Gedanke, dass dieser gestärkt, hergestellt oder verteidigt werden müsse, kommt letztlich in fast jedem Interview vor. Während in Fall VII beispielhaft die „Einheit zur Vielfalt" diskutiert und in Fall III ein Zukunftsversprechen für Neuankömmlinge propagiert wird, verbalisieren andere Interviewpartnerinnen und -partner diesen Aspekt als „gemeinsames Verständnis für unsere Demokratie“ (Fall IX/67) oder mit den Worten, dass sie zur gesellschaftlichen „Stabilität beitragen" (II/100) möchten. Dabei ist die Herangehensweise stets die, dass Men- 
schen befähigt werden sollen, sich entweder aktiv ins Gemeinwesen einzubringen oder zumindest sich selbst versorgen zu können, so auch, dass sie mündig sind oder zumindest mündig gemacht werden können. Gerade in Fall VII wird deutlich, dass dies auch auf „bildungsferne Familien“ (vgl. VII/446) explizit angewendet wird.

\subsubsection{Beziehungsherstellung und Selbstverortung}

Um Stiftungen als Akteure im gesellschaftlichen Kontext besser einordnen zu können, werden in diesem Abschnitt drei Fallbeispiele aufgearbeitet, in denen die Beziehungswahrnehmung gegenüber ihren Zielgruppen und ihrem Netzwerk zu Tage tritt. Dies kann nur für die Fälle herausgestellt werden, in denen, erstens, die Interviewten entweder so lange in der Organisation arbeiteten, dass sie dazu nennenswert Auskunft gegeben haben. Oder, zweitens, die Organisation bezog aufgrund ihrer Nähe zu konkreten Personen(kreisen) - zum Beispiel Akteure in Migrantenorganisationen, enge Kooperation mit öffentlichen Einrichtungen - dazu eine Position. Zwei Fälle (II und V) werden in anderen Kontexten ausführlich behandelt. In den folgenden Beispielen ist durch dieses Ausschlusskriterium keine parteinahe Stiftung anzuführen. Neben dem Zufall kann ein Grund hierfür darin liegen, dass sich diese möglicherweise als politische Akteure mit einem stärker auf die Gesamtgesellschaft ausgerichteten Fokus hervortun und sich daher konkrete Beziehungsherstellung zu Zielgruppen seltener finden lässt.

Beispiel Fall I: Vorreiterrolle bei sozialem Engagement durch lokalen Fokus

Fall I wird im weiteren Verlauf im Zusammenhang mit der Nivellierung von Differenzmerkmalen behandelt. An dieser Stelle soll darüber berichtet werden, was die Stiftungsspitze aus Geschäftsführerin und Projektleiter darüber berichtet, wie Zielgruppen wahrgenommen werden bzw. wie sie unter Bezugnahme zu Rahmenbedingungen ihre Projektvorhaben anpassen. Auf die Frage, welches Projektvorhaben aus der Vergangenheit sie ganz besonders hervorheben wollen, antworten der Repräsentant und die Repräsentantin:

Herr D.: Es gab sozusagen einen hohen Zugang an Spätaussiedlern und dann hier in N-Stadt vor vielen Jahren. Wir haben mit dem Projekt meine ich- 1999 oder 1997 begonnen, ne. Da gab's... da gab's ein eklatantes Problem, ein Eingliederungsproblem, ähm, dass man letztendlich mit diesem O-Projekt zunächst über Freizeitgestaltung und Unterstützung bei Freizeitmaßnahmen in Peer-Groups ...ne... versuchte an- 
zugehen. Und dann hat man sehr sehr schnell festgestellt, dass die Freizeitgestaltung nicht unbedingt das Problem ist, da sind die jungen Leute eigentlich selber sehr sehr kreativ...[...] Dann haben wir festgestellt, dass das Problem eher in der Schule bzw. in der individuellen Betreuung der jungen Menschen lag. So hat also dieses Projekt zu einem Zeitpunkt, wo ein Problem also auch latent erkennbar war, hier in N-Stadt, natürlich einen guten Beitrag geleistet. [...] Wir sind jetzt im Moment mit einem anderen Projekt an einer ganz anderen Stelle. Wir wissen, dass die Not bei armen Kindern -ich sag's jetzt mal so- armen Kindern, drückt. Wir haben über 8000 Kinder, die von Sozialhilfe und sonst was abhängig sind. Da drückt der Schuh unheimlich, was Teilhabechancen angeht. Das hat ja auch Frau von der Leyen erkannt. Und wir hier schon früher in N-Stadt, können jetzt an der Schnittstelle arbeiten. [...] (I/140-162).

Herr D. holt historisch etwas aus. Er erläutert, dass es Ende der 1990er Jahre eine Migration von sogenannten Spätaussiedlern nach N-Stadt gab, woraufhin O-Projekt ins Leben gerufen wurde. Das O-Projekt hatte sich zum Ziel gesetzt das „Eingliederungsproblem“ (ebd.) anzugehen, von dem die Stiftung annahm, dass es bestehe. Dabei ging es darum, Freizeitgestaltungsangebote zu initiieren oder zu fördern, damit die jungen Menschen außerhalb von Familie und Schule einen Raum mit Gleichaltrigen haben, um sich zu entfalten und Anschluss zu finden. Wie in Beiträgen weiter oben bereits angeführt wurde, verstehen sich Stiftungen als eigenständige Organisationen, die Projektziele nach eigenen Maßstäben festlegen und revidieren (können). Herr D. führt an, dass genau dies bei O-Projekt geschah: Man habe ,sehr sehr schnell festgestellt, dass die Freizeitgestaltung nicht unbedingt das Problem ist" (ebd.), sodass eine Korrektur erfolgte: Das „Problem [lag] eher in der Schule“ (ebd.), also in einer Sphäre, die staatlich organisiert und obligatorisch für alle jungen Menschen zum Erwerb zukunftswirksamer Kapitalien, wie Zertifikaten und Kompetenzen, verantwortlich ist und bei individuellem Misserfolg einen erschwerten Zugang zum Arbeitsmarkt zur Folge hat. Herr D. erwähnt, dass die Stiftung den Schwerpunkt auf die ,individuelle Betreuung“ (ebd.) setzte und damit scheinbar gut gefahren (,natürlich einen guten Beitrag geleistet“(ebd.)) sei. Die Stiftung korrigiert also das Projektziel der kulturellen Eingliederung hin zur statusrelevanten Unterstützungsleistung auf individueller Ebene und sieht sich dabei auch retrospektiv im Recht.

Herr D. führt nun für die Gegenwart ein Projektbeispiel an, das er wiederum in einem anderen Wirkungsfeld (,an einer ganz anderen Stelle“(ebd.)) verortet. Von der schulischen Jugendhilfe ist die Stiftung nun dazu übergegangen ,die Not bei armen Kindern" (ebd.) in den Blick zu nehmen. Er rechtfertigt sich mit den Worten, dass er das ,jetzt mal so [sagt]" (ebd.), also die Benennung von Kindern, die in Haushalten mit materiell eingeschränkten Möglichkeiten groß 
werden, als ,arm“ zu bezeichnen. Bemerkenswert daran ist, dass es faktisch das korrekte Wort ist, für den Sprecher allerdings eine Abweichung zur öffentlich gängigen Rhetorik darzustellen scheint. Er zählt sodann die Fakten auf: 8.000 Kinder leben in N-Stadt von „Sozialhilfe und sonst was“ (ebd.), haben also Eltern oder Elternteile, die ihren Lebensunterhalt von staatlichen Transferzahlungen bestreiten, die bekanntermaßen lediglich ein Existenzminimum abdecken können. Daher „drückt der Schuh unheimlich, was Teilhabechancen“ (ebd.) angehe, dass nämlich finanzielle Belastungen, die diesen Kindern die Teilnahme an außerschulischen Aktivitäten ermöglichen würden, nicht privat durch die Eltern getragen werden können. Dies habe auch „Frau von der Leyen erkannt" (ebd.), die zum Zeitpunkt des Interviews die Bundesfamilienministerin war und zum 1. April 2011 das sogenannte „Bildungs- und Teilhabepaket“ für Kinder aus Niedriglohn-Haushalten eingeführt hat. Er sagt, dass sie als Stiftung nun „an der Schnittstelle arbeiten“ (ebd.) könnten. Durch die Nähe zu den Projekten und zur Zielgruppe setzt diese Stiftung sehr konkrete Vorgaben in die Tat um und korrigiert genauso schnell Projektziele, wenn im Lauf der Projektlaufzeit deutlich wird, dass der Bedarf an anderer Stelle liegt als von der Stiftung angenommen. So beschreibt der Projektleiter Herr D., wie historisch bedingt von einem „Integrationsprojekt“ zu einer individuellen Schülerhilfe umgeschwenkt wird und Jahrzehnte später nicht der Fokus auf Kinder aus Migrantenfamilien ins Blickfeld rückt, sondern lediglich der sozioökonomische Status als „Koordinatenachse“ für die Vermittlung von Zuwendung angesetzt wird. Welche Rolle nun das Förderprogramm eines Bundesministeriums dahingehend spielt, wird aus den Ausführungen von Herrn D. nicht deutlich. Bevor die Interviewerin nachhakt, führt die Geschäftsführerin Frau X. das Thema vertiefend weiter.

Was bei beiden eigentlich deutlich wird, bei beiden Beispielen, die $\mathrm{P}$ [Vorname von Herr D., Anm. NDS] jetzt genannt hat, ne, dass bevor das System insgesamt eine Lösung finden kann, können wir Stiftungen schon dabei sein. Das ist Jugendsozialarbeit auch für bestimmte Zielgruppen, //Ja//Jugendberufshilfe in den Schulen deutlich etablierter $/ / \mathrm{Ja} / /$, da sind jetzt Strukturen entstanden, jetzt können wir uns zurückziehen. Ne, also, ne. Jetzt gibt es auch in städtischer Personalhoheit diese Sozialarbeiter, die diese Dinge mit aufnehmen. Sicherlich ist auch der Integrationsprozess für die Zielgruppe, die wir damals wie wir sie weiter fortgeschritten, aber das Selbe ist es auch mit den N-Projekt. Wenn wir individuelle Hilfe zur gesellschaftlichen Teilhabe gut anderthalb Jahre vor Frau von der Leyen implementiert haben, ist es nicht so, dass wir dadurch überflüssig geworden sind bei dem „N-Projekt", aber dass wir jetzt natürlich neu definieren und sagen: So, das macht jetzt der Staat, ne. Da brauchen wir uns schonmal keinen Kopf drum zu machen. Können also dann darüber hinaus über das, was noch nötig ist, 
dann unsere Stiftungsmittel einsetzen. Also früher haben wir, ich sag mal, den Vereinsbeitrag von 60 bis 80 Euro für den Sportverein aus NProjekt finanziert, das macht jetzt akkumuliert mit diesen 10 Euro Frau von der Leyen. Thema für uns gegessen. Aber ein Kind braucht auch mal die Fußballerstausstattung oder den Trikotsatz oder auch mal das was weiß ich- Trainingswochenende oder Trainingscamp, ne. Da sind wir dann da, also um wirklich auch eine gelingende Integration auch zu fördern (I/163-183).

Die Geschäftsführerin, die nicht so stark in die operative Projektarbeit eingebunden ist wie ihr Mitarbeiter Herr D., führt aus der Meta-Perspektive aus, wie und warum die Anpassung der Projektziele vorgenommen wurde. Sie sagt, dass „bevor das System insgesamt eine Lösung finden“ (ebd.) könne, seien Stiftungen in der Lage, allgemein unerwünschte gesellschaftliche Zustände zu lindern. Mit „System“ ist an dieser Stelle die staatliche Administration gemeint. Auf die Entwicklung hin nämlich, dass Anfang der 2000er Jahre die Kommune begann, Jugendhilfe institutionell in Form entsprechend geschulter Sozialarbeiterinnen und -arbeiter zu verankern und auch für verschiedene Zielgruppen umzusetzen, zog sich die Stiftung aus der individuellen Förderung zurück. Hinzu kommt die gesellschaftliche Entwicklung, dass ,der Integrationsprozess für die Zielgruppe, [...] weiter fortgeschritten“ (ebd.) ist, also soziales Engagement nicht mehr in erster Linie kulturelle Eingliederung zum Ziel haben muss. Eine ähnliche Entwicklung beschreibt Frau X. für das N-Projekt, bei dem es um die Förderung von Kindern aus armen Haushalten geht. Die Stiftung finanzierte nämlich anderthalb Jahre, bis zur Einführung des „Bildungs- und Teilhabepakets“, genau diese Leistungen, die nun von staatlicher Seite getragen werden. Frau X. sagt, dass sie sich nun „keinen Kopf drum zu machen“ (ebd.) brauchten, allerdings dadurch nicht ,überflüssig“ (ebd.) werden. Wenn nämlich beispielsweise nun die Vereinsmitgliedschaft eines Kindes vom Staat getragen wird, gibt es andere Ausgaben, die für eine ,gelungene Integration“ (ebd.) dennoch fehlen. Frau X. gibt als konkrete Beispiele das Trikot, das Trainingscamp im Sport. Sie beschreibt, wie bürokratische Hilfe nicht vollumfänglich alles abdecken kann, was nah an der Lebenswelt beobachtet benötigt wird. Ist die kontinuierliche Vereinsmitgliedschaft finanziell abgesichert, kommen Familien punktuell doch an ihre finanziellen Grenzen, wenn im Sommer ein Zeltlager für die Mannschaft geplant ist oder alle drei Jahre die Sammelbestellung für ein neues Trikot ansteht. Die Finanzierung solcher Anliegen bezeichnet sie als ,gelingende Integration“ (ebd.), womit Teilhabe am gesellschaftlichen Leben gemeint ist und nicht kulturelle Eingliederung, wie in der öffentlichen Sprache.

Das Beispiel zeigt, dass Stiftungen mit lokalem Fokus sehr präzise die Lebenssituation von Randgruppen fokussieren möchten. Ähnliche Tendenzen 
werden in Fall V durch die lokale Einbettung deutlich, die im Fortgang behandelt werden. Die Interviewpartner Frau X. und Herr D. geben an, Bedarfe vor staatlicher Regulierbarkeit entdecken und angehen zu können, ohne dass sie mit ihrem sozialen Engagement überflüssig werden würden. In diesem Fall suchen sie sich neue „Baustellen“ im lokalen Kontext, die wiederum sehr differenziert bearbeitet werden können. In diesem Fall kommen klischeehaft vorgetragene Gruppenzugehörigkeiten weniger zur Sprache, sondern die Lebensumstände von Menschen werden nüchtern begutachtet und Bedarfe formuliert.

Wie in den vorangehenden Abschnitten dargestellt, verstehen sich Stiftungen nicht nur als soziale Akteure und möchten einen ,gesellschaftlichen Beitrag" zu unterschiedlichen Themen leisten. Im nächsten Fall steht stärker der Aspekt der „Elitenförderung“ im Vordergrund.

Beispiel Fall IX: Change-Agents durch Förderung von potenzieller Elite als zukünftige Multiplikatoren im internationalen Kontext

In Fall IX formuliert die Interviewpartnerin eine Zielsetzung der Stiftung, die sich zunächst wie ein pathetisch vorgetragener Topos ausnimmt:

Also wenn wir in dem Bereich Völkerverständigung arbeiten wollen wir, dass bei Menschen Vorurteile abgebaut werden. Langfristig, dass Frieden gesichert werden kann (IX/231-232).

Mit der Arbeit im Bereich der „Völkerverständigung” (ebd.) sieht Frau K. das Ziel der langfristigen globalen Friedenssicherung (vgl. ebd.) verknüpft. Als Weg zum Ziel formuliert sie den Abbau von Vorurteilen bei Menschen (vgl. ebd.) insgesamt. Sie hebt hier die Summe der individuellen Handlungsebene auf eine die Gesellschaft umfassende. Sie präzisiert den Gedanken mit den Worten:

Wir haben die [Fellows, Anm. NDS] ausgewählt, weil wir gesagt haben: unsere Hypothese ist im Bereich Völkerverständigung, dass in Europa sehr viele unterschiedliche Kulturen existieren. Das diese Vielfalt von Kulturen auch ein positives Merkmal ist. Und das was Europa besonders hervorhebt im Gegensatz zu USA oder anderen Ländern oder Regionen, wo es, wo, wo es ni-, nicht diese Vielfalt gibt. Das dieser kulturelle Reichtum aber auch in sich eine Herausforderung ist. Weil Kultur ja auch eine Grenze darstellen kann. Und das ein produktives Miteinander von Menschen aus verschiedenen Kulturen voraussetzt, dass es Personen gibt, die Kulturaustausch auch sinnvoll vermitteln können. Das heißt wir brauchen Personen, die im internationalen Kulturaustausch zu Hause sind und die Mechanismen kennen, wie man das 
so organisiert, dass diese internationale Zusammenarbeit positiv verläuft. Und wenn wir diese, also eine solche Personengruppe für das Feld, was als auswärtige Kulturpolitik beschrieben wird, rekrutieren wollen, müssen wir früh anfangen. Wir müssen Leute finden, die noch relativ jung sind. Die man prägen kann. Die relativ langfristig an so einem Programm bereit sind teilzunehmen. Nicht nur mal fünf Tage, sondern eben zwei Jahre. Und die wir in dieser Zeit wirklich intensiv begleiten und fortbilden können. Und so sind wir dann automatisch dazu gekommen, dass wir sagen es können nicht die 50-jährigen Profis sein, die nicht, die gehen nicht nochmal zwei Jahre ins Ausland. Es sind eher jüngere Leute, aber es müssen auch Leute sein, die schon ein gewisses professionelles Niveau mitbringen. //Okay.//Das heißt es sind auch keine Schüler oder keine Abiturienten, sondern es sind, es ist eben genau die Gruppe, derer, die eine Hochschulausbildung abgeschlossen haben. Die auch erste Erfahrungen gesammelt haben. Die noch nicht voll in dem Beruf eingestiegen sind. Die in so einem, einem Alter sind, wo sie einerseits sehr viel Wissen mitbringen und auf der anderen Seite noch sehr auch prägbar sind durch praktische Erfahrungen. //Ah okay.//Und so sind wir dann zu dieser Zielgruppe gekommen. Ja (IX/276-303).

Sie beschreibt den Hergang eines Projekts im Rahmen der „Völkerverständigung" und holt dazu ein wenig aus. Die Ausgangsüberlegung sei gewesen, dass in Europa „sehr viele unterschiedliche Kulturen existieren“( (ebd.). Dies bewertet die Interviewpartnerin positiv und stellt es im internationalen Vergleich als regionales Alleinstellungsmerkmal heraus (,,im Gegensatz zu USA und anderen Ländern und Regionen“, ebd.). Diesen „kulturellen Reichtum“ (ebd.) bezeichnet ihn aber gleichzeitig als „Herausforderung“ (ebd.), da Kultur auch eine „Grenze“ (ebd.) darstellen könne. Damit suggeriert Frau K., dass ein Hindernis zu überwinden sei, wenn kultureller Reichtum ,als produktives Miteinander von Menschen“ (ebd.) erlebt werden soll. Dieser Prozess soll laut der Stiftungsvertreterin nun von Menschen moderiert werden, die den Kulturaustausch „sinnvoll vermitteln“ (ebd.) können. Die Personen bedürften aber einer „Spezialausbildung“, damit sie „die Mechanismen kennen“ (ebd.), um dieser Aufgabe gerecht zu werden und zu einem positiven Ergebnis (vgl. ebd.) zu führen. Diese Personen möchte die Stiftung nun ,rekrutieren“ (ebd.) und ausbilden. Dabei hat sie sehr genaue Vorstellungen davon, welches Profil diese haben sollen: Diese Personen sollen „noch jung“ (ebd.) sein, damit man sie noch ,prägen“ (ebd.) könne. Sie müssten langfristig bereit sein, an dem Programm der Stiftung mitzuwirken und zwar „nicht nur mal fünf Tage, sondern zwei Jahre" (ebd.). In dieser Zeit werden sie intensiv begleitet und fortgebildet (vgl. ebd.). Durch diese Vorüberlegungen sei man zu dem Schluss gekommen, dass man nicht die „50-jährigen Profis” (ebd.) auswählen könne, da in dieser 
Gruppe die Bereitschaft, den Wohnort für zwei Jahre ins Ausland zu verlagern, gering sei. Hier wird deutlich, dass die Schulung der Kulturmoderatorinnen und -moderatoren in erster Linie mit einem Auslandsaufenthalt verbunden wird. Auf der anderen Seite sollen diese Personen ,ein gewisses professionelles Niveau mitbringen“ (ebd.). Es soll also objektivierbare Anhaltspunkte dafür geben, aufgrund derer man sich durch die Investition in diese Personen einen Erfolg versprechen kann. Diese Personen seien daher ,keine Schüler, keine Abiturienten" (ebd.), sondern junge Menschen, die bereits einen Hochschulabschluss erworben haben, aber noch nicht fest im Arbeitsleben angekommen sind. Sie meint damit junge Akademikerinnen und Akademiker im Alter von etwa Mitte/Ende zwanzig. Die Interviewpartnerin beschreibt ein Profil, in dem die Balance zwischen Ausbildung (hoch) und persönlicher Prägsamkeit von ihr als sehr hoch eingeschätzt wird. Interessant ist, dass dafür ausschließlich junge Menschen mit Hochschulabschluss ins Auge gefasst werden und keine anderen Absolventen praxisnaher Abschlüsse.

Hier wird deutlich, dass von Stiftungen Geförderte in vielen Fällen als Instrumente fungieren, mit denen man hofft, einen gesellschafts-politischen Effekt zu bewirken oder sich als Akteur zu etablieren, der gesellschaftspolitisch Einfluss nimmt. Das individuelle Weiterkommen der Geförderten steht dabei nicht im Vordergrund. In den präferierten Personalprofilen, die Frau K. mitbringt, engt sich die potenzielle Gruppe der zu rekrutierenden Fellows sehr ein: Absolvierende von Hochschulen, die noch nicht im Berufsleben etabliert und mit wahrscheinlich weiteren, in der Arbeitswelt verwertbaren Merkmalen versehen sind, worin sie sich von ihren Peers unterscheiden. Insgesamt fällt auf, dass die Gruppe der jungen Erwachsenen eine beliebte Zielgruppe von Stiftungen darstellt, so auch im folgenden Beispiel.

Beispiel Fall VII.: politische Philanthropie zwischen sozialem Engagement und Elitenförderung

Fast alle Stiftungen unterhalten Stipendienprogramme für Schülerinnen und Schüler oder Studierende, wobei der Fokus nicht unbedingt auf der materiellen Unterstützung liegt. Gerade nicht-parteinahe Stiftungen führen Förderprogramme mit bestimmten Schwerpunkten durch: begabte Studierende aus MINT-Fächern sind genauso dabei wie sprachlich begabte Schülerinnen und Schüler, denen Auslandsaufenthalte finanziert werden. In Fall VII führt Herr L. dazu aus:

Aber das Personenförderung, Förderung von Talenten aus allen Bereichen, Talent und Charakter, das ist im Grunde genommen unser Profil (VII/366-367). 
Er spezifiziert die individuelle Förderung von Personen als die Förderung von „Talent und Charakter“ (ebd.) und verbindet dies mit der eigenen Organisation mit den Worten, dass hierin ,im Grunde [...] [ihr] Profil““ (ebd.) liege. Während „Talent" als weltlich verwertbares Potenzial gedeutet werden kann, verweist „Charakter“ auf eine ethisch-moralische Komponente. Die Stiftung möchte beide Elemente im Einklang sehen, wenn sich Personen vorstellen, die von ihr gefördert werden sollen. Bereits an anderen Stellen verweist die Stiftung darauf, dass sie „Elite“ fördere (s. 4.3.3). Damit ist die Prämisse verbunden, der zufolge die nötigen Auswahlentscheidungen und -prozesse entwickelt seien und dazu befähigten, sie zu erfüllen. Herr L. macht deutlich, dass sie in der Lage seien, dies auch disziplinübergreifend zu gewährleisten (,,aus allen Bereichen", ebd.). Der Zweiklang aus weltlichem Potenzial und ethischem Topos wird der Organisation einverleibt, indem er als eigenes „Profil“ (ebd.) formuliert wird. Dabei bleibt es jedoch nicht. Neben dem Fokus auf die erfreulichen und Image-freundlichen Projekte, möchte die Stiftung auch „an der Basis“ (VII/309) arbeiten. Als Beispiel dient ein weiteres Förderprogramm:

\begin{abstract}
Da -wenn ein Kind geboren wird- sind viele Familien in unserer Stadt nicht hinreichend vorbereitet, um dieses Kind ja oder sie haben Unsicherheit in der Eltern- und Erziehungskompetenz. Empfinden Unsicherheit und sind unsicher, weil sie isoliert leben. Weil sie materiell in einer schwierigen Lage sind. Weil sie persönlich durch Trennungen und alle möglichen oder gesundheitlichen Beeinträchtigungen Schwierigkeiten haben. [...] Ja die Zugänge schaffen ist für uns auch ein wichtiges Motiv. Zugänge schaffen für Menschen, von denen wir glauben sie sind nicht per se bildungsfern, sondern sie müssen anders angesprochen werden als das mit herkömmlichen Mitteln der Fall ist (VII/327-447).
\end{abstract}

Herr L. begründet die in erster Linie personelle Unterstützung von Familien in der frühen Phase der Elternschaft mit der „Unsicherheit in der Eltern- und Erziehungskompetenz" (ebd.) der Paare, die ein Kind bekommen haben bzw. damit, dass diese „nicht hinreichend vorbereitet“ (ebd.) seien. Er unterstreicht den Aspekt der Unsicherheit abermals unterstützend dadurch, dass diese Personen auch sozial ,,isoliert“ (ebd.) sind, um sodann auszuführen, was die Gründe hierfür sein können: die Eltern sind ggf. „materiell in einer schwierigen Lage“ (ebd.); , ,persönlich durch Trennungen und alle möglichen“ (ebd.) persönlichen Belastungen gekennzeichnet oder müssen ,gesundheitliche Beeinträchtigungen“ (ebd.) kompensieren. Während die Armut sehr stark und die körperliche Verfassung ebenfalls in Zusammenhang mit ungleichheitsspezifischen Denkansätzen behandelt wird, ist der Aspekt der privaten Belastung durch schicksalhafte Erfahrungen neu in diesem Zusammenhang. Dieser Aspekt wird bei der Ermittlung von Förder- oder Unterstützungsbedarf durch fremde Dritte für 
gewöhnlich gar nicht beachtet, da sie möglicherweise sehr temporär wahrgenommen wird. Alles in allem beschreibt der Vorstandsvorsitzende das Klischee der auf staatliche Transferleistungen angewiesen alleinerziehenden Mutter, ergänzt durch Fälle von in Armut lebenden jungen Elternpaaren mit gegebenenfalls gesundheitlichen Einschränkungen, die sich den Anforderungen einer Elternschaft nicht bewusst sind oder waren. Er tut dies auf eine sehr sachliche Art und Weise, die darauf angelegt ist, keine Wertung hinsichtlich der Eigenverantwortung der Betroffenen selbst zu erklären. Diesen Personenkreis, der in der öffentlichen Wahrnehmung als soziale Randgruppe behandelt wird, gilt es nun zu unterstützen und Zugänge zu finden. Dies sei ,ein wichtiges Motiv“ (ebd.) der Stiftung, denn sie gehe davon aus, dass diese Personen ,nicht per se bildungsfern" (ebd.) seien. Das Menschenbild baut letztlich darauf auf, dass persönliche Missstände nicht selbst produziert sein müssen und somit Betroffene in schwierigen Lebenslagen nicht vollumfänglich für ihre Situation haftbar gemacht werden können. So ist für die Stiftung klar, dass es auch für soziale Randgruppen eine Möglichkeit geben muss, sie für ein gesellschaftliches Miteinander und für eine bildungsaspirative Haltung zu begeistern. Dies, so die Stiftung, ist aber eine „Spezialaufgabe“, denn „mit herkömmlichen Mitteln“ (ebd.) sei kein Erfolg möglich. Damit unterstreicht dieser Repräsentant erneut die häufig angeführte Ansicht von Stiftungsvertretern, dass Stiftungen Pionierarbeit leisten. Das heißt, nicht die gängige, für die Massen gemachte Ansprache ist hier angebracht, sondern eine andere, nämlich eine, die ,persönlich“ (VII/450), also speziell ist. Dieser Hinweis ist recht typisch in Zusammenhang mit Zugangsbarrieren zu sozialen Randgruppen und wird in der sozialen Arbeit auch für Migranten-Communities oder Obdachlose angewendet und als ,niedrigschwellige Ansprache“, weil nicht bürokratisch überladen, bezeichnet. Herr L. begründet die Haltung der Stiftung weiter mit den Worten:

Denn die Bildungsangebote sind da für alle. Aber viele Menschen finden den Zugang nicht. Weil sie diese Zugänge und überhaupt diese Angebote aus ihrer eigenen Herkunft nicht kennen. Und da sehen wir uns als wichtige Begleiter, Lotsen. Eigentlich mehr als Lotsen. Bildungsbegleiter gewissermaßen. Um eben grade in den Zuwanderermilieus diesen, den Weg in diese Stadtgesellschaft und in die Chancen, die diese Stadt, am letzten Ende sehr, sehr bedeutende Stadt ihren Mitgliedern bietet diesen Weg zu mahnen. In dem mir die Stadt erklären, G-Stadt erklären, Deutschland erklären. Das glaub ich sind auch nochmal große, wichtige Aufgaben (VII/654-662).

Herr L. spricht eingangs die „Bildungsangebote“ (ebd.) an, die allgemein vorhanden sind und eigentlich durch einen breiten Teil der Bevölkerung angenom- 
men werden. So sind Angebote der kommunalen Verwaltungen, Veranstaltungs- und Sportprogramme von Verbänden oder Kirchen, Kulturprogramme und ähnliches zumeist über Auslagen an öffentlich hochfrequentierten Orten oder Online frei einsehbar. Herr L. weist darauf hin, dass es Menschen gibt, die „diese Zugänge [...] aus ihrer eigenen Herkunft nicht kennen“ (ebd.) und lässt an dieser Stelle offen, ob es sich um die sozioökonomische, die regionale, die ethnische oder ganz allgemein jedwede Sozialisationsbedingung der Kindheit und Jugend handelt, in der außerschulische und außerfamiliäre Freizeitangebote nicht angenommen worden sind. Die Stiftungsmitarbeitenden fungierten in diesem Zusammenhang als „Begleiter, Lotsen“ (ebd.) für diese Personen, die er nun als „Zuwanderermilieus“ (ebd.) spezifiziert. Auch hier wird ein Klischee angesprochen, das an Ghettos und soziale Brennpunkte in westlichen Großstädten erinnert. Während im obigen Beispiel die Beschreibung der Lebenslage sehr differenziert und sachlich war, klingt der Begriff des „Milieus“ nun verallgemeinernder und weniger fokussiert auf die genauen Umstände, die mit einer solchen Lebenslage verbunden sind. Es wirkt, als ob ihm Einzelfälle in diesem Fall persönlich nicht so präsent seien wie die weiter oben beschriebene Zielgruppe, die aus armen Familien besteht. Vom Begleiter für Bildungsangebote geht Herr L. nun dazu über, der Zielgruppe den „Weg in diese Stadtgesellschaft“ (ebd.) ebnen zu wollen und vergisst nicht zu erwähnen, dass es sich um eine „sehr bedeutende Stadt“ (ebd.) handelt, die für ihre Mitglieder „Chancen“ (ebd.) bereithält. Aus der "Stadt" allgemein wird sodann konkret der Standort (G-Stadt) projektiert. Als nächstes wird daraus induktiv ein „Erklärprogramm“ für ganz Deutschland. Diese Begleiter-Programme für Mitglieder aus „Zuwandermilieus“ (ebd.) sind aus seiner Sicht ,große, wichtige Aufgaben" (ebd.), also eine Herausforderung, der nachgegangen werden muss, möchte man nicht die Zukunft von G-Stadt verspielen.

In diesem Beispiel wird ein Dreiklang deutlich: die Stiftung fokussiert die aus ihrer Sicht als „oberer“ Rand wahrgenommenen Gruppen der Gesellschaft und möchte Elitenförderung betreiben. Sie möchte, als zweites Ziel, soziale Randgruppen, die durch unterschiedliche Misslagen geprägt sind, sozial unterstützen, da niemand zurückgelassen werden brauche, da alle Menschen vernunftbegabt seien und für verschiedene Themen sensibilisiert werden könnten. Als drittes gilt es Menschen aus ,,anderen Kulturen“" an hiesige Strukturen, Traditionen und Regelwerke heranzuführen, da sie sonst entgleiten könnten. Die Stiftung verbindet die Aktivitäten eines Wohlfahrtsverbands mit dem Aufbau und der Pflege von Sozialkapital in die höheren gesellschaftlichen Hierarchieebenen hinein unter Hinzunahme missionarischer Welterklärerschaft: Der Ausdruck politische Philanthropie mag hier durchaus zutreffen. 


\subsubsection{Argumentationskonstellationen hinsichtlich sozialer Differenzkategorien}

Die bisherigen Auswertungen offenbarten ein Selbstverständnis von Stiftungen, das sie als demokratiefördernde, philanthropische, einzigartige sowie gemeinwohlorientierte Organisationen mit hoher Wirksamkeit erscheinen lassen. Zur Analysierbarkeit des Umgangs mit sozialer Differenz wurde vorab in Kapitel 3 herausgearbeitet, dass neben der Verortung im gesellschaftlichen Gefüge und der Beziehungsherstellung zu sozialen Gruppen die Benennungspraxis zu sozialer Differenz eine zentrale Rolle spielt. Diese sollte nach ihren Konnotationen, Wechselbeziehungen zwischen gegebenen Differenzmerkmalen und den genauen Benennungskontexten analysiert werden. Im nächsten $\mathrm{Ab}$ schnitt (4.3.6) werden die Analyseergebnisse zu diesem Vorhaben unter den Aspekten „Reproduktion von (alternativen) Narrationen“ (1), „Aufwertung sozialer Differenz“ (2), „Negierung sozialer Differenz“ (3) sowie „Relativierung" (4) gegliedert.

Reproduktion von (alternativen) Narrationen

In öffentlichen Diskussionen bzw. der öffentlichen Wahrnehmung zirkulieren diverse Narrationen über Personengruppen, ethnische oder andere Minderheiten. Ihre konkrete Entstehungsgeschichte verläuft ähnlich wie im wissenssoziologischen Kontext über Deutungsmuster in Diskursen, ist selten öffentlich bekannt oder kann im Nachhinein nicht immer nachvollzogen werden. Diese Narrationen verweilen persistent im Unterbewusstsein aller möglichen Akteure - so auch in organisationalen Einheiten des Dritten Sektors.

Den ,Klassiker ' der Benennungspraxis bildet die „Reproduktion“ sozialer Differenz bzw. in der öffentlichen Meinung vermuteter Narrative zu sozialen Gruppen. Durch die schlichte Erwähnung der Differenz selbst wird sie herausgehoben, fokussiert und zirkuliert gegebenenfalls unkontrolliert weiter. Die Differenzreproduktion der Organisation wird entsprechend mit der öffentlichen Wahrnehmung begründet.

Als Paradebeispiel dient eine Art Forschungsprojekt mit jungen Menschen eines bestimmten Glaubens. Vorbereitend zu einer Broschüre, die für die öffentliche Verbreitung im Bereich „Radikalisierung-Prävention“ (VI/255) bestimmt sei, führt Frau T., Projektleiterin, aus:

Wir haben wie gesagt eine qualitative Studie gemacht. Wir haben Interviews geführt mit muslimischen Jugendlichen, 40 oder 38 muslimische Jugendliche. Diese Studie haben wir oder die, die Auswertung veröffentlicht, eine Broschüre für Pädagoginnen und Pädagogen und eine Tagung. Eine Fachtagung (VI/247-254). 
Im Gewand einer empirischen Untersuchung werden dutzende „muslimische Jugendliche“" (ebd.) befragt. Die Vorstellung der Interviewergebnisse erfolgt auf einer Fachtagung, in deren Gefolge auf Grundlage der Interviews eine „Broschüre für Pädagoginnen und Pädagogen“ (ebd.) herausgebracht wird. Das konkrete wissenschaftliche Erkenntnisinteresse der Studie tritt aus den Daten - auch auf Nachfrage - nicht hervor, ebenso wenig die Resonanz oder der Verbreitungsgrad der Broschüre. Frau T. erwähnt in diesem Zusammenhang, dass das Arbeitsfeld „Radikalisierung-Prävention“ (VI/255) noch sehr frisch sei. Die Themenwahl ist stark durch die öffentlichen Debatten beeinflusst. Indirekt gibt Frau T. dies zu, indem sie wenig später weiterführt, man wolle auch bestimmte Impulse ,in die Öffentlichkeit, aber auch in die Politik hinein“ (VI/293) geben. Interessant in diesem Zusammenhang ist, dass sich die Stiftung erklärtermaßen nicht als eine Produzentin für ,didaktisches Material oder sowas" (VI/290) versteht. Warum die Stiftung ohne pädagogische Expertise dennoch in diesem Zusammenhang auftritt, wird nicht deutlich. Reproduziert wird die Narration, junge Menschen muslimischen Glaubens seien prophylaktisch vor politischen Radikalisierungen zu schützen und schulische Fachkräfte bräuchten diesbezüglich Hilfestellungen, die bisher fehlten. Schließlich entbirgt sich an dieser Stelle die Vorstellung, diese jungen Menschen hätten das Potenzial, radikalisiert zu werden oder es zu sein. Intersektional betrachtet kreuzen sich an dieser Stelle die Kategorien der religiösen bzw. ethnischen Zugehörigkeit und des Alters bzw. Lebensabschnitts (in schulischer Ausbildung).

Insgesamt schimmert das Merkmal der Religionszugehörigkeit mit dem Verweis auf aktuelle öffentliche Debatten der letzten Jahre deutlich hervor. In Fall X führt L. an:

Wir wollen für mehr Wissen über den Islam sorgen bei den Nicht-Muslimen in Deutschland. Wir wollen das Muslime und Nicht-Muslime sich besser verstehen und besser zusammenleben können und sich, und auch eben da Vorurteile abbauen (X/236-239).

Die Stiftung wolle, dass sich „Muslime und Nicht-Muslime [...] besser verstehen“ (ebd.) und auch „Vorurteile“ (ebd.) abgebaut werden. Dafür soll gesorgt werden, indem man „Wissen über den Islam“ (ebd.) für Nicht-Muslime aufbereitet, was letztlich zum genannten Ziel führen soll. Es wird eine Grenzziehung zwischen „Muslimen“ und ,allen anderen“ geschaffen, die soziale Zuschreibung der Religionszugehörigkeit auf einer gesellschaftlichen Makro-Ebene reproduziert. Die Beschreibung dieses Projektvorhabens transportiert die Botschaft, dass das Zusammenleben von „Muslimen und Nicht-Muslimen“ allgemein nicht gut funktioniert, konfliktbehaftet ist und es hier Maßnahmen bedarf. 
Bedenkt man, dass die Herstellungsabsicht sozialer Differenz in der vorliegenden Studie zweitrangig ist, sondern die Differenzmarkierung grundlegend betrachtet wird, ist im vorliegenden Fall eine Mischung eines Otherings (Castro Varela/Dhawan 2005) und eines Boundary makings auszugehen (Lamont/Molnár 2002) wie es in Kapitel 3 diskutiert wurde. Ohne auf weitere Differenzmerkmale zu rekurrieren, werden zwei Bevölkerungsblöcke konstruiert, die sich im gemeinsamen Alltag scheinbar frontal gegenüberstehen. Allerdings ist in diesem Fall, anders als im klassischen Verständnis von Othering augenfällig, dass die Sprecherin sich selbst keiner Gruppe zuordnet. Die Perspektive verbleibt auf einer übergeordneten, beide Gruppen überblickenden Ebene. Dadurch wird eine symbolische Grenzziehung wie es im Boundary making thematisiert wird, vorgenommen. Da es sich um symbolische und zunächst nicht um soziale, also Ungleichheit markierende Grenzziehungen handelt, vervollständigt das Othering das Boundary making in diesem Fall bei der Beschreibung der Hervorbringungspraxis und der Reproduktion sozialer Differenz in Bezug auf religiöse Gruppen.

Neben der Religion findet die Reproduktion einer Narration in einem weiteren Projekt, das ebenfalls von Frau L. erwähnt wird, zu einem anderen Differenzmerkmal statt. Hier heißt es:

Das ist eine Internetseite für Jugendliche, die Jugendliche, Jugendlichen politische Themen näher bringen soll. Und sie zum politischen Engagement aktivieren soll (X/151-154).

Ziel ist es, dass sich junge Menschen politisch engagieren sollen (vgl. ebd.). Dafür wurde eine „Internetseite“ (ebd.) eingerichtet, über die „Jugendlichen politische Themen näher“ (ebd.) gebracht werden sollen. Die Interviewte geht davon aus, dass sich Jugendliche andernfalls politisch desinteressiert zeigen und hier eine solche Intervention nötig ist. In Anbetracht gängiger Klischees über die Beschaffenheit gegenwärtiger „Jugend“ ist auch diese Narration eine Reproduktion. Der Begriff ,aktivieren“ (ebd.) macht deutlich, dass es sich um eine Art Potenzial handelt, das aus einer passiven Zielgruppe eine aktive machen soll. Insofern werden jungen Menschen an dieser Stelle bestimmte Eigenschaften zugesprochen, die sich negativ-komplementär zu Vorgängergenerationen verhalten.

Jenseits von Religionszugehörigkeit, Generationen und Migration hat die Stiftung in Fall IX einen „Intensivkurs Deutsch als Zweitsprache“ (IX/79) für Stipendiatinnen und Stipendiaten ins Leben gerufen. Frau K., die Projektleiterin, erläutert das Programm folgendermaßen:

Des Weiteren bieten wir ja Unterstützung beim Spracherwerb an. Das heißt einen Intensivkurs Deutsch als Zweitsprache. Der sich, ja Deutsch 
als Zweitsprache, wie das, natürlich der Begriff auch schon sagt, an Stipendiatinnen und Stipendiaten, die zwar schon Deutsch können. Die es als zweite Muttersprache erworben haben. Aber die vielleicht noch gewisse Defizite einfach haben. Die sagen: ich fühl mich nicht wohl, wenn ich vor anderen Studierenden ein Referat halten muss. Oder so (IX/78$84)$.

Die Stiftung bietet „Unterstützung beim Spracherwerb an“ (vgl. ebd.). Diese Maßnahme wird sofort präzisiert: es handelt sich nämlich um einen Sprachkurs für fortgeschrittene Deutsch-Sprechende, aber für muttersprachliche Anfänger. In der Fachsprache wird dafür der Begriff „Zweitsprache“ verwendet, weil es sich um eine Zielgruppe handelt, die zunächst eine Herkunftssprache (zum Beispiel aufgrund eines Migrationshintergrunds) und im Kleinkindalter erst Deutsch als zweite Muttersprache erworben hat. Die Leistungsunterschiede in der Sprachkompetenz fallen dann, wie auch bei Deutsch unter „Erstsprachlerinnen und -sprachlern“, unterschiedlich aus. Der Kurs soll die Sprachkompetenz vor allem für das professionelle Umfeld stärken. Als Beispiel für ein solches mögliches „Defizit“ (ebd.) gibt die Interviewpartnerin an, dass Stipendiaten von sich erzählten, dass sie sich ,nicht wohl“ (ebd.) fühlten, wenn sie „,vor anderen Studierenden ein Referat halten“ (ebd.) müssten. Oder in ähnlichen Kontexten, möchte Frau K. ergänzen. Hier verwischt die Grenze zwischen Spracherwerb, der Kompensation von Defiziten und dem Erlernen von sogenannten Soft Skills, wenn es darum geht, neben Fachwissen weitere persönliche Fähigkeiten, zum Beispiel Präsentationstechniken, auszubilden. Allerdings wird es durch die Gesprächspartnerin intuitiv als sprachliches „Defizit“ (ebd.) formuliert, obwohl man sich vorstellen darf, dass sich diese jungen Menschen vorab in mehreren Durchgängen vor Jurys und Auswahlkommissionen vorgestellt haben, gut präsentieren können und für ein Stipendium für gut genug befunden worden sind. Statt es differenziert zu betrachten, wird aus der Perspektive klassischer Integrationspostulate mit dem Fokus auf Spracherwerb und Sprachkompetenz argumentiert. Es findet ein ,doing ethnicity while undoing class" statt: es geht eigentlich um die Kompensation von Defiziten aufgrund der sozialen Herkunft. Sie beruht auf der allgemeinen Annahme, dass das Erlernen sogenannter Soft Skills wie Präsentationstechniken und professionelles Auftreten mit der sozialen Herkunft zusammenhängt und bei einer privilegierten Herkunft weniger positiv ausgeprägt ist. Allerdings wird dieser Umstand stattdessen mit der sprachlichen Sozialisation bzw. der migrantischen Herkunft der Stipendiaten begründet.

Im nächsten Fall geht es, ähnlich wie im oben angeführten, um ein Stipendienprogramm, das eigens für junge Leute ins Leben gerufen wurde, deren Eltern oder Großeltern nicht in Deutschland geboren oder aufgewachsen sind. Auf 
die Frage, ob man bei der Umsetzung der Projektvorhaben auf „Gegenwind“ gestoßen sei, überrascht Interviewpartner Herr I., Geschäftsführer der Stiftung, mit Ausführungen zur Zielgruppendefinition im Projekt. Kurz zuvor hatte er die schwierige (Arbeitsmarkt-) Situation vieler zugewanderter Eltern der Stipendiatenschaft ausgeführt.

Genauso ist eben auch das Ges-, die Überlegung ist es noch sinnvoll, dass T-Stipendium sich nicht öffnet gegenüber Nicht-Migranten, also, also das ist ja die Frage warum man nur auf Migranten setzen sollte. Ist eine absolut legitime Frage. Die ist auch meiner Meinung nach richtig, dass man auch sagen muss ein Programm, was eigentlich nur Migranten betreut, fördert dadurch die Integration an sich. Aber was wirklich so ist und deshalb haben wir es auch so gesagt, dass, das es schwierig ist einen anderen Definitionsrahmen zu finden (VIII/274-280).

Der Interviewte führt zu Beginn aus, dass es nach einiger Zeit kritische Stimmen gab, warum man das Stipendium nicht für Nicht-Migranten öffnete (vgl. VIII/266-270), sodass die „Überlegung“ (ebd.) im Raum stand, auch NichtMigranten zum Förderprogramm zuzulassen (ebd.). Diese „Überlegung“ wird vom Interviewten als ,absolut legitime Frage“ (ebd.) bezeichnet. Für ihn ist Kritik daran, dass in dem Programm ausschließlich Migranten gefördert werden, absolut legitim. Dennoch begründet er die Entscheidung damit, dass dies - die Förderung von Migranten - die „Integration an sich“ fördere. Demnach ist die Förderung von Nicht-Migranten nicht oder zumindest weniger notwendig und würde auch weniger zur Integration beitragen. Obgleich der Interviewte auch selbst nicht ganz von dieser Annahme überzeugt ist („Aber was wirklich so ist und deshalb ..." (ebd.)), sei letztlich der Grund, warum man nichts an der ursprünglichen Zielgruppendefinition geändert habe, schlicht der, dass „es schwierig ist einen anderen Definitionsrahmen zu finden“ (ebd.). Und hier seien es nun einmal ausnahmsweise Migranten, die gefördert werden. Sonst stünden viele andere Programme allen anderen offen (vgl. VIII/280 282). Mit der Schwierigkeit, ,einen anderen Definitionsrahmen zu finden“ (vgl. ebd.), meint der Interviewte, dass es der Stiftung vor allem darum geht, junge Menschen aus prekären Lebenslagen zu unterstützen. Das oberste Ziel ist also der Abbau von (Chancen-) Ungleichheit, hier durch den Abbau von Armutsfolgen wie geringeren Bildungschancen. Am einfachsten ist dieses Ziel somit zu erreichen, wenn man die Zielgruppe als migrantisch klassifiziert, da man dann die Linderung von Armut am effektivsten erreichen kann. Gleichzeitig will die Stiftung einen Beitrag zur Integration - auch wenn der Begriff hier nicht näher definiert ist - leisten, womit sie etwa die ausschließliche Förderung von Migranten und den Ausschluss von Nicht-Migranten legitimiert. Das impliziert, dass Herr L. - und letztlich die Stiftung - Migration und Armut 
eng aneinander gekoppelt wahrnimmt bzw. ungesagt diese beiden Kriterien erfüllt sein müssen, um ein Stipendium der Stiftung erhalten zu können. Migration und Klasse stehen hier in Wechselbeziehung zueinander. Dass es andere Stiftungsvetretende abweichend sehen, findet sich in den unteren Beispielen „(3) Negierung“ und ,(4) Relativierung“. In diesem speziellen Fall wird allerdings das Merkmal „Migration“ explizit reproduziert, um eine Zielgruppe abzustecken, die, ohne es explizit zu erwähnen, für prekäre Lebensverhältnisse steht. In diesem Beispiel reflektiert der Interviewte diese Zielgruppendefinition auto-organisational, problematisiert diese, aber stellt sie als alternativlos dar. Es findet ein ,doing ethnicity while doing class“ statt, was aus der Perspektive der Intersektionalitätsforschung bedeutet, dass beide Differenzmerkmale hier gleichzeitig wirken, Ungleichheit markieren und in die Argumentation einfließen, ohne weiter ausdifferenziert zu werden. Migration scheint untrennbar mit Armut gekoppelt zu sein.

Die Reproduktion sozialer Differenz findet sich in den Daten aus einer weiteren Perspektive, nämlich dann, wenn sie durch Privilegierung sozial markierter Gruppen vorgenommen wurde. Diese spielt auch für die Kommunikationsarbeit der Stiftungen eine wichtige Rolle, was in einigen Fällen ganz direkt geäußert wird. Herr I. benennt dieses Vorgehen deutlich als solches:

\begin{abstract}
[...] dass man einfach das Gefühl hatte vor 10 Jahren, dass das Potential und auch die Leistung von jungen Migranten im Grunde mehr in den Blickpunkt kommen sollten. Man wollte weg von dieser Negativsichtweise. Und hat dadurch versucht ein Stipendienprogramm aufzubauen für junge Migranten, die engagiert sind und auch talentiert sind, um sie weiter zu fördern (VIII/112-116).
\end{abstract}

Hier wird von einem „Potential“ (ebd.) gesprochen und auch von der „Leistung von jungen Migranten“ (ebd.), die „,mehr in den Blickpunkt“ (ebd.) gerückt werden sollen, weil man sich „,weg von der Negativsichtweise“ (ebd.) bewegen wolle. Aus diesem Grund sei das Stipendiatenprogramm ins Leben gerufen worden, um diese Menschen, „die engagiert sind und auch talentiert sind“ (ebd.) fördern zu können. Die Ausgangsüberlegung geht dahin, dass junge Migranten allgemein als nicht leistungsstark und nicht talentiert gelten, sodass mit einem Stipendienprogramm die Gruppe bzw. Teile davon, als genau das Gegenteil präsentiert werden kann, durch Privilegierung der Gruppe junger Menschen nichtdeutscher Herkunft, die es - unausgesprochen - eigentlich schwer hat im Leben. Intersektional betrachtet, wird Lebensabschnitt bzw. das Alter (jung), der Erwerbstatus (noch in Ausbildung) mit einem nicht deutschsprachigen Elternhaus (ethnische Herkunft) verknüpft und als Zielgruppe festgeschrieben, die es bevorzugt zu behandeln gilt. Implizit gehen die Akteure, die das Konzept entwickelt haben, davon aus, dass es diese jungen Menschen 
ohne die Unterstützung der Stiftung schwer haben, ihren Bildungsweg störungsfrei zu beschreiten. Somit wird die Beschaffenheit des Programms als Bekämpfung sozialer Ungleichheit verstanden, die an dieser Stelle und mit dieser Personengruppen-Konstellation als besonders nötig empfunden und reproduziert wird. Allerdings geht es dabei nicht (nur) um die individuelle Unterstützungsleistung, die die Stiftung erbringt, sondern um das Evozieren eines bestimmten Bildes über eine bestimmte Personengruppe, das nach außen gekehrt werden soll. Die Unterstützung der Stipendiatenschaft dient daher als Mittel zum Zwecke der Kommunikationsarbeit der Stiftung, die gelungene soziale Aufstiege präsentiert.

Ähnlich kommuniziert dies in Fall V Herr T. In seinen Ausführungen überlagern sich ebenfalls diese zwei unterschiedlichen Argumentationsebenen, die zum einen im obigen Beispiel vorkommen. Er nennt diese allerdings expliziter. Er sagt:

\begin{abstract}
Also wir haben eben und, und das sagte ich eben, da gibt es bei uns so eine, eine Entwicklung des Denkens eigentlich weg von der Ethnizität hin zum sozio-ökonomischen Status. //Okay, mhm.// Trotzdem haben wir nach wie vor ein Stipendienprogramm, das sich ausschließlich an, an Studierende mit Migrationshintergrund //Ja.// wendet. Das hat, ich weiß das hat immer die Gefahr dieser ethnic trap, aber gleichzeitig ist, das ist eigentlich, und wir, wir sehen es auch als Kommunikationsinstrument letztlich, weil wir sozusagen in einer Debatte, die von, von Büchern wie, wie den von Sarrazin geprägt werden. Es einfach für wichtig halten sozusagen auch die, die andere erfolgreiche Seite zeigen zu können (V/450-459)
\end{abstract}

Er gibt offen an, dass öffentliche Debatten über ethnische oder sonstige Minderheiten durch Bilder geprägt werden, die für gewöhnlich eine negative Bilanz zu Migration und ethnischen Minderheiten ziehen. Daher sei es „wichtig“ (ebd.) „die andere erfolgreiche Seite zeigen zu können“ (ebd.). Somit fungiert hier eine Fördermaßnahme als Kommunikationsinstrument und weniger als soziales Projekt, mit dem benachteiligten Menschen geholfen werden soll. Der Anstoß dafür kommt von außerhalb der Organisation durch die Wahrnehmung der öffentlichen Diskussion, an der die Stiftung durch das Aufzeigen eines „Gegenentwurfs" partizipieren möchte. Anders als im vorbenannten Fall reflektiert dieser Interviewte das Vorgehen, indem er von einer „ethnic trap“ (V/455) spricht und angibt, man habe trotzdem diese Zielgruppe fokussiert (und damit reproduziert), weil es für die Kommunikationsarbeit der Stiftung wichtig war, ein bestimmtes „Image“ zu transportieren. Hier wird eine alternative Narration angestrebt, die wieder durch die Privilegierung der benachteiligten Gruppe er- 
folgt, um doch unabsichtlich die dazugehörigen Narrative mit zu reproduzieren. Dieses Motiv wird relativ häufig geäußert. In Zusammenhang mit einem Sprachlernprojekt zur Unterstützung von Schulen sagt der Interviewpartner in Fall VII, dass man „Bilder des Gelingens [...] in die Stadtgesellschaft hinein spiegeln“ (VII/561-562), indem man gerade bildungsferne Familien „erreichen, gewinnen und halten“ (VII/562) wolle. Dieses Vorgehen ist kompatibel mit privatwirtschaftlichen Strategien der Imagepflege und wird in diesem Kontext im Diversity-Management vollzogen. Dabei geht es nicht direkt um den wirtschaftlichen "Gewinn“, sondern um das Vorzeigen einer bestimmten „Geisteshaltung“" (Stuber 2002) und einer gesellschaftspolitischen Wertehaltung, der gemäß Minderheiten gegenüber offen begegnet werde. Da Diversity nicht das gleichzeitig angegebene Ziel der Bekämpfung sozialer Ungleichheit erfasst, überlagert sich hier das Paradigma der Intersektionalität mit dem der Diversity.

Der „Reproduktionsklassiker“, der in Rekurs auf die öffentliche Wahrnehmung bestimmter Bilder und Narrative beruht, trat im Rahmen der Interviewdaten immer wieder hervor. Jedes Mal wird eine in der öffentlichen Anschauung zu Personengruppen wahrgenommene Ansicht aktiv angegangen, reproduziert und durch „Gegenmaßnahmen“ förmlich „bekämpft“. Die Idee, dass zu Zielgruppen Argumente ergänzt oder sogar durchkreuzt werden, wird explizit im nächsten Abschnitt ausgearbeitet, in dem es um die „Aufwertung sozialer Differenz" geht.

\section{Aufwertung sozialer Differenz}

Neben der Reproduktion allgemeiner bzw. alternativer Narrative in Hinblick auf soziale Differenz geht es im nächsten Abschnitt darum, Paradigmenwechsel bei der Benennung sozialer Differenz aufzuzeigen, die die Interviewten explizit oder implizit als Absicht angeben. Diese Form des Umgangs mit sozialer Differenz findet in erster Linie durch Aufwertung sozialer Gruppen statt.

Aufwertung sozialer Differenz findet sich zu verschiedenen Differenzkategorien. Mit dem Alter und dem Lebensabschnitt argumentiert auch Frau I. in Fall II, wenn sie das Projektvorhaben ihrer Stiftung begründet:

Und ältere Menschen haben nicht nur bessere Konditionen als je vorher in der Geschichte. Sind gebildeter, sind vermögender, sind gesünder als je Alte vorher. Das ist auch ein Potential. Die können sich einbringen. Die können arbeiten. Die können sich engagieren. Das wollen sie auch. Die wollen die Generationen zusammenhalten (II/338-342) 
Die Prämisse dieser Ausführungen geht dahin, dass ältere Menschen per se körperlich schwächer, kränker und weniger gebildet sind als der Bevölkerungsdurchschnitt bzw. gegenüber den Vorgängergenerationen. Aktuell haben aber laut der Interviewten Ältere nun ,,bessere Konditionen als je vorher in der Geschichte" (ebd.), was auf einen vorläufigen Zenit in diesem Punkt hinweist. Dabei geht es nicht nur um körperliche Fitness, sondern Ältere sind ,gebildeter, sind vermögender, sind gesünder" (ebd.) als ältere Menschen früher. Dieser Umstand wird nun als „Potenzial“ (ebd.) gedeutet. Angepriesen wird, dass sie „sich einbringen, [...] arbeiten [...] engagieren [können]“ (ebd.). Der Aktionsradius älterer Menschen wird durch die Interviewpartnerin als weit eingestuft. Hinzu kommt, dass sie glaubt, dass ältere Menschen dies auch tun möchten (vgl. ebd.), und zwar auch die „Generationen zusammenhalten“ (ebd.). Somit evoziert sie das Bild von aktiven Seniorinnen und Senioren, die körperlich und geistig nicht so eingeschränkt sind, wie es in der öffentlichen Wahrnehmung zumindest früher gewesen ist. Interessant ist der Aspekt des intergenerationalen Zusammenhalts, der von den Älteren gewährleistet werden könne. Somit spricht Frau T. auch der Zielgruppe das Potenzial zu, soziales Kapital herzustellen, aufrecht zu erhalten oder zu bewahren. Die Umsetzung des Vorhabens findet dann im Rahmen einer wiederkehrenden Veranstaltungsreihe statt, in der Praxisbeispiele aus dem Ausland vor Ort vorgestellt und deren Übertragbarkeit in den deutschen Kontext diskutiert werden (vgl. II/350-363). An dieser Stelle findet die Aufwertung nur gegenüber einer Differenzkategorie statt, und zwar gegenüber dem Lebensabschnitt bzw. dem Alter von Menschen. Das Beispiel mit der Aufwertung von sozialen Kategorien kann fortgesetzt werden mit der Ergänzung um eine weitere Differenzkategorie im gleichen Fall. Frau I. sagt hier:

$\mathrm{Na}$ das ist doch toll. Das ist doch großartig, dass wir ältere Migranten haben, weil die haben, die verfügen über mehrere kulturelle Zugehörigkeiten. Die haben Zugang zu einer Community. Die können besonders Kinder unterstützen aus den entsprechenden Zuwanderungs-Communities. Die haben eine ganz andere Autorität. Das ist ganz großartig (II/371-375).

Die intersektionale Verortung von ,älteren Migranten“ hinsichtlich Alter und Migrationsstatus bzw. Ethnizität dient auch hier als Ressource und soll genutzt werden, um aus den jeweiligen Communities „Kinder [zu] unterstützen“. Einer Menschengruppe, die in der öffentlichen Wahrnehmung als unsichtbar bis problematisch gilt, wird hier eine Ressource zugesprochen und somit ein $\mathrm{Pa}$ radigmenwechsel angestrebt. Dabei unterscheiden sich die älteren Migranten von den älteren „deutschen“ Senioren durch ihre ,ganz andere Autorität“ (ebd.), was wiederum auf eine Essentialisierung hindeutet. In diesem Fall kann 
,doing difference“ als Essentialisierung von ,doing age and ethnicity ' in die Theorienlandschaft eingruppiert werden. Letztlich schwingt in der Aussage mit drin, dass diese „Zuwanderungs-Communities“ (ebd.) besonders schwierig im Umgang sind und die älteren Migranten hier behilflich sein könnten. An dieser Stelle wird durch die Aufwertung der intersektional hergestellten Gruppe ,älterer Migranten“ gleichzeitig die Reproduktion bestimmter Narrative der restlichen Migranten vorgenommen, die - ähnlich wie oben gezeigt eine spezielle Unterstützung bräuchten, um ihren Alltag bewältigen zu können.

In Frau I.'s Ausführungen wird soziale Differenz, sofern explizit genannt, auffallend häufig aufwertend reproduziert. Bei der Darstellung von eingeladenen Expertinnen und Experten in Fachgesprächen sagt sie wenig später.

Man würde bei uns immer versuchen im Auftritt, in der Zielgruppe möglichst breit unterwegs zu sein. Und denkt Diversity glaub ich mit. Aber im Sinne der Sache, nicht um politisch korrekt zu sein. Sondern damit der Austausch wirklich gut ist. Das dann wirklich unterschiedliche Traditionen und Denkweisen aufeinander stoßen (II/298-302).

Somit macht erst die Mischung der Gäste die Qualität aus. In diesem Fall wird die Anwendung eines Diversity-Ansatzes nicht als Kommunikationsinstrument nach außen inszeniert, sondern um ,wirklich unterschiedliche Traditionen und Denkweisen“ (ebd.) zusammenzubringen und einen qualitativ hochwertigen Austausch zu erzielen. Damit wird Diversity zu Zwecken der Profitmaximierung genutzt, wenn auch nicht in einem primär monetären Kontext. $\mathrm{Da}$ es sich, wie in Kapitel 2 aufgezeigt, bei Stiftungen um Organisationen des Dritten Sektors handelt, ist der Profit ohnehin in diesem Zusammenhang als im Wesentlichen immateriell zu verstehen.

Die Interviewpartnerin, die weiter oben den „Intensivkurs Deutsch für Zweitsprachler/innen" angepriesen hat, an denen Stipendiatinnen und Stipendiaten mit Migrationshintergrund teilnehmen, führt sehr viel später im Interviewverlauf in Bezug dazu aus:

Und dieses Potential auch irgendwie zu nutzen. Sieht man ganz klar in dem Bereich Sprachförderung. Das wir halt sagen es ist ja nicht nur als Defizit zu sehen wenn jetzt jemand Deutsch als Zweitsprache in einem Intensivkurs in Anspruch nehmen soll, sondern es ist ja auch irgendwo eine Bereicherung. Weil die Person, dadurch, dass sie noch eine zweite Sprache spricht und noch eine dritte Sprache spricht, die wiederum ja auch was weitergeben kann. Und einfach wir wollten diese Kapazitäten, die es irgendwo gibt, auch noch stärker hervorholen und einfach offenlegen und transparent machen für die Stipendiatenschaft (IX/246-252) 
Sie sagt, dass das „Potenzial [von jungen Menschen mit einer anderen Sprache als Deutsch als Erstsprache, Anm. NDS] auch irgendwie zu nutzen“" (ebd.) sei, besonders im Bereich Sprachförderung. Das, was sie weiter oben als sprachliches „Defizit“ (IX/82) angegeben hat, versucht sie nun in ein positives Licht zu rücken. Es sei ,ja auch irgendwo eine Bereicherung [...] [weil] die [Person, Anm. NDS] auch was weitergeben kann“ (IX/246 ff.). Die Formulierung lässt allerdings darauf schließen, dass sie entweder noch nicht immer so gedacht hat - dieser Gedanke wird im Nachhinein nachgeschoben - oder aber erst von anderen auf dieses Potenzial hingewiesen worden ist. Und diese „Kapazitäten“ (ebd.) seien nun für die „Stipendiatenschaft“ (ebd.), ,hervor[zu]holen und offen und transparent $[\mathrm{zu}]$ machen" (ebd.). Inwiefern dies umgesetzt wird, bleibt an dieser Stelle offen. Stattdessen soll innerhalb der Organisation herausgestellt werden, dass die vorhandene Erstsprache - die scheinbar zu Lasten der Zweitsprache geht - eine nicht weiter ausgeführte Bereicherung für einen nicht näher erläuterten Handlungsspielraum sein kann bzw. das Potenzial hierfür ,,irgendwie“ (ebd.) existiert. Ein Nachteil, der hier an der ethnischen Herkunft festgemacht wird, wird mühsam in ein Potenzial uminterpretiert. Insofern findet sich an dieser Stelle eher Diversity als Kommunikationsinstrument denn als Mittel zur Gewinnmaximierung. Wie letztlich dies genau aussieht, wird aus den Daten nicht deutlich.

Im weiteren Verlauf der Analyse zu den Argumentationskonstellationen zu sozialen Gruppen wird deutlich, was an dieser Stelle bereits durchschimmert: Es gibt nicht jene Fälle, in denen Interviewpartnerinnen und -partner soziale Differenz ausschließlich reproduzieren und andere, die sie schlechthin immer aufwerten usw. Die Vermischung unterschiedlicher Argumente in Hinblick auf soziale Gruppen findet zum Teil zu ein und demselben Projekt oder Thema statt. Es soll überprüft werden, ob nicht dennoch ein Muster zu erkennen ist, die die jeweilige Konnotation (Aufwertung/Reproduktion/ Relativierung/Negierung), die soziale Differenz (Geschlecht, Ethnizität, Klasse etc.) oder eine Kombination von Differenzen und den Benennungskontext (zum Beispiel Geschlecht und Aufwertung) nicht doch in irgendeiner Weise sortieren lässt. Beobachtet werden soll auch, ob eine typische Wirkebene (Mikro-, Meso-, Makro-Ebene) oder ein typisches Handlungsfeld (Bildung, Arbeitsmarkt, Freizeit o.ä.) erkennbar wird.

Negierung sozialer Differenz

Sind in den Abschnitten „Reproduktion sozialer Differenz“ und „Aufwertung sozialer Differenz" soziale Differenzkategorien wohlsortiert und kommen sie eher in maximal zwei Kategorien gleichzeitig vor, werden sie bei der „Negierung sozialer Differenz" komplexer und zahlreicher miteinander verwoben. 
Denn neben dem aufwertenden Gegenentwurf finden sich in den Interviewdaten auch Abschnitte, die soziale Grenzziehungen negieren, also den allgemein als prägsam für soziale Teilhabe wahrgenommenen sozialen Kategorien die Relevanz absprechen. So geht es in Fall V später wie folgt weiter:

Im Grunde halte ich der, den Anknüpfungspunkt Migrationshintergrund für nicht weiterführend, wenn es um sozusagen um Förderung geht. Wenn man nämlich mal sozusagen ja -das wissen Sie- sozio-ökonomisch kontrolliert //Ja.//, sehen wir, dass die, die, die, die sozusagen diejenigen mit Zuwanderungsgeschichte eigentlich auch oft jedenfalls erfolgreicher sind als die Deutschen (V/459-468).

Herr T. hält „den Anknüpfungspunkt Migrationshintergrund“ (ebd.) für die Einschätzung, ob ein Förderbedarf vorliegt oder nicht für „nicht weiterführend“ (ebd.), also für nicht ausschlaggebend in der Ermittlung eines Förderbedarfs. Er gibt an, dass, wenn man ,sozio-ökonomisch kontrolliert“ (ebd.), junge Menschen mit Migrationshintergrund erfolgreicher seien als "die Deutschen“ (ebd.). Er begründet dies mit Erkenntnissen aus dem Bundesbildungsbericht, wonach die Abitur-Quote bei Kindern aus sogenannten „Hartz-IV-Familien“ höher sei, wenn diese einen (türkischen) Migrationshintergrund haben (vgl. V/466 ff.) Aus seiner Sicht führt daher der Migrationsstatus nicht per se zu einem Förderbedarf bzw. geht soweit, dass dieser Aspekt bei der Förderung von jungen Menschen aufgrund der bildungswissenschaftlichen Erkenntnisse keine Rolle zu spielen brauche. Der im Abschnitt „Reproduktion sozialer Differenz“ thematisierten Gleichsetzung von Migration und Armut tritt er hier entgegen. Somit wird an dieser Stelle die Relevanz der sozialen Kategorie „Migration“ im direkten Bezug zur Kategorie „Armut“ im engeren Sinne negiert. Herr T. führt diesen Aspekt weiter aus, indem er sagt:

Also wir fördern auch keine Zuwanderer, die nur aus wohlhabenden Familien kommen. //Ja.// Das wär auch irgendwo Quatsch. //Ja, das stimmt.// Aber schon, aber wenn man ganz ernsthaft ran gehen würde, stellt sich schon die Frage: ist es eigentlich richtig, dass wir osteuropäische Zuwanderer aus jüdischen Familien fördern. Die in einem extremen bildungsgeprägten Elternhaus aufgewachsen sind. Und für die sich eigentlich nie eine Frage sozusagen, auch für die Eltern nie die Frage gestellt hat, ob die Kinder einen anderen Weg gehen könnten als einen akademischen (V/529-540).

Die Negierung findet hier gepaart mit der Binnendifferenzierung der (türkischen) Gruppe dergestalt statt, dass der Aspekt der elterlichen Bildungsaspiration an dieser Stelle mitberücksichtigt wird, die hier allerdings wiederum durch 
Essentialisierung einer bestimmten Migrantengruppe (jüdische Familien) homogenisierend auftaucht. Neben dem Beispiel, dass ein (bestimmter) Migrationshintergrund sich positiv bei gleichzeitig negativer sozio-ökonomischer Disposition auswirkt, kommt eine intersektional neue Perspektive hinzu: wenn der sozio-ökonomische Status positiv ist, müsste ein bestimmter Migrationskontext, nämlich zugezogene deutschstämmige, in osteuropäischen Ländern sozialisierte Menschen jüdischen Glaubens und deren Nachfahren, ein Ausschlusskriterium für Förderung sein. Diese gelten als nicht nur besonders bildungsaffin, anders als Nachfahren der typischen südeuropäischen Gastarbeiterinnen und -arbeiter der 1960er Jahre ist diese Gruppe auch fähig, Bildungsaspiration statuserweiternd einzusetzen, weil ,für die sich eigentlich nie eine Frage [...] gestellt hat" (ebd.) als die akademische Ausbildung ihrer Kinder voranzutreiben (vgl. ebd.). Somit spricht speziell dieser Migrationskontext für eine Aufwertung im sozio-ökonomischen Gesellschaftsgefüge und negiert die Möglichkeit, dass Kinder jüdischen Glaubens - dennoch - Unterstützung brauchen. Ein hoher sozio-ökonomischer Status und andere Migrationskontexte werden hier allerdings nicht in die Argumentation eingeschlossen (,,keine ... nur aus wohlhabenden Familien“ (ebd.)), sondern es bleibt offen, ob zum Beispiel ein italienisches Kind aus wohlhabendem Elternhaus nicht doch „Hilfe“ bräuchte, wo das jüdische Kind offensichtlich keine braucht. Die Argumentation weist auf ein hybrides Verständnis eines sozialen Raums, der nicht nur vertikal nach ökonomischem Status, sondern auch nach familiengeschichtlicher Herkunft verschränkt. Dabei ist nicht die horizontale Trennung nach Lebensstilen oder Milieus gemeint, sondern es wird eine weitere Unterscheidungsebene aufgezeigt, die nach Migrationskontexte differenziert. Dem allgemeinen Trend des Abschnitts über die Argumentationskonstellationen, „Religion“ sei bei der sozioökonomischen Platzierung relevanter als „Migration“ folgt diese Argumentation ebenfalls.

Wieder im Kontext eines Förderprogramms für junge Menschen im Bildungssystem sagt Herr I.:

Um einfach, also auch mit, mit oder ohne Migrationshintergrund ist glaub ich nicht so entscheidend erst mal. Entscheidend ist, dass wir einfach mit den T-Stipendiaten so genannte Bildungsgewinner haben oder erfolgreiche Absolventen unseres Bildungssystems (VIII/218-222).

Der Migrationsstatus der Stipendiatenschaft sei nicht so entscheidend (vgl. ebd.). Der Geschäftsführer der Stiftung formuliert ein anderes Ziel. Er bekräftigt, dass es ,(e)ntscheidend“ (ebd.) für die Stiftung sei, ,,so genannte Bildungsgewinner" (ebd.) zu bekommen und präzisiert die Beschreibung mit ,erfolgreiche Absolventen unseres Bildungssystems“ (ebd.). Das Differenzmerkmal 
„Migration“ tritt hier in den Hintergrund, das Paradigma der kulturellen Angleichung („Integration“) verliert an Bedeutung. Realisieren die jungen Menschen mit Hilfe des Stipendiums einen guten Abschluss, erzielen sie unabhängig von der Herkunft des Elternhauses den Effekt, den die Stiftung möchte, nämlich einen Statusaufstieg oder -erhalt. Dieser „Wunsch“ ist bereits im Laufe der Analyse der Argumentationskonstellationen einige Male aufgetaucht und weist, ähnlich wie im Rahmen des Boundary-making-Ansatzes thematisiert, darauf hin, dass Organisationstypen rhetorische Übereinkünfte erzielen (Lamont/Molnár 2002), die unabhängig von den konkreten Personen abgerufen werden können.

Grundsätzlicher in der Negierung sozialer Differenz beschreibt ein anderer Stiftungsvertreter, Herr U., der seine Ausführungen zur Zielgruppendefinition im Zusammenhang mit der Öffentlichkeitsarbeit der Stiftung trifft.

Immerhin besteht Deutschland aus ungefähr 60, 65 Millionen erwachsenen Bürgern, die wählen können. Und davon, die hatte ich Ihnen ja eben gesagt, sind 45.000, die wir in einem Jahr erreichen nun mal grade nicht viel (IV/697-699).

Aber nicht jetzt irgendwo an, an Menschen, die jetzt, ob sie nun Ausländer sind oder ob sie nur Deutsche sind, da können wir ja dran teilnehmen. Wir sehen, wir sehen uns auch jetzt nicht hier drin bestärkt zu sagen wir machen jetzt ein Angebot für unterprivilegierte Schichten. Wie soll man das denn machen? (IV/721-724)

Die Stiftung erreiche 45.000 Menschen. Vor dem Hintergrund der Gesamtbevölkerung rechnet sich der Programmleiter eine geringe Reichweite aus (eigene Berechnung: ca. 0,073 Prozent). Allerdings fokussiert er allein Personen mit Wahlrecht, was im Umkehrschluss Personen mit ausländischer Staatsangehörigkeit - genauer: sogenannte Drittstaaten-Angehörige - und Kinder unter 16 Jahren bzw. Minderjährige ausschließt. Er führt weiter aus, dass sich die Stiftung nicht zur Aufgabe gemacht habe ,jetzt ein Angebot für unterprivilegierte Schichten“ (ebd.) zu machen. Wie man ein solches Vorhaben umsetzen könnte, ist dem Interviewpartner unbekannt. Er formuliert es als Frage: „Wie soll man das denn machen?" (ebd.). Zuvor hat er bekräftigt, dass alle an den Veranstaltungen teilnehmen können „ob sie Ausländer sind oder ob sie nur Deutsche sind“" (ebd.). Er spricht sozialen Differenzmerkmalen die Besonderheit ab, die ihnen durch Benennung als spezifische Zielgruppe eines Projektvorhabens zuteilwird. Er negiert scheinbar vollständig die Existenz von sozialer Differenz auch unter Zusatz sozialer Ungleichheit, und das auf der gesellschaftlichen Makro-Ebene. Allerdings behält er die Trennlinie zwischen deutschen Staatsangehörigen und Menschen ohne diese bei. Zusammengefasst zeigt sich ein 
„undoing class while doing nationality“. Werden „Ausländer“ (ebd.) ausgeschlossen und die Existenz einer deprivilegierenden sozial niedrigen Herkunft negiert, bleibt im Umkehrschluss die alteingesessene Mittel- und Oberschicht als Zielgruppe übrig.

In den bisherigen Ausführungen wurde deutlich, dass soziale Differenz in den verbalen Darstellungen der Interviewten unterschiedlich konnotiert ist: Mal wurden soziale Differenzmerkmale reproduziert, in einer speziellen Form der Reproduktion zeichnete sich der Umgang mit sozialer Differenz durch Aufwertung aus. In der speziellen Form, die in diesem Abschnitt vorgestellt wurde, wird ein negierender Umgang mit sozialer Differenz sichtbar. Das heißt, sozialer Differenz oder einzelnen ihrer Kategorien wird abgesprochen, für die Programmatik der Stiftung relevant zu sein. Allerdings tritt zu Tage, dass die Negierung immer eingeschränkt oder unter bestimmten Kontextfaktoren erfolgt und nicht die Grundsätzlichkeit aufweist, die die Interviewten illustrieren möchten.

Im nächsten Abschnitt zeigt sich eine Herangehensweise an soziale Differenz, die ebenfalls aus einer intersektionalen Perspektive sehr interessant erscheint und als „Relativierung sozialer Differenz“ bezeichnet werden soll.

\section{Relativierung sozialer Differenz}

In Fall I wird eine weitere Herangehensweise an soziale Differenz ausgeführt, die bisher nicht in dieser Form thematisiert wurde. In Zusammenhang mit der Bekämpfung von Kinderarmut im lokalen Kontext sagt Frau X.:

Zwei Drittel der Kinder, die Leistungen beziehen, haben Migrationsvorgeschichte. Jetzt möchte ich mal nachfragen: Ist denn Kinderarmut ein Thema bei Familien mit Migrationsvorgeschichte? Ist es nicht, nicht ausschließlich! Auch da gibt es eine Menge an Menschen, die es auch geschafft haben, die wirklich gut integriert sind, die auch nicht diese Armutsrisiken haben (I/460-464).

Im Rahmen ihres lokalen Engagements verteilt die Stiftung eine Art Bildungsgutschein an Kinder aus prekären Elternhäusern. Die Geschäftsstellenleiterin bemerkt, dass „(z)wei Drittel“ (ebd.) der Leistungsbeziehenden dieser Gutscheine einen Migrationshintergrund haben, um sodann die rhetorische Frage zu stellen, ob Kinderarmut (tatsächlich) so oft ein Thema in Migrantenfamilien sein könne. Sie beantwortet die Frage selbst damit, dass das „nicht ausschließlich“(ebd.) der Fall sei. Schließlich gebe es auch in dieser Gruppe „eine Menge an Menschen, die es auch geschafft haben" (ebd.), womit ein sozioökonomischer Status gemeint ist, in dem der Lebensunterhalt selbstständig erarbeitet 
werden kann und ein gewisses Mindestmaß erfüllt wird (,nicht diese Armutsrisiken haben“( (ebd.)). Hinzu kommt, dass Frau X. mit dem Begriff der „Integration" vor allem diesen Mindestwohlstand verknüpft. Für gewöhnlich wird in öffentlichen Debatten soziale Integration von Migranten unter dem Aspekt der kulturellen Angleichung diskutiert. Frau X. geht davon aus, dass die Schnittmenge aus „Armut" und „Migrationshintergrund“ kleiner sein müsste, als es aus der geförderten Zielgruppe hervorgeht. Das zeigt sich später deutlich im Fortgang des Interviews, wo sie sich fragt, ob die Multiplikatoren, die die „Fälle“ auswählen, möglicherweise diesen „Migrationsblick“ (I/467) haben. Die Interviewte möchte unbedingt vermeiden, dass die Zusammensetzung der Nutzer (zu zwei Dritteln Migranten) in der öffentlichen Wahrnehmung als Beweis dafür gewertet wird, dass Kinderarmut ein Migrantenproblem ist. Sie übersieht den Zusammenhang, dass „Migration“ ein plausibler Faktor für Kinderarmut sein kann, obwohl ihre Erfahrung zeigt, dass mehr Migrantenkinder als deutsche Kinder das Angebot nutzen. Möglicherweise haben deutsche Kinder bzw. ihre Familien die Möglichkeit, andere Programme oder Unterstützungsleistungen zu erhalten, was an dieser Stelle gar nicht thematisiert wird. Sie relativiert die Lebensverhältnisse also und geht davon aus, dass hier möglicherweise eine Verzerrung vorliegt, die der öffentlichen Wahrnehmung geschuldet ist, der diejenigen unterliegen, die als Multiplikatoren Förderfälle identifizieren. Sie differenziert den Zusammenhang von Förderbedarf, Migration und Armut detailliert in Bezug auf Familien mit minderjährigen Kindern aus. Allerdings negiert sie den Aspekt des Migrationshintergrunds nicht gänzlich, wie es in Fall V stärker zum Tragen kommt, sieht aber auch keinen Zusammenhang zwischen beiden Differenzen. Dennoch denkt Frau X. die relativierende Argumentation hinsichtlich der Differenzkategorien „Migration“ und „Klasse“ etwas später weiter, sodass es wie in Fall V als Negierung gedeutet werden kann:

\footnotetext{
(D)a ist es auch nicht der reine Integrationsgedanke aus Migrationsperspektive, da geht es uns darum: Kinder in Armut sollen am gesellschaftlichen Leben teilnehmen. Da ist es völlig wurscht, aus welchen Zusammenhängen sie kommen aus welchen Ethnien das sind. Da geht es einfach darum, dass diese Kinder Erfahrungen machen müssen, die wichtig sind für ihr Leben (I/473-478)
}

Frau X. unterstreicht, dass es bei dem Vorhaben nicht um ein klassisches Integrationsprojekt geht, in dessen Rahmen Menschen mit Migrationshintergrund gefördert werden sollen. Sie lenkt den Blick auf den Aspekt des Lebensabschnitts: Der Fokus liege stattdessen bei „Kinder[n] in Armut“ (ebd.), die „am gesellschaftlichen Leben teilnehmen“ (ebd.) sollen. Es geht also hier primär um Minderjährige, um heranwachsende Personen und damit indirekt um 
die Förderung von Familien, die sich in prekären Lebensverhältnissen befinden. Alle anderen sozialen Merkmale sind zweitrangig bzw. werden nicht besonders fokussiert. Wie bei dem Stipendienprogramm für Studierende mit Migrationshintergrund wird die Relevanz der Herkunft aus einer Migrantenfamilie für die Ermittlung eines Bedarfs relativiert. Allerdings fokussiert die Interviewpartnerin den ökonomischen Status sehr stark - und relativiert ihn gegenüber allen anderen. Zunächst tritt die Interviewte einem Othering entschieden entgegen, indem sie allerdings, doing class while undoing ethnicity' vollzieht. In vielen Stiftungsprojekten schwingt letztlich der Aspekt der Armutsbekämpfung mit, zumal die Entstehungslogik von Stiftungen hier wiederzufinden ist. Es wird weiter zu prüfen sein, inwiefern zum „doing class“ weitere Kategorien in Wechselbeziehung stehen. Gerade ein Migrationshintergrund, aber auch der der Lebensabschnitt („Familien“) scheinen an dieser Stelle sehr relevant zu sein.

In Fall VI hatte die Stiftungsvertreterin, wie bereits im Abschnitt „Reproduktion" thematisiert, von einer Broschüre erzählt, die auf Grundlage von Veranstaltungsreihen und Interviews mit muslimischen Jugendlichen entstanden ist. Sie vertieft den Sachverhalt (VI/388 ff.), dass es schwierig war, zentrale Ergebnisse auf diese Broschürenform zu reduzieren, obwohl es ein solch „schwieriges und sensibles Thema“ (VI/389) sei. Dabei sollen gezielt plakative Aussagen vermieden werden, die dann „kontraproduktiv“ (VI/390) seien und vermittels derer man in „Verallgemeinerung“ (VI/391) gerate. Im Anschluss daran wurde seitens der Interviewerin nachgehakt, ob neben der Fokussierung von Muslimen in diesem speziellen Projekt auch allgemein andere Zielgruppen genauer ins Auge gefasst wurden, zum Beispiel Frauen. Frau T. entgegnet:

[...] ich kann mir gar nicht vorstellen, dass die das nicht ständig mitdenken. So wie ich auch die Kollegen kenne. Also das ist, ich denke, das ist eine Selbstverständlichkeit. Und es ist ((räuspert sich)) für mich eben so wie ich es hier erlebe auch eine Selbstverständlichkeit, dass man darauf achtet, wenn man, wenn man Podien besetzt (VI/426-430).

Etwas anderes, als dass die Förderung bzw. Beteiligung von Frauen ständig mitgedacht würde (vgl. ebd.), könne sich die Interviewte gar nicht vorstellen. Es sei eine „Selbstverständlichkeit“ (ebd.), dass „man darauf achtet“ (ebd.), dass Frauen auf allen Ebenen der Organisation mitwirken, sowohl als Geförderte wie als Beteiligte. Auf die Idee, Frauen als Zielgruppe überhaupt zu erwähnen, kommt die Interviewte erst auf Nachfrage -und ist sichtlich irritiert, was sich am Wechsel des Sprachgebrauchs durch halbe, nicht ausformulierte Sätzen erkennen lässt, die nicht zum sonst sehr flüssigen Verlauf des Interviews passen. Wird in diesem Interview offenkundig zu Zielsetzungen in Stiftungs- 
projekten weiter oben noch, doing religion and age' betrieben bzw. diese Kategorien reproduziert, setzt an dieser Stelle deren Relativierung ein, dass die Kategorie „Geschlecht“ auf allen Ebenen des Projekts ohne großes Aufsehen mitgedacht werden müsse. Frauen sind dann nicht Fördersubjekte im Projekt, wie andere Personengruppen scheinbar wahrgenommen werden. Sie sind selbstverständlich in der Administration des Projekts, als Input-Geberinnen in den Veranstaltungen und eben als Zielgruppe gleichermaßen relevant und offenkundig vorhanden.

Mit einem letzten Zitat soll der Abschnitt um die Argumentationen zu sozialer Differenz komplettiert werden.

In Fall X verlässt Frau L. die gegenwartsbezogene Betrachtungsweise zu sozialer Differenz in Projekten. Sie führt in einer Art „Zukunftsvision“ die aus ihrer Sicht wichtigen Herstellungsrahmungen sozialer Unterschiede an, die in den politischen wie sozialen Projekten aller möglichen Akteure als Herausforderung anzusehen sind, wenn „Zusammenhalt in der Gesellschaft“ (X/47) erzielt werden soll. Sie tut dies relativ am Anfang des Interviews.

Es gibt das Zuseh-, Zusammenleben von Menschen, die einen unterschiedlichen kulturellen und manchmal auch religiösen Hintergrund mitbringen. Aber es gibt auch viele andere Unterschiede, also Unterschiede im, in den, im Bildungsgrad, in der Art wie Familie erlebt wird. Es gibt geschlechterspezifische Unterschiede. Es gibt nach wie vor Unterschiede zwischen Ostdeutschland und Westdeutschland. Also uns ist es auch wichtig diesen Begriff Vielfalt nicht einzuschränken auf das Thema Integration von Menschen, die hier her integriert sind. Sondern es geht darum die Vielfalt in der Gesellschaft sehr, also in all ihren Facetten zu begreifen. Wir werden auch -grade im Zuge des demographischen Wandels- ein zunehmendes Generationenthema haben mit mehr alten Menschen, weniger jungen Menschen (X/50-60).

Prominent wird „das Zusammenleben von Menschen“ (ebd.) aus unterschiedlichen kulturellen Herkünften benannt (vgl. ebd.), wobei die religiöse Komponente als Teilmenge dessen vorkommt (,manchmal auch religiösen Hintergrund“" (ebd.)). Vielfalt soll aber nicht auf die Personen eingeschränkt werden (vgl. ebd.), „die hier her integriert sind“ (ebd.), also einen Status haben, bei dem sie vormals nicht integriert waren. Daneben, so zählt Frau L. auf, gebe es Unterschiede ,im Bildungsgrad, in der Art, wie Familie erlebt wird“ (ebd.), und weiter "geschlechtsspezifische Unterschiede“ (ebd.) und „nach wie vor Unterschiede zwischen Ostdeutschland und Westdeutschland“ (ebd.). Im „Zuge des demographischen Wandels“ (ebd.) sei das Zusammenleben der Generationen ebenfalls ein Thema, weil es in Zukunft mehr alte und weniger junge Menschen geben werde (vgl. ebd.). Die Interviewte macht einen Rundum- 
schlag durch diverse Kategorien, die neben Unterschieden des Lebensabschnitts und Lebenslaufs mal den Lebensstil, die regionale Herkunft oder askriptive Merkmale ins Zentrum der Betrachtung rücken. Sie eröffnet damit den Blick für eine Zielgruppen-Herstellung, die sehr viele Kategorien auf einmal berücksichtigt und durch Überkreuzung dieser sehr spezifische Zielvorhaben möglich macht. Eine Fokussierung auf einzelne soziale Kategorien nimmt sie als Einschränkung (vgl. ebd.) dieser „Vielfalt der Vielfalt“ wahr, weshalb es als Negierung konkreter sozialer Kategorien zu werten ist. In der Summe schließt der Rundumschlag neben den intersektionalen Überkreuzungen vieler Kategorien, die die statusbezogene Platzierung von Individuen vornehmen, auch die symbolische Grenzziehung mit ein, in der es weniger um soziale Unterschiede, sondern vielmehr um „doing difference while doing difference“ an sich geht.

\subsection{Stiftungen und soziale Differenz}

In 4.3.6 wurden die Benennungskontexte sozialer Differenz durch deutsche Stiftungsvertretende analysiert. Diese waren sortiert nach „Reproduktion“, „Aufwertung“, „Negierung“ und „Relativierung“ sozialer Differenz. Im folgenden Abschnitt sollen die Argumentationsstrukturen zusammengefasst und mit Blick auf die konkreten Differenzkategorien zugespitzt werden.

\subsubsection{Sozio-ökonomischer Status}

Der sozio-ökonomische Status fungiert in zahlreichen Ausführungen als „Leitdifferenz". Damit entspricht die Darstellung weitestgehend den in historischen wie gegenwärtigen Abhandlungen beschriebenen Funktionszuschreibungen von Stiftungen, die die „Ergänzungsfunktion“ (Anheier) zu staatlichen Fürsorgeleistungen ausweisen.

Besonders prominent kommt die Kategorie in zwei unternehmensnahen Stiftungen sowie in einer kommunalen Stiftung zur Sprache. In den Fällen I, V und VIII wird die Relevanz der sozio-ökonomischen Situation der Zielgruppe immer wieder erwähnt und in Wechselbeziehung zu anderen Kategorien diskutiert.

In Fall I erfolgen diese Ausführungen im Rahmen eines Projekts, das die Teilhabe von Kindern an Bildungsaktivitäten durch sogenannte Bildungsgutscheine gewährleisten soll. Durch diese Förderung sollen sie am gesellschaft- 
lichen Leben teilhaben dürfen und in ihren Entwicklungsmöglichkeiten gegenüber Kindern aus wohlhabenderen Familien nicht benachteiligt werden. In Fall I wird „doing class“ betrieben, wobei der Fokus auf den Lebensabschnitt (Kindheit) gelegt wird (siehe unten) und die Wechselbeziehungen zur Kategorie „Migration/Ethnizität“ beleuchtet werden. Es zeigt sich ein „doing class while undoing ethnicity“, da im Laufe des Interviews die ethnische Herkunft der Kinder als zweitrangig dargestellt wird. Alles im allen verweist die Interviewte auf den starken Fokus der Stiftung auf die Klassenlage der Zielgruppe. In diesem Punkt ähneln sich die Äußerungen von Frau X. jenen von Herrn I. in Fall VIII. Auch er möchte wissen lassen, dass es letztlich auf die ökonomische Situation des Elternhauses ankommt, wenn im Rahmen eines Stipendienprogramms für Migrantenkinder Förderung beantragt und zugesprochen wird. Wird in Fall I noch einem Othering durch Ethnizität widersprochen, wird dieses allerdings in Fall VIII vollzogen, da hier der Fokus auf den ökonomischen Status über „Migration“ als eine Art Filterdifferenz erfolgt. Armut und Migration bedingen sich dadurch und werden gemeinsam gedacht, ohne mögliche Erklärungen zu ergänzen. Somit findet sich bei Fall VIII diese Benennungspraxis verschärft (,doing class while doing ethnicity“) vor und weist somit auf eine intersektionale Gleichzeitigkeit hin.

In Fall V nimmt sich der Interviewte nicht der Förderung von weniger privilegierten Zielgruppen an, sondern schließt im Umkehrschluss Personengruppen aus wohlhabenden Kreisen für eine Unterstützung im Bildungserwerb aus. Dies erfolgt im Rahmen von Ausführungen zu einem Stipendienprogramm, das ebenfalls eigens für junge Menschen mit Migrationshintergrund konzipiert wurde. Somit schränkt er die Zielgruppe um zwei Aspekte gleichzeitig ein: „Migration“ mit „Nicht-Wohlstand“. So wird in Fall V ähnlich wie in Fall I „doing class while undoing ethnicity" betrieben.

Allein in einer parteinahen Stiftung (Fall IV) wird der Klassenlage eine Bedeutung für die Stiftungsarbeit explizit abgesprochen. Der Interviewte vollzieht rigoros ein ,undoing class“. Insgesamt haben Interviewte aus parteinahen Stiftungen im Kontext von „Benachteiligung“ weniger zu berichten als alle anderen Stiftungsvertretenden.

In diesem Zusammenhang ist es schwer einzuschätzen, inwiefern weitere Programme und Projekte für die sozio-ökonomische Teilhabe konzipiert wurden, die im Bereich der ,politischen Beteiligung“ angesiedelt sind. Letztlich kann die Befähigung junger Menschen zu politischer Teilhabe (Fall X, unternehmensnah) oder Weiterbildungsmaßnahmen für Soft Skills (Fall IX, politische Stiftung) unter bestimmten Umständen ökonomische Teilhabe durch politisches Mitspracherecht erleichtern oder sogar gewährleisten. Daher sollen sie an dieser Stelle nicht unerwähnt bleiben. 


\subsubsection{Migration/Ethnizität/Religion}

Bereits in den Fällen, die mit der Leitdifferenz ,sozio-ökonomischer Status“ arbeiten, erwähnt, findet sich die Kategorie „Migration/Ethnizität/Religion“ in Wechselbeziehung dazu. So zeigt die Geschäftsstellenleiterin in Fall I unter Verweis auf den hohen Migrantenanteil an durch Bildungsgutscheine geförderten Familien, dass die öffentliche Wahrnehmung dies möglicherweise befeuere. In anderen Fällen werden die Differenzkategorien allerdings ohne große Einschränkungen reproduziert oder stehen allein für sich.

Frau T. führt „,muslimische Jugendliche“ im Rahmen einer selbst durchgeführten qualitativen Studie zu eben dieser Zielgruppe an, aufgrund derer eine Infobroschüre zur Radikalisierungsprävention produziert wurde. Auch diese Zielgruppe, die sich in öffentlichen Debatten wiederfinden lässt, wird durch die Hinzunahme zweier, gleichzeitig eingebrachter Differenzkategorien (Alter bzw. Lebensabschnitt: jung \& Religionszugehörigkeit: muslimisch) präzisiert, wenngleich die Gruppe zahlenmäßig noch sehr groß bleibt. Es handelt sich hier um eine politische Stiftung. Allgemeiner formuliert es in Fall X die Interviewte, wenn sie davon spricht, dass das Verständnis zwischen Muslimen und NichtMuslimen zu fördern sei. Eine weitere Fokussierung wird nicht vorgenommen, sondern es wird ein Stiftungsziel präzisiert, das als ,gesellschaftlicher Zusammenhalt" zusammengefasst wird. Demnach sieht die Interviewpartnerin diesen Zusammenhalt durch ein Nicht-Verständnis zwischen diesen beiden Gruppen in Gefahr. In beiden Fällen wird das Anderssein durch die Differenzkategorie explizit hervorgebracht, es handelt sich daher um ein ,Othering'.

In Fall V wird das einzige Mal die jüdische Minderheit in Deutschland erwähnt, allerdings in Zusammenhang mit einer deutschen Herkunft und einem gleichzeitigen osteuropäischen Migrationshintergrund. Diese wird positiv als bildungsaffine Gruppe aufgewertet; ihr wird ein Bedarf an Unterstützung weitestgehend abgesprochen. Das Förderprogramm mit dem Ziel, Kinder aus Migrantenfamilien zu unterstützen, dient in Fall V auch als Kommunikationsinstrument nach außen, mit dessen Hilfe Vorurteilen in der Bevölkerung begegnet werden solle. Daher wird hier Diversity propagiert. Aufwertend kommen auch „ältere Migranten“ in Fall II, einer unternehmensnahen Stiftung, vor, denen generell eine eigene Autorität zugestanden wird, die deutsche Seniorinnen und Senioren offensichtlich nicht in dieser Ausprägung zu haben scheinen. An dieser Stelle zeigt sich eine Essentialisierung der Zielgruppe.

Im Übrigen streuen sich die Einschätzungen zu Migration und Ethnizität relativ breit in der Benennungspraxis.

In Fall IX (politische Stiftung) erschließt sich die Interviewpartnerin den Migrationskontext über den (Fremd-) Sprachenerwerb von Teilnehmenden in 
den Stiftungsprogrammen. Dabei wird einerseits Stipendiatinnen und Stipendiaten mit Migrationshintergrund ein ergänzender Sprachkurs angeboten, allerdings wenig später mit dem Verweis versehen, dass auch die Erstsprache der Geförderten in irgendeiner Weise ein Potenzial sei, das erschlossen werden müsse. Der Diversity-Ansatz zeigt sich in Fall II durch die Aufwertung älterer Migrantinnen und Migranten, zuvor schon Älterer allgemein, und in diesem Fall IX durch die Potenzialdeklaration migrantischer Teilnehmender, die eine andere Erstsprache als Deutsch aufweisen.

In Fall VIII wird der so benannte Definitionsrahmen „Migrationshintergrund“ bei der Festschreibung der Zielgruppe trotz Bedenken beibehalten, da es auch um (soziale) Integration gehe. Zudem ist mit Verweis auf die Kategorie „ökonomischer Status" das tatsächliche Anliegen der Stiftung, namentlich Talente zu fördern und Armutsbekämpfung zu betreiben, auf diesem Wege leichter zu realisieren. Bereits unter den Ausführungen zum „sozio-ökonomischen Status“" wurde dieses Vorgehen als „doing class while doing ethnicity“ erwähnt.

\subsubsection{Lebensabschnitt/Alter/Lebenslage}

So sehr Deutungen und Interpretationen zu den Themen „Armut“ oder „Ethnizität" divergieren, so eindeutig arbeiten Stiftungen mit ähnlichen Zielgruppen, wenn es um den Aspekt „Alter“ oder „Lebensabschnitt“" geht. Wie aus den vorangegangenen Ausführungen hervorgeht, beschränken sich Förderprogramme zumeist auf Seniorinnen und Senioren, Familien mit minderjährigen Kindern sowie junge Menschen, die sich noch in Ausbildung befinden oder sich noch weiterbilden möchten. Seniorinnen und Senioren werden in Fall I als „Potenzial" diskutiert, in Fall X wird ihre gesellschaftliche Teilhabe als Herausforderung der Zukunft betrachtet (Fall X/59-60). Diese Herangehensweise unterscheidet sich von den Förderzielen zu Kindern, Familien und jüngeren Menschen. Diese werden als beeinflussbar für sozialen Aufstieg oder politische Teilhabe wahrgenommen, ein ökonomischer Nachteil von Familien mit insbesondere kleinen Kindern wird besonders problematisiert (Fall I und Fall VII, beide mit starkem lokalen Fokus).

Allen drei Zielgruppen im Bereich des Lebensabschnitts wird demnach ein „Mangel“ zugesprochen: bei Seniorinnen und Senioren ist dies soziale Teilhabe (Fall II) oder implizit der Mangel an körperlicher Befähigung (Fall X). Bei Familien bzw. Kindern wird ein ökonomischer Nachteil wahrgenommen, der für den individuellen Lebenslauf als besonders fatal gilt. Junge Menschen in Ausbildung teilen sich in zwei Gruppen: diejenigen, die Unterstützung brauchen, weil diese durch ihr Umfeld nicht gewährleistet werden kann, obwohl sie unterstützungswürdig sind (,talentiert“ Fall VIII/116). Die andere Gruppe 
wird eher umworben, gilt als Potenzial und fungiert möglicherweise zu einem späteren Zeitpunkt als Multiplikatorin (Fall X/276 ff.). „Elitenförderung“ wird letztlich in einigen Stiftungen angesprochen (Fall VII/309 f.; implizit in den Fällen, die Stipendienprogramme unterhalten, also seitens der politischen Stiftungen sowie in den Fällen V und VIII) und weist eher auf den Wirkungsbereich der Stiftung hin, der sie als politische Akteurin ausmacht.

\subsubsection{Geschlecht}

Wie anhand Fall VI veranschaulicht, scheint Förderung aufgrund des Geschlechts in den Stiftungen kaum eine Rolle zu spielen. Frauen oder Männer werden als konkrete Zielgruppe bei keiner Gelegenheit von selbst erwähnt, sondern kommen in Fall VI nur auf Nachfrage zur Sprache, wobei die Frage sichtlich Irritationen über diese Selbstverständlichkeit auslöste. Dafür weist bereits in Fall II Frau I. darauf hin, dass man zur Gewährleistung eines guten Austauschs Podien usw. immer geschlechterausgeglichen besetzen würde (Diversity, siehe oben). In Fall III/17 ff. führt der Interviewte zur Zukunftsaufgabe der „Frauengleichstellung“ an, die bei statistischen Daten wie Gehaltsvergleichen oder der Verteilung von Mandatsträgern immer wieder auffalle. Auch dies verbleibt auf der Makro-Ebene von Erklärungsmustern und wird nicht im Rahmen von Stiftungshandeln selbst erwähnt. Eine offensichtliche Geschlechterdiskrepanz in den Führungsetagen der Stiftungen selbst kommt nicht zur Sprache. 


\section{Fazit}

\subsection{Rückblick: Standortbestimmung der vorliegenden Studie}

Lange galten Stiftungen als blinde Flecken der Forschungslandschaft. In besonderem Maße trifft dies auf Analysen zu, die empirisch qualitativ zu ihnen forschen und auf diese Weise die operative Arbeit und die gesellschaftliche Verortung als Sonderform zivilgesellschaftlicher Organisationen in den Blick nehmen. Diese werden allmählich angegangen (Anheier u. a. 2017a, 2017b, 2017c; Adloff 2002; Heisterkamp 2014; Striebing 2017; Klaassen 2016; von Reden 2015; Campenhausen 2015; Strachwitz 2016; Strachwitz 2015b; Strachwitz 2007). Als Sonderform werden sie bezeichnet, weil Stiftungen unterschiedlichen, zuweilen widersprüchlichen Anforderungen genügen möchten. Dazu zählt der Wunsch, gemeinwohlorientiert, interessenartikulierend und exklusiv zugleich zu sein. Als interessante Erkenntnislücke erwies sich hier die Frage, wie in der operativen Arbeit von Stiftungen soziale Differenz hervorgebracht wird. Die Beantwortung in dieser Arbeit erfolgte, indem analysiert wurde, wie operative Stiftungen ihre Projekte ins Leben rufen, wie sie diese angehen und welches gesellschaftliche Selbstverständnis sich durch sie offenbart.

Um das Forschungsfeld genauer zu bestimmen, wurde in Kapitel 2 erörtert, was Stiftungen ausmacht und wie sie sozialwissenschaftlich einzuordnen sind. Da die vorliegende Studie besonders sogenannte operative große Stiftungen erfasst, gehörte dazu die Diskussion des in der Politikwissenschaft häufig aufgeworfenen Problems eines Demokratiedefizits von demokratisch nicht legitimierten Organisationen. Hierunter fällt auch die Stiftungsarbeit, da namentlich operative große Stiftungen in die öffentliche Sphäre hineinwirken. Es lassen sich einige einschlägige Arten von Stiftungen unterscheiden: Es handelt sich um Organisationen, die auf einen oder mehrere Stifter zurückgehen, die vormals Vermögen akkumuliert haben und dieses im Rahmen formaler Strukturen ideell anlegen möchten (insbesondere: unternehmensnahe Stiftungen). Die Gründungsgeschichte muss nicht unbedingt auf eine natürliche Person zurückgehen. Sie kann auch durch eine parteinahe Gruppe (politische Stiftung), eine öffentliche Einrichtung (kommunale Stiftung) oder durch eine Glaubensrichtung (konfessionelle Stiftung) ins Leben gerufen worden sein. Ihr Verhältnis zu staatlichen Institutionen ist regelmäßig ambivalent: Sie werden zur Realisierung von gemeinwohlorientierten Vorhaben gebraucht, um aufgrund des staatlichen Informationsmonopols Fürsorge-Bedarfe zu ermitteln. Gerade politische Stiftungen erhalten hohe Summen aus der öffentlichen Hand. Dennoch 
sind alle Stiftungen gleichzeitig dazu angehalten, nicht als Konkurrenz zu staatlichen Stellen aufzutreten, stabilisierend zu wirken und sich bestimmten rechtsstaatlichen Mindeststandards zu unterwerfen, um ihr Demokratiedefizit nicht weiter zu verschärfen. In wissenschaftlichen Beiträgen wird mit Blick auf Stiftungen der Wunsch geäußert, sie könnten die Nachhaltigkeit langfristiger gesellschaftlicher Herausforderungen unter den Bedingungen unsicherer Konjunkturen politischer Gemengelagen sicherstellen (Adloff 2004) und Schwächen der (Sozial-) Staatlichkeit kompensieren (Zimmer 2002b).

Wie in Kapitel 3 ,Soziale Differenz“ ausgeführt wurde, war es das Hauptanliegen der Arbeit, die Benennungspraxis sozialer Grenzmarkierungen von Stiftungen als zivilgesellschaftliche Akteure vor dem Hintergrund heterogener Gesellschaftsstrukturen und sozialstrukturell ungleicher Teilhabechancen zu erörtern. Es musste ein Weg gefunden werden, den nicht eindeutig festgelegten Ausdruck der sozialen Differenz für die empirische Arbeit greifbar zu machen. Dabei galt es sich der Herausforderung zu stellen, dass nicht per se definiert ist, was soziale Differenz sein soll bzw. welche soziologischen Phänomene damit erfasst werden. Die Lösung lag darin, eine synoptische Zusammenstellung einschlägiger Ansätze zu einem Analyserahmen zu verdichten. Dieses Vorgehen hat sich in zwei vorangegangenen Studien bewährt. Die Autorin einer der beiden Studien geht in ihrer Forschungsfrage sehr ähnlich wie die vorliegende Untersuchung vor. Es handelt sich um die Analyse der habituellen Konstruktion sozialer Differenz am Beispiel von Wohlfahrtsorganisationen, veröffentlicht 2008 von Sonja Kubisch. Dem konstruktivistischen Paradigma dieser Arbeit folgend, soziale Differenz sei etwas, das hergestellt wird, wurde ein Filter aufgesetzt. Die Vorstellung der sozialkonstruktivistischen Ansätze mit dem Un/doing-difference-Ansatz rückte die Leitdifferenz „Geschlecht“ in den Fokus. Wird nach West/Fenstermaker (1995) eine ganze Anzahl von Differenzmerkmalen im Alltag beständig reproduziert, illustriert Hirschauer in seinem Un/doing-Ansatz, dass Kategorien in bestimmten Fällen ,ruhen“ können. Die Doing-Ansätze sind auf einer Mikro-Ebene angesiedelt. Sodann wurden die Ansätze erörtert, die die Benennung bzw. Vermengung von Differenzmerkmalen (Migration, Geschlecht, Klasse uvm.) in den Fokus rücken. Im kulturwissenschaftlichen Forschungsprogramm finden sich diverse Hinweise. Während das Boundary Making relativ abstrakt die Wechselbeziehung der strukturellen Ungleichheit und der symbolischen Zuschreibung im Rahmen sogenannter sozialer Einheiten diskutiert, wird bezüglich des Othering vehement auf die diskursive Grenzziehungsleistung hingewiesen. Für den vorliegenden Forschungskontext wurde eine fehlende empirische Fundierung festgestellt. In einem weiteren Ansatz wurde aus einer post-kolonialen Betrachtung heraus auf die Hybridität sozialer Räume hingewiesen, die die besondere Mischform kul- 
tureller Praktiken von ethnischen Minderheiten ins Blickfeld rücken. Die deutsche Postmigrationsforschung kritisiert daran anschließend das dogmatische Verständnis der gegebenen Migrationsforschung als Angleichungsforschung migrantischer Minderheiten. Auch für die Postmigrationsforschung wurde schließlich konstatiert, dass nicht genau beantwortet werden kann, wie die Neuausrichtung und empirische Umsetzung künftig gelingen könne. Beim ungleichheitsorientierten Ansatz Intersektionalität sollen über Geschlecht die Kategorien Klasse und Ethnizität zu einem Modell verschränkt werden. Gleichzeitig formuliert der Ansatz ein Forschungsprogramm, das als Heuristik zu verstehen ist. Im Rahmen der als Managementkonzept bekannten Diversity wird Offenheit gegenüber Menschen propagiert, die hinsichtlich gewisser Merkmale (Geschlecht, Migrationsstatus, Ethnizität, Religion, körperliche Beeinträchtigung, sexuelle Identität) vom vermeintlichen Arbeitnehmer-Idealtypus abweichen. Dabei wird vergessen, Klassismen mitzudenken.

Um schließlich die Ansätze synoptisch gegenüberzustellen, wurden sie nach relevanten Kriterien sortiert und in drei Punkten verdichtet, die im $\mathrm{Zu}$ sammenhang mit der Herstellungsweise sozialer Differenz wichtig sind. Die jeweiligen Ungleichheitsdimensionen sind zunächst in der Analyse ihrer potenziellen Bedeutung für die Benennungspraxis gleichwertig zu behandeln (Geschlecht, Ethnizität, Klasse, weitere). Der Analyse sollte keine „Leitdifferenz" vorgeschaltet werden, wie es in den theoretischen Implikationen häufig vorkommt. Dies würde auch die Rekonstruktion einer „Leitdifferenz“ im empirischen Material erschweren. Im Umkehrschluss wurden die Ansätze bewusst auch auf Differenzkategorien bezogen, die eigentlich nicht in den jeweiligen Ansätzen explizit verhandelt werden. Als nächstes ist die Beziehungsherstellung zwischen der Organisation und Zielgruppen bzw. Kooperationspartnern in den Blick zu nehmen. Das gestaltet sich im Rahmen von Stiftungsforschung mit einer zivilgesellschaftlichen Verortung als recht komplex, da, wie in Kapitel 2 ausgeführt, Stiftungen ambivalente Funktionen zu erfüllen versuchen. Es waren daher besonders Selbstdarstellungen der Repräsentantinnen und Repräsentanten einzelner Stiftungen genau zu betrachten, um in Bezug auf Rollendefinitionen auch die Beziehungsherstellung zu gesellschaftlichen Gruppen zu analysieren. Als letztes wurde bei der synoptischen Gegenüberstellung festgestellt, dass die Benennungspraxis hinsichtlich der Konnotation einer sozialen Differenzkategorie sowie die Wechselbeziehungen zwischen den Differenzmerkmalen gleichzeitig zu analysieren war. Als Hauptanliegen wurde formuliert, ob in der Analyse von Differenzmerkmalen, Wirkebenen und Benennungspraxis ein Muster erkennbar wird.

Wie die empirischen Daten erhoben wurden, wie sie zu analysieren waren und was als Ergebnis zu Tage getreten ist, wurde in Kapitel 4 erörtert. Zunächst 
wurde geklärt, dass die Auswertung der Experteninterviews in wissenssoziologischer Tradition nach Meuser/Nagel (1991, 1995, 2005, 2009) erfolgen soll. Analog zu Meuser/Nagel war davon auszugehen, dass auch bei Experten ein „vortheoretisches Erfahrungswissen“ (Meuser/Nagel 2009: 470) existiert, das es zu rekonstruieren galt.

Schließlich wurden die zehn Experteninterviews mit elf Expertinnen und Experten (ein Interview im Tandem) zwischen August 2011 und September 2012 in verschiedenen Städten Deutschlands durchgeführt. Vier der operativen Stiftungen galten als sogenannte politische Stiftung, fünf waren unternehmensnah. Bei einer Stiftung handelte es sich um eine des kommunalen Typs. Die Positionen der Interviewten in der jeweiligen Organisationshierarchie waren unterschiedlich: von der jungen Projektleiterin über den erfahrenen Geschäftsführer bis hin zum etablierten Vorstandschef waren in der Stichprobe unterschiedliche Personen vertreten. Der Leitfaden bestand aus drei Themenblöcken: Im ersten Block wurden allgemeine Fragen zur Tätigkeit in der Stiftung, zum Leitbild und zu favorisierten Projekten gestellt. Im nächsten Block erfolgte die Abfrage der operativen Projekterfahrung, in der es darum ging zu untersuchen, warum bestimmte Projekte oder Vorhaben ins Leben gerufen wurden, wie schwierig oder einfach ihre Umsetzung war und wie die Durchführung sonst erlebt wurde. Im dritten und letzten Block ging es um die Projektwirkung und Zielgruppendefinition. Es wurde gefragt, welche gesellschaftliche Wirkung mit dem Vorhaben erhofft und warum genau bestimmte - vorher von den Befragten benannte - Zielgruppen ausgewählt wurden.

In der Selbstdarstellung zeichneten die Interviewten ein Bild, das Stiftungen als objektive, (selbst-) wirksame, gemeinwohlorientierte, innovative und besonders bemerkenswerte Organisationen illustrierte. In Abgrenzung zu staatlichen Akteuren wurde ein starker lokaler Fokus, die unbedingte Stärkung des Bürgers bzw. der Bürgerin als mündige Individuen sowie die Artikulation von Gesellschaftsvisionen deutlich, die aus Sicht der Interviewten als „ungelöst“ oder „Zukunftsaufgabe“ wahrgenommen wurden, deutlich. Die Wechselbeziehung zwischen Staat und operativem Stiftungswesen zeichnete sich durch eine Haltung aus, die zunächst Kooperationen unumgänglich erscheinen ließ. Aufgrund des staatlichen Informationsmonopols bezüglich sozialer Bedarfe, der hohen rechtsstaatlichen Ansprüche nach Gleichheit und letztlich der hohen Summen, die besonders politische Stiftungen durch die öffentliche Hand erhalten, ist der Staat zudem für das Stiftungswesen unverzichtbar. Die Priorität in Hinblick auf ihre Rolle als gemeinwohlorientierte Organisationen zeigte sich auch bei der Analyse der Benennungskontexte sozialer Differenzkategorien. Die Erwähnung des sozio-ökonomischen Status dominierte insbesondere bei der Beschreibung von Fördermaßnahmen, wenngleich eine vielschichtige Wechselseitigkeit mit der Kategorie „Ethnizität/Migration“ festzustellen ist. 
Letztere wird zum Teil als Filterkategorie in den Vordergrund gedrängt, obwohl sie nicht direkt gemeint ist und in der Auswertung gehäuft als Beispiel für eine „Negierung“ oder „Relativierung“ sozialer Differenz bezeichnet wurde. Zum Teil reflektierten die Expertinnen und Experten diesen Vorgang allerdings als Kommunikationsstrategie oder als „Problem“, das der öffentlichen Wahrnehmung anderer Mitwirkender in den Projektkontexten geschuldet sei. Neben vielschichtigen Argumenten in Hinblick auf soziale Differenz nahmen die Expertinnen und Experten auch Stellung zu ihrer Rolle gegenüber dem Staatswesen deutlich ab. Es ist eine Beziehung, in der Stiftungen staatlichen Institutionen nicht blind folgen, sie nicht imitieren, aber auch nicht in Frage stellen und gegebenenfalls sogar unterstützen. Dadurch entsteht ein interessantes Spannungsfeld, dessen theoretische Fundierung noch nicht abgeschlossen ist.

\subsection{Ergebnis: Ambivalenz der Organisationsform Stiftung und Vielschichtigkeit der gesellschaftlichen Wirkebene}

Die empirischen Befunde dieser qualitativen Studie speziell zu großen, bekannten und operativ agierenden Stiftungen ermöglichen einerseits eine speziellere Einordnung in den gesellschaftlichen Kontext sowie andererseits die Systematisierung verbaler Darstellungen von Akteuren hinsichtlich der Herstellungsweise sozialer Differenz.

Zur gesellschaftlichen Verortung von Stiftungen

Akteure und Akteurskonstellationen, die im Umfeld der Stiftung erwähnt werden, sind auf unterschiedlichen Ebenen mit verschiedenen Funktionen versehen. Zum einen werden Individuen bzw. Bürgerinnen und Bürger als Leistungsempfangende benannt, die in Projektvorhaben als Zielgruppe gehandelt werden. Als Kooperationen mit ,Eliten “ und zivilen Repräsentationen verstanden, zeigt sich bei Stiftungen der Wunsch nach gesellschaftlicher Einflussnahme als politische Akteurinnen. Dergestalt können die Selbstdarstellungen unter gewissen Gesichtspunkten sortiert werden. Besonders herauszustellen ist hier, dass Repräsentierende von Stiftungen immer wieder deutlich gemacht haben, dass ihre Organisation selbst, aber auch Stiftungen allgemein eben ,anders" seien und damit implizit ein Alleinstellungsmerkmal gegenüber Wohlfahrtsorganisationen, staatlichen Institutionen, politischen Parteien, Vereinen und Verbänden artikulierten. Sie inszenieren sich im Vergleich zu politischen Parteien als objektiver, in Abgrenzung gegenüber sozialen Einrichtungen als 
politisch einflussreicher und gegenüber staatlichen Institutionen als innovativer und trotz allem gemeinwohlorientiert. Ihr Alleinstellungsmerkmal führen sie dabei zentral auf ihre Bedeutung namentlich im lokalen Kontext zurück, die es ihnen ermöglicht, gesellschaftliche Verhältnisse sehr differenziert zu beobachten. Das lokale Umfeld bildet auch in vielen Fällen den identifikatorischen Referenzrahmen - und nicht etwa Deutschland als Nationalstaat. Gleichwohl formulieren sie Normvorstellungen zum gesellschaftlichen Zusammenleben, die über den lokalen Kontext hinausgehen und neben der nationalen auch eine internationale Ebene, etwa den europäischen Kontext, miteinschließen kann.

Gegenüber staatlichen Institutionen ist das Verhältnis besonders komplex und dabei als ambivalent zu bezeichnen: neben der klaren Abgrenzung zu dieser Sphäre weisen die Stiftungsvertreterinnen und -vertreter auf diverse Rollen hin, die sie wechselseitig staatlichen Institutionen gegenüber einnehmen. Als Kooperationspartner fungieren staatliche Stellen demnach in erster Linie bei der Ermittlung von Bedarfen der sozialen Fürsorge. Es gibt Projektvorhaben, die durch die öffentliche Hand kofinanziert werden. Stiftungen entdecken dem Anspruch nach frühzeitig Versorgungslücken des Sozialstaats, da sie in lokale und kommunale Strukturen eingebunden werden. Dadurch geben sie an, einem Fürsorge-Anspruch zu genügen, nämlich anders als der Staat ,nah am Menschen“ zu sein. Gleichzeitig soll der Staat auch Profiteur von Stiftungen sein können, wenn er Dienstleistungen wie Mediationsangebote bei lokalen Konflikten oder Kompetenzen aus Aufbauarbeiten für seine Einrichtungen annimmt. Reduziert man dieses Bild, das die Expertinnen und Experten in den Interviews zeichnen, auf seine Kerngestalt, offenbart sich die Vorstellung eines schwerfälligen, altmodischen und trägen Staatswesens, dem Eigeninitiative und bürgerliche Wachsamkeit entgegenzusetzen seien. Trotz dieses ambivalenten Bildes wird der Staat als unverzichtbar für die Realisierung der Zielvorhaben angesehen, da er im Gegenzug - anders als private Organisationen - Ansprüchen wie dem Gleichheitspostulat und hohen rechtsstaatlichen Standards genügen kann.

So ambivalent das Verhältnis zum Staat dargestellt wird, so eindimensional scheint indes die Wechselseitigkeit, mit der die (mediale) Öffentlichkeit wahrgenommen wird. So werden Themen aus der öffentlichen Diskussion herausaufgegriffen, auf die Projektvorhaben aufgebaut werden. Die Stiftungsvertreterinnen und -vertreter formulieren auf allen Hierarchieebenen den Wunsch, in diese öffentliche Sphäre hineinwirken zu wollen, ohne konkret zu werden. Dadurch vervollständigen sie ein Bild davon, wie Stiftungen neben aller Gemeinwohlorientierung, innovativer Pionierarbeit und trotz aller Organisationsansprüche der Objektivität auch herkömmliche politische Akteurinnen sein können. In welchem Verhältnis diese beiden Komponenten zueinanderstehen, 
dürfte von Stiftung zu Stiftung stark variieren und müsste in Einzelfallanalysen geklärt werden.

Als programmatisches Ziel erwähnen alle Stiftungsvertretenden, gesellschaftlichen Zusammenhalt stärken, herstellen oder bewahren zu wollen. Neben der Moderation von sozialer Vielfalt, die diesem Anspruch als Herausforderung entgegengestellt wird, funktioniert aus der Stiftungsperspektive das Wechselspiel von staatlichen Institutionen, Bürgerinnen und Bürgern sowie zivilen und zivilgesellschaftlichen Organisationen durch die Stärkung von Bürgerkompetenzen. Unter derartigen Maßgaben steht staatliche Herrschaft nicht zur Disposition, wohl aber werden ihre Schwachstellen auf Grundlage von Ansprüchen nach Teilhabe und Einfluss kompensiert. Somit stellen sie sich als Organisationen bewusst zwischen den Staat und den individuellen Bürger. Bezogen auf die individuelle Unterstützungsleistung offenbaren sich mehrere Ebenen: Als soziale Akteurinnen setzen Stiftungen gemeinwohlorientierte Projekte um. Um allerdings das Demokratiedefizit ein Stück weit zu kompensieren, stellen sie die demokratische Teilhabe zu differenzierender Individuen in den Vordergrund. So ist die Stärkung der Bürgerbeteiligung genauso zentral wie die Schaffung und Administration bestimmter Netzwerke und die Interessenartikulation im Rahmen singulärer Veranstaltungen. Auch über das Wirken in die Mikro-Ebene wird ein wechselseitiges Verhältnis zu staatlichen Institutionen manifestiert: Von der Entlastung durch mündige Bürgerinnen und Bürger, die Probleme in die eigene Hand nehmen, bis hin zum unbequemen Meinungsmacher, der Missstände offen anprangern kann und dafür eine Plattform geboten bekommt. Damit Bürgerinnen und Bürger Interessen artikulieren können, nimmt daher der Punkt der „Elitenförderung“ einen prominenten Platz in den Ausführungen der Interviewten ein.

\section{Zur Herstellungsweise sozialer Differenz}

Bei der Erarbeitung der Handhabe zur Herstellungsweise sozialer Differenz zeigte sich, dass die empirischen Daten sehr unterschiedliche Perspektiven auf den Umgang mit sozialer Differenz liefern. So wurde durch die Aufarbeitung der Daten gezeigt, dass konkrete Differenzmarkierungen unterschiedliche Kontexte haben können. Die Interviewten reproduzierten zum einen soziale Differenz in einer zum Teil intersektionalen Überkreuzung, wie sie in öffentlichen Debatten vorzufinden ist. Dieser Vorgang wurde als „Reproduktionsklassiker" vorgestellt. Daneben offenbarten sich unterschiedlich starke Fokussierungen sozialer Differenzkategorien, die mal bewertend (Aufwertung), mal in Frage stellend (Negation) und mal relativierend (Nivellierung) zu Tage treten. Bei der Rekonstruktion der Beziehungswahrnehmung zu sozialen Gruppen 
zeigte sich in den Argumentationskonstellationen, wie Stiftungen gezielt bestimmte Gruppen sowohl aus einem gemeinwohlorientierten Ansinnen heraus unterstützen (Mikro-Ebene), aber auch als Kommunikationsinstrumente verwenden („Erfolgreiche Migranten als Vorbild/Potenzial“ usw.) (Meso-Ebene). Im Falle der Kommunikationsarbeit war eine schillernde Funktion von Stiftungen zu erkennen, die zwischen einer Sozietät mit Objektivitätstendenz und einem politischen Akteur mit Tendenz zu Partikularinteressen hin und her wechseln kann. Als politischer Akteur treten Stiftungen auch dann auf, wenn sie über ihr Wirken auf der gesellschaftlichen Ebene (Makro-Ebene) berichten.

Tabelle 4: Konzeption soziale Differenz und Bezug zu empirischen Daten

\begin{tabular}{|c|c|c|}
\hline Ebene | Ansatz & Quitessenz & Bezogen auf die empirischen Daten \\
\hline $\begin{array}{l}\text { Mikro } \\
\text { ((un/)doing } \\
\text { difference; } \\
\text { Intersektionalität } \\
\text { nach Degele/ Win- } \\
\text { ker 2009, Anthias } \\
2005)\end{array}$ & $\begin{array}{l}\text { Verschränkung verschie- } \\
\text { dener Differenzmerkmale, } \\
\text { die gleichzeitig wirken; } \\
\text { Kategorien können „ruhen" }\end{array}$ & $\begin{array}{l}\text { Paradigma: „Menschen fördern und unterstützen } \\
\text { Fall X: Politische Teilhabe Jugendlicher } \\
\text { (doing age/youth) } \\
\text { Fall IX: Deutsch als Zweitsprache bei Bildungsgewinnern } \\
\text { (doing ethnicity while undoing class) } \\
\text { Fall VIII: Stipendium fur Migrantenkinder } \\
\text { (intersektionale Gleichzeitigkeit von Migration } \\
\text { und Armut) } \\
\text { Fall VII: Frühe Förderung von armen Familien } \\
\text { (doing age and doing class, intersektionale } \\
\text { Gleichzeitigkeit von Armut/ Schicksal und Elternschaft) } \\
\text { Fall V: Stipendium für Migrantenkinder } \\
\text { (doing class and age while undoing ethnicity) } \\
\text { Fall IV: Keine Zielgruppe mit Einschränkung von } \\
\text { Personen ohne Wahlrecht } \\
\text { (undoing class while doing nationality) } \\
\text { Fall I: Bildungsgutscheine für Familien } \\
\text { (undoing ethnicity while doing class and age) }\end{array}$ \\
\hline $\begin{array}{l}\text { Meso } \\
\text { (Diversity) }\end{array}$ & $\begin{array}{l}\text { Wertschatzung von und } \\
\text { Profitmaximierung durch } \\
\text { soziale Vielfalt }\end{array}$ & $\begin{array}{l}\text { Paradigma „Potenzial aufzeigen und nutzen" } \\
\text { (Fall II: Alter \& Migration; Fall V.: Migration \& Alter; Fall } \\
\text { VI: Geschlecht; Fall VII: Migration \& Klasse; Fall VIII.: } \\
\text { Migration \& Klasse; Fall IX: Alter) }\end{array}$ \\
\hline $\begin{array}{l}\text { Makro } \\
\text { (Boundary making; } \\
\text { Othering; Hybridi- } \\
\text { tät; Intersektionali- } \\
\text { tät nach Crenshaw } \\
1988, \text { Klinger/ } \\
\text { Knapp) }\end{array}$ & $\begin{array}{l}\text { Soziale und symbolische } \\
\text { Grenzziehung; diskursive } \\
\text { Differenzmarkierung; Ver- } \\
\text { schränkung von Differenz- } \\
\text { merkmalen, die soziale } \\
\text { Ungleichheit markieren }\end{array}$ & $\begin{array}{l}\text { Paradigma: „Soziale Zusammenhänge erklären und } \\
\text { sozialen Zusammenhalt stärken“ } \\
\text { Fall VI: Studie zu muslimischen Jugendlichen } \\
\text { (boundary making, Othering) } \\
\text { Fall X: Verständnis zu Muslimen fördern } \\
\text { (boundary making, Othering) } \\
\text { Fall III: Veranstaltungsreihe "Der deutsche Traum }{ }^{\circ} \\
\text { (Postmigration) } \\
\text { Fall VII: gesellschaftliche Vielfalt moderieren } \\
\text { (intersektionale Mehrdimensionalität) } \\
\text { Fall X: Vielfaltsdimensionen als Zukunftsaufgabe } \\
\text { (intersektionale Mehrdimensionalität) } \\
\text { Fall V: Stipendium nicht an wohlhabende Migrantenkin- } \\
\text { der aus bestimmten Kontext (hybrider Raum, ethnische } \\
\text { und religiöse Subgruppen mit unterschiedlicher } \\
\text { intersektionaler Verschränkung zur Klassenlage) }\end{array}$ \\
\hline
\end{tabular}

(Quelle: Eigene Darstellung) 
Diese drei Ebenen werden in Tabelle 4 analog zum erstellten Analyserahmen aus Kapitel 3.4 erfasst. Die entsprechende Benennungspraxis wird diesem wie folgt zugeordnet:

Die unterschiedlichen Benennungskontexte wurden in Bezug auf einschlägige Differenzkategorien erschlossen. In der Zusammenfassung der Argumentationskonstellationen zeigte sich unter 4.4, dass bei der Zielgruppendefinition der Stiftungen der sozio-ökonomische Status der zu Fördernden eine wichtige Rolle spielt und sich andere Kategorien an diesem „orientieren“. Soll die Herstellungsweise sozialer Differenz für die Mikro-Ebene skizziert werden, so sehen Stiftungen eine große Aufgabe darin, soziale Randgruppen zu fördern. Das ist nicht neu. Die Fürsorgeleistung, die Stiftungen erfüllen möchten, werden in erster Linie durch den Fokus der Klassenlage (arm bzw. nicht reich) und des Lebensabschnitts (in Ausbildung, Familienphase) gewährleistet. Es zeigt sich, dass Interviewte diese unterschiedlichen Differenzmerkmale ,jonglierend“ handhaben können, da sie durch eine intersektional verfeinerte Zielgruppendefinition konkrete Differenzen nivellieren oder ihnen sogar die Relevanz für die sozio-ökonomische Platzierung gänzlich absprechen. Damit betreiben sie nicht selten ein „undoing difference“, weil sie merken, dass es bei der Fürsorge nicht um das vordergründige Merkmal (Migration/Ethnizität), sondern eigentlich doch um die Klassenlage geht. Haben zu Fördernde den ökonomischen Anschluss an die Mehrheit geschafft oder haben sie diesen schon immer gehabt, fallen sie aus dem Zielgruppenraster der Stiftung und gelten als eingegliedert. So klar dieser Zusammenhang in einigen Fällen reflektiert wird (Fall I und Fall V), so diffus wird er in einigen anderen Fällen (Fall VIII und IV) diskutiert. Die Zielgruppendarstellung erfolgt nicht kausal erklärt im Zusammenhang von Armut, Migration und Lebensabschnitt. Vielmehr wird die implizit als „Hauptziel“ angesprochene Leitdifferenz „Klassenlage“ oder „Familiensituation“ nur tangiert, um das Augenmerk auf die scheinbar ,eindeutigere“ und besser kommunizierbare Kategorie „Migrationshintergrund“ zu richten.

Auf der Meso-Ebene dominiert das Vorhaben der Stiftungen, Paradigmenwechsel anzustoßen, die in Zusammenhang mit bestimmten, deprivilegiert geltenden Personengruppen in der öffentlichen Wahrnehmung beobachtet werden. Dabei sollen Talente, Potenziale oder sonstige „Förderwürdigkeiten“ entdeckt, erkannt und zum „Gegenbeweis“ präsentiert werden. Gleichzeitig ist die „Reproduktions-Falle“ sozialer Differenz aktiv, die zum Teil auch als solche von den Expertinnen und Experten selbst reflektiert wird. Letztlich geht es darum, wie im Diversity-Management üblich, Positives nach außen zu kommunizieren, um einen Mehrwert zu generieren, der im Falle von Stiftungen nicht monetärer Natur sein muss. 
Schließlich zeigen sich besonders auf der gesellschaftlichen Makro-Ebene die ,groben Grenzziehungen“ in große Personenblöcke, die nach religiöser Anschauung oder Migrationshintergrund vorgenommen werden. Soziale Vielfalt wird an dieser Stelle zumeist als stark zu reflektierende Herausforderung der Zukunft gesehen, die angegangen und moderiert werden muss. Andernfalls wird der gesellschaftliche Zusammenhalt in Gefahr gesehen. Hier zeigen sich diverse Herstellungsweisen von Differenz in der Praxis: Neben der diskursiven Hervorbringung von Differenz durch die Benennung von Bevölkerungsblöcken einer konfessionellen Zugehörigkeit (Othering), einer eigenen oder generationenübergreifenden Migrationserfahrung allgemein (Postmigration, Hybridität), oder der Vermischung von gesellschaftlichen Problemlagen und sozialen Gruppen (boundary making: symbolic \& social), kommen in diesem Zusammenhang individuelle Lebensverläufe und -situationen abhanden.

Letztlich haben die Daten gezeigt, wie vielschichtig die Wirkfelder operativer, gesellschaftlich agierender Stiftungen sein können und in welchem Rahmen sie sich bewegen. Somit muss die Frage danach, wie Stiftungen mit sozialer Differenz umgehen, im Spannungsfeld der Argumentationskonstellationen zu sozialen Differenzkategorien einerseits sowie der Beziehungsherstellung zu Zielgruppen und staatlichen Akteuren andererseits beantwortet werden. Sodann lässt sich auch eine Aussage darüber treffen, welche Bedeutung Stiftungen als zivilgesellschaftliche Akteure zugesprochen werden kann.

Werden Stiftungen allgemein als Dritter-Sektor-Organisationen heuristisch in einem Raum zwischen Markt, Staat und Gemeinschaft vermutet, lässt sich diese Einordnung etwas präzisieren. Agieren Stiftungen als soziale (Wohlfahrts-) Organisation in die Mikro-Ebene hinein, indem sie Individuen unterstützen, treten sie als Partnerin des Staates auf: die staatlicherseits fehlenden Fürsorgeleistungen werden ergänzt, Bedarfe durch den lokalen Fokus erkannt und differenziert aufgearbeitet. Die Handlungsziele wirken dann ideell. Sie bewegen sich diesem Bereich in keiner rechtlichen Grauzone: als private Organisationen dürfen sie ergänzende, diversen Mindeststandards entsprechende Angebote zur Weiterbildung, für Dienstleistungen oder zur Mittelvergabe machen, solange sie staatliche Strukturen nicht in Frage stellen. An dieser Stelle ist das Spannungsverhältnis ausgeglichen.

Auf der Meso-Ebene imitieren Stiftungen die Handlungslogik von privaten Unternehmen. Gewinnmaximierung, Kommunikationsarbeit sowie Eigendarstellungen stehen hier im Vordergrund der Handlungsziele. Da sie als private Organisationen gelten, ist dies nicht verwerflich und möglicherweise sogar zur eigenen Profilierung unumgänglich.

Als zivilgesellschaftliche Gegenspielerinnen gegenüber dem Staatswesen setzen sich operative Stiftungen auf der Makro-Ebene ein. An dieser Stelle findet sich das vielfach diskutierte „Demokratiedefizit“: Bis zum welchem Grad 
dürfen Stiftungen als zivile Organisationen gesellschaftliche Verhältnisse beeinflussen? Inwiefern reproduzieren sie staatliche Herrschaft, was nicht zu ihren Aufgaben zählt? Unproblematisch erscheint in diesem Kontext die bloße Kritik an staatlichen Maßnahmen, Interventionen oder Vorgaben. Letztlich steht diese Option allen Bürgerinnen und Bürgern aufgrund rechtsstaatlicher Standards offen. Wenn allerdings in politische Prozesse eingegriffen werden kann, ob beschleunigend oder konterkarierend, was aufgrund beispielsweise personeller Überschneidungen vorkommen kann, entsteht eine diffuse Gemengelage. Diesen Punkt präziser zu bewerten, ist aufgrund der vorliegenden Daten hier nicht möglich und müsste namentlich mit Hilfe einer Netzwerkanalyse weitergeführt werden.

Mit dem starken Fokus auf die Kommunikationsarbeit entziehen sich insbesondere politische Stiftungen dem Vorwurf des Demokratiedefizits, da sie nicht aktiv in staatliches Handeln hineinwirken. Eine andere Lösung zum Demokratiedefizit ist es, dass sich das Stiftungswesen durch die Zunahme an Gründungen als „Massenphänomen“ geriert und gleichzeitig die Bürgerbeteiligung priorisierend in der Stiftungsarbeit behandelt. Sie stärkt dadurch Bürgerschaft auf legitime Art und Weise. Daher ist das Handlungsziel der Bürgerbeteiligung sehr zentral. Zudem zeigen sich in Bezug zu bisherigen Untersuchungen, dass Überlegungen und Analysen zur regionalen Einbettung zu Stiftungen gänzlich fehlen.

Allerdings können Stiftungen gerade diese Vielschichtigkeit als Organisationstyp gleichzeitig als Antrieb für soziale Innovationen nutzen: Laut BlättelMink/Menez zeichnet es soziale Innovationen aus, dass sie ,widersprüchliche und gegensätzliche Anforderungen, die sich aus dynamischen Umwelten ergeben, anzupassen“ (2015: 203) im Stande sind. Stiftungen verstehen Innovationen als Privileg, internationale Maßnahmen ohne großen Aufwand, den politische Konsensfindungsprozesse mit sich bringen, in den deutschen Kontext adaptieren zu können oder neue Fördermaßnahmen für benachteiligte Zielgruppen zu entwickeln. Inwieweit sie auf den verschiedenen Wirkebenen, gerade auf der gesellschaftlichen Ebene, Innovationspotenzial zeigen, ist ebenfalls ungeklärt geblieben. Denn dass Stiftungen nicht mehrheitsfähige Meinungen unterstützen oder zumindest bis dahin fehlende Gesellschaftsbilder entwerfen, kann an dieser Stelle für die überwiegende Anzahl und aufgrund der vorliegenden Ergebnisse nicht bejaht werden. Vielmehr zeichnet sich ein Bild ab, in dem eine Elite für eine Nachwuchselite eintritt und gleichzeitig für Bedürftige sorgt. Die vorhanden geglaubten „Randgruppen“ in Hinblick auf Ethnizität, Religion und Migration werden als gesellschaftliche Narrative weitestgehend reproduziert. Im Bereich der Fürsorge und Unterstützungsleistungen sozialer Gruppen dominieren sozio-ökonomische, mit individuellen Lebenslagen versehene Argumente. 
Aus der Gesamtbetrachtung ergeben sich bezogen auf die Argumentationskonstellationen zu sozialer Differenz folgende Anhaltspunkte: Die Studienergebnisse zeigen, dass Stiftungen auf der Mikro-Ebene bei der Begründung ihrer Projektziele die Leitdifferenz „Klasse“ verwenden. Wenn es um die Förderung von Individuen oder Familien geht, findet gehäuft ein ,doing class while undoing other differences" statt. Die Argumentationen wurden hier individualistischer, die Äußerungen differenzierter. Differenzmerkmale wurden gegeneinander relativiert, einige wurden negiert. Die Interviewten wogen die Argumente stärker ab. Wenn auf der Meso-Ebene das Ziel eines Vorhabens in eine Kommunikationsstrategie eingebettet war, die nach außen einen Paradigmenwechsel signalisieren sollte, kamen gehäuft Redeweisen des Diversity Managements zum Einsatz. Klassismen rückten in den Hintergrund, es sollten Potenziale und Erfolge aufgezeigt werden. Auf der Makro-Ebene der Argumentationen wurden die Differenzgruppen gröber gezogen: Hier finden sich häufiger Prozesse des Otherings oder des Boundary makings, die die Bevölkerung in größere Menschenblöcke zusammenfassen und individuelle Lebenslagen außer Acht lassen. Diese Argumentationen kamen besonders dann zum Einsatz, wenn die Stiftungsvertretenden, ganz gleich welcher Hierarchieebene, politische Erklärungsversuche für soziale Phänomene anführten.

\subsection{Ausblick: Stiftungen als Akteure der öffentlichen Sphäre}

Differenzbenennung findet in öffentlichen Debatten, in Alltagsgesprächen von Menschen und in weiteren unterschiedlichen Situationen permanent statt. Die vorliegende Studie sollte einen Einblick in einen Bereich geben, in der dies im Kontext zivilgesellschaftlichen Handelns geschieht. Inwiefern diese Differenzbenennungsmuster in anderen Sphären vermutet werden können, muss in weiterführenden Studien untersucht werden. Bereits in der Studie „Habituelle Konstruktion sozialer Differenz“ (2008) kam Kubisch zum Schluss, dass zum Beispiel räumlichen Binnendifferenzen wie Ost-/Westdeutschland in der alltäglichen Arbeit von Wohlfahrtverbänden eine größere Rolle zu kommt als die prominent diskutierten ethnischen Unterschiede.

Stiftungen sind alte Organisationsformen, doch es muss einen Grund geben, aus dem sie sich über Jahrtausende, in der Form nachweisbar seit der Antike, im Einzelbeispiel seit dem Mittelalter, bewähren konnten. Vielleicht ist es ihre Anpassungsfähigkeit an bestehende gesellschaftliche Zustände, die sie dazu veranlasst haben, so vielschichtig zu sein, dass sie, bildlich gesprochen, Eigenschaften des gehobenen Golfclubs, des Interessen vertretenden ADAC-Verbands und der gemeinwohlorientierten Caritas in sich vereinen können. 
Festzuhalten ist, dass die Argumentationsmuster im Bereich der individuellen Förderung als vergleichsweise ausdifferenziert und die sozioökonomische Lage priorisierend bezeichnet werden kann. Dagegen treten im Bereich der gesellschaftspolitischen Wirkungsfelder, auf denen es darum geht, Gesellschaft zu erklären und Zusammenhalt zu stärken, Kollektivismen zu Tage, vermittels derer Differenz stärker angelehnt an öffentliche Diskussionen reproduziert wird. Mit Blick auf prominente Neugründungen und deren parteiliche Anbindungen dürfte sich dieser Vorgang verschärfen. Die Analyse der jeweiligen Funktionsweise von Differenzbenennung im Auge zu behalten, ist daher wünschenswert, um soziale Strukturen zu ergründen und anschließend die Konsequenzen abschätzen zu können (vgl. Ibn Kladun 1377 [2011]: 405).

Durch die Sozialwissenschaft wird den Stiftungen, wenn nicht selbst erklärt, die Funktion auferlegt, unabhängig von tagespolitischen Konjunkturen, gesellschaftlicher Innovationsmotor und Anschieberinnen nicht-mehrheitsfähiger Meinungen zu sein. Möchten sie diese erfüllen, müssen sie sich klarer absetzen und eigene Impulse erzeugen. Vielleicht ist der für Juni 2019 mehrtägig angesetzte große StiftungsTag in diesem Sinne zu verstehen. Er lautet „Unsere Demokratie“. In zahlreichen Workshops wird auch zum Umgang durch Gefahren des Rechtspopulismus, Hate Speech und digitaler Debattenkultur $^{26}$ diskutiert. Prantl's eingangs zitierte Aussage über die starke deutsche Demokratie, die allein vor allem im Bereich des Minderheitenschutzes zu stärken sei, wirkt im Rückblick nicht überzeugend ${ }^{27}$. An diesem Punkt sind die intersektionalen Analysedimensionen von Klassismen, Ethnozentrismen und Genderismen des „türkischen Gemüsehändlers“ noch unangetastet.

26 Diese werden in den letzten Jahren zunehmend wissenschaftlich eruiert. (Beispiele: Scharloth 2017; Assimakopoulos, Stavros u.a. 2017; Eickelmann 2017; Butler 2018; Sponholz 2018).

27 Ähnlich in der Gegenthese im Tagesspiegel von Kamuran Sezer: https://www.tagesspiegel.de/meinung/gastbeitrag-die-deutsche-demokratie-ist-schwach/7507468.html, Abruf: April 2019 


\section{Quellenverzeichnis}

Abels, Gabriele/Behrens, Maria (1998): ExpertInneninterviews in der Politikwissenschaft. Das Beispiel Biotechnologie. In: Österreichische Zeitschrift für Politikwissenschaft 27/1: 79-92.

Abbott, Andrew (1988): The System of Professions: An Essay on the Division of Expert Labor. Chicago.

Adloff, Frank (2005): Zivilgesellschaft. Theorie und politische Praxis. Frankfurt/New York.

Frank Adloff (2004): Wozu sind Stiftungen gut? Zur gesellschaftlichen Einbettung des deutschen Stiftungswesens. In: Leviathan. Zeitschrift für Sozialwissenschaft, 32/2: 269-285.

Adloff, Frank (2002): Förderstiftungen: eine Untersuchung zu den Destinatären und Entscheidungsprozessen. Arbeitspapier. Opusculum Nr. 9.

Akkaya, Gülcan (2012): Nichtregierungsorganisationen als Akteure der Zivilgesellschaft. Wiesbaden.

Altendorf, Hans (1978): Arbeiterkinder an den Hochschulen: soziale Selektion, materielle Lage, Ausbildungsförderung. Köln.

Amirpur, Donja (2016): Migrationsbedingt behindert? Familien im Hilfesystem. Eine intersektionale Perspektive, Bielefeld.

Anheier, Helmut K. (2001): Foundations in Europe: A comparative Perspective. In: Schlüter, Andreas u.a. (Hg.): Foundations in Europe. London.

Anheier, Helmut K. (2003): Das Stiftungswesen in Zahlen. Eine sozial-ökonomische Strukturbeschreibung deutscher Stiftungen. In: Bertelsmann Stiftung: Handbuch Stiftungen. Wiesbaden: 47-82.

Anheier, Helmut K./Förster, Sarah/Mangold, Janina/Striebing, Clemens (2017a): Stiftungen in Deutschland 1. Eine Verortung. Wiesbaden.

Anheier, Helmut K./Förster, Sarah/Mangold, Janina/ Striebing, Clemens (Hg.) (2017b): Stiftungen in Deutschland 2. Wirkungsfelder. Wiesbaden.

Anheier, Helmut K./Förster, Sarah/Mangold, Janina/Striebing, Clemens (Hg.) (2017c): Stiftungen in Deutschland 3. Portraits und Themen. Wiesbaden.

Anheier, Helmut K./Priller, Eckhard/Zimmer, Annette (2000): Zur zivilgesellschaftlichen Dimension des Dritten Sektors. In: Klingemann, Hans-Dieter/Neidhardt, Friedhelm (Hg.): Die Zukunft der Demokratie. Herausforderungen im Zeitalter der Globalisierung. WZB-Jahrbuch. Wissenschaftszentrum Berlin für Sozialforschung. Berlin: 71-98.

Anthias, Floya (2005): Social Stratification and Social Inequality: Models of Intersectionality and Identity. In: Devine, Fiona u.a. (Hg.): Rethinking Class, Culture, Identities and Lifestyles. London: 24-45.

Assimakopoulos, Stavros/Baider, Fabienne H./Millar, Sharon (2017): Online Hate Speech in the European Union: A Discourse-Analytic Perspective. Wiesbaden.

Aulenbacher, Brigitte/Meuser, Michael/Riegraf, Birgit (2010): Soziologische Geschlechterforschung: Eine Einführung. Wiesbaden.

Barlösius, Eva (2004): Kämpfe um soziale Ungleichheit. Machttheoretische Perspektiven. Wiesbaden. 
Becker, Elke/Wolf, André Christian (1999): Stiften findet Stadt. Eine Arbeitshilfe zum Aufbau von Bürgerstiftungen. Stiftung Mitarbeit (Hg.). Bonn.

Bednarz-Braun, Iris/Heß-Meining, Ulrike (2004): Migration, Ethnie und Geschlecht. Theorieansätze - Forschungsstand - Forschungsperspektiven. Wiesbaden.

Berghaus, Margot (2003): Luhmann leicht gemacht. Köln.

Berger, Peter A./Schmidt, Volker H. (Hg.) (2004): Welche Gleichheit, welche Ungleichheit? Grundlagen der Ungleichheitsforschung. Wiesbaden.

Berger, L. Peter/Luckmann, Thomas (1997): Die gesellschaftliche Konstruktion der Wirklichkeit. Eine Theorie der Wissenssoziologie. Frankfurt am Main.

Benz, Arthur (2001): Der moderne Staat. Grundlagen der politologischen Analyse. München.

Bhabha, Homi K. (1994): The location of culture. London.

Birkhölzer, Karl (2005): Drittes System und Soziale Ökonomie im europäischen Kontext. In: Birkhölzer, Karl/Klein, Ansgar/Zimmer, Annette/Priller, Eckhard (Hg.): Dritter Sektor/Drittes System. Theorie, Funktionswandel und zivilgesellschaftliche Perspektiven. Wiesbaden: 71-92.

Birkhölzer, Karl/ Priller, Eckhard/Zimmer, Annette (2005): Dritter Sektor/Drittes System im Spiegel von Theorie und Praxis - eine Einführung. In: Birkhölzer, Karl/ Klein, Ansgar/Zimmer, Annette/Priller, Eckhard (Hg.): Dritter Sektor/Drittes System. Theorie, Funktionswandel und zivilgesellschaftliche Perspektiven. Wiesbaden: 41-47.

Blättel-Mink, Birgit/Menez, Raphael (2015): Innovation und Organisation. In: BlättelMink, Birgit/Menez, Raphael (Hg.): Kompendium der Innovationsforschung. Wiesbaden: 199-208.

Böckenförde, Ernst Wilhelm (1976): Die Bedeutung der Unterscheidung von Staat und Gesellschaft im demokratischen Sozialstaat der Gegenwart. In: Böckenförde, Ernst Wilhelm (Hg.): Staat - Gesellschaft - Freiheit. Frankfurt a.M.

Bogner, Alexander/Menz, Wolfgang (2009a): Experteninterviews in der qualitativen Sozialforschung. Zur Einführung in eine sich intensivierende Methodendebatte. In: Bogner, Alexander/Littig, Beate/Menz, Wolfgang (Hg.): Experteninterviews. Theorien, Methoden, Anwendungsfelder. Wiesbaden: 7-34.

Bogner, Alexander/Menz, Wolfgang (2009b): Das theoriegenerierende Experteninterview. Erkenntnisinteresse, Wissensformen, Interaktion. In: Bogner, Alexander/ Littig, Beate/Menz, Wolfgang (Hg.): Experteninterviews. Theorien, Methoden, Anwendungsfelder. Wiesbaden: 61-98.

Bogner, Alexander/Menz, Wolfgang (2001): Deutungswissen und Interaktion. Zu Methodologie und Methodik des theoriegenerierenden Experteninterviews. In: Soziale Welt, 52/4: 477-500.

Bohnsack, Ralf/Marotzki, Winfried/Meuser, Michael (2006): Hauptbegriffe qualitativer Sozialforschung. Opladen.

Bommes, Michael u.a. (Hg.) (2008): Migranten am START. Bildungskarrieren von begabten Zuwandererkindern. Eine Studie im Auftrag der START-Stiftung. Osnabrück.

Borgolte, Michael (2004): Die mittelalterliche Kirche. In: Enzyklopädie deutscher Geschichte, 17. München.

Bourdieu, Pierre (1983): Ökonomisches Kapital, kulturelles Kapital, soziales Kapital. In: Kreckel, Reinhard (Hg.): Soziale Ungleichheiten. Soziale Welt Sonderband 2. Göttingen: 183-198. 
Bös, Mathias (2008): Ethnizität. In: Baur, Nina/ Korte, Hermann/ Löw, Martina/ Schroer, Markus (Hg.): Handbuch Soziologie: 55-76.

Brubaker, Rogers (2015): Grounds for Difference. Cambridge.

Brubaker, Rogers (2016): trans. Gender and Race in an Age of Unsettled Identities. Princeton.

Bukow, Wolf-Dietrich/ Heck, Gerda/ Schulze, Erika/ Y1ldı, Erol (Hg.) (2011): Neue Vielfalt in der Urbanen Stadtgesellschaft. Wiesbaden.

Bundesverband Deutscher Stiftungen. www.stiftungen.org (Stand: Febr. 2018)

"Verzeichnis Deutscher Stiftungen" des Bundesverbands Deutscher Stiftungen. Die "StiftungsUmfrage" erfasst und porträtiert 16.000 Stiftungen aus dem Bundesgebiet zu den Themen, Budget, Budgetverteilung, Ziele, Motivationen usw. (Stand: Sept. 2012)

Bundesverband Deutscher Stiftungen e.V. (2013a): Bürgerstiftungen 2030. Strategien für die Zukunft. Analysen, Daten, Trends 2013/14. Berlin.

Bundesverband Deutscher Stiftungen e.V. (2013b): Kommunale Stiftungen in Deutschland. Bestandsaufnahme, Chancen und Herausforderungen. Berlin.

Bundesverband Deutscher Stiftungen e.V. (2011): Verzeichnis Deutscher Stiftungen. Zahlen, Daten, Fakten. Berlin.

Bürgerliches Gesetzbuch in der Fassung der Bekanntmachung vom 2. Januar 2002 (BGBl. I S. 42, 2909; 2003 I S. 738), das zuletzt durch Artikel 2 des Gesetzes vom 21. Februar 2017 (BGB1. I S. 258) geändert worden ist. Bei: Bundesministerium der Justiz und für Verbraucherschutz. URL: https://www.gesetze-im-internet.de/bundesrecht/bgb/gesamt.pdf (abgerufen 10. März 2017)

Butler, Judith (2018): Haß spricht - zur Politik des Performativen. 6. Auflage. Berlin.

Butler, Judith (1991): Das Unbehagen der Geschlechter. Baden-Baden.

Campenhausen, Axel (2015): Stiftungen. In: Religion in Geschichte und Gegenwart 2015. Wörterbuch.

Canan, Coşkun (2015): Identitätsstatus von Einheimischen mit Migrationshintergrund. Wiesbaden.

Casale, Rita/ Koller, Hans-Christoph / Ricken, Norbert (Hg.) (2014): Heterogenität: zur Konjunktur eines pädagogischen Konzepts. Paderborn.

Castro Varela, Marìa do Mar/ Dhawan, Nikita (2005): Postkoloniale Theorie. Eine kritische Einführung. Bielefeld.

Çetin, Zülfukar (2012): Homophobie und Islamophobie. Intersektionale Diskriminierungen am Beispiel binationaler schwuler Paare in Berlin. Bielefeld.

Çetin, Zülfukar (2013): Kritik an der Ausblendung der Dominanzverhältnisse: Intersektionalität als Herausforderung in der weißen Sozialwissenschaft. In: Erwägen, Wissen, Ethik: Forum für Erwägungskultur, 24/ 3: 369-371.

Collin, Finn (2008): Konstruktivismus für Einsteiger. Paderborn.

Cox, Taylor (1994): Cultural Diversity in organizations. Theory, research, practice, San Francisco.

Crenshaw, Kimberlé Williams (1988): Race, Reform, and Retrenchment: Transformation and Legitimation in Antidiscrimination Law. In: Harvard Law Review 101/ 7: 1331-1387.

Dağlar-Sezer, Nilgün (2013): Bürgerschaftliches Engagement und soziale Vielfalt. In: Sozialwissenschaft und Berufspraxis, 1/2013: 145-156. 
Dağlar, Nilgün (2008): Diversity Management: Eine Personalstrategie im medialen Diskurs der Akteure. Unveröffentlichte Magisterarbeit, Westfälische WilhelmsUniversität Münster, Institut für Soziologie. Münster.

Desiderius-Erasmus-Stiftung: www.erasmus-stiftung.de (Stand: August 2018)

Dippelhofer-Stiem, Barbara (2017): Sind Arbeiterkinder im Studium benachteiligt? Empirische Erkundungen zur schichtspezifischen Sozialisation an der Universität. Weinheim.

Eickelmann, Jennifer (2017): "Hate Speech" und Verletzbarkeit im digitalen Zeitalter: Phänomene mediatisierter Missachtung aus Perspektive der Gender Media Studies. Bielefeld.

Eickelpasch, Rolf (2001): Hierarchie und Differenz. Anmerkungen und Anfragen zur "konstruktivistischen Wende" in der Analyse sozialer Ungleichheit. In: Rademacher, Claudia/ Wiechens, Peter (Hg.): Geschlecht - Ethnizität - Klasse. Zur sozialen Konstruktion von Hierarchie und Differenz. Opladen: 53-64.

Elias, Norbert (1969): Die höfische Gesellschaft. Berlin.

Etzioni, Amitai (1973): The Third Sector and Domestic Missions. In: Public Administration Review, 33/4: 314-323.

Foroutan, Naika (2018): Die postmigrantische Perspektive. Aushandlungsprozesse in pluralen Gesellschaften. In: Hill, Marc/Yıldız, Erol (Hg.): Postmigrantische Visionen. Erfahrungen - Ideen - Reflexionen. Bielefeld: 15-28.

Foroutan, Naika (2016): Postmigrantische Gesellschaften. In: Brinkmann, Heinz Ulrich/ Sauer, Martina (Hg.): Einwanderungsgesellschaft Deutschland. Entwicklung und Stand der Integration. Wiesbaden: 227-254.

Foucault, Michel (1998): Analytik der Macht. Frankfurt am Main.

Foucault, Michel (1978): Über Sexualität, Wissen und Wahrheit. Berlin.

Friedrich-Ebert-Stiftung: www.fes.de (Stand: Febr. 2018)

Friedrich-Naumann-Stiftung für die Freiheit: www.freiheit.org (Stand: Febr. 2018)

Garfinkel, Harold (1967): Studies in Ethnomethodology. Englewood Cliffs.

Gildemeister, Regine/Hericks, Katja (2012): Geschlechtersoziologie: Theoretische Zugänge zu einer vertrackten Kategorie des Sozialen. München.

Gildemeister, Regine/ Wetterer, Angelika (1992): Wie Geschlechter gemacht werden. Die soziale Konstruktion der Zweigeschlechtlichkeit und ihre Reifizierung in der Frauenforschung. In: Knapp, Gudrun-Axeli/Wetterer, Angelika (Hg.): TraditionenBrüche. Entwicklungen feministischer Theorie. Freiburg im Breisgau.

Gläser, Jochen/ Laudel, Grit (2010): Experteninterviews und qualitative Inhaltsanalyse als Instrumente rekonstruierender Untersuchungen. Wiesbaden.

Goffman, Erving (1994): Interaktion und Geschlecht. Frankfurt am Main.

Gordon, Raymond L. (1975): Interviewing: strategy, techniques, and tactics. Homewood, Illinois.

Graf, Peter (2006): Religionen in Migration: Grenzüberschreitung als Aufforderung zum Dialog. Göttingen.

Gümen, Sedef (2001): Das Soziale des Geschlechts. Frauenforschung und die Kategorie „Ethnizität“. In: Hark, Sabine (Hg.): Dis/Kontinuitäten: Feministische Theorie. Opladen: 136-154.

Hahn, Olaf (2009): Stiftungen und die Förderung der Integration von Migranten. In: Rat für Migration: Politische Essays zu Migration und Integration. 1/2009. Osnabrück. 
Hall, Stuart (2004): Wann gab es ,das Postkoloniale“? Denken an der Grenze. In: Sebastian Conrad (Hg.): Jenseits des Eurozentrismus. Postkoloniale Perspektiven in der Geschichts- und Kulturwissenschaft. Frankfurt a.M.: 219-246.

Hall, Stuart (1999) Ethnizität: Identität und Differenz. In: Jan Engelmann (Hg.): Die kleinen Unterschiede. Der Cultural Studies-Reader. Frankfurt a.M.: 83-98.

Hans-Böckler-Stiftung: www.boeckler.de (Stand: Febr. 2018)

Hanns-Seidel-Stiftung: www.hss.de (Stand: Febr. 2018)

Hegel, Georg Wilhelm Friedrich (1821 [1995]): Grundlinien der Philosophie des Rechts: Naturrecht und Staatswissenschaft im Grundrisse. Berlin.

Heinrich Böll Stiftung: www.boell.de (Stand: Febr. 2018)

Heisterkamp, Ulrich (2014): Think Tanks der Parteien? Eine vergleichende Analyse der deutschen politischen Stiftungen. Wiesbaden.

Hetfleisch, Gerhard (2013): Migrationsforschung als Apologie herrschender Verhältnisse am Beispiel Hartmut Essers. In: Mecheril, Paul; Arens, Susanne; Melter, Claus; Romaner, Elisabeth \& Thomas-Olalde, Oscar (2013a) (Hg.): Migrationsforschung als Kritik?: Spielräume kritischer Migrationsforschung. Wiesbaden: 213-230.

Hill, Marc/ Y1ldız, Erol (2018): Postmigrantische Visionen. Erfahrungen - Ideen - Reflexionen. Bielefeld.

Hill, Stephan (1973): Supervisory Roles and the Man in the Middle: Dock Foremen. In: The British Journal of Sociology, 24/2: 205-221.

Hiller, Gotthilf Gerhard (2012): Heterogenität: Ärgernis und Chance für die Schule. In: Sonderpädagogische Förderung heute 3: 252233

Hirschauer, Stefan (Hg.) (2017): Un/doing Differences. Praktiken der Humandifferenzierung. Weilerswist.

Hirschauer, Stefan (2017): Humandifferenzierung. Modi und Grade sozialer Zugehörigkeit. In: Hirschauer, Stefan (Hg.) (2017): Un/doing Differences. Praktiken der Humandifferenzierung. Weilerswist: 29-54.

Hirschauer, Stefan (2014): Un/doing Differences. Die Kontingenz sozialer Zugehörigkeiten. In: Zeitschrift für Soziologie, 43/3: 170-191.

Hirschauer, Stefan (2011): Konstruktivismus. In: Bohnsack, Ralf/ Marotzki, Winfried/ Meuser/ Michael (Hg.): Hauptbegriffe qualitativer Sozialforschung. Opladen \& Fermington Hills: 102-104.

Hitzler, Ronald (1986) Die Attitüde der künstlichen Dummheit. Zum Verhältnis von Soziologie und Alltag. In: Sozialwissenschaftliche Informationen (SOWI), 15/ 3: 53-59.

Howaldt, Jürgen/ Schwarz, Michael (2010): "Soziale Innovation" im Fokus: Skizze eines gesellschaftstheoretisch inspirierten Forschungskonzepts. Bielefeld.

Ibn Khaldūn (1377 [2011]): Die Muqaddima. Betrachtungen zur Weltgeschichte. Aus dem Arabischen von Alma Giese. München.

Kaufmann, Franz-Xaver (1998): Globalisierung und Gesellschaft. In: APuZ, B 18/98: 3-10.

Kaiser, Robert (2014): Qualitative Experteninterviews: Konzeptionelle Grundlagen und praktische Durchführung. Wiesbaden.

Kaloianov, Radostin (2013): Exzellenz oder Existenz? Die Kritikposition von MigrantInnen im Feld gegenwärtiger kritischer Gesellschaftsforschung. In: Mecheril, Paul; Arens, Susanne; Melter, Claus; Romaner, Elisabeth \& Thomas-Olalde, Oscar 
(2013a) (Hg.): Migrationsforschung als Kritik?: Spielräume kritischer Migrationsforschung. Wiesbaden: 109-125.

Kazzazi, Kerstin/ Treiber, Angela/ Wätzhold, Tim (Hg.) (2016): Migration, Religion, Identität: Aspekte transkultureller Prozesse. Wiesbaden.

Keane, John (2003): Global Civil Society? Cambridge. URL: https://is.muni.cz/el/1423/jaro2007/SOC711/2742958/Keane_GCS.pdf (abgerufen 10.03.2017)

Keane, John (1998): Civil Society. Old Images, New Visions. Oxford.

Kerchner, Brigitte (2011): Diskursanalyse der Intersektionalität. In: Smykalla, Sandra/ Vinz, Dagmar (Hg.): Intersektionalität zwischen Gender und Diversity. Theorien, Methoden und Politiken der Chancengleichheit. Münster: 144-160.

Kinne, Peter (2015): Diversity 4.0. Zukunftsfähig durch intelligent genutzte Vielfalt. Wiesbaden.

Klaassen, Holger (2016): Die Finanzierung parteinaher Stiftungen in den Ländern. Zugleich eine Diskussion des Begriffs der "parteinahen Stiftung". Marburg.

Klinger, Cornelia (2008): Überkreuzende Identitäten - Ineinandergreifende Strukturen. Plädoyer für einen Kurswechsel in der Intersektionalitätsdebatte. In: ÜberKreuzungen. Fremdheit, Ungleichheit, Differenz. Münster: 38-67.

Klinger, Cornelia/ Knapp, Gudrun-Axeli (Hg.) (2008): ÜberKreuzungen. Fremdheit, Ungleichheit, Differenz. Münster.

Klinger, Cornelia (2003): Ungleichheit in den Verhältnissen von Klasse, Rasse und Geschlecht. In: Knapp, Gudrun-Axeli/ Wetterer, Angelika (Hg.): Achsen der Differenz. Gesellschaftstheorie und feministische Kritik II. Münster: 14-48.

Klinger, Cornelia/ Knapp, Gudrun-Axeli/ Sauer, Birgit (2007): Achsen der Ungleichheit. Zum Verhältnis von Klasse, Geschlecht und Ethnizität. Frankfurt a.M./ New York

Knapp, Gudrun-Axeli (2013): Zur Bestimmung und Abgrenzung von "Intersektionalität". Überlegungen zu Interferenzen von "Geschlecht", "Klasse" und anderen Kategorien sozialer Teilung. In: Erwägen - Wissen - Ethik 24/3: 341-357.

Knapp, Gudrun-Axeli (2008): Verhältnisbestimmungen: Geschlecht, Klasse, Ethnizität in gesellschaftstheoretischer Perspektive. In: Klinger, Cornelia/ Knapp, GudrunAxeli (Hg.): ÜberKreuzungen. Fremdheit, Ungleichheit, Differenz. Münster: 138170.

Knapp, Gudrun-Axeli (2005): „Intersectionality“ - ein neues Paradigma feministischer Theorie? Zur transatlantischen Reise von „Race, Class, Gender“. In: Feministische Studien 23: 68-81.

Knapp, Gudrun-Axeli/ Wetterer, Angelika (Hg.) (2003): Achsen der Differenz. Gesellschaftstheorie und feministische Kritik II. Münster.

Knorr-Cetina, Karin (1989): Spielarten des Konstruktivismus. Einige Notizen und Anmerkungen. In: Soziale Welt, 40/1: 86-96.

Koall, Iris/ Bruchhagen, Verena/ Höher, Friederike (Hg.) (2007): Diversity Outlooks. Managing Diversity zwischen Ethik, Profit und Antidiskriminierung. Hamburg.

Kocka, Jürgen (2001): Zivilgesellschaft. Zum Konzept und seiner sozialgeschichtlichen Verwendung. In: Kocka, Jürgen/ Nolte, Paul/ Randeria, Shalini/ Reichardt, Sven: Neues über Zivilgesellschaft. Aus historisch-sozialwissenschaftlichem Blickwinkel. Berlin. 
Kocka, Jürgen (2002): Das Bürgertum als Träger der Zivilgesellschaft - Traditionslinien, Entwicklungen, Perspektiven. In: Enquete-Kommission "Zukunft des Bürgerschaftlichen Engagements" (Hg.): Bürgerschaftliches Engagement und Zivilgesellschaft. Opladen: 15-22.

Konrad-Adenauer-Stiftung: www.kas.de (Stand: Febr. 2018)

Kubisch, Sonja (2008): Habituelle Konstruktion sozialer Differenz. Eine rekonstruktive Studie am Beispiel von Organisationen der freien Wohlfahrtspflege. Wiesbaden.

Krimmer, Holger/ Weitemeyer, Birgit/ Kleinpeter, Saskia/ Vogt, Benedikt/ Schönfeld, Friedrich von (2014): Transparenz im Dritten Sektor. Eine wissenschaftliche Bestandsaufnahme. Hamburg.

Lamont, Michèle/ Molnár, Virág (2002): The study of boundaries in the social sciences. In: Annual Review of Sociology, 28: 167-195.

Langsdorff, Nicole von (2012): Mädchen auf ihrem Weg in die Jugendhilfe. Intersektionale Wirkprozesse im Lebensverlauf. Opladen, Berlin, Toronto.

Lenz, Ilse (1996): Grenzziehungen und Öffnungen: Zum Verhältnis von Geschlecht und Ethnizität zu Zeiten der Globalisierung. In: Lenz, Ilse; Germer, Andrea; Hasenjürgen, Brigitte (Hg.): Wechselnde Blicke. Frauenforschung in internationaler Perspektive. Opladen: 200-229.

Littig, Beate (2009): Interviews mit Experten und Expertinnen. Überlegungen aus geschlechtertheoretischer Sicht. In: Bogner, Alexander (Hg.): Das Experteninterview. Theorie, Methode, Anwendung. Wiesbaden: 181-198.

Machold, Claudia (2015): Kinder und Differenz. Eine ethnografische Studie im elementarpädagogischen Kontext. Wiesbaden.

Mannheim, Karl: Beiträge zur Theorie der Weltanschauungs-Interpretation. In: Mannheim, Karl: Wissenssoziologie. Luchterhand, Neuwied 1964 [1921-22]: 91-154.

Marx Ferree, Myra (2010): Die diskursiven Politiken feministischer Intersektionalität. In: Lutz, Helma/ Herrera Vivar, María Teresa/ Supik, Linda (Hg.): Fokus Intersektionalität. Bewegungen und Verortungen eines vielschichtigen Konzepts. Wiesbaden: 69-82.

Matoba, Kazuma (2007): Diversity Management: Kommunikativer Prozess zur Entwicklung der “dritten Kultur”. In: Koall, Iris/ Bruchhagen, Verena/ Höher, Friederike (Hg.): Diversity Outlooks. Managing Diversity zwischen Ethik, Profit und Antidiskriminierung. Hamburg: 336-353.

Mead, Margaret (1958): Mann und Weib. Reinbeck.

Mecheril, Paul (2014): Subjektbildung: interdisziplinäre Analysen der Migrationsgesellschaft. Bielefeld.

Mecheril, Paul; Arens, Susanne; Melter, Claus; Romaner, Elisabeth \& Thomas-Olalde, Oscar (2013a) (Hg.): Migrationsforschung als Kritik?: Spielräume kritischer Migrationsforschung. Wiesbaden.

Mecheril, Paul; Arens, Susanne; Melter, Claus; Romaner, Elisabeth \& Thomas-Olalde, Oscar (2013b): Migrationsforschung als Kritik? Eine Annäherung an ein epistemisches Anliegen in 57 Schritten. In Dies. (Hg.): Migrationsforschung als Kritik? (Sowohl in Band I als auch Band II), Wiesbaden: 7-55.

Mecheril, Paul (1999): Wer spricht über wen? Gedanken zu einem (re-)konstruktiven Umgang mit den Anderen in den Sozialwissenschaften. In: Bukow, Wolf-Dietrich/Ottersbach, Markus (Hg.): Der Fundamentalismusverdacht. Plädoyer für eine Neuorientierung der Forschung im Umgang mit allochthonen Jugendlichen, Opladen: 231-266. 
Merten, Heike (1999): Parteinahe Stiftungen im Parteienrecht. Baden-Baden.

Meuser, Michael (2016): Diversität. Staatslexikon 8. Auflage / K1: 789-793.

Meuser, Michael (2013a): Diversity Management - Anerkennung von Vielfalt? In: Pries, Ludger (Hg.): Zusammenhalt durch Vielfalt? Wiesbaden: 167-181.

Meuser, Michael (2013b): Intersektionale Heuristik - Gesellschaftstheorie und rekonstruktive Methodologie. In: Erwägen - Wissen - Ethik 24/3: 431-433.

Meuser, Michael (2011): Ethnomethodologie. In: Bohnsack, Ralf/ Marotzki, Winfried/ Meuser/ Michael (Hg.): Hauptbegriffe qualitativer Sozialforschung. Opladen \& Fermington Hills: 51-55.

Meuser, Michael (2010): Geschlechtersoziologie. In: Kneer, Georg/ Schroer, Markus (Hg.): Handbuch spezielle Soziologien. Wiesbaden: 145-162.

Meuser, Michael/ Nagel, Ulrike (2009): Das Experteninterview - konzeptionelle Grundlagen und methodische Anlage. In: Pickel, Susanne u.a. (Hg.): Methoden der vergleichenden Politik- und Sozialwissenschaft. Wiesbaden: 465 - 479.

Meuser, Michael/ Nagel, Ulrike (2005): Experteninterview und der Wandel der Wissensproduktion. In: Bogner, Alexander (Hg.): Das Experteninterview. Theorie, Methode, Anwendung. Wiesbaden: 33-60.

Meuser, Michael/ Nagel, Ulrike (1994): Expertenwissen und Experteninterview. In: Hitzler, Ronald u.a. (Hg.): Expertenwissen. Opladen: 180-192.

Meuser, Michael/ Nagel, Ulrike (1991): ExpertInneninterviews - vielfach erprobt, wenig bedacht. Ein Beitrag zur qualitativen Methodendiskussion. In: Garz, Detlef/ Kraimer, Klaus (Hg.): Qualitativ-empirische Sozialforschung. Opladen.

Meuser, Michael/ Neusüß, Claudia (Hg.) (2004): Gender Mainstreaming. Konzepte, Handlungsfelder, Instrumente. Bonn: Bundeszentrale für politische Bildung.

Meuser, Michael u.a. (Hg.) (2010): Fragile Sozialität. Inszenierungen, Sinneswelten, Existenzbastler. Ronald Hitzler zum 60. Geburtstag. Wiesbaden.

Müller, Monika (2013): Migration und Religion: Junge hinduistische und muslimische Männer in der Schweiz. Wiesbaden

Pascher, Ute (2001): Die deutschen parteinahen politischen Stiftungen. Hybride Organisationen in der Globalisierung. Berlin.

Plaßmeier, Nike/ Schwippert, Knut/ Bos, Wilfried/ Lankes, Eva-Maria (2004): Heterogenität : eine Herausforderung an die empirische Bildungsforschung. Münster

Paulus, Stefan (2012): Das Geschlechterregime. Eine intersektionale Dispositivanalyse von Work-Life-Balance-Maßnahmen. Bielefeld.

Priller, Eckhard (2002): Zum Stand empirischer Befunde und sozialwissenschaftlicher Theorie und zur Notwendigkeit ihrer Weiterentwicklung. In: Enquete-Kommission "Zukunft des Bürgerschaftlichen Engagements" (Hg.): Bürgerschaftliches Engagement und Zivilgesellschaft. Opladen: 39-54.

Priller, Eckhard/ Zimmer, Annette (2000): Der Dritte Sektor in Deutschland - seine Perspektiven im neuen Millennium. Münsteraner Diskussionspapiere zum Nonprofit-Sektor Nummer 10. Münster.

Pues, Lothar (2010): Praxishandbuch Stiftungen: Stiften auch mit kleinem Vermögen. Stuttgart.

Pues, Lothar (1999): Stiftungen: Vermögen übertragen und verwalten; Praxishandbuch. Stuttgart.

Rademacher, Claudia/ Wiechens, Peter (Hg.) (2001): Geschlecht, Ethnizität, Klasse. Zur sozialen Konstruktion von Hierarchie und Differenz. Opladen. 
Rassem, Mohammed (1960): Die Stiftung als Modell. In: Antaios Nr. 34, 1/1960: 485501.

Reckwitz, Andreas (2015): Drei Versionen des Hybriden: Ethnische, kulturelle und soziale Hybriditäten. In: Kron, Thomas (Hg.): Hybride Sozialität - soziale Hybridität. Weilerswist: 187-200.

Reckwitz, Andreas (2008): Unscharfe Grenzen. Perspektiven der Kultursoziologie. Bielefeld.

Reden, Sitta von (Hg.) (2015): Stiftungen zwischen Politik und Wirtschaft. Geschichte und Gegenwart im Dialog. Oldenburg.

Reinprecht, Christoph/ Latcheva, Rossalina (2016): Migration: Was wir nicht wissen. Perspektiven auf Forschungslücken. Sonderheft 15 der Österreichischen Zeitschrift für Soziologie. Wiesbaden.

Riegel, Christine (2016): Bildung. Intersektionalität. Othering. Pädagogisches Handeln in widersprüchlichen Verhältnissen. Bielefeld.

Rosa-Luxemburg-Stiftung: www.rosalux.de (Stand: Febr. 2018)

Sachverständigenrat Deutscher Stiftungen für Integration und Migration: www.svrmigration.de (Stand: Juli 2018)

Salamon, Lester M./ Anheier, Helmut (1997): Der Nonprofit-Sektor: Ein theoretischer Versuch. In: Anheier, Helmut u.a. (Hg.): Der Dritte Sektor in Deutschland. Organisationen zwischen Staat und Markt im Wandel. Berlin: 211-246.

Salzbrunn, Monika (2012): Gebietskartierung Vielfalt/ Diversity/ Diversité. In: Soziologische Revue 35: 375-394.

Sander, Dominik (2006): Diversity Management. Vielfalt als Ressource. Betriebswirtschaftliche Begründungen. Saarbrücken.

Scharloth, Joachim (Hg.) (2017): Themenheft: Hate speech/Hassrede. Aptum 13/2. Bremen.

Schimank, Uwe (2013): Gesellschaft. Bielefeld.

Schnicke, Falko (2014): Terminologie, Erkenntnisinteresse, Methode und Kategorien Grundfragen intersektionaler Forschung. In: Klein, Christian/ Schnicke, Falko (Hg.): Intersektionalität und Narratologie. Methoden - Konzepte - Analysen. Trier: 1-32.

Schlüter, Andreas (2004): Stiftungsrecht zwischen Privatautonomie und Gemeinwohlbindung. Ein Rechtsvergleich Deutschland, Frankreich, Italien, England, USA. München.

Schnurbein, Georg von (2015): Der Stifter als Unternehmer: Parallelen und Unterschiede der Philanthropie im 19. und 21. Jahrhundert. In: Reden, Sitta von (Hg.): Stiftungen zwischen Politik und Wirtschaft. Geschichte und Gegenwart im Dialog. Oldenburg: 237-260.

Schrader, Kathrin (2013): Drogenprostitution. Eine intersektionale Betrachtung zur Handlungsfähigkeit drogengebrauchender Sexarbeiterinnen. Bielefeld.

Schultz, Ulrike (2011): Intersektionalität, Ethnie und Geschlecht: Umsetzung in der qualitativen Sozialforschung. In: Smykalla, Sandra/ Vinz, Dagmar (Hg.): Intersektionalität zwischen Gender und Diversity. Theorien, Methoden und Politiken der Chancengleichheit. Münster: 128-143.

Schwinn, Thomas (2007): Komplexe Ungleichheitsverhältnisse: Klasse, Ethnie und Geschlecht. In: Klinger, Cornelia/ Knapp, Gudrun-Axeli/ Sauer, Birgit (Hg.): Achsen der Ungleichheit. Zum Verhältnis von Klasse, Geschlecht und Ethnizität. Frankfurt a.M./ New York: 271-286. 
Sepehri, Paivand (2002): Diversity Management in internationalen Organisationen. Wahrnehmung zum Verständnis und ökonomischer Relevanz. München und Mering.

Sezer, Kamuran/ Dağlar, Nilgün (2009): TASD Spektrum II: Die Identifikation der TASD mit Deutschland. Sozialstudie über türkische Akademiker und Studierende. Krefeld/ Dortmund.

Sigmund, Steffen (2004): Solidarität durch intermediäre Institutionen: Stiftungen. In: Beckert, Jens; Eckert, Julia; Kohli, Martin; Streeck, Wolfgang (Hg.): Transnationale Solidarität. Chancen und Grenzen. Frankfurt/New York: 95-108.

Simmel, Georg (1999 [1890]): Über sociale Differenzierung. In: Gesamtausgabe: Aufsätze 1887-1890. Frankfurt a.M.

Soeffner, Hans-Georg (1992): Rekonstruktion statt Konstruktivismus: 25 Jahre "Social construction of reality". In: Soziale Welt , 43/ 4: 476 - 481.

Sökefeld, Martin (2007): Problematische Begriff: "Ethnizität", "Rasse", "Kultur", "Minderheit". In: Schmidt-Lauber, Brigitta (Hg.): Ethnizität und Migration. Einführung in Wissenschaft und Arbeitsfelder. Berlin: 31-50.

Solga, Heike/ Berger, Peter A./ Powell, Justin (2009): Soziale Ungleichheit - Kein Schnee von gestern! Eine Einführung. In: Solga, Heike/ Powell, Justin/ Berger, Peter A. (Hg.): Soziale Ungleichheit. Klassische Texte zur Sozialstrukturanalyse. Frankfurt a.M.: 11-46.

Sprengler, Norman/ Hubrich, David-Karl/ Tamm, Timo/ Alscher, Mareike/ Priller, Eckhard (2011): Landkarte zur Datenlage Dritter Sektor/ Zivilgesellschaft. Projektbericht. Berlin.

Sponholz, Liriam (2018): Hate Speech in den Massenmedien: Theoretische Grundlagen und empirische Umsetzung. Wiesbaden.

Stender, Wolfram (2000): Ethnische Erweckungen. Zum Funktionswandel von Ethnizität in modernen Gesellschaften - ein Literaturbericht. In: Mühlweg 36, 9/4: 6582.

Strachwitz, Rupert Graf (2016): Die Stiftung - ein Paradoxon? Oldenburg.

Strachwitz, Rupert Graf (2015a): Transparente Zivilgesellschaft? Accountability und Compliance in Non-profit-Organisationen. Schwalbach.

Strachwitz, Rupert Graf (2015b): Stiftungen und moderner Staat. Zur Genese und Argumentation einer Legitimitätsdebatte. In: Historische Zeitschrift, Beiheft 66/2015, S. 283-317.

Strachwitz, Rupert Graf (2007): Stiftungen nach der Stunde Null. Die Entwicklung des Stiftungswesens in Westdeutschland nach 1945. In: Geschichte und Gesellschaft 33: 99-126.

Strachwitz, Rupert Graf (Hg.) (1998): Dritter Sektor - Dritte Kraft. Stuttgart.

Striebing, Clemens (2017): Legitimierung von Stiftungen. Bedingungen von Transparenz und Multistakeholder Governance. Wiesbaden.

Stuber, Michael (2002): Die Umsetzung von Diversity in Europa. In: Belinszki, E./ Hansen, K./ Müller, U. (Hg.): Diversity Management. Best practices im internationalen Feld, Münster: 130-154

Then, Volker/ Kehl, Volker (2015): Stiften als soziale Investition an den Grenzen der Sektoren. In: Reden, Sitta von (Hg.): Stiftungen zwischen Politik und Wirtschaft. Geschichte und Gegenwart im Dialog. Oldenburg: 261-282.

Thomas, D. A./ Ely, R. J. (1996): Making differences matter: A new paradigm for managing diversity. In: Harvard Business Review Sept-Oct. 1996: 79-90. 
Tiefensee, Eberhard/ Kraft, Claudia (2011): Religion und Migration: Frömmigkeitsformen und kulturelle Deutungssysteme auf Wanderschaft. Münster

Timmer, Karsten (2005): Stiften in Deutschland. Die Ergebnisse der StifterStudie. Verlag Bertelsmann Stiftung. Gütersloh.

Tuider, Elisabeth (2015): Dem Abwesenden, den Löchern und Rissen empirisch nachgehen: Vorschlag zu einer dekonstruktivistisch diskursanalytischen Intersektionalitätsanalyse. In: Bereswill, Mechthild/ Degenring, Folkert/ Stange, Sabine (Hg.): Intersektionalität und Forschungspraxis. Münster: 172-191.

Tuider, Elisabeth (2013): Migrationsregime und Arbeit. Diskursive Regulierungen der Grenzgebiete Mexikos. In: Giebeler, Cornelia/ Rademacher, Claudia/ Schulze, Erika (Hg.): Intersektionen von race, class, gender, body: Theoretische Zugänge und qualitative Forschungen in Handlungsfeldern der Sozialen Arbeit. Opladen: 225-244.

Vilain, Michael/ Dağlar, Nilgün (2006): "Integration durch Ehrenamt?" Explorative Kurzstudie im Südkreis Borken/ NRW. Unveröffentlichter Abschlussbericht.

Wachter, Thomas (2001): Stiftungen: Zivil- und Steuerrecht in der Praxis. Köln.

Weidner, Stefan (2017): Wir sind nie monokulturell gewesen. In: Leggewie, Claus/ Zifonun, Darius /Lang, Anne-Katrin /Siepmann, Marcel/ Hoppen, Johanna (Hg.): Schlüsselwerke der Kulturwissenschaften. Bielefeld: 17-20.

Weingarten, Elmar/ Sack, Fritz (1979): Ethnomethodologie. Die methodische Konstruktion der Realität. In: Weingarten, Elmar/ Sack, Fritz/ Schenkein, Jim (Hg.): Ethnomethodologie. Beiträge zu einer Soziologie des Alltagshandelns. Frankfurt am Main: 7-28.

Weischer, Christoph (2011): Sozialstrukturanalyse. Grundlagen und Modelle. Wiesbaden.

Weiß, Anja/ Koppetsch, Cornelia u.a. (Hg.) (2001): Klasse und Klassifikation. Die symbolische Dimension sozialer Ungleichheit. Wiesbaden.

West, Candance/ Zimmerman, Don H. (1987): Doing Gender. In: Gender and Society, 1/ 2: $125-151$

West, Candance/ Fenstermaker, Sarah (1995): Doing difference. In: Gender \& Society, 9/ 1: 8-37.

Wetterer, Angelika (2004): Widersprüche zwischen Diskurs und Praxis. Gegenstandsbezug und Erkenntnispotenziale einer sozialkonstruktivistischen Perspektive. In: Helduser, Urte u.a.: Under construction? Konstruktivistische Perspektiven in feministischer Theorie und Forschungspraxis. Frankfurt a.M./ New York: 58-67.

Wigand, Klaus/ Stolte, Stefan/ Heuel, Markus/ Haase-Theobald, Cordula (2011): Stiftungen in der Praxis : Recht, Steuern, Beratung. Wiesbaden.

Wilde, Gabriele (2010): Zivilgesellschaftsforschung aus Geschlechterperspektive. Zur Ambivalenz von Begrenzung und Erweiterung eines politischen Handlungsraumes. In: Zimmer, Annette/ Simsa, Rita von (Hg.): Forschung zu Zivilgesellschaft, NPOs und Engagement, Bürgergesellschaft und Demokratie 46. Wiesbaden: 209227.

Wimmer, Andreas (2008): Elementary strategies of ethnic boundary making. In: Ethnic and Racial Studies, 31/6: 1025-1055

Wimmer, Andreas/ Glick-Schiller, Nina (2002): Methodological Nationalism and beyond: nation-state building, migration and social science. In: Global Networks 2, 4: 301-334. 
Winker, Gabriele/ Degele, Nina (2009): Intersektionalität. Zur Analyse sozialer Ungleichheiten. Bielefeld.

Wolf, André Christian (2009): Bürgerstiftungen als Akteure der Stadtentwicklung. Erkundung der Beiträge von Bürgerstiftungen zur Entwicklung von Stadt und Region. Aachen.

Wolf, André Christian/ Dağlar, Nilgün (2007): Kleingärten: Bunter als gedacht. Kleingärten als Orte für Bürgerengagement und Integration. In: Stadt und Grün, Heft 9/2007: 39-42.

Y1ld1z, Erol (2014): Postmigrantische Perspektiven. Aufbruch in eine neue Geschichtlichkeit. In: Y1ldız, Erol/ Hill, Marc (Hg.): Nach der Migration. Postmigrantische Perspektiven jenseits der Parallelgesellschaft. Bielefeld.

Zimmer, Annette (2007): Vereine - Zivilgesellschaft konkret. Wiesbaden.

Zimmer, Annette (2005): Stiftungen als Organisationen der Zivilgesellschaft. In: Strachwitz, Rupert Graf/ Mercker, Florian (Hg.): Stiftungen in Theorie und Praxis. Handbuch für ein modernes Stiftungswesen. Berlin: 9-21.

Zimmer, Annette (2002a): Dritter Sektor und soziales Kapital. Münsteraner Diskussionspapiere zum Nonprofit-Sektor Nummer 19. Münster.

Zimmer, Annette (2002b): Bürgerengagement, Zivilgesellschaft und Dritter Sektor vor Ort - Standortbestimmungen und Entwicklungsperspektiven. In: Breit, Gotthard/ Massing, Peter (Hg.): Bürgergesellschaft. Zivilgesellschaft. Dritter Sektor. Schwalbach: 39-59.

Zimmer, Annette (1996): Vereine, Basiselement der Demokratie : eine Analyse aus der Dritte-Sektor-Perspektive. Opladen.

Zimmer, Annette/ Priller, Eckhard (2007): Gemeinnützige Organisationen im gesellschaftlichen Wandel. Ergebnisse der Dritte Sektor-Forschung. Wiesbaden.

Zimmer, Annette/ Priller, Eckhard (2003): Dritte-Sektor-Organisationen zwischen „Markt“ und „Mission“. In: Grosewinkel, Dieter u.a. (Hg.): Zivilgesellschaft - national und transnational. WZB Jahrbuch. Berlin: 105-128.

Zimmermann, Don H./ Wieder Lawrence D. (1970): Ethnomethodology and the problem of social order. Comment to Denzin. In: Douglas, J.D. (Hg.): Understanding Everyday Life. Chicago: 287-295. 


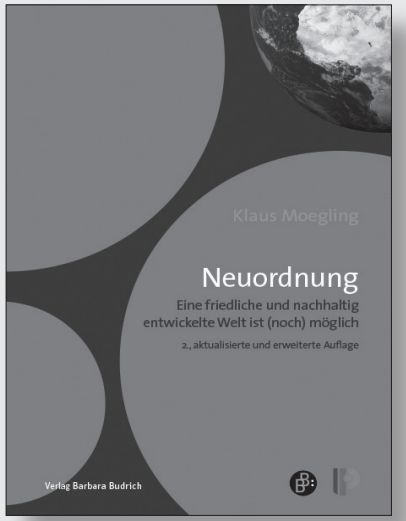

Klaus Moegling

Neuordnung

Eine friedliche und nachhaltig entwickelte Welt ist (noch) möglich - Analyse, Vision und Entwicklungsschritte aus einer holistischen Sicht

2., aktualisierte und erweiterte Auflage 2019

277 Seiten $•$ Kart. $\cdot 24,80 €(D) \cdot 25,50 €(A)$

ISBN 978-3-8474-2344-7 • eISBN 978-3-8474-1480-3

Krieg, soziale Ungleichheit, Klimaerwärmung. Die Welt steht täglich vor neuen Herausforderungen, die, so Klaus Moegling, nur durch eine radikale Neuordnung bewältigt werden können. Seine Botschaft lautet: Eine friedliche und am Prinzip der Nachhaltigkeit orientierte Welt ist möglich. Und: Die Neuordnung kann bereits jetzt mit den ersten notwendigen Schritten beginnen.

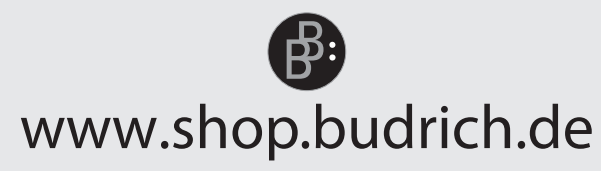




\section{Nilgün Dağlar-Sezer}

\section{Stiftungen, Zivilgesellschaft und soziale Differenz}

Eine qualitative Studie zu gesellschaftspolitischen Stiftungsprojekten

Stiftungen gelten als vielseitige Organisationen der Zivilgesellschaft: Zum einen möchten sie öffentlich wirksam sein, darüber hinaus sind sie aber auch gemeinwohlorientiert. Das qualitativ empirisch angelegte Forschungsprojekt untersucht anhand von Interviews mit Expert*innen, wie Stiftungen im Rahmen ihrer Projektarbeit verschiedene Zielgruppen hervorbringen und soziale Differenzgruppen argumentativ vermengen.

Die Autorin: Nilgün Dağlar-Sezer, M.A., PostDoc im Forschungsprojekt FORTESY im Fachbereich Technik \& Diversity, Universität Paderborn 